Published in final edited form as:

Cochrane Database Syst Rev. ; (3): CD006363. doi:10.1002/14651858.CD006363.pub2.

\title{
Regular treatment with salmeterol for chronic asthma: serious adverse events
}

\author{
Christopher J Cates ${ }^{1}$ and Matthew J Cates ${ }^{2}$ \\ ${ }^{1}$ Population Health Sciences and Education, St George's University of London, London, UK. \\ ${ }^{2}$ Torbay Hospital, Torquay, UK
}

\begin{abstract}
Background-Epidemiological evidence has suggested a link between beta 2 -agonists and increases in asthma mortality. There has been much debate about possible causal links for this association, and whether regular (daily) long-acting beta 2 -agonists are safe.

Objectives-The aim of this review is to assess the risk of fatal and non-fatal serious adverse events in trials that randomised patients with chronic asthma to regular salmeterol versus placebo or regular short-acting beta 2 -agonists.
\end{abstract}

Search methods-We identified trials using the Cochrane Airways Group Specialised Register of trials. We checked websites of clinical trial registers for unpublished trial data and FDA submissions in relation to salmeterol. The date of the most recent search was August 2011.

Selection criteria-We included controlled parallel design clinical trials on patients of any age and severity of asthma if they randomised patients to treatment with regular salmeterol and were of at least 12 weeks' duration. Concomitant use of inhaled corticosteroids was allowed, as long as this was not part of the randomised treatment regimen.

Data collection and analysis-Two authors independently selected trials for inclusion in the review. One author extracted outcome data and the second checked them. We sought unpublished data on mortality and serious adverse events.

Copyright @ 2013 The Cochrane Collaboration. Published by John Wiley \& Sons, Ltd.

Contact address: Christopher J Cates, Population Health Sciences and Education, St George's University of London, Cranmer Terrace, London, SW17 0RE, UK. ccates@sgul.ac.uk. .

Editorial group: Cochrane Airways Group.

Publication status and date: Edited (no change to conclusions), published in Issue 5, 2013.

Review content assessed as up-to-date: 17 August 2011.

CONTRIBUTIONS OF AUTHORS: CJC: Conception of the idea, study selection and data collection, statistical analysis, and cowriting of the review. MJC: Background information (including Appendices), study selection and data collection and co-writing of the review

DECLARATIONS OF INTEREST: None known.

DIFFERENCES BETWEEN PROTOCOL AND REVIEW: Risk difference was not used as the primary metric for analysis of rare events, due to new advice in the latest revision of the Handbook (Higgins 2008). We have added sensitivity analysis to the review to investigate the impact of considering drug-related SAEs, paper publication SAEs, and combining SAEs with all other minor events. The primary analysis has now been carried out using Peto odds ratios in order to avoid the need for continuity corrections for zero cells. 
Main results-The review includes 26 trials comparing salmeterol to placebo and eight trials comparing with salbutamol. These included 62,815 participants with asthma (including 2,599 children). In six trials (2,766 patients), no serious adverse event data could be obtained.

All-cause mortality was higher with regular salmeterol than placebo but the increase was not significant (Peto odds ratio (OR) 1.33 (95\% CI 0.85 to 2.08)). Non-fatal serious adverse events were significantly increased when regular salmeterol was compared with placebo (OR $1.1595 \%$ CI 1.02 to 1.29). One extra serious adverse event occurred over 28 weeks for every 188 people treated with regular salmeterol (95\% CI 95 to 2606). There is insufficient evidence to assess whether the risk in children is higher or lower than in adults. We found no significant increase in fatal or non-fatal serious adverse events when regular salmeterol was compared with regular salbutamol.

We combined individual patient data from the two largest studies (SNS: $\mathrm{n}=25,180$ and SMART: $\mathrm{n}=26,355)$, as all the asthma-related deaths in adults occurred in these studies. In patients who were not taking inhaled corticosteroids, compared to regular salbutamol or placebo, there was a significant increase in risk of asthma-related death with regular salmeterol (Peto OR 6.15 95\% CI 1.73 to 21.84 ). The confidence interval for patients who were taking inhaled corticosteroids is wide and cannot rule in or out an increase in asthma mortality in the presence of an inhaled corticosteroid (Peto OR 2.03 95\% CI 0.82 to 5.00).

Authors' conclusions-In comparison with placebo, we have found an increased risk of serious adverse events with regular salmeterol. There is also a clear increase in risk of asthmarelated mortality in patients not using inhaled corticosteroids in the two large surveillance studies. Although the increase in asthma-related mortality was smaller in patients taking inhaled corticosteroids at baseline, the confidence interval is wide, so we cannot conclude that the inhaled corticosteroids abolish the risks of regular salmeterol. The adverse effects of regular salmeterol in children remain uncertain due to the small number of children studied.

\section{Medical Subject Headings (MeSH)}

Adrenergic beta-Agonists [therapeutic use]; Albuterol [administration \& dosage; adverse effects; "analogs \& derivatives; therapeutic use]; Anti-Asthmatic Agents [administration \& dosage; " adverse effects]; Asthma [drug therapy; ${ }^{*}$ mortality]; Bronchodilator Agents [administration \& dosage; *adverse effects]; Cause of Death; Placebos [therapeutic use]; Randomized Controlled Trials as Topic

\section{MeSH check words}

Adult; Child; Humans

\section{BACKGROUND}

\section{Description of the condition}

There is currently no universally accepted definition of the term "asthma". This is in part due to an overlap of asthmatic symptoms with those of other diseases such as chronic obstructive pulmonary disease (COPD), but is also due to the probable existence of more than one underlying pathophysiological process. There are, for example, wide variations in 
the age of onset, symptoms, triggers, associations with allergic disease and the type of inflammatory cell infiltrate seen in patients diagnosed with severe asthma (Miranda 2004). Patients with all forms and severity of disease will typically have intermittent symptoms of cough, wheeze and/or breathlessness. Underlying these symptoms there is a process of variable, at least partially reversible airway obstruction, airway hyper responsiveness and, in most cases, chronic inflammation.

Airway obstruction-Patients with a history of asthma demonstrate chronic changes within the airways including goblet cell hyperplasia, airway smooth muscle (ASM) hyperplasia and hypertrophy (Ebina 1993;Ordonez 2001; Woodruff 2004) and excess myofibroblasts with increased subepithelial collagen deposition (Brewster 1990). In the acute setting, in patients who have died of status asthmaticus airway obstruction is evident from air-trapping and lung hyperinflation with mucus plugging of the small and large airways (Dunnill 1960; Kuyper 2003). There is also shedding of ciliated bronchial mucosal cells, inflammatory cell infiltrates and submucosal oedema with transudation of fluid into the bronchial lumen (Carroll 1993). It is more difficult to measure the degree of ASM contraction (bronchoconstriction) at post-mortem studies although evidence for a role of bronchoconstriction in airway narrowing comes from other sources.

Airway hyper responsiveness-Patients with asthma typically display a degree of 'airway hyper responsiveness' to inhaled allergens (Cockcroft 2006), and to a variety of chemical stimuli including histamine, serotonin, bradykinin, prostaglandins, methacholine and acetylcholine as well as other triggers such as exercise, deep inhalation and inhalation of cold air (Boushey 1980). Bronchoconstriction is implicated as the primary effector mechanism of airway narrowing in these responses. This is because of both the short time frame of the response and because many of these stimuli typically either cause bronchoconstriction directly in vitro or promote bronchoconstriction through interference with the autonomic control of ASM. Further evidence comes from findings that this response can be abolished or diminished by bronchodilator medications such as atropine and beta $_{2}$-agonists (Phillips 1990; Simonsson 1967); although beta 2 -agonists in particular may have additional mechanisms of action. Whether airway hyper responsiveness relates primarily to an abnormality of ASM, to increased ASM bulk (Wiggs 1990), to aberrant autonomic control or reflex pathways, or to physical damage to the airway epithelium remains to be established. Regular use of salbutamol has, however, been shown to increase airway hyper responsiveness to allergen exposure and produce tolerance to the protective effect of salbutamol against bronchoconstriction induced by both methacholine and allergens (Cockcroft 1993).

Inflammation-It has long been thought that the histological changes described above and the phenomenon of airway hyper responsiveness are due to a combined acute and chronic inflammatory response (Bousquet 2000). Patients with status asthmaticus have increased numbers of inflammatory cells including eosinophils and neutrophils, as well as a variety of pro-inflammatory cytokines and chemokines found in bronchial alveolar lavage (Tonnel 2001). In patients with chronic asthma there is also evidence of increased eosinophil numbers (Bousquet 1990), inflammatory cell adhesion molecules (Vignola 1993) and some 
evidence of an association between the extent of inflammation, disease severity and hyperreactivity. This association has however been questioned on the background of a number of negative results (Brusasco 1998), although it is made difficult to prove by the lack of a consistent marker of a sequential and variable inflammatory response (Haley 1998).

\section{Description of the intervention}

\section{Beta $_{2}$-agonists and mortality: an historical perspective}

Time trend data and case control studies: Adrenaline was successfully used in the symptomatic treatment of asthma as far back as 1903 (Tattersfield 2006). Initially given subcutaneously, the inhaled route was tried in 1929 to reduce adverse effects but these remained a problem and in 1940 details of a new agent isoprenaline (isoproterenol) were published in Germany (Konzett 1940). Although isoprenaline was more selective for betaas opposed to alpha-adrenoreceptors, adverse effects including palpitations were still a major problem, particularly with oral administration (Gay 1949) and it first became available as atomizer spray for use in the UK in 1948 (Pearce 2001).

Prior to the 1940s, mortality rates from asthma in a number of countries were stable and low at less than one asthma death per 100,000 people per year (Pearce 2001; Figure 1). During the 1940s and 50s, there was a slight rise in mortality rates and concerns about a possible link to inhaled adrenaline were raised at an early stage (Benson 1948). However, the rise was small and the cause unclear and sales continued to increase with the introduction of aerosol or metered dose inhalers in the early 1960s. During this decade, there was an epidemic of asthma deaths in at least six countries including England, Wales and New Zealand (Figure 1). In all six countries the epidemics coincided with the licensing of an aerosol called 'Isoprenaline Forte', which contained five times the dose of isoprenaline per administration than the standard preparation (Stolley 1972). In other countries including the Netherlands where isoprenaline forte was introduced late and sales volumes low and in the US, where isoprenaline forte was not licensed, no increase in asthma mortality occurred. This was despite an approximate trebling in per capita alternative bronchodilator sales between 1962 and 1968 in the US (Stolley 1972). A detailed review of the epidemic in England and Wales concluded it was not due to changes in death certification, disease classification or an increase in asthma prevalence, but instead was most likely due to new methods of treatment (Speizer 1968). In England and Wales, mortality rates fell following health warnings about the overuse of inhalers and banning of over the counter sales in 1968. It was around this time that more selective beta 2 -agonists such as terbutaline (Bergman 1969) and salbutamol (albuterol) (Cullum 1969) were being developed.

In the late 1970s, a second epidemic of asthma deaths occurred in New Zealand (Figure 1). It was later shown that this epidemic coincided with the introduction and rising sales of fenoterol, a new short-acting beta 2 -agonist (Crane 1989; Figure 2). A significant association between mortality and fenoterol use was demonstrated in three consecutive case-control studies, the latter studies addressing criticisms of the first (Crane 1989; Grainger 1991;Pearce 1990). Furthermore, the relative risk of asthma death in patients prescribed fenoterol increased markedly when analysis was restricted to subgroups defined by markers 
of severity, including previous hospital admission and use of oral corticosteroids. Following the publication of the first case control study, the fenoterol market share in New Zealand fell from $30 \%$ in 1988 to 3\% in 1991 and by the early 1990s the mortality epidemic appeared to be over (Figure 2). During the gradual decline in mortality in New Zealand from its peak in 1979 , total sales of alternative beta 2 -agonists, including salbutamol, gradually rose and the use of inhaled corticosteroids also increased during the latter half of the 1980s (Pearce 2007).

The introduction of long-acting beta $\mathbf{2}_{2}$-agonists-Given the relatively short-lived action of beta ${ }_{2}$-agonists such as salbutamol, in the late 1980s efforts were made to develop longer-acting compounds. Subsequently, the long-acting beta 2 -agonists (LABAs), salmeterol and formoterol were released by Glaxo-SmithKline (GSK) and Novartis, respectively. Both drugs cause bronchodilation that lasts for more than 12 hours, although formoterol has a faster onset of action (Kemp 1993; Ringdal 1998). Given previous concerns about the safety profile of some of the short acting beta 2 -agonists, salmeterol and formoterol were subject to randomised controlled trials on larger numbers of patients. Using these trials, several Cochrane reviews have addressed the efficacy of LABAs in addition to inhaled corticosteroids (Ni Chroinin 2004; Ni Chroinin 2005), in comparison with placebo (Walters 2007), short-acting beta 2 -agonists (Walters 2002), leukotriene-receptor antagonists (Ducharme 2006), and increased doses of inhaled corticosteroids (Greenstone 2005). The beneficial effects of LABAs on lung function, symptoms, quality of life and exacerbations requiring oral steroids have been demonstrated. However, with some studies demonstrating an associated increase in mortality, concerns about the safety profile of LABAs have heightened and there has been much debate about the potential protective role of inhaled corticosteroids.

\section{How the intervention might work}

We have outlined the pharmacology of beta 2 -agonists in detail in Appendix 1. Since the early epidemics in asthma mortality, a number of potential mechanisms have been proposed to explain a relationship to the use of beta 2 -agonists. We discuss these mechanisms in detail in Appendix 2; they include direct toxicity, tolerance, delay in seeking help and reduction in use of inhaled corticosteroids.

\section{Why it is important to do this review}

We have taken a different approach from Salpeter 2006, in that we have not assumed a class effect of long-acting beta ${ }_{2}$-agonists, but we have considered trials comparing regular salmeterol to placebo or regular salbutamol. We have chosen not to include results from trials on formoterol in this review, as there are known differences in the pharmacology properties of salmeterol and formoterol (Van Noord 1996); however formoterol is the subject of another ongoing review.

In view of the difficulty in ascertaining the causation of deaths and serious adverse events (SAEs), we have considered all-cause fatal and non-fatal SAEs as the main outcomes of this review, with asthma-related and cardiovascular events as secondary outcomes. 


\section{OBJECTIVES}

The aim of this review is to assess the risk of mortality and non-fatal serious adverse events in trials which randomised patients with chronic asthma to salmeterol alone

\section{METHODS}

\section{Criteria for considering studies for this review}

Types of studies-We included randomised controlled trials (RCTs) of parallel design, with or without blinding, in which salmeterol alone was randomly assigned to patients with chronic asthma. We excluded studies on people with acute asthma and exercise-induced bronchospasm.

Types of participants-We included patients with a clinical diagnosis of asthma of any age group, unrestricted by disease severity, previous or current treatment.

Types of interventions-We included trials that randomised participants to receive inhaled salmeterol twice daily for a period of at least 12 weeks, at any dose and delivered by any device (metered dose inhalers (MDIs) with chlorofluorocarbons (CFCs) or hydrofluoroalkane (HFAs), or dry powder inhalers (DPIs). We included studies that used comparison groups with placebo or short-acting beta 2 -agonists, and co-intervention with leukotriene receptor antagonists; inhaled or oral corticosteroids or theophylline was allowed as long as they were not part of the randomised intervention, and were therefore not systematically different between groups. We excluded studies that compared different doses of salmeterol or different delivery devices or propellants (with no placebo arm). We excluded studies in which salmeterol was randomised together with an inhaled steroid (in separate inhalers or a combined inhaler), but these studies were included in a separate review (Cates 2009).

\section{Types of outcome measures}

\section{Primary outcomes:}

1. All-cause mortality

2. All-cause non-fatal SAEs

\section{Secondary outcomes:}

1. Asthma-related mortality

2. Asthma-related non-fatal SAEs

3. Respiratory-related mortality

4. Respiratory-related non-fatal SAEs

5. Cardiovascular-related mortality

6. Cardiovascular-related non-fatal SAEs 
7. Asthma-related non-fatal life-threatening events (intubation or admission to intensive care)

8. Respiratory-related non-fatal life-threatening events (intubation or admission to intensive care)

We did not sub-divide outcomes according to whether the trial investigators considered them to be related to trial medication.

\section{Search methods for identification of studies}

Electronic searches-We identified trials using the Cochrane Airways Group Specialised Register of trials, which is derived from systematic searches of bibliographic databases including the Cochrane Central Register of Controlled Trials (CENTRAL), MEDLINE, EMBASE, CINAHL, AMED, and PsycINFO, and handsearching of respiratory journals and meeting abstracts (see Appendix 3 for details). All records in the Specialised Register coded as 'asthma' were last searched in August 2011 using the following terms: (((beta* and agonist*) and (long-acting or "long acting")) or ((beta* and adrenergic*) and (long-acting or "long acting")) or (bronchodilat* and (long-acting or "long acting")) or (salmeterol or formoterol or eformoterol or advair or symbicort or serevent or sere-tide or oxis)) AND (serious or safety or surveillance or mortality or death or intubat* or adverse or toxicity or complications or tolerability)

Searching other resources-We checked reference lists of all primary studies and review articles for additional references. We checked websites of clinical trial registers for unpublished trial data and FDA submissions in relation to salmeterol.

\section{Data collection and analysis}

Selection of studies-Both authors (CJC, MJC) independently assessed studies identified in the literature searches by examining titles, abstract and keywords fields. We obtained studies that potentially fulfilled the inclusion criteria in full text. We independently assessed these full text trial reports for inclusion. We resolved disagreements by consensus. We kept a record of decisions.

Data extraction and management-We extracted data using a prepared checklist before being entered into RevMan 2011. Data included characteristics of included studies (methods, participants, interventions, outcomes) and results of the included studies. We contacted authors of included studies for unpublished adverse event data, and searched manufacturers' websites for further details of adverse events. We recorded all-cause SAEs (fatal and non-fatal) and in view of the difficulty in deciding whether events were asthma related, we noted details of the cause of death and SAEs where they were available.

Assessment of risk of bias in included studies-Both authors assessed the included studies for bias protection (including sequence generation for randomisation, allocation concealment, blinding of participants and assessors, loss to follow-up, completeness of outcome assessment and other possible bias prevention) as either high, low or unclear risk of bias in line with recommendation in the Cochrane Handbook of Systematic Reviews of 
Interventions (Higgins 2008). We resolved disagreements by consensus. We contacted study authors and sponsors to seek clarification where bias protection was unclear.

Assessment of heterogeneity-We explored heterogeneity on the basis of the subgroup and sensitivity analyses outlined below.

Assessment of reporting biases-We inspected funnel plots to assess publication bias.

Data synthesis-The outcomes of this review were dichotomous, and we recorded the number of participants with each outcome event, by allocated treated group. We planned to conduct the primary analysis, mortality, using risk difference, as many studies did not have any deaths in either arm. However, revisions to the Handbook (Higgins 2008) since the protocol was written advise against this approach. Therefore, the risk differences were only used to estimate the absolute impact of treatment. The Peto odds ratio has advantages when events are rare as no continuity correction for zero cells is required, and although it can perform less well with unbalanced treatment arms and large effect sizes, the updated review has now used Peto odds ratio for all primary analyses, and the Mantel-Haenszel random effects model has been used for sensitivity analysis.

Subgroup analysis and investigation of heterogeneity-We conducted subgroup analyses on the basis of dose of salmeterol (usual dose versus high dose), age (adults versus children), severity of asthma, reported corticosteroid use at baseline, comparator used and ethnic groups. We compared subgroups using tests for interaction (Altman 2003).

\section{RESULTS}

\section{Description of studies}

Results of the search-The initial search in October 2007 found 512 abstracts, which we reduced to 504 after removing duplicates. Of these, we identified 154 abstracts that related to salmeterol alone (three were from references listed in identified trials and review articles, and one was only published on the GSK website). We included 32 studies in the review (42 references) and excluded 118: 71 were less than 12 weeks in duration (including 26 single-dose studies); eight were not RCTs; five were dose comparison studies; five are ongoing studies; four were on exercise-induced bronchospasm; four compared with theophylline; three were propellant studies; three were cross-over in design; and seven were for other reasons. We reached consensus on all the included studies after inspection of the full text from papers and websites; there was initial disagreement on six abstracts, five of which we subsequently included and one excluded. We identified additional references to the included studies and one unpublished study from other reviews (SLGA 3014). A further search in July 2008 generated 48 new references, of which two were relevant to this review (Inoue 2007; Pascoe 2006), but we excluded both as they were short-term crossover studies. We identified no further new studies by the latest searches in August 2009 (79 references) or August 2011 (191 references).

Included studies-There were 32 included studies which randomised 62,630 participants (Adinoff 1998; Boulet 1997; Boyd 1995; Britton 1992;Busse 1998; Chervinsky 1999; 
D’Alonzo 1994; D’Urzo 2001;Kavuru 2000; Kemp 1998a; Kemp 1998b; Lazarus 2001; Lenney 1995a; Lenney 1995b; Lundback 1993; Nathan 1999; Nathan 2006; Pearlman 1992; Pearlman 2004; Rosenthal 1999; Russell 1995; Shapiro 2000; Simons 1997; SLGA 3014; SLMF4002;SMART 2006; SNS 1993; Von Berg 1998; Wenzel 1998; Weinstein 1998; Wolfe 2000).

A total of 46,501 (74\%) people completed treatment, encompassing more than 19,000 patient years studied. There were only seven studies on 2380 (Lenney 1995a; Lenney 1995b;Simons 1997;SLGA 3014; Von Berg 1998; Weinstein 1998) who completed a total of 921 patient years of treatment (this is not allowing for the nine-month extension to the two Lenney studies). The remaining studies were in adults and adolescents with most studies randomising participants of 12 years and older; however many studies included few adolescent patients (for example 6\% of all participants in SNS 1993).

Twenty-six studies (34,781 participants) compared salmeterol to placebo and eight studies (28,532 participants) compared salmeterol to regular salbutamol. Two studies included both a placebo and a salmeterol arm (Pearlman 1992; SLGA 3014). The dose of salbutamol as a comparator varied between trials: four studies used salbutamol $200 \mu \mathrm{g}$ four times daily (Boulet 1997; Pearlman 1992; SLGA 3014; SNS 1993); one study used the same regimen for the first three months, then $200 \mu \mathrm{g}$ twice daily for the remaining nine months (Britton 1992); two studies used $200 \mu \mathrm{g}$ twice daily (Lenney 1995a; Lenney 1995b); and one study used $400 \mu \mathrm{g}$ of salbutamol delivered by diskhaler four times daily for the first three months and then twice daily for the following nine months (Lundback 1993).

The duration of trials ranged from 12 to 52 weeks, but in some of the 52-week studies the data collection was separated into two periods. Four trials separated the first three months from the subsequent nine months of follow-up (Britton 1992; Lenney 1995a; Lenney 1995b; Lundback 1993), whilst Von Berg 1998 separated data from the first and second six-month periods.

The dose of salmeterol used was $50 \mu \mathrm{g}$ twice daily (described as $42 \mu \mathrm{g}$ delivered dose in the studies from the USA) in all studies except two which used $100 \mu \mathrm{g}$ twice daily in participants with more severe asthma on high doses of inhaled steroids at study entry (Boyd 1995; SLMF4002). Three trials used either $25 \mu \mathrm{g}$ or $50 \mu \mathrm{g}$ twice daily in children (Lenney 1995a; Lenney 1995b; SLGA 3014).

Concurrent use of inhaled corticosteroids varied in the included studies from zero to $100 \%$; Table 1 lists the inhaled steroid use by study. Only Rosenthal 1999 and Simons 1997 excluded patients who were using inhaled corticosteroids at baseline. Six trials withdrew inhaled corticosteroids from participants for the duration of the trial (Kavuru 2000; Lazarus 2001; Nathan 1999; Nathan 2006; Pearlman 2004; Shapiro 2000).

\section{Risk of bias in included studies}

All studies were double-blind and unlikely to have been subject to selection bias; however lack of comprehensive reporting of fatal and non-fatal SAEs make selective reporting a threat to the validity of this review (see Figure 3). It should however be noted that the 
missing data are from smaller studies, so $96 \%$ of patients in the trials had some information on SAEs.

Allocation-There was very sparse information in the reports of the studies in journals or on the company website in terms of sequence generation and allocation concealment. Most of these studies were supported by GSK, and correspondence with the company indicated a high level of bias protection in sequence generation and allocation concealment for the studies that they sponsor, so it is unlikely that this area is a major concern.

Blinding-All included studies employed a double-blind design.

Selective reporting - SAEs were not well reported in the journal publications, but were available from the controlled trial register on the GSK website for many of the included studies. The only trial that did not receive financial support from the manufacturers of salmeterol (GSK) was Lazarus 2001; the authors of this trial report have provided data on mortality and hospital admissions.

One study was unpublished (SLMF4002), but event information was available from the controlled trial register on the company website. A second unpublished trial had available data from FDA submission documents (SLGA 3014). The GSK trial register also included reports on the following studies Britton 1992 (SLGT02), D'Urzo 2001 (SLGQ94 [521/180]), Kavuru 2000 (SFCA 3002), Lenney 1995a (SLPT01/SMS40093), Lenney 1995b (SLPT02), Lenney 1995c, Nathan 2006 (SAS3004),Pearlman 2004 (SAS3003), Russell 1995 (SALMP/ AH91/D89),Shapiro 2000 (SFCA 3003), SMART 2006 (SGLA5011), SNS 1993 (SNSD920619), Von Berg 1998 (SLGB3019[SLPT09]), Wolfe 2000 (SLGA3010 \& SLGA3011).

Journal publications tend not to list mortality and all SAEs (often restricting reporting to frequent adverse events or those thought to be related to study medication). We could find no SAE data for six trials (Adinoff 1998; Boulet 1997; Chervinsky 1999; D’Alonzo 1994; Kemp 1998a; Pearlman 1992). These accounted for around 2,766 randomised patients, which represents $4 \%$ of the total randomised population. We have sought further information from GSK in relation to Adinoff 1998, Boulet 1997, Boyd 1995, Busse 1998, Chervinsky 1999, D’ Alonzo 1994, Kemp 1998a, Kemp 1998b, Lenney 1995b (in relation to data from the final nine months), Lundback 1993 (in relation to data from the final nine months), Nathan 1999, Pearlman 1992, Rosenthal 1999, SNS 1993 (in relation to patients with any SAE), Weinstein 1998, and Wenzel 1998.

Although the trial identifiers have been confirmed for these studies, many do not have reports on the manufacturers trial website. At this time it has not been possible to obtain information on SAE numbers with the exception of Lenney 1995b (in relation to SAE data from the final nine months), but further information may be available later and can be included when the review is updated.

Other potential sources of bias-Only the two large surveillance studies SMART 2006 and SNS 1993 used independent outcome assessors. For this reason, the primary outcomes for this review are all-cause mortality and SAEs, as these avoid subjective 
judgements on causation. SAEs thought to be related to the study medication are likely to be subject to bias and have not been included in this review.

\section{Effects of interventions}

See: Summary of findings for the main comparison Regular salmeterol compared to placebo for chronic asthma; Summary of findings 2 Regular salmeterol compared to regular salbutamol for chronic asthma

\section{Primary outcomes}

All-cause mortality: Events were sparse in the trials and the presence or absence of a mortality was not always reported in the paper publications.

Salmeterol versus placebo: Data were available from the controlled trial register on the GSK website for 14 studies comparing salmeterol $(\mathrm{N}=15,271)$ with placebo $(\mathrm{N}=14,983)$; this represents $86 \%$ of the randomised patients for this comparison. Deaths only occurred in two of these trials in adults (D'Urzo 2001; SMART 2006), and overall there were 44 deaths on salmeterol and 33 on placebo. The pooled Peto OR was not statistically significant (1.33, 95\% CI 0.85 to 2.08), Analysis 1.1 (see Figure 4). The confidence interval is almost identical using a Mantel-Haenszel OR (1.33, 95\% CI 0.85 to 2.10), and is the same for fixed-effect and random-effects models. The pooled risk difference has a point estimate of an increase of seven in 10,000 , with a CI from five fewer deaths to 20 more deaths per 10,000 treated with salmeterol. There was no statistical heterogeneity in this outcome.

Salmeterol versus salbutamol: Data were available from seven studies comparing salmeterol $(\mathrm{N}=18,199)$ to salbutamol $(\mathrm{N}=9,416)$, representing $93 \%$ of the randomised patients for this comparison. The larger numbers in the salmeterol arm are due to SNS 1993, in which twice as many patients were randomised to salmeterol in comparison to salbutamol. Three studies in adults (Britton 1992; Lundback 1993; SNS 1993) and one study in children (Lenney 1995a) contributed data to the outcome, although almost all the events came from the SNS study. The results were similar to those from the placebo comparison: 55 deaths occurred in the salmeterol arm compared to 23 in the salbutamol arm but again the difference was not statistically significant (Peto OR 1.22, 95\% CI 0.76 to 1.96; Analysis 1.1; see Figure 4). The Mantel-Haenszel odds ratio was very similar (1.23, $95 \%$ CI 0.75 to 2.02). The pooled risk difference was an increase of seven in 10,000, with a CI from six fewer deaths to 20 more deaths per 10,000 treated with salmeterol. There was some heterogeneity in this outcome $\left(\mathrm{I}^{2}=19 \%\right)$ but sensitivity analysis using a fixed effect risk difference gave a very similar result, with a point estimate of six per 10,000 and a CI from eight fewer deaths to 19 more deaths per 10,000 treated with salmeterol.

Subgroup analyses: No subgroup analyses were possible for all-cause mortality as the data were too sparse, and subgroup characteristics were not reported for this outcome.

SAEs (non-fatal all cause): An illustrative example of the definition of SAEs used in trials by GSK is shown in Appendix 4. Whilst the majority of SAEs result in hospitalisations, the number of patients with SAEs tends to be higher than the number who are admitted to 
hospital (see Analysis 1.22 for comparative data from SMART 2006). We have used information on patients with any SAE for these analyses (with the exception of SNS 1993 where this was not reported, so we have used hospital admissions and life-threatening events).

\section{Salmeterol versus placebo}

Combined data from adults and children: Data were available on non-fatal SAEs from the GSK trial register and FDA submissions for 18 studies comparing salmeterol $(\mathrm{N}=15,895)$ to placebo $(\mathrm{N}=15,634)$; this represents $91 \%$ of the randomised patients for this comparison. The studies were largely on adults, but some data from 1333 children in five trials (Russell 1995; Simons 1997; SLGA 3014; Von Berg 1998; Weinstein 1998) contributed to the analysis. The overall result indicated an increased risk of SAEs with salmeterol (Peto OR $1.15,95 \%$ CI 1.02 to 1.29$)$ with low heterogeneity $\left(\mathrm{I}^{2}=0\right)$, Figure 5. Random-effects Mantel-Haenzel modelling gave a very similar result (OR 1.14, 95\% CI 1.01 to 1.28 ) and the CI was unchanged when Boyd 1995 was excluded in view of the higher dose of salmeterol (100 mcg bd) used in this trial.

Since SAEs were rare in the studies (overall $3.6 \%$ of patients on placebo), the risk difference is small at 0.005 (95\% CI 0.001 to 0.008 ); using the placebo arm of SMART 2006 for reference over a 28 -week period there would need to be 188 patients treated (95\% CI 95 to 2606) for one extra SAE to occur, calculated by Visual Rx using the odds ratio and baseline risk of $3.6 \%$. This is illustrated in Figure 6, which shows that for every thousand patients treated with salmeterol there are an extra five patients who will suffer a SAE, so that in comparison to 40 per thousand in the placebo group this rises to 45 per thousand on salmeterol.

Adults with non-fatal SAEs: When the adult data comparing salmeterol with placebo were considered alone, the results showed a similar increase in risk which is also statistically significant (Peto OR 1.14, 95\% CI 1.01 to 1.28; Figure 7). The result was unchanged using Mantel-Haenszel fixed-effect, but became borderline significant using random-effects (OR $1.13,95 \%$ CI 1.00 to 1.28$)$.

Children with non-fatal SAEs: There were only 1333 children reported for this outcome so, although the direction of effect was the same as for the adults, the CI is wide (Peto OR 1.30, 0.82 to 2.85 ; Figure 8 ). The results in children include both the possibility of a greater risk than in adults, but also the possibility of no difference between salmeterol and placebo.

\section{Salmeterol versus salbutamol}

Combined data from adults and children: In contrast, the results from eight studies comparing salmeterol $(\mathrm{N}=18,481)$ to salbutamol $(\mathrm{N}=9,702)$, representing $98 \%$ of the randomised patients for this comparison, showed a reduction in the risk of SAEs with salmeterol which was not statistically significant (Peto OR 0.96, 95\% CI 0.81 to 1.14). A test for interaction between the results comparing salmeterol with placebo and salbutamol was not significant. As it has not proved possible to obtain missing information in relation to the number of patients with any SAEs in SNS 1993, we used the number of hospitalisations 
or life/threatening events reported in the trial for the main analysis. We adopted this approach because the data collection form allowed more than one classification of events, and in contrast to the other studies in the review, the number of SAEs that did not result in hospitalisation was greater than in the hospitalisation category. We carried out a sensitivity analysis combining all non-fatal SAEs (although this carries a risk that patients may have been more than once, which would lead to an over precise estimate) and this is shown in Analysis 1.5. This approach made very little difference to the pooled result (Peto OR 0.98, $95 \%$ CI 0.86 to 1.11$)$.

Adults with non-fatal SAEs: The results in adults do not differ from the overall result described above (Peto OR 0.94, 95\% CI 0.79 to 1.11 ).

Children with non-fatal SAEs: Again the number of children contributing to this outcome is very small, which makes the uncertainty around the results considerable. As with the previous comparison between salmeterol and placebo, it remains possible that the risk may be higher or lower in children than in adults.

We carried out a sensitivity analysis because separate results are reported from Lenney 1995a and Lenney 1995b for the first three months and the subsequent nine months of the trial. Since patients could have contributed adverse event data from both periods of these trials, we did not consider it safe to combine the results, and full 12-month data were not available. Both pooled results are shown in Figure 8, and there is no significant difference shown in either period. For the first three months the pooled Peto OR was 1.37 (95\% CI 0.71 to 2.64 ) and for the nine-month follow-up the pooled Peto OR was 1.17 (95\% CI 0.71 to 1.94$)$.

All-cause SAEs (fatal and non-fatal combined): When fatal and non-fatal SAEs are considered together the findings are very similar to those for the non-fatal events (Analysis 1.6), with a significant increase in risk with regular salmeterol in comparison with placebo (Peto OR 1.16, 95\% CI 1.03 to 1.30), but not in comparison with regular salbutamol.

\section{Secondary outcomes}

\section{Mortality by cause of death}

Asthma-related mortality: The cause of death was only independently assessed in the two large surveillance studies (SMART 2006; SNS 1993). The results for asthma mortality in these studies are in Analysis 1.7; both studies found a similar increase in asthma mortality, but in the earlier SNS 1993 study this three-fold increase in the risk of asthma death was not statistically significant, whilst in SMART 2006 a significant four-fold increase in the risk of asthma death was found. In absolute terms the size of the risk difference for asthma mortality in SMART 2006 was very similar to that found for mortality of any cause; the point estimate for asthma related death being an increase of eight asthma deaths for every 10,000 patients treated with salmeterol for six months, with a CI from two more deaths to 14 more deaths per 10,000 .

The direction and size of the increase in asthma mortality is consistent between the two studies, and although there are differences in study design and the comparator used, if the 
results of the two studies are combined there is a significant increase in asthma mortality with regular salmeterol, (Peto OR 2.94, 95\% CI 1.41 to 6.14 ).

Inhaled corticosteroid use in relation to asthma mortality: We were able to obtain unpublished data from GSK in relation to asthma mortality and the use of corticosteroids at baseline for each of the asthma-related deaths in SNS 1993. This is shown together with similar published data from SMART 2006 in Analysis 1.8. In the subgroup of patients not taking inhaled corticosteroids at baseline the combined increase in asthma mortality is statistically significant (Peto OR 6.43, 95\% CI 2.13 to 19.42). This indicates a clear increase in risk of asthma death when inhaled corticosteroids were not used when the two studies are considered together.

For the subgroup taking inhaled corticosteroids at baseline, the increase in asthma mortality is smaller and not statistically significant (Peto OR 1.49, 95\% CI 0.54 to 4.11). The test for interaction between inhaled corticosteroid use and asthma related deaths shows a relative OR of 0.23 (95\% CI 0.05 to 1.04) and the CI includes the possibility of a $95 \%$ relative reduction in the risk of asthma death, but also a $4 \%$ relative increase in those on baseline inhaled corticosteroids. Moreover it should be pointed out that the CI for those on inhaled corticosteroids at baseline includes the overall three-fold increase in mortality, so this finding cannot be interpreted as meaning that corticosteroids abolish any increased risk of asthma mortality from regular salmeterol.

Any corticosteroid use in relation to asthma mortality: Analysis 1.9 where the results of SMART 2006 \& SNS 1993 are subgrouped according to any corticosteroid use, shows very similar results to Analysis 1.8. Whilst larger effects are shown in those not taking any form of steroid at baseline, the difference between subgroups is again not significant $\left(\mathrm{Chi}^{2}=1.96\right.$, $\mathrm{df}=1,(\mathrm{P}=0.16))$. As no details were provided in the report of SMART 2006 in relation to the number of patients taking oral corticosteroids at baseline, we assumed that the proportion is very small in comparison to those on inhaled corticosteroids. The information on baseline oral corticosteroid use in those who died has been extracted from Table 5 in the paper publication by Nelson et al.

Cardiovascular-related mortality: This outcome was particularly difficult to assess as it was not a primary outcome for SMART 2006 or SNS 1993, so was not subject to independent data verification. Moreover it is very difficult in practice to know which deaths should be included in this category. The only child who died in all the identified studies was in Lenney 1995a; the death is listed both under asthma attack and circulatory arrest. The pooled odds ratio was not statistically significant and small numbers of deaths and classification problems mean that there is wide uncertainty around the finding, see Analysis 1.10.

Asthma-related SAEs: The reporting of disease-specific SAEs on the GSK controlled trial register does not indicate how many patients suffered from more than one event. This represents a risk of over-counting the number of patients suffering an asthma-related SAE in the large studies, so we only included data for this outcome from studies which show individual events on the controlled-trial web reports. The events in these studies are shown in Analysis 1.11. When salmeterol is compared to placebo the increase in asthma-related 
SAEs is significant using Peto OR 1.59 (95\% CI 1.05 to 2.41 ) but is not statistically significant using Mantel-Haenszel random-effects OR 1.48 (95\% CI 0.97 to 2.27). In comparison with salbutamol the decrease in asthma-related SAEs is not statistically significant (OR $0.99,95 \%$ CI 0.54 to 1.81 ).

SAEs in the cardiovascular system: The reports of SAEs in the cardiovascular system suffer from the same difficulty of possible over counting of patients with events. However, in this case there are almost no data except from SMART 2006 and SNS 1993 so the reported events in these studies have been used and are shown in Analysis 1.12. No significant differences are shown comparing salmeterol to placebo or salbutamol.

Within study subgroup analyses: D'Urzo 2001 reported the number of patients with severe asthma exacerbations (defined as hospitalisation, emergency department visit or use of oral prednisolone) in patients treated in primary care. They subgrouped according to the reported inhaled or oral corticosteroid consumption, and we found significant heterogeneity between subgroups. The direction of effect in the moderate dose subgroup was in favour of salmeterol (Peto OR $0.68,95 \%$ CI 0.42 to 1.08 ). However in participants on oral corticosteroids or an inhaled daily dose of more than $1000 \mu \mathrm{g}$, the direction of effect reversed in favour of placebo (OR 1.75, 95\% CI 0.99 to 3.11; Analysis 1.13). The $\mathrm{Chi}^{2}$ test for differences among the three subgroups returned $\mathrm{P}=0.04$, in keeping with the results of logistic regression analysis reported in the paper $(\mathrm{P}=0.038)$. Very similar results were seen when the patients were stratified according to predicted peak flow (Analysis 1.14).

SMART 2006 reported on numerous post-hoc subgroups in relation to the primary outcome of the study (respiratory related deaths or life threatening events). The main results are shown in Analysis 1.15 to Analysis 1.21. Of these, the only comparison that had a statistically significant test for interaction was the post hoc comparison between African Americans and Causcasians (test for subgroup differences: $\mathrm{Chi}^{2}=5.50, \mathrm{df}=1(\mathrm{P}=0.02)$; Analysis 1.16). Whilst it may be tempting to interpret the individual subgroup results according to their statistical significance (such as for the subgroup with PEF less than 60\% predicted as shown in Analysis 1.20), this approach is not the correct way to test for interaction between PEF and salmeterol (Altman 2003); the test for interaction between subgroups does not indicate a significant difference between those with high and low $\%$ predicted PEF (test for subgroup differences: $\mathrm{Chi}^{2}=1.97, \mathrm{df}=1(\mathrm{P}=0.16)$ ).

\section{DISCUSSION}

\section{Summary of main results}

The difference in the risk of all-cause mortality with regular salmeterol when compared with placebo does not reach statistical significance. Non-fatal SAEs were significantly increased in comparison with placebo. These events were uncommon, such that in the largest study (SMART 2006) 3.6\% of the placebo group suffered a SAE over 28 weeks; in comparison in the salmeterol arm of the trial $4.0 \%$ reported a SAE. One additional SAE was found to occur for every 188 people treated with regular salmeterol over 28 weeks, and the play of chance is compatible with one extra event for every 100 to nearly 3000 given regular salmeterol. This increase is largely derived from the studies in adults, and as the number of children 
studied is small there are insufficient data to ascertain how the effect in children compares to that found in adults.

We found no significant differences for all-cause mortality or non-fatal SAEs in trials comparing regular salmeterol with salbutamol. Disease-specific mortality was not easy to assess, but we found no significant differences in cardiovascular deaths. In contrast there was a consistent increase is asthma-related mortality in both SMART 2006 and SNS 1993. The four-fold increase in SMART 2006 was statistically significant whilst the three-fold increase in SNS 1993 was not. The absolute increase in asthma-related mortality in SMART 2006 was very similar to the size of the absolute increase in all-cause mortality; the increase represents eight extra asthma deaths for every 10,000 patients treated for six months. The combined results of SMART 2006 and SNS 1993 showed a significant in increase asthma mortality. This increase was most apparent in patients who did not take inhaled corticosteroids at baseline, but in patients who took inhaled corticosteroids at baseline the wide confidence interval failed to rule out the possibility of a three fold increase in asthma mortality.

\section{Overall completeness and applicability of evidence}

Although a very large number of adults given salmeterol were included in these studies, the rarity of mortality and SAEs means that there is still considerable uncertainty in relation to the size of the effects being investigated. This is a particular problem in children, where the numbers are smaller. There is insufficient evidence to be sure how the results in adults compare with those in children. It has proved difficult for the sponsor of these studies (GSK) to confirm data for some of the studies we identified, since they do not appear on their webbased trial results register, and no data pertaining to adverse events were available for six of the included studies. The missing data are unlikely to alter the direction of the effects seen in this review, but could alter the point estimates and confidence intervals.

It is interesting to note that if this review had relied upon the information on SAEs published in papers, the results would have looked quite different (see Analysis 1.23). The published information does not indicate any significant increase in SAEs when regular salmeterol is compared to placebo in the 2117 patients included in this small subset of the included studies (Peto OR 1.13, 95\% CI 0.65 to 1.95). If the analysis had been confined to SAEs that were thought to be drug related, the small number of events would not have provided sufficient information to draw any conclusions (Analysis 1.28).

Similar differences can be seen between published (Analysis 1.26) and complete data for adverse events (Analysis 1.25). The published drug related adverse events are shown in Analysis 1.27.

\section{Quality of the evidence}

All of the included studies were double blind, and are likely to have had adequate allocation concealment. However, only the two large surveillance studies had independent assessment of the cause of death. This should not be a threat to all-cause mortality and all-cause SAEs (primary outcomes). In contrast the ascertainment of disease-specific outcomes may have 
been subject to bias, and the data were difficult to use as they were presented as numbers of events rather than number of patients who suffered an event.

Almost all of the studies included in this review were sponsored or supported by GSK, and the lack of published data from some of the studies is of concern.

\section{Agreements and disagreements with other studies or reviews}

The findings of this review are in agreement with the findings of Salpeter 2006, who reviewed the trial evidence in relation to asthma exacerbations leading to hospital admissions, life-threatening events or death. However the Salpeter review combined studies on salmeterol and formoterol, and only considered placebo controlled studies. Our findings also match those presented to the FDA summarising the results of salmeterol trials carried out in the USA (FDA GSK USA Studies). The submission reported: “.....analysis shows similar results for hospitalizations due to asthma for subjects receiving ICS compared with salmeterol plus ICS. For subjects not receiving concomitant ICS, there were a higher number of events in salmeterol recipients compared with placebo." The same submission also found a higher incidence of respiratory related SAEs in the salmeterol group compared to placebo and this is shown alongside our results in Analysis 1.29.

\section{The impact of inhaled corticosteroids on adverse events in relation to long-} acting beta $_{2}$-agonists-We compared data provided by GSK in relation to the inhaled corticosteroid status of those patients who died from asthma in SNS 1993 to the findings of SMART 2006. Both studies showed a similar pattern, in that the main increase in asthmarelated mortality occurred in the subgroup of patients who were not on corticosteroids when they were recruited to the study Figure 12.

It is not possible to draw firm conclusions about the risks of salmeterol when used in conjunction with inhaled corticosteroids (Martinez 2006), as the patients in this review were not randomised to inhaled corticosteroid treatment and the test for interaction between the use of inhaled corticosteroids and salmeterol was not significant. We cannot therefore conclude that inhaled corticosteroids abolish the increased risk of asthma mortality in patients taking salmeterol, nor can we assume that mortality rates might not be even lower if inhaled corticosteroids were taken alone. Moreover, the findings of D'Urzo 2001 do not show consistency in the risk of severe asthma exacerbations with salmeterol and inhaled corticosteroids in primary care; beneficial effects of regular salmeterol with moderate doses of inhaled corticosteroids contrast with increased exacerbations in patients on high doses or maintenance oral corticosteroids. Whilst the interactions between inhaled corticosteroids and beta 2 -agonists are complex, corticosteroids do not appear to prevent the development of tolerance during chronic beta 2 -agonist treatment (Hancox 2006b).

The results of Lazarus 2001 provide support for the fact that salmeterol cannot be used to replace inhaled corticosteroids, because although there were no hospitalisations or deaths in the study, replacing triamcinolone with salmeterol did not maintain asthma control; there was a significant increase in exacerbations on salmeterol or placebo in comparison to triamcinolone. Compliance with inhaled corticosteroids is often poor as demonstrated in Sovani 2008, and there is a risk that patients who are using salmeterol and an inhaled steroid 
in separate inhalers may default on the latter without immediate deterioration in their asthma symptoms.

For patients whose asthma is not well-controlled on moderate doses of inhaled corticosteroids, additional salmeterol can give symptomatic benefit, but this may be at the expense of an increased risk of SAEs and asthma-related mortality, risks which are not clearly abolished by inhaled corticosteroids. Therefore, the risks as well as the benefits of regular salmeterol should be discussed with patients; the drug should be discontinued if no symptomatic benefit is achieved and the manufacturers' advice not to increase the dose of salmeterol during exacerbations should be made clear. Salmeterol should not be used as a substitute for inhaled corticosteroids, and adherence with inhaled steroids should be kept under review if separate inhalers are used. Combining the medications in a single inhaler prevents patients taking salmeterol alone. Observational data from Delea 2008 indicated use of salmeterol and fluticasone in a single inhaler resulted in higher use of inhaled corticosteroids in comparison to patients prescribed two separate inhalers.

\section{AUTHORS' CONCLUSIONS}

\section{Implications for practice}

In comparison with placebo, we have found an increased risk of serious adverse events with regular salmeterol. There is also a clear increase in risk of asthma-related mortality in patients not using inhaled corticosteroids in the two large surveillance studies. Although the increase in asthma-related mortality was smaller in patients taking inhaled corticosteroids at baseline, the CI is wide, so it cannot be concluded that inhaled corticosteroids abolish the risks of regular salmeterol. The adverse effects of regular salmeterol in children remain uncertain due to the small number of children studied.

\section{Implications for research}

Data on SAEs should be more fully reported in medical journals. In view of the increasing use of salmeterol in combination with inhaled corticosteroids, further studies investigating the impact of salmeterol alone on SAEs in adults may not be feasible, but studies using a combination of salmeterol and inhaled steroids should collect and fully report data on fatal and non-fatal SAEs. The evidence base for assessing the risks and benefits of salmeterol in children is currently weak.

\section{Acknowledgments}

\footnotetext{
We thank Toby Lasserson, Susan Hansen and Elizabeth Stovold of the Cochrane Airways Group for their assistance in searching for trials and obtaining the abstracts and full reports, and John White for editing the review. We also thank Steve Yancey and Richard Follows from GSK for their help in obtaining data, and Anne Tattersfield for helpful comments. We also thank the authors of Lazarus 2001 for providing additional information from their study.

CRG Funding Acknowledgement: The National Institute for Health Research (NIHR) is the largest single funder of the Cochrane Airways Group.

Disclaimer: The views and opinions expressed therein are those of the authors and do not necessarily reflect those of the NIHR, NHS or the Department of Health.
}

\section{SOURCES OF SUPPORT}


Internal sources:

- St George's University of London, UK.

External sources:

- NIHR Cochrane Programme Grant, UK.

Funding for time to work on this review

\section{SUMMARY OF FINDINGS FOR THE MAIN COMPARISON}

Regular salmeterol compared to placebo for chronic asthma

Patient or population: patients with chronic asthma

Settings:

Intervention: regular salmeterol

Comparison: placebo 


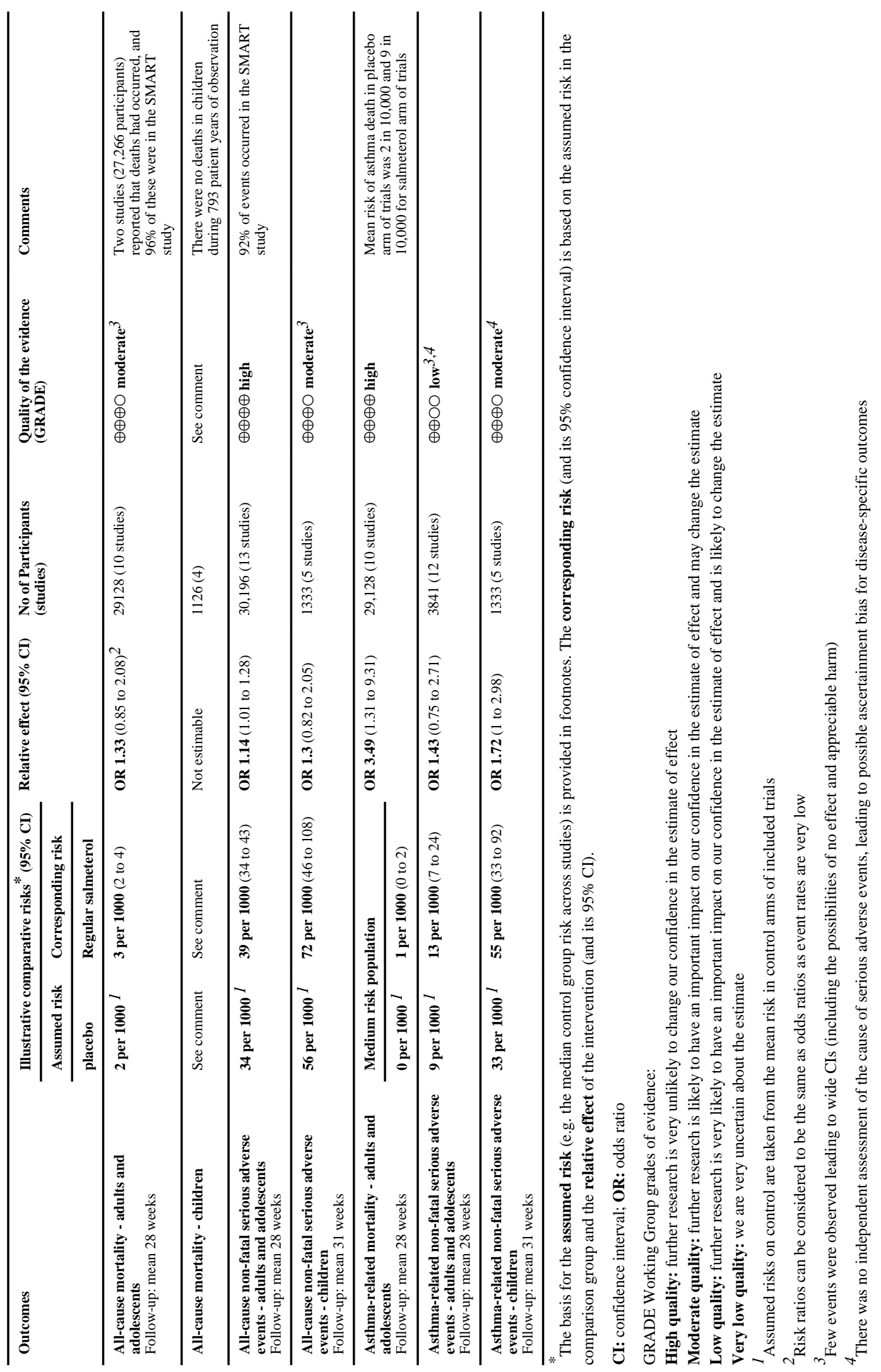




\section{ADDITIONAL SUMMARY OF FINDINGS}

Regular salmeterol compared to regular salbutamol for chronic asthma>

Patient or population: patients with chronic asthma

Settings:

Intervention: regular salmeterol

Comparison: regular salbutamol 


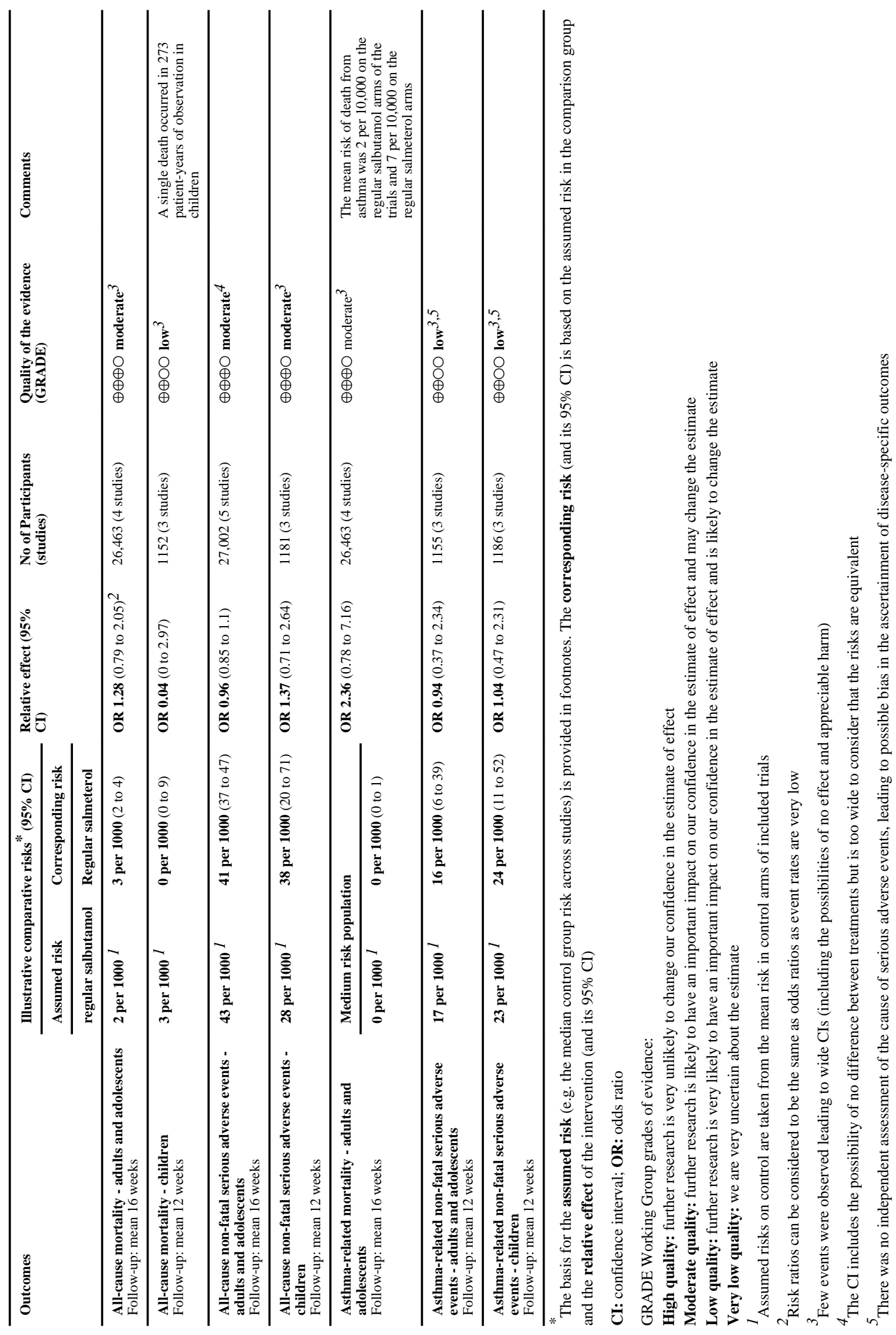

Cochrane Database Syst Rev. Author manuscript; available in PMC 2014 May 09. 


\title{
CHARACTERISTICS OF STUDIES
}

\section{Characteristics of included studies}

\author{
Adinoff 1998
}

\begin{tabular}{|c|c|}
\hline Methods & $\begin{array}{l}\text { Study design: parallel group } 4 \text { groups, multi centre, } 27 \text { in USA (majority primary care) . } 4-6 \text { weeks } \\
\text { initially, } 2 \text { groups weaned from non-steroidal asthma medications, then } 36 \text { weeks treatment } \\
\text { Randomisation: yes, randomised in blocks 5:5:5:1 } \\
\text { Blinding: double blind, double dummy, matching devices } \\
\text { Withdrawals/drop outs: } 75 \text { withdrawals in total, } 61 \text { protocol violations, } 5 \text { lack efficacy } \\
\text { Compliance: not assessed }\end{array}$ \\
\hline Participants & 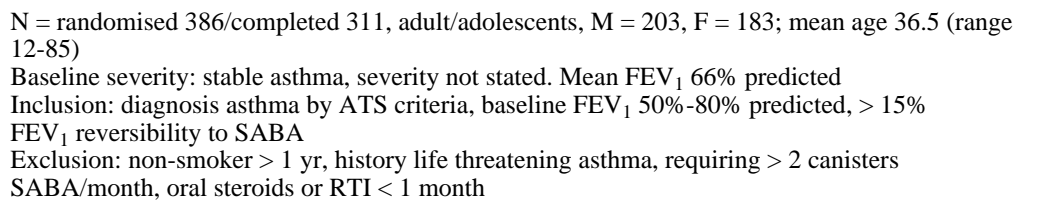 \\
\hline Interventions & $\begin{array}{l}\text { Intervention: salmeterol } 42 \mu \mathrm{g} \text { bd versus (with most patients weaning off nonsteroidal medications } \\
\text { during the course of the study) } \\
\text { Comparison: placebo bd (half the patients randomised to be weaned off nonsteroidal maintenance } \\
\text { treatment) } \\
\text { Device: MDI } \\
\text { Treatment period: } 2-6 \text { weeks weaning, } 36 \text { weeks maintenance } \\
\text { Rescue: albuterol prn } \\
\text { Co-interventions: baseline ICS } 64 \% \text {, cromones } 20 \% \text {, theophylline } 77 \% \text { (only ICS were not weaned } \\
\text { in any group) }\end{array}$ \\
\hline Outcomes & $\begin{array}{l}\text { No website data found for this study. Further information awaited from GSK Paper only reports "no } \\
\text { deaths or serious drug-related adverse events occurred" }\end{array}$ \\
\hline Notes & Funding: grant from GSK \\
\hline \multicolumn{2}{|l|}{ Risk of bias } \\
\hline Bias & Support for judgement \\
\hline $\begin{array}{l}\text { Allocation } \\
\text { concealment } \\
\text { (selection bias) }\end{array}$ & No information \\
\hline $\begin{array}{l}\text { Blinding } \\
\text { (performance } \\
\text { bias and } \\
\text { detection bias) } \\
\text { All outcomes }\end{array}$ & Double-blind, double dummy, matching devices \\
\hline $\begin{array}{l}\text { Selective } \\
\text { reporting } \\
\text { (reporting bias) }\end{array}$ & Not found in GSK trial register \\
\hline
\end{tabular}

Boulet 1997

\begin{tabular}{ll}
\hline Methods & Setting: multicentre study (14 sites in Canada) \\
& Length of intervention period: 12 weeks (2 weeks run-in and 1 week run-out) \\
& Randomisation: no details \\
& Allocation concealment: no details \\
& Design: parallel group \\
& Masking: double-blind \\
& Excluded: 300 enrolled, 228 randomised (all included in the analysis) \\
\hline Participants & 228 adults and adolescents randomised (12 years old or more). \\
& Mean age 38 years. M/F $(\%): 57 / 53 ;$ mean FEV F $_{1} 66 \%$; previous ICS use $73 \%$
\end{tabular}


Inclusion criteria: medical history of mild-to-moderate asthma that required daily pharmacotherapy for at least 6 months. Prebronchodilator $\mathrm{FEV}_{1} 50 \%-80 \%$ with $15 \%$ reversibility following $200 \mu \mathrm{g}$ salbutamol. Symptoms of asthma on $4 / 7$ in last week of run-in period

\begin{tabular}{|c|c|c|}
\hline & \multicolumn{2}{|c|}{$\begin{array}{l}\text { Inclusion criteria: medical history of mild-to-moderate asthma that required daily pharmacotherapy } \\
\text { for at least } 6 \text { months. Prebronchodilator FEV } \mathrm{F}_{1} 50 \%-80 \% \text { with } 15 \% \text { reversibility following } 200 \mu \mathrm{g} \\
\text { salbutamol. Symptoms of asthma on } 4 / 7 \text { in last week of run-in period }\end{array}$} \\
\hline Interventions & \multicolumn{2}{|c|}{$\begin{array}{l}\text { Run-in period: } 2 \text { weeks } \\
\text { LABA: salmeterol } 50 \mu \mathrm{g} \text { bd with placebo inhaler in between } \\
\text { SABA: salbutamol } 200 \mu \mathrm{g} \text { qid } \\
\text { Device: MDI } \\
\text { Treatment period: } 12 \text { weeks } \\
\text { Co-interventions: ICS use } 73 \% \text {; ICS and cromoglycate allowed if enrolled on these for at least one } \\
\text { month and the dose was constant through the study }\end{array}$} \\
\hline Outcomes & \multicolumn{2}{|c|}{$\begin{array}{l}\text { No website data found for this study; further information awaited from GSK } \\
\text { "Adverse events were monitored throughout the study" } \\
\text { The paper reports frequent adverse events but does not report fatal or non-fatal adverse events }\end{array}$} \\
\hline Notes & \multicolumn{2}{|c|}{ Funding: sponsored by GSK } \\
\hline \multicolumn{3}{|l|}{ Risk of bias } \\
\hline Bias & Authors' judgement & Support for judgement \\
\hline $\begin{array}{l}\text { Allocation } \\
\text { concealment } \\
\text { (selection bias) }\end{array}$ & Unclear risk & No information \\
\hline $\begin{array}{l}\text { Blinding } \\
\text { (performance } \\
\text { bias and } \\
\text { detection } \\
\text { bias) } \\
\text { All outcomes }\end{array}$ & Low risk & Double-blind \\
\hline $\begin{array}{l}\text { Selective } \\
\text { reporting } \\
\text { (reporting bias) }\end{array}$ & High risk & Not found in GSK trial register \\
\hline
\end{tabular}

\section{Boyd 1995}

\begin{tabular}{|c|c|c|}
\hline Methods & \multicolumn{2}{|c|}{ Multi-centre parallel group study. Double-blind placebo controlled (use of identical placebo) } \\
\hline Participants & \multicolumn{2}{|c|}{$\begin{array}{l}119 \text { symptomatic asthmatic adults. All patients were on inhaled corticosteroids. Mean age } 47 \text { years. } \\
\text { FEV }_{1} 66 \% \text { predicted at baseline. Most patients took ICS } 1000-2000 \mu \mathrm{g} / \text { day at baseline. At least } 15 \% \\
\text { improvement from baseline in lung function following inhaled salbutamol and at least } 2 \text { acute } \\
\text { asthma exacerbations in the preceding } 18 \text { months were required for inclusion } \\
\text { Exclusion criteria: } \\
\text { - concurrent uncontrolled systemic disease } \\
\text { - having received treatment for an acute respiratory infection in the last } 2 \text { weeks } \\
\text { - or had a FEV } 1<40 \% \text { predicted }\end{array}$} \\
\hline Interventions & \multicolumn{2}{|c|}{12 week duration; salmeterol $100 \mu \mathrm{g}$ twice daily compared with placebo (diskhaler) } \\
\hline Outcomes & \multicolumn{2}{|c|}{$\begin{array}{l}\text { No website data found for this study; further information awaited from GSK } \\
\text { Paper reports seven patients in each treatment group suffered a serious adverse event. Salmeterol } 5 \\
\text { respiratory, one leg injury, one abdominal pain and fever. Placebo } 4 \text { respiratory tract and } 3 \\
\text { hospitalised for unrelated surgical procedures. No mention of fatal events }\end{array}$} \\
\hline Notes & \multicolumn{2}{|c|}{ Funding: sponsored by GSK } \\
\hline \multicolumn{3}{|l|}{ Risk of bias } \\
\hline Bias & Authors' judgement & Support for judgement \\
\hline $\begin{array}{l}\text { Allocation } \\
\text { concealment } \\
\text { (selection bias) }\end{array}$ & Low risk & $\begin{array}{l}\text { Sequence generation by computer generated random } \\
\text { numbers and allocation concealment using } \\
\text { numbered coded inhalers supplied by pharmacy }\end{array}$ \\
\hline $\begin{array}{l}\text { Blinding } \\
\text { (performance } \\
\text { bias and } \\
\text { detection bias) } \\
\text { All outcomes }\end{array}$ & Low risk & $\begin{array}{l}\text { Double-blind placebo controlled (use of identical } \\
\text { placebo) }\end{array}$ \\
\hline
\end{tabular}

Cochrane Database Syst Rev. Author manuscript; available in PMC 2014 May 09. 
Selective Low risk reporting

(reporting bias)
Not found in GSK trial register, but all cause serious adverse events were described by treatment group in the paper

\section{Britton 1992}

\begin{tabular}{|c|c|}
\hline Methods & $\begin{array}{l}\text { A randomised, double-blind, parallel-group study } \\
62 \text { centres in } 13 \text { European countries: Austria (2), Belgium (3),Denmark (6), Finland (4), France (3), } \\
\text { Germany (6), Ireland (5), Italy (5), Netherlands (3), Norway (4), Sweden (9), Switzerland (2), United } \\
\text { Kingdom (10) } \\
\text { Sequence generation: unknown } \\
\text { Allocation concealment: unknown } \\
06 \text { Apr } 1988 \text { to } 19 \text { Oct } 1989\end{array}$ \\
\hline Participants & $\begin{array}{l}\text { Patient characteristics: median age } 49 \text { years (range } 18 \text { to } 81 \text { ). } \mathrm{FEV}_{1} 2.14 \mathrm{~L} .23 \% \text { were in no steroids, } \\
27 \% \text { ICS under } 1000 \mu \mathrm{g} / \text { day, } 36 \% \text { ICS over } 1000 \mu \mathrm{g} / \mathrm{day} \text { and } 14 \% \text { on regular oral steroids } \\
\text { Asthmatic subjects }>18 \text { years of age with either FEV } \mathrm{FV}_{1} \text { or daily PEFR }>50 \% \text { of predicted values, } \\
\text { reversibility in FEV } \mathrm{FE}_{1} .15 \% 15 \text { minutes following the inhalation of salbutamol } 200 \mu \mathrm{g} \text {, and on }>4 \text { of } 7 \\
\text { days in the second week of run-in period } 2 \text { either asthma symptom scores for }>2 \text { or a diurnal } \\
\text { variation in PEFR. } 15 \% \text { were eligible for study participation. Subjects were excluded if they required } \\
\text { a maintenance dose of oral prednisolone }>20 \mathrm{mg} / \text { day, had a clinical or laboratory history of serious } \\
\text { systemic disease, or were treated with beta-receptor antagonists }\end{array}$ \\
\hline Interventions & $\begin{array}{l}\text { Salmeterol } 50 \mu \mathrm{g}(25 \mu \mathrm{g} / \text { actuation }) \text { bd viaMDI }(\mathrm{N}=334) \text {; salbutamol } 200 \mu \mathrm{g} \text { (100 } \mu \mathrm{g} / \text { actuation) QID } \\
\text { via MDI }(\mathrm{N}=333) \\
\text { Co-interventions: } 23 \% \text { were on no steroids, } 27 \% \text { ICS under } 1000 \mu \mathrm{g} / \mathrm{day}, 36 \% \text { ICS over } 1000 \mu \mathrm{g} / \mathrm{day} \\
\text { and } 14 \% \text { on regular oral steroids } \\
\text { Subjects entered } 2 \text { consecutive 1-week run-in periods during which they used a salbutamol inhaler } \\
\text { when necessary for the relief of symptoms. Subjects were then randomised to receive either } \\
\text { salbutamol } 200 \mu \mathrm{g} \text { QID or salmeterol } 50 \mu \mathrm{g} \text { bd, along with a placebo inhaler bd for } 3 \text { months. At the } \\
\text { end of } 3 \text { months, subjects randomised to salmeterol } 50 \mu \mathrm{g} \text { bd continued the same treatment for a } \\
\text { further } 9 \text { months. Subjects randomised to salbutamol had their dosage reduced to } 200 \mu \mathrm{g} \text { bd. All } \\
\text { subjects also received salbutamol inhalers to be used when necessary for the relief of symptoms } \\
\text { during the study }\end{array}$ \\
\hline Outcomes & $\begin{array}{l}\text { Paper reports "Two patients died during the year of the study, one from a stroke caused by a glioma in } \\
\text { the left midbrain after } 10 \text { months of salmeterol and one from septicaemia and renal failure following } \\
\text { an oesophagectomy for an adenocarcinoma after six months treatment with salbutamol" } \\
\text { Website: SLGT02. Data on the initial } 3 \text { month period on-therapy. One fatal adverse event in } \\
\text { salmeterol group (glioma) and one in salbutamol group (adenocarcinoma).Nonfatal serious adverse } \\
\text { events occurred in } 33 \text { subjects in the salmeterol group and } 39 \text { in the salbutamol group }\end{array}$ \\
\hline Notes & Funding: sponsored by GSK \\
\hline \multicolumn{2}{|l|}{ Risk of bias } \\
\hline Bias & Support for judgement \\
\hline $\begin{array}{l}\text { Allocation } \\
\text { concealment } \\
\text { (selection } \\
\text { bias) }\end{array}$ & No information \\
\hline $\begin{array}{l}\text { Blinding } \\
\text { (performance } \\
\text { bias and } \\
\text { detection bias) } \\
\text { All outcomes }\end{array}$ & Double-blind \\
\hline $\begin{array}{l}\text { Selective } \\
\text { reporting } \\
\text { (reporting } \\
\text { bias) }\end{array}$ & Full SAE data on GSK trials register \\
\hline
\end{tabular}

Busse 1998 


\begin{tabular}{|c|c|c|}
\hline Methods & \multicolumn{2}{|c|}{$\begin{array}{l}\text { Study design: parallel group, multicentre (55), USA, } 2 \text { weeks run in/ } 12 \text { weeks treatment } \\
\text { Randomisation: randomised treatment, no method given } \\
\text { Blinding: double-blind, double dummy, matching devices } \\
\text { Withdrawals/drop outs: } 95 \text { withdrawals, } 47 \text { active, } 48 \text { placebo }\end{array}$} \\
\hline Participants & \multicolumn{2}{|c|}{$\begin{array}{l}\mathrm{N}=\text { randomised } 538 \text {, completed } 443 . \mathrm{M}=51 ; \mathrm{F}=68 \text {, adults, mean age } 36 \text { (range } 12-80 \text { ) } \\
\text { Baseline severity: moderate persistent asthma. } \mathrm{FEV}, 62 \% \\
\text { Inclusion: non-smoking. Diagnosis asthma by } \mathrm{ATS} \text { criteria, used SABA daily during } 6 \text { weeks or } \\
\text { more. Baseline } \mathrm{FEV}_{1} 40-80 \% \text { predicted. }>15 \% \mathrm{FEV}_{1} \text { reversibility to SABA. } \\
\text { Symptomatic in run in ASS }>=2 \\
\text { Exclusion: URTI/LRTI }<4 \text { weeks, COPD, CF, unstable asthma, pregnancy, lactation, Use of OS < } 1 \\
\text { month }\end{array}$} \\
\hline Interventions & \multicolumn{2}{|c|}{$\begin{array}{l}\text { Long-acting beta agonist: salmeterol } 42 \mu \mathrm{g} \text { bd } \\
\text { Placebo: bd } \\
\text { Device: MDI } \\
\text { Treatment period: } 12 \text { weeks } \\
\text { Rescue: Salbutamol } 100 \mu \mathrm{g} \text { prn } \\
\text { Co-interventions: ICS } 65 \% \text {, cromones } 7 \% \text {, theophyllines } 30 \% \text { - all fixed doses }\end{array}$} \\
\hline Outcomes & \multicolumn{2}{|c|}{$\begin{array}{l}\text { No website data found for this study. Further information awaited from GSK } \\
\text { Paper reports "Serious adverse events (asthma exacerbation, deep vein thrombosis, respiratory tract } \\
\text { infection) developed in } 2 \text { patients treated with salmeterol; however, these were not considered by the } \\
\text { investigator to be potentially related to treatment." Drug-related adverse events shown in Table } 3\end{array}$} \\
\hline Notes & \multicolumn{2}{|c|}{ Funding: sponsored by GSK } \\
\hline \multicolumn{3}{|l|}{ Risk of bias } \\
\hline Bias & Authors' judgement & Support for judgement \\
\hline $\begin{array}{l}\text { Allocation } \\
\text { concealment } \\
\text { (selection bias) }\end{array}$ & Unclear risk & No information \\
\hline $\begin{array}{l}\text { Blinding } \\
\text { (performance } \\
\text { bias and } \\
\text { detection bias) } \\
\text { All outcomes }\end{array}$ & Low risk & Double-blind, double dummy, matching devices \\
\hline $\begin{array}{l}\text { Selective } \\
\text { reporting } \\
\text { (reporting bias) }\end{array}$ & Low risk & $\begin{array}{l}\text { Not found in GSK trial register, but all-cause } \\
\text { serious adverse events reported in paper }\end{array}$ \\
\hline
\end{tabular}

\section{Chervinsky 1999}

\begin{tabular}{|c|c|}
\hline Methods & $\begin{array}{l}\text { Study design: parallel group, multicentre (18),USA, } 2-4 \text { weeks run in/52weeks treatment and } 2 \text { week } \\
\text { run out. } \\
\text { Randomisation: randomised treatment, using computer generated randomisation schedule } \\
\text { Blinding: double blind, matching devices } \\
\text { Withdrawals/drop outs: } 87 \text { withdrawals, } 45 \text { active, } 42 \text { placebo }\end{array}$ \\
\hline Participants & $\begin{array}{l}\mathrm{N}=\text { randomised } 352 \text {, completed } 265 . \mathrm{M}=179 ; \mathrm{F}=173 \text {, adults, mean age } 30 \text { (range } 12-67 \text { ) } \\
\text { Baseline severity: mild persistent asthma that required pharmacotherapy. } \mathrm{FEV}_{1} 62 \% \text {. } \\
\text { ICS use } 56 \% \text { placebo, } 45 \% \text { salmeterol } \\
\text { Inclusion: non-smoking. Diagnosis asthma by ATS criteria. Baseline } \mathrm{FEV}_{1} 70 \%-90 \% \text { predicted. > } \\
15 \% \mathrm{FEV}_{1} \text { reversibility to SABA. BHR } 20 \% \text { with methacholine } 7.5 \mathrm{mg} / \mathrm{ml} \text { or less. } \\
\text { Exclusion: URTI/LRTI/ < } 6 \text { weeks, abnormal ECG, ongoing passive exposure to tobacco smoke }\end{array}$ \\
\hline Interventions & $\begin{array}{l}\text { Long-actingbeta agonist: salmeterol } 50 \mu \mathrm{g} \text { bd } \\
\text { Placebo: bd } \\
\text { Device: DPI } \\
\text { Treatment period: } 52 \text { weeks } \\
\text { Co-interventions: ICS use } 56 \% \text { placebo, } 45 \% \text { salmeterol. All concomitant treatment } 60 \% \text { and } 47 \% \\
\text { respectively }\end{array}$ \\
\hline
\end{tabular}

Cochrane Database Syst Rev. Author manuscript; available in PMC 2014 May 09. 
Outcomes No website data found for this study. Further information awaited from GSK

Paper reports no deaths in the study. The main focus of this study was on cardiovascular adverse

events, no reporting of all serious adverse events in the paper

\begin{tabular}{lll}
\hline Notes & Funding: sponsored by GSK & \\
\hline Risk of bias & & Support for judgement \\
\hline Bias & Authors' judgement & $\begin{array}{l}\text { Randomised treatment, using computer generated } \\
\text { randomisation schedule }\end{array}$ \\
\hline $\begin{array}{l}\text { Allocation } \\
\text { concealment } \\
\text { (selection bias) }\end{array}$ & Low risk & Double-blind, matching devices \\
\hline $\begin{array}{l}\text { Blinding } \\
\text { (performance } \\
\text { bias and } \\
\text { detection bias) }\end{array}$ & Low risk & \\
$\begin{array}{l}\text { All outcomes } \\
\begin{array}{l}\text { Selective } \\
\text { reporting } \\
\text { (reporting bias) }\end{array}\end{array}$ & High risk & Not found in GSK trial register \\
\hline
\end{tabular}

D’Alonzo 1994

\begin{tabular}{|c|c|}
\hline Methods & $\begin{array}{l}\text { Study design: three treatment parallel group, multicentre (11) study in USA, } 1-2 \text { weeks run in/12 } \\
\text { weeks treatment } \\
\text { Randomisation: yes, but method not given } \\
\text { Blinding: double-blind, double dummy, with } 2 \text { matching inhalers } \\
\text { Withdrawals: } 42 / 322 \text {, by groups - } 15 \text { in salmeterol, } 16 \text { in albuterol, } 11 \text { in placebo } \\
\text { Confounders: differential rates of ICS and cromone use in regular and prn group, use of } \\
\text { theophyllines in run in period }\end{array}$ \\
\hline Participants & $\begin{array}{l}\mathrm{N}=322: \text { albuterol }=108 \text {, placebo }=108 \text {, salmeterol }=106 \\
\text { Age: means - albuterol } 31(14), \text { placebo } 28(12) \text {, salmeterol } 29(12) \\
\text { Severity of asthma: baseline } \mathrm{FEV}_{1} 65 \% \text { predicted } \\
\text { Inclusion: diagnosis asthma by ATS criteria, requiring daily drug treatment for }>6 \text { months. Baseline } \\
\mathrm{FEV}_{1} 50 \%-70 \% \text { predicted, }>15 \% \mathrm{FEV}_{1} \text { reversibility to SABA } \\
\text { Exclusion: smokers }\end{array}$ \\
\hline Interventions & $\begin{array}{l}\text { Long-acting beta agonist: salmeterol } 42 \mu \mathrm{g} \text { bd } \\
\text { Short-acting beta agonist: albuterol } 180 \mu \mathrm{g} \text { qid } \\
\text { Placebo: qid } \\
\text { Device: MDI } \\
\text { Period: } 12 \text { weeks } \\
\text { Rescue: albuterol } 90 \mu \mathrm{g} \text { prn } \\
\text { Co-interventions: } \\
\text { ICS - used by } 20 \% \text { on placebo, } 24 \% \text { on albuterol, } 21 \% \text { on salmeterol } \\
\text { Oral steroids - not at randomisation } \\
\text { Cromones - used by } 9 \% \text { on placebo }, 6 \% \text { on albuterol, } 10 \% \text { on salmeterol } \\
\text { Theophyllines - used only during run in by } 46 \% \text { on placebo, } 50 \% \text { on albuterol, } 43 \% \text { on salmeterol } \\
\text { Ooral beta agonists - not permitted }\end{array}$ \\
\hline Outcomes & $\begin{array}{l}\text { No website data found for this study. Further information awaited from GSK } \\
\text { Paper reports common adverse events thought to be related to treatment, but no serious adverse event } \\
\text { data }\end{array}$ \\
\hline Notes & Funding: sponsored by GSK \\
\hline \multicolumn{2}{|l|}{ Risk of bias } \\
\hline Bias & Support for judgement \\
\hline $\begin{array}{l}\text { Allocation } \\
\text { concealment } \\
\text { (selection bias) }\end{array}$ & No information \\
\hline $\begin{array}{l}\text { Blinding } \\
\text { (performance } \\
\text { bias and } \\
\text { detection bias) }\end{array}$ & $\begin{array}{l}\text { Double-blind, double dummy, with } 2 \text { matching } \\
\text { inhalers }\end{array}$ \\
\hline
\end{tabular}

Cochrane Database Syst Rev. Author manuscript; available in PMC 2014 May 09. 
All outcomes

\begin{tabular}{lll}
\hline $\begin{array}{l}\text { Selective } \\
\text { reporting } \\
\text { (reporting bias) }\end{array}$ & High risk & $\begin{array}{l}\text { Not found in GSK trial register, and no SAE data } \\
\text { in paper }\end{array}$ \\
\hline
\end{tabular}

D’Urzo 2001

\begin{tabular}{|c|c|}
\hline Methods & $\begin{array}{l}\text { A multicenter, randomised, double-blind, parallel-group trial to evaluate the long-term efficacy and } \\
\text { safety of inhaled salmeterol } 50 \mu \mathrm{g} \text { bd compared to short-acting beta }{ }_{2} \text {-agonists as-needed in adult } \\
\text { patients with asthma } \\
15 \text { December } 1994 \text { - } 30 \text { September } 1996\end{array}$ \\
\hline Participants & $\begin{array}{l}\text { Location: } 253 \text { centres in Canada } \\
911 \text { subjects } 18 \text { years of age and older with documented asthma who required regular anti- } \\
\text { inflammatory treatment and used inhaled beta } 2 \text {-agonists to control symptoms more than twice daily } \\
\text { whom the physician believed would benefit from a long-acting beta } 2 \text {-agonist were included in this } \\
\text { study. Females of child-bearing potential could participate if they were using adequate contraceptive } \\
\text { methods, were not pregnant or lactating, and did not plan to become pregnant during the study. } \\
\text { Exclusions included subjects with serious pulmonary or systemic disease, subjects who had received } \\
\text { prior salmeterol, subjects receiving beta-blockers, and subjects with known hypersensitivity to } \\
\text { salmeterol }\end{array}$ \\
\hline Interventions & $\begin{array}{l}24 \text { weeks of one of the following double-blind study treatments: } \\
\text { Salmeterol } 50 \mu \mathrm{g}(2 \text { puffs } 25 \mu \mathrm{g}) \text { via MDI (CFC) bd ( } \mathrm{N}=455) \\
\text { Placebo } 2 \text { puffs via MDI bd }(\mathrm{N}=456) \\
\text { Co-interventions: } 93 \% \text { patients were on ICS and } 5 \% \text { oral steroids ( } 165 \text { under } 500 \mu \mathrm{g} / \text { day, } 535 \text { on } 500 \\
\text { to } 1000 \mu \mathrm{g} / \mathrm{day}, \text { and } 209 \text { on more than } 1000 \mu \mathrm{g} \text { per day or prednisone) }\end{array}$ \\
\hline Outcomes & $\begin{array}{l}\text { Paper publication reports "During the course of the study there were three deaths reported: two in the } \\
\text { salmeterol group (one with congestive heart failure, and one with undetermined cause at autopsy, } \\
\text { although the subject had a history of abdominal pain) and one in the placebo group (myocardial } \\
\text { infarction leading to anoxic encephalopathy). All deaths were judged to be unrelated to study } \\
\text { medication" } \\
\text { Website: SLGQ94 (521/180). Salmeterol group: } 2 \text { fatal SAEs (chronic obstructive lung disease/ } \\
\text { congestive heart failure and abdominal pain). Placebo arm: } 1 \text { fatal SAE (respiratory distress, also } \\
\text { secondary lung edema, myocardial infarction and encephalopathy anoxia). Salmeterol } 24 \text { non-fatal } \\
\text { SAEs, and placebo } 22 \text { non-fatal SAEs }\end{array}$ \\
\hline Notes & Funding: sponsored by GSK \\
\hline \multicolumn{2}{|l|}{ Risk of bias } \\
\hline Bias & Support for judgement \\
\hline $\begin{array}{l}\text { Allocation } \\
\text { concealment } \\
\text { (selection bias) }\end{array}$ & No information \\
\hline $\begin{array}{l}\text { Blinding } \\
\text { (performance } \\
\text { bias and } \\
\text { detection bias) } \\
\text { All outcomes }\end{array}$ & Double-blind \\
\hline $\begin{array}{l}\text { Selective } \\
\text { reporting } \\
\text { (reporting } \\
\text { bias) }\end{array}$ & Full SAE data on GSK trials register \\
\hline
\end{tabular}

FDA GSK USA Studies

\begin{tabular}{ll}
\hline Methods & $\begin{array}{l}\text { This document presents the combined results of GSK studies from the USA in which salmeterol } \\
\text { was compared with placebo in randomised controlled trials. Trials lasted from one to } 52 \text { weeks }\end{array}$ \\
\hline
\end{tabular}


Participants 63 trials involving 17,000 patients with chronic asthma included in Phase II to IV clinical trials in the USA (but results have only been used in this review for patients randomised to salmeterol without ICS)

\begin{tabular}{|c|c|c|}
\hline Interventions & \multicolumn{2}{|c|}{ Salmeterol (with or without additional randomised inhaled corticosteroids) versus placebo } \\
\hline Outcomes & \multicolumn{2}{|c|}{ Respiratory and cardiac serious adverse events } \\
\hline Notes & \multicolumn{2}{|c|}{$\begin{array}{l}\text { Full details are available from http://www.fda.gov/ohrms/dockets/ac/05/briefing/ } \\
\text { 2005-4148B1_01_01-GSK-Serevent.pdf }\end{array}$} \\
\hline \multicolumn{3}{|l|}{ Risk of bias } \\
\hline Bias & Authors' judgement & Support for judgement \\
\hline $\begin{array}{l}\text { Allocation } \\
\text { concealment } \\
\text { (selection bias) }\end{array}$ & Unclear risk & No details of individual studies given \\
\hline $\begin{array}{l}\text { Blinding } \\
\text { (performance bias } \\
\text { and detection } \\
\text { bias) } \\
\text { All outcomes }\end{array}$ & Unclear risk & No details of individual studies given \\
\hline $\begin{array}{l}\text { Selective } \\
\text { reporting } \\
\text { (reporting bias) }\end{array}$ & Unclear risk & No details of individual studies given \\
\hline
\end{tabular}

Kavuru 2000

\begin{tabular}{|c|c|}
\hline Methods & $\begin{array}{l}\text { Setting: multicentre study, USA } \\
\text { Length of intervention period: } 12 \text { weeks } \\
\text { Randomisation: yes (method not stated) } \\
\text { Allocation concealment: not stated } \\
\text { Design: parallel group } \\
\text { Masking: double blind } \\
\text { Excluded: yes } \\
\text { Withdrawals: stated }\end{array}$ \\
\hline Participants & 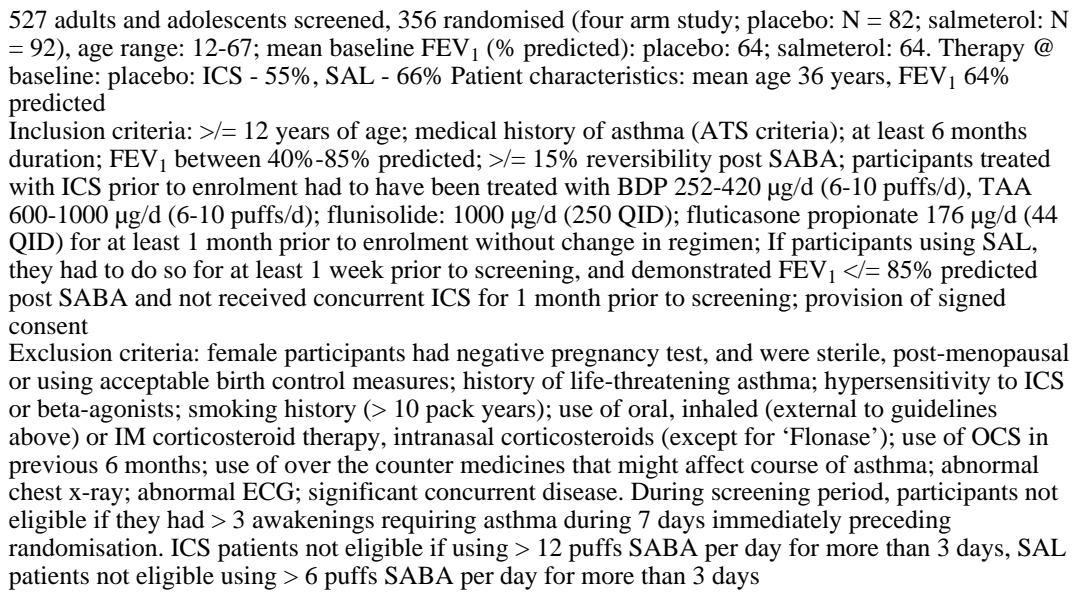 \\
\hline Interventions & $\begin{array}{l}\text { Salmeterol } 50 \mu \mathrm{g} \text { bd versus placebo } \\
\text { Inhaler device: Diskus. Single-blind run-in with a placebo ( } 2 \text { weeks) } \\
\text { Co-interventions: baseline ICS } 26 \% \text {. Group } 1 \text { ( } 250 \text { participants) all had ICS before enrolment, Group } \\
2 \text { ( } 86 \text { participants) had not used ICS for at least a month but had used salmeterol for at least a week. } \\
\text { Protocol suggests that none of the salmeterol or placebo groups continued on ICS as this was a } \\
\text { randomised treatment for the other arms }\end{array}$ \\
\hline Outcomes & $\begin{array}{l}\text { Serious adverse events reported in paper: salmeterol - one asthma exacerbation and one fever. } \\
\text { Placebo - one asthma exacerbation. (None were considered related to study medication by the } \\
\text { investigator.) }\end{array}$ \\
\hline
\end{tabular}

Cochrane Database Syst Rev. Author manuscript; available in PMC 2014 May 09. 
Website SFCA 3002 - SAE data: no fatal SAE. One asthma SAE confirmed in salmeterol and placebo groups. The one patient with fever appears to have also had D \& V, abdominal discomfort, weight loss, dizziness and anaemia

\begin{tabular}{lll}
\hline Notes & Funding: supported by a grant from GSK & \\
\hline Risk of bias & & \\
\hline Bias & Authors' judgement & Support for judgement \\
\hline $\begin{array}{l}\text { Allocation } \\
\text { concealment } \\
\text { (selection } \\
\text { bias) }\end{array}$ & Unclear risk & No information \\
\hline $\begin{array}{l}\text { Blinding } \\
\text { (performance } \\
\text { bias and } \\
\text { detection bias) }\end{array}$ & Low risk & \\
All outcomes & & Double-blind \\
\hline $\begin{array}{l}\text { Selective } \\
\text { reporting } \\
\text { (reporting } \\
\text { bias) }\end{array}$ & Low risk & \\
\hline
\end{tabular}

\section{Kemp 1998a}

\begin{tabular}{|c|c|c|}
\hline Methods & \multicolumn{2}{|c|}{$\begin{array}{l}\text { Study design: two Parallel group, multicentre studies, } 15 \text { centres USA, } 12 \text { weeks. } \\
\text { Randomisation: yes, no method stated } \\
\text { Blinding: double-blind, double dummy, matching inhalers } \\
\text { Withdrawals/drop outs: } 41 \text { described after randomisation } \\
\text { Compliance: }>70 \% \text { reported for all but } 7 \text { subjects }\end{array}$} \\
\hline Participants & \multicolumn{2}{|c|}{$\begin{array}{l}\mathrm{N}=451 \text { randomised ( } 149 \text { salmeterol, } 150 \text { albuterol, } 152 \text { placebo). Adults, } \mathrm{M}=262 \mathrm{~F}=189 \text { mean } \\
\text { age } 31 \text { (SD 14) } \\
\text { Baseline severity: persistent/symptomatic } \mathrm{FEV}_{1} 65 \% \text { predicted; } 43 \% \text { on } \mathrm{ICS} \text { at baseline } \\
\text { Inclusion: diagnosis asthma by ATS criteria. Baseline } \mathrm{FEV}_{1} 50 \%-80 \% \text { predicted, }>15 \% \mathrm{FEV}_{1} \\
\text { reversibility to SABA. Requiring daily drug treatment for }>6 \text { months. } \\
\text { Exclusion: smoking. }\end{array}$} \\
\hline Interventions & \multicolumn{2}{|c|}{$\begin{array}{l}\text { Long-acting beta agonist: salmeterol } 50 \mu \mathrm{g} \text { bd } \\
\text { Short-acting beta agonist: albuterol } 180 \mu \mathrm{g} \text { qid } \\
\text { Placebo: qui } \\
\text { Device: MDI \& dry powder device } \\
\text { Treatment period: } 12 \text { weeks } \\
\text { Rescue: albuterol } 100 \mu \mathrm{g} \text { prn } \\
\text { Co-interventions: ICS } 55 \%-70 \% \text {. Cromones } 7 \%-12 \%\end{array}$} \\
\hline Outcomes & \multicolumn{2}{|c|}{ No website data found for this study. Further information awaited from GSK } \\
\hline Notes & \multicolumn{2}{|c|}{ Funding: sponsored by GSK } \\
\hline \multicolumn{3}{|l|}{ Risk of bias } \\
\hline Bias & Authors' judgement & Support for judgement \\
\hline $\begin{array}{l}\text { Allocation } \\
\text { concealment } \\
\text { (selection bias) }\end{array}$ & Unclear risk & No information \\
\hline $\begin{array}{l}\text { Blinding } \\
\text { (performance } \\
\text { bias and } \\
\text { detection bias) } \\
\text { All outcomes }\end{array}$ & Low risk & Double-blind, double dummy, matching inhalers \\
\hline $\begin{array}{l}\text { Selective } \\
\text { reporting } \\
\text { (reporting bias) }\end{array}$ & High risk & $\begin{array}{l}\text { Not found in GSK trial register, and all cause SAE } \\
\text { data absent from paper }\end{array}$ \\
\hline
\end{tabular}

\section{Kemp 1998b}

Cochrane Database Syst Rev. Author manuscript; available in PMC 2014 May 09. 


\begin{tabular}{|c|c|c|}
\hline Methods & \multicolumn{2}{|c|}{ Design: parallel-group, multicenter (44 centres in USA) } \\
\hline Participants & \multicolumn{2}{|c|}{$\begin{array}{l}506 \text { symptomatic asthmatic adults and adolescents. Mean age } 42 \text { years, baseline } \mathrm{FEV}_{1} 63 \% \text { predicted } \\
\text { Eligibility criteria: average daytime symptom score of } 1 \text { on a } 0 \text { to } 3 \text {-point scale over a } 2 \text {-week } \\
\text { screening period } \\
\text { - use of a short-acting bronchodilator on a daily basis } \\
\text { - FEV } 1 \text { of } 40 \% \text { to } 80 \% \text { predicted } \\
\text { - >= } 15 \% \text { improvement from baseline in } F E V_{1} \text { following inhaled albuterol } \\
\text { - use of one of the following inhaled corticosteroids on a daily basis at a fixed dose that is within } \\
\text { package insert guidelines for a minimum of } 6 \text { weeks prior to the screening visit: beclomethasone } \\
\text { ( } 300-900 \mu \mathrm{g} / \text { day), flunisolide }(1000-2000 \mu \mathrm{g} / \text { day), triamcinolone }(600-1600 \mu \mathrm{g} / \mathrm{day}) \\
\text { Exclusion criteria: concurrent tobacco use } \\
\text { - oral corticosteroid therapy - immunotherapy requiring dosage change } \\
\text { - inability to withdraw asthma/allergy medication before PFTs at screening or clinic visits throughout } \\
\text { the study } \\
\text { - cystic fibrosis, COPD, any significant uncontrolled disease state other than asthma } \\
\text { - any other significant illness } \\
\text { - pregnancy or lactation - contraindication to study medications } \\
\text { - unstable asthma requiring albuterol >= } 12 \text { puffs/day or } 12 \text { puffs for }>3 \text { days/week } \\
\text { - hospitalisation for asthma within } 3 \text { months - mechanical ventilation during an asthma exacerbation } \\
\text { within } 2 \text { years or }>2 \text { albuterol (or equivalent) inhalers/month within } 3 \text { months of screening }\end{array}$} \\
\hline Interventions & \multicolumn{2}{|c|}{12 weeks of treatment with salmeterol $50 \mu \mathrm{g}$ twice daily or placebo. Usual ICS continued } \\
\hline Outcomes & \multicolumn{2}{|c|}{$\begin{array}{l}\text { No website data found for this study. Further information awaited from GSK } \\
\text { Paper reports that "One salmeterol treated patient experienced a serious adverse event (respiratory } \\
\text { failure) that the investigators judged to be possibly due to the study drug." } \\
\text { No report of fatal events }\end{array}$} \\
\hline Notes & \multicolumn{2}{|c|}{ Funding: sponsored by GSK } \\
\hline \multicolumn{3}{|l|}{ Risk of bias } \\
\hline Bias & Authors' judgement & Support for judgement \\
\hline $\begin{array}{l}\text { Allocation } \\
\text { concealment } \\
\text { (selection bias) }\end{array}$ & Low risk & $\begin{array}{l}\text { Randomised by computer generated sequence, } \\
\text { (assignment by opaque consecutive numbered } \\
\text { envelopes) }\end{array}$ \\
\hline $\begin{array}{l}\text { Blinding } \\
\text { (performance } \\
\text { bias and } \\
\text { detection bias) } \\
\text { All outcomes }\end{array}$ & Low risk & Double-blind, identical placebo \\
\hline $\begin{array}{l}\text { Selective } \\
\text { reporting } \\
\text { (reporting bias) }\end{array}$ & High risk & $\begin{array}{l}\text { Not found in GSK trial register, and all cause SAE } \\
\text { data absent from paper }\end{array}$ \\
\hline
\end{tabular}

Lazarus 2001

\begin{tabular}{ll}
\hline Methods & Setting: multicentre study (6 sites in USA). Feb 1997 to Jan 1999 \\
& Length of intervention period: 16 weeks (6 weeks run-in and run-out) \\
& Randomisation: stratified online randomisation \\
& Allocation concealment: random block size. Remote allocation \\
& Design: parallel group \\
& Masking: triple blind \\
& Excluded: 422 enrolled. 361 completed run-in. 339 eligible for run in. 175 patients who were not \\
& well controlled during run in were entered into the SLIC study (Lemanske 2001). 164 randomised in \\
& this trial \\
& Withdrawals: 12 withdrawn in the salmeterol and placebo arms during the randomised treatment \\
& period (placebo 5 withdrew consent. Salmeterol 5 withdrew consent, 1 adverse event. TAA 1 lost to \\
& follow-up) \\
\hline Participants & 164 adults and adolescents randomised. Total of 16 participants aged less than 18 years old. Mean \\
& age 31 years \\
M/F $(\%): 35 / 65 ;$ Caucasian: $70 \%$; mean FEV $193 \%$; previous ICS use 53\% \\
Inclusion criteria: ATS definition of asthma who met recommended treatment criteria for an ICS \\
\hline
\end{tabular}

Cochrane Database Syst Rev. Author manuscript; available in PMC 2014 May 09. 


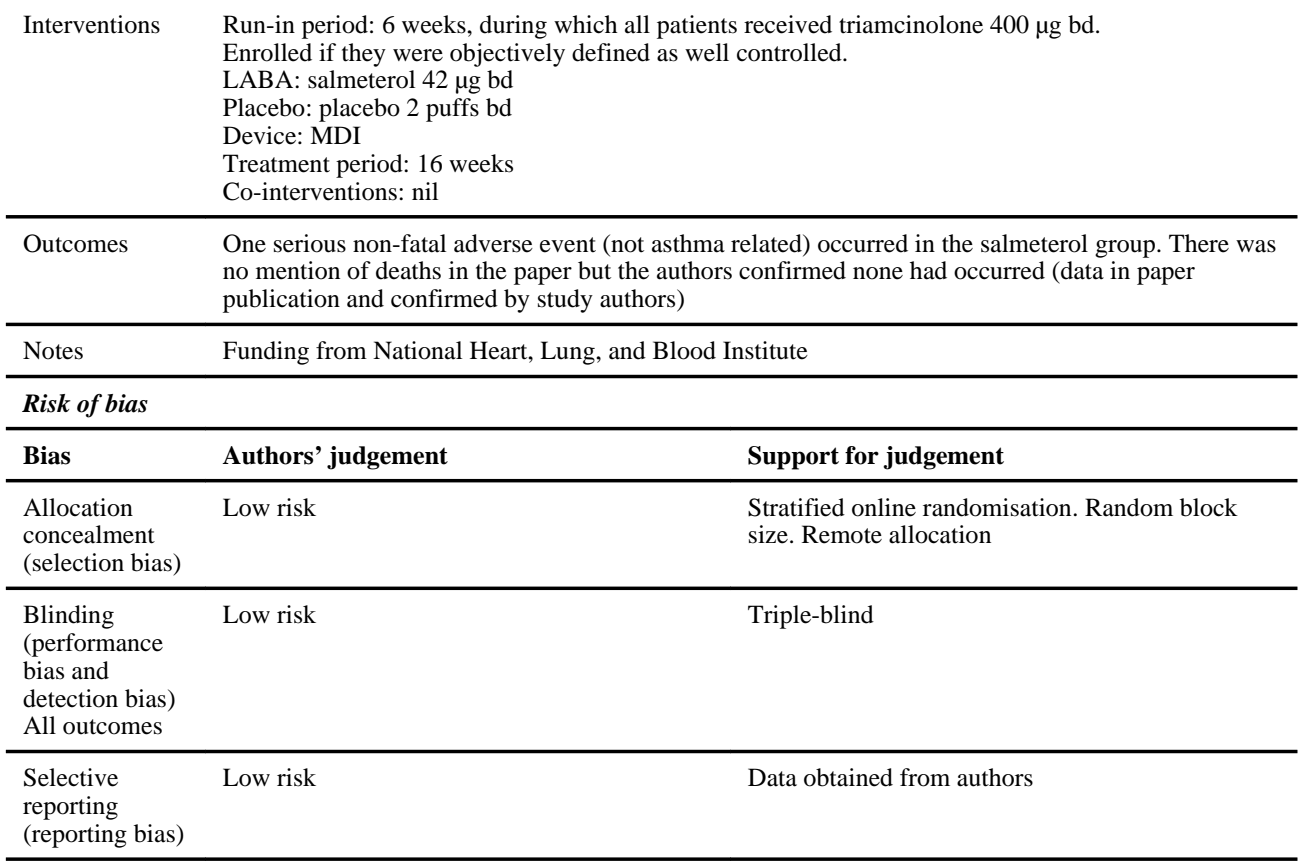

Lenney 1995a

\begin{tabular}{|c|c|}
\hline Methods & $\begin{array}{l}\text { This was a multi-centre, multi-national, double-blind, randomised, 3-limbed, parallel-group study } \\
\text { This study was divided into a 3-month safety and efficacy period followed by a 9-month safety only } \\
\text { period. This is the report of the 3-month efficacy and safety study } \\
\text { Study Period: } 01 \text { Aug } 1990 \text { to } 28 \text { Mar } 1991\end{array}$ \\
\hline Participants & $\begin{array}{l}\text { This study was conducted in } 42 \text { centres in } 9 \text { countries: Germany (4); Ireland (1); Israel (6); Italy (5); } \\
\text { Portugal (2); South Africa (5); Spain (2); Switzerland (1); United Kingdom (16) } \\
\text { Subjects aged } 4 \text { to } 16 \text { years with a history of RAO, who demonstrated at least } 15 \% \text { reversibility of } \\
\text { either PEFR or FEV } 15 \text { minutes following a standard dose of salbutamol ( } 200 \mathrm{ig} \text { ), and who required } \\
\text { regular or, as required, inhaled beta } \text {-agonist treatment for control of their asthma symptoms prior to }_{\text {;he start of study. Subjects who required a change in asthma medication, or who had had an acute }} \\
\text { the sther respiratory tract infection or a hospital admission for their airways disease in the four weeks } \\
\text { preceding the study and subjects who required a maintenance dose of oral steroids were excluded } \\
\text { from the study }\end{array}$ \\
\hline Interventions & $\begin{array}{l}\text { The objectives of the study were to compare the efficacy and safety of inhaled salmeterol } 25 \mu \mathrm{g} \text { and } \\
50 \mu \mathrm{g} \text { bd with salbutamol } 200 \mu \mathrm{g} \text { bd delivered from pressurised inhalers over a } 3 \text {-month period } \\
\text { Subjects were randomly assigned to receive } 1 \text { of the following } 3 \text { treatments via pressurised inhalers: } \\
\text { salmeterol } 25 \mu \mathrm{g} \text { bd }(\mathrm{N}=122) \text {, salmeterol } 50 \mu \mathrm{g} \text { bd }(\mathrm{N}=130) \text {, or salbutamol } 200 \mu \mathrm{g} \text { bd }(\mathrm{N}=122) \text {. } \\
\text { Prior to randomisation subjects completed a } 2 \text {-week run-in period where they took salbutamol, as } \\
\text { needed (prn). } \\
\text { Co-interventions: } 58 \% \text { were on ICS at baseline }\end{array}$ \\
\hline Outcomes & $\begin{array}{l}\text { Paper makes no mention of serious adverse events or mortality } \\
\text { Website: SLPT01 (first } 3 \text { months). No fatal SAEs. Non-fatal SAEs } 5 \text { with salmeterol } 25 \mu \mathrm{g} \text {, } 9 \text { with } \\
\text { salmeterol } 50 \mu \mathrm{g}, 8 \text { with salbutamol } 200 \mu \mathrm{g} \text {. SMS } 40093 \text { provides data on the } 9 \text {-month follow-up } \\
\text { period in which there was one patient on salbutamol who died of an asthma attack with circulatory } \\
\text { arrest }\end{array}$ \\
\hline Notes & Funding: sponsored by GSK \\
\hline \multicolumn{2}{|l|}{ Risk of bias } \\
\hline Bias & Support for judgement \\
\hline $\begin{array}{l}\text { Allocation } \\
\text { concealment }\end{array}$ & No information \\
\hline
\end{tabular}




\begin{tabular}{|c|c|c|}
\hline $\begin{array}{l}\text { Blinding } \\
\text { (performance } \\
\text { bias and } \\
\text { detection bias) } \\
\text { All outcomes }\end{array}$ & Low risk & Double-blind \\
\hline $\begin{array}{l}\text { Selective } \\
\text { reporting } \\
\text { (reporting } \\
\text { bias) }\end{array}$ & Low risk & Full SAE data on GSK trials register \\
\hline
\end{tabular}

\section{Lenney 1995b}

\begin{tabular}{|c|c|}
\hline Methods & $\begin{array}{l}\text { This was a multicenter, multinational, double-blind, randomised, 3-limbed parallel-group study } \\
\text { This study was divided into a 3-month safety and efficacy period followed by a 9-month safety only } \\
\text { period. This is the report of the 3-month efficacy and safety study } 12 \text { Sep } 1990 \text { to } 10 \text { Apr } 1991\end{array}$ \\
\hline Participants & $\begin{array}{l}\text { This study was conducted in } 54 \text { centres in } 13 \text { countries: Austria (3); Belgium (8); Denmark (6); } \\
\text { Finland (2); France (5); Germany (2); Ireland (1); Italy (3); Netherlands (4); Norway (4); Sweden } \\
\text { (11); Switzerland (1); United Kingdom (4) } \\
\text { Subjects aged } 4 \text { to } 16 \text { years of age with a history of RAO were recruited into the study. } \\
\text { Subjects demonstrated at least } 15 \% \text { reversibility of either PEFR or FEV } 15 \text { minutes following a } \\
\text { standard dose of salbutamol ( } 200 \mu \mathrm{g} \text { ). Subjects required regular or, as required, inhaled beta }{ }_{2} \text {-agonist } \\
\text { treatment for control of their asthma symptoms prior to the start of study. Subjects had an asthma } \\
\text { symptom score of } 2 \text { or more on at least five days/nights of the run-in or had diurnal variation in PEFR } \\
\text { of }>15 \% \text { on at least } 7 \text { days of the run-in or had required symptomatic bronchodilator use on at least } 7 \\
\text { days in the run-in period Subjects were excluded if they had required a change in asthma medication } \\
\text { or had been admitted to hospital for their airways disease or had experienced an acute lower } \\
\text { respiratory tract infection in the } 4 \text { weeks preceding the study. Also excluded were patients requiring } \\
\text { maintenance oral steroids or beta-adrenergic antagonists } \\
\text { Mean age } 10 \text { years }\end{array}$ \\
\hline Interventions & $\begin{array}{l}\text { Subjects were randomly assigned to receive } 1 \text { of the following } 3 \text { treatments: salmeterol } 25 \mu \mathrm{g} \text { bd }(\mathrm{N}= \\
\text { 157), salmeterol } 50 \mu \mathrm{g} \text { bd }(\mathrm{N}=160) \text {, or salbutamol } 200 \mu \mathrm{g} \text { bd }(\mathrm{N}=156) \text { via the Diskhaler@ dry } \\
\text { powder inhaler } \\
\text { Co-interventions: } 58 \% \text { were on ICS at baseline }\end{array}$ \\
\hline Outcomes & $\begin{array}{l}\text { Paper makes no mention of serious adverse events or mortality } \\
\text { Website: SLPT02. No fatal SAE. Subjects with non-fatal SAE: } 4 \text { salmeterol } 25 \mu \mathrm{g}, 6 \text { salmeterol } 50 \\
\mu \mathrm{g}, 2 \text { salbutamol } 200 \mu \mathrm{gg} \text {. The number withdrawn due to adverse events were } 5,2 \text {, and } 1 \text { respectively } \\
\text { 9-month data provided for these patients by GSK }\end{array}$ \\
\hline Notes & Funding: sponsored by GSK \\
\hline \multicolumn{2}{|l|}{ Risk of bias } \\
\hline Bias & Support for judgement \\
\hline $\begin{array}{l}\text { Allocation } \\
\text { concealment } \\
\text { (selection } \\
\text { bias) }\end{array}$ & No information \\
\hline $\begin{array}{l}\text { Blinding } \\
\text { (performance } \\
\text { bias and } \\
\text { detection bias) } \\
\text { All outcomes }\end{array}$ & Double-blind \\
\hline $\begin{array}{l}\text { Selective } \\
\text { reporting } \\
\text { (reporting } \\
\text { bias) }\end{array}$ & Full SAE data on GSK trials register \\
\hline
\end{tabular}

Lundback 1993

Cochrane Database Syst Rev. Author manuscript; available in PMC 2014 May 09. 


\begin{tabular}{|c|c|c|}
\hline Methods & \multicolumn{2}{|c|}{$\begin{array}{l}\text { A randomised, double-blind, parallel-group, multi-centre study } \\
\text { Double-blind double-dummy design }\end{array}$} \\
\hline Participants & \multicolumn{2}{|c|}{$\begin{array}{l}\text { Location: } 42 \text { centres in } 11 \text { European countries: Austria; Belgium; Denmark; Finland; France; } \\
\text { Germany; Italy; Portugal; Sweden; Switzerland; United Kingdom } \\
\text { Subjects aged } .18 \text { years with mild to moderate RAO defined by FEV } \text { F }_{1} \text { or mean PEFR of } .50 \% \text { of } \\
\text { predicted values, } .15 \% \text { reversibility of FEV } \text { Ffter salbutamol, and either a total asthma symptom }_{1} \text { after } \\
\text { score } .2 \text { or diurnal variation in PEFR . } 15 \% \text { on four of the seven days prior to randomisation. Key } \\
\text { exclusion criteria were: treatment with maintenance prednisolone }>20 \mathrm{mg} \text { daily; lower respiratory } \\
\text { tract infection, hospitalisation for any aspect of reversible airways disease, course of prednisolone > } \\
\text { 20mg daily within previous } 28 \text { days; pregnancy or lactation, treatment with beta-adrenoreceptor } \\
\text { antagonists; hypersensitivity to beta-adrenoreceptor agonists }\end{array}$} \\
\hline Interventions & \multicolumn{2}{|c|}{$\begin{array}{l}\text { Salmeterol } 50 \mu \mathrm{g} \text { Rotadisk } \mathrm{TM}^{\mathrm{m}} \text { bd }(\mathrm{N}=190) \text {; salbutamol } 400 \mu \mathrm{g} \text { Rotadisk } \mathrm{QID}(\mathrm{N}=198) \text {, and as } \\
\text { needed salbutamol metered-dose inhaler (MDI) } 100 \mu \mathrm{g} / \text { actuation for the first three months, then } \\
\text { salbutamol dropped to } 400 \mu \mathrm{g} \text { bd for the final } 9 \text { months (which remained blinded). Double dummy } \\
\text { used for the initial } 3 \text { months } \\
\text { Inhaled corticosteroids were used by } 57 \% \text { of patients in each group, with another } 7 \% \text { taking oral and } \\
\text { inhaled steroids and } 4 \% \text { taking oral steroids alone }\end{array}$} \\
\hline Outcomes & \multicolumn{2}{|c|}{$\begin{array}{l}\text { Paper reports 12-month follow-up: "Twenty-seven patients ( } 12 \text { receiving salmeterol and } 15 \text { receiving } \\
\text { salbutamol) experienced serious adverse events requiring admission to hospital. Seventeen of these } \\
\text { events involved exacerbations of asthma, of which seven occurred in patients receiving salmeterol and } \\
10 \text { in patients receiving salbutamol. One patient in the salbutamol group died as a result of myocardial } \\
\text { infarction, which was considered unlikely to be related to the study drug." } \\
\text { Website: SLGT06. This is only the first three months of treatment. No fatal SAEs, } 5 \text { subjects with } \\
\text { non-fatal SAEs in salmeterol group ( } 4 \text { with asthma exacerbation and } 1 \text { depressive syndrome). } \\
\text { Salbutamol group } 15 \text { subjects with non-fatal SAE ( } 9 \text { with asthma exacerbation, } 2 \text { cough, } 1 \text { TIA, } 1 \\
\text { fracture femur, } 1 \text { diverticulitis, } 1 \text { surgery) } \\
\text { No GSK website data for the } 9 \text { month follow-up study. } 12 \text {-month data used for this analysis }\end{array}$} \\
\hline Notes & \multicolumn{2}{|c|}{ Funding: sponsored by GSK } \\
\hline \multicolumn{3}{|l|}{ Risk of bias } \\
\hline Bias & Authors' judgement & Support for judgement \\
\hline $\begin{array}{l}\text { Allocation } \\
\text { concealment } \\
\text { (selection } \\
\text { bias) }\end{array}$ & Unclear risk & No information \\
\hline $\begin{array}{l}\text { Blinding } \\
\text { (performance } \\
\text { bias and } \\
\text { detection bias) } \\
\text { All outcomes }\end{array}$ & Low risk & Double-blind, double-dummy design \\
\hline $\begin{array}{l}\text { Selective } \\
\text { reporting } \\
\text { (reporting } \\
\text { bias) }\end{array}$ & Low risk & $\begin{array}{l}\text { 9-month follow-up data missing from GSK website but } \\
\text { SAE data for full } 12 \text { months available from paper }\end{array}$ \\
\hline
\end{tabular}

Nathan 1999

\begin{tabular}{|c|c|}
\hline Methods & Parallel group, multicentre (25) USA, 2 week run-in/26 weeks treatment \\
\hline Participants & $\begin{array}{l}386 \text { adult/adolescents. Mean age } 30 \text {, with a diagnosis of asthma by ATS criteria for at least } 3 / 12 \text {, } \\
\text { baseline } \mathrm{FEV}_{1}: 65 \%-90 \% \text { predicted. }>15 \% \mathrm{FEV}_{1} \text { reversibility } \\
\text { Exclusion: exacerbation asthma, } 1 \text { month, use of ICS/OS }<6 \mathrm{mths} \text {, smoker, requiring }>12 \text { puffs/day } \\
\text { rescue SABA in run in } 4 / 7\end{array}$ \\
\hline Interventions & $\begin{array}{l}\text { Salmeterol } 50 \text { mcg twice daily versus placebo } \\
\text { Device: MDI, no spacer allowed } \\
\text { Treatment period: } 26 \text { weeks } \\
\text { Co-interventions: none permitted (no ICS allowed for the previous } 6 \text { months) }\end{array}$ \\
\hline
\end{tabular}

Outcomes

No website data found for this study. Further information awaited from GSK 
Paper reports one patient in each group experienced a serious adverse event (but all judged unrelated to study drug). Salmeterol one overdose and major depressive disorder, placebo one depression. No published information on any deaths

\begin{tabular}{lll}
\hline Notes & Funding: grant from GSK & \\
\hline Risk of bias & & Support for judgement \\
\hline Bias & Authors' judgement & No details \\
\hline $\begin{array}{l}\text { Allocation } \\
\text { concealment } \\
\text { (selection bias) }\end{array}$ & Unclear risk & \\
\hline $\begin{array}{l}\text { Blinding } \\
\text { (performance } \\
\text { bias and } \\
\text { detection bias) }\end{array}$ & Low risk & Double-blind, double dummy \\
All outcomes & & \\
\hline $\begin{array}{l}\text { Selective } \\
\text { reporting } \\
\text { (reporting bias) }\end{array}$ & Low risk & \\
\hline
\end{tabular}

\section{Nathan 2006}

\begin{tabular}{lll}
\hline Methods & Parallel group multicentre study over 12 weeks \\
\hline Participants & $\begin{array}{l}\text { 365 adults and adolescents randomised. Age range: 12-82 years, mean FEV } 168 \% \text { predicted. } \\
\text { Inclusion criteria: FP440-660 } \mu \text { g/d for at least 3 months prior to study entry; FEV } 140 \%-85 \% ; \\
\text { reversibility }>=15 \%\end{array}$ \\
\hline Interventions & $\begin{array}{l}\text { Combination HFA FP/SAL 110/42 bd (220/84) versus CFC SAL 42 bd (84) versus CFC FP 110 bd } \\
\text { (220) versus HFA placebo. Inhaler devices: MDI. Run-in: 2 weeks This review only includes data } \\
\text { from the salmeterol and placebo arms } \\
\text { Co-interventions: ICS at usual dose was an inclusion criterion, but appears to have been withdrawn } \\
\text { in the salmeterol and placebo arms of the study }\end{array}$ \\
\hline Outcomes & $\begin{array}{l}\text { The paper publication mentions one drug-related SAE (an upper GI bleed from the placebo group) } \\
\text { Website: SAS30004. No fatal SAE. One SAE on salmeterol (viral meningitis) and one on placebo } \\
\text { (upper GI bleed) }\end{array}$ \\
\hline Notes & Funding: sponsored by GSK & \\
\hline Risk of bias & Authors' judgement & Support for judgement \\
\hline Bias & Unclear risk & No information \\
\hline $\begin{array}{l}\text { Allocation } \\
\text { concealment } \\
\text { (selection bias) }\end{array}$ & & Double-blind \\
\hline $\begin{array}{l}\text { Blinding } \\
\text { (performance } \\
\text { bias and } \\
\text { detection bias) } \\
\text { All outcomes }\end{array}$ & Low risk & Full SAE data on GSK trials register \\
\hline $\begin{array}{l}\text { Selective } \\
\text { reporting } \\
\text { (reporting bias) }\end{array}$ & Low risk & \\
\hline
\end{tabular}

\section{Pearlman 1992}

\begin{tabular}{ll}
\hline Methods & Parallel-group (3), multicentre (8) in USA \\
\hline Participants & $\mathrm{N}=$ randomised 234; salmeterol $\mathrm{N}=78$; albuterol (salbutamol) $\mathrm{N}=77$; placebo $\mathrm{N}=79$
\end{tabular}

Cochrane Database Syst Rev. Author manuscript; available in PMC 2014 May 09. 
Patients over 12 years old with asthma (as defined by the American Thoracic Society) and nonsmokers. At least 6 months of daily therapy before the start of the study. $\mathrm{FEV}_{1} 50 \%-80 \%$ predicted values

Long-acting beta agonist: salmeterol $42 \mu \mathrm{g}$ bd

Short acting beta agonist: albuterol (salbutamol) $180 \mu \mathrm{g}$ qid

Placebo: qid

Device: MDI

Period treatment: 12 weeks

Rescue: albuterol $90 \mathrm{mcg}$ prn

Co-interventions: ICS $25 \%$, chromones $10 \%$

\begin{tabular}{|c|c|c|}
\hline Outcomes & $\begin{array}{l}\text { No website data found for } \mathrm{t} \\
\text { No information on serious }\end{array}$ & $\begin{array}{l}\text { Iformation awaited from GSK } \\
\text { rted in the paper }\end{array}$ \\
\hline Notes & Funding: grant from GSK & \\
\hline \multicolumn{3}{|l|}{ Risk of bias } \\
\hline Bias & Authors' judgement & Support for judgement \\
\hline $\begin{array}{l}\text { Allocation } \\
\text { concealment } \\
\text { (selection bias) }\end{array}$ & Unclear risk & No information \\
\hline $\begin{array}{l}\text { Blinding } \\
\text { (performance } \\
\text { bias and } \\
\text { detection bias) } \\
\text { All outcomes }\end{array}$ & Low risk & $\begin{array}{l}\text { Double-blind, placebo controlled using } 2 \text { identical } \\
\text { inhalers }\end{array}$ \\
\hline $\begin{array}{l}\text { Selective } \\
\text { reporting } \\
\text { (reporting bias) }\end{array}$ & High risk & $\begin{array}{l}\text { Not found in GSK trial register, and all-cause SAE } \\
\text { data not in paper }\end{array}$ \\
\hline
\end{tabular}

Pearlman 2004

\begin{tabular}{|c|c|}
\hline Methods & $\begin{array}{l}\text { Setting: Multicentre study, USA } \\
\text { Length of intervention period: } 12 \text { weeks } \\
\text { Randomisation: yes (method not reported) } \\
\text { Allocation concealment: not stated } \\
\text { Design: parallel group } \\
\text { Masking: double blind } \\
\text { Excluded: not stated } \\
\text { Withdrawals: not stated (ITT) }\end{array}$ \\
\hline Participants & $\begin{array}{l}\mathrm{N}=360 \text {. Salmeterol arm } \mathrm{N}=92 \text {, placebo arm } \mathrm{N}=87 \\
\text { Study population: males and females } 12 \text { years of age or older, with a diagnosis of asthma using the } \\
\text { American Thoracic Society definition were screened. All subjects were required to have a } \mathrm{FEV}_{1} \text { of } \\
40 \% \text { to } 85 \% \text { predicted normal and }>15 \% \text { reversibility following } 2 \text { puffs of VENTOLIN at screening. } \\
\text { The study population was stratified according to whether or not subjects were treated with inhaled } \\
\text { corticosteroids or inhaled beta2-agoinsts at screening (salmeterol or short-acting beta2-agonists only). } \\
\text { Subjects treated with inhaled corticosteroids must have been treated for at least } 3 \text { months prior to visit } \\
1 \text { and receiving a daily dose of: } 252-336 \mu \mathrm{g} \text { beclomethasone dipropionate, } 600-800 \mu \mathrm{g} \text { triamcinolone } \\
\text { acetonide, } 1000 \mu \mathrm{g} \text { flunisolide, } 400-600 \mu \mathrm{g} \text { budesonide, } 176 \mu \mathrm{g} \text { fluticasone propionate inhalation } \\
\text { aerosol or } 200 \mu \mathrm{g} \text { FP inhalation powder for at least one month prior to visit } 1 \text { with no change in } \\
\text { regimen. Eligible subjects using only, as-needed, short-acting betaagonist therapy were required to } \\
\text { have received treatment for at least one week prior to visit } 1 \text { and have a } 7 \text {-day total symptom score }> \\
7 \text { for the } 7 \text { days prior to visit } 2 \text {. Eligible subjects using salmeterol at baseline were required to have } \\
\text { received salmeterol and asneeded, short-acting beta } 2 \text {-agonists only for at least one week prior to visit } \\
1 \\
\text { No details on distribution between the groups provided. Participants described as symptomatic. } \\
\text { Baseline medication: prn SABA alone: } 142 ; \text { SAL: } 84 \text {; ICS: } 134 \text { (37\%) }\end{array}$ \\
\hline Interventions & $\begin{array}{l}\text { Salmeterol } 42 \mu \mathrm{g} \text { bd via CFC-MDI versus placebo via HFA-MDI. Inhaler device: MDI. } 2 \text { week run- } \\
\text { in. Duration } 12 \text { weeks } \\
\text { Co-interventions: as ICS is part of the randomised treatment for } 2 \text { arms of this study, we have } \\
\text { assumed that the salmeterol and placebo arms did not take ICS }\end{array}$ \\
\hline Outcomes & $\begin{array}{l}\text { Paper reports no serious drug-related adverse events } \\
\text { Website: SAS3003. No fatal or non-fatal SAE in the salmeterol or placebo group }\end{array}$ \\
\hline
\end{tabular}

Cochrane Database Syst Rev. Author manuscript; available in PMC 2014 May 09. 


\begin{tabular}{lll} 
Notes & Funding: sponsored by GSK & \\
\hline Risk of bias & & Support for judgement \\
\hline Bias & Authors' judgement & No information \\
\hline $\begin{array}{l}\text { Allocation } \\
\text { concealment } \\
\text { (selection bias) }\end{array}$ & Unclear risk & \\
\hline $\begin{array}{l}\text { Blinding } \\
\text { (performance } \\
\text { bias and } \\
\text { detection bias) } \\
\text { All outcomes }\end{array}$ & Low risk & Double blind \\
\hline $\begin{array}{l}\text { Selective } \\
\text { reporting } \\
\text { (reporting } \\
\text { bias) }\end{array}$ & Low risk & \\
\hline
\end{tabular}

\section{Rosenthal 1999}

\begin{tabular}{|c|c|c|}
\hline Methods & \multicolumn{2}{|c|}{2 parallel group studies, multi-centre (31) USA, 2 week run in/24 weeks treatment/4 weeks run out } \\
\hline Participants & \multicolumn{2}{|c|}{$\begin{array}{l}408 \text { adult/adolescents with mean age } 28 \\
\text { Inclusion: clinical diagnosis asthma, baseline } \mathrm{FEV}_{1}>70 \% \text { predicted, }>15 \% \mathrm{FEV}_{1} \text { reversibility to } \\
\text { SABA. BHR PD } 20<512 \mu \mathrm{g} \text { or } 7.5 \mathrm{mg} / \mathrm{mL} \text { methacholine } \\
\text { Exclusion: URTI/LRTI }<6 \text { weeks hospitalisation }<12 \text { weeks serious uncontrolled systemic disease }\end{array}$} \\
\hline Interventions & \multicolumn{2}{|c|}{$\begin{array}{l}\text { Salmeterol } 50 \mu \mathrm{g} \text { twice daily or placebo (given by MDI) } \\
\text { Co-interventions: none permitted (patients were not using inhaled corticosteroids) }\end{array}$} \\
\hline Outcomes & \multicolumn{2}{|c|}{$\begin{array}{l}\text { No website data found for this study. Further information awaited from GSK } \\
\text { The paper publication states that there were seven events requiring hospitalisation in the salmeterol } \\
\text { group (respiratory arrest due to alcohol, tonsillitis, mononucleosis, asthma exacerbation with } \\
\text { bronchitis, knee ligament tear, fractured leg and anaphylaxis due to acne treatment). It is not } \\
\text { reported how many patients suffered a serious adverse event. One patient in the placebo group was } \\
\text { hospitalised for status asthmaticus due to pneumonia }\end{array}$} \\
\hline Notes & \multicolumn{2}{|c|}{ Funding: supported by a research grant from GSK } \\
\hline \multicolumn{3}{|l|}{ Risk of bias } \\
\hline Bias & Authors' judgement & Support for judgement \\
\hline $\begin{array}{l}\text { Allocation } \\
\text { concealment } \\
\text { (selection bias) }\end{array}$ & Unclear risk & No information \\
\hline $\begin{array}{l}\text { Blinding } \\
\text { (performance } \\
\text { bias and } \\
\text { detection bias) } \\
\text { All outcomes }\end{array}$ & Low risk & Double blind, placebo controlled, blinded devices \\
\hline $\begin{array}{l}\text { Selective } \\
\text { reporting } \\
\text { (reporting bias) }\end{array}$ & High risk & $\begin{array}{l}\text { Not found in GSK trial register, and paper does not } \\
\text { report number of patients with SAE }\end{array}$ \\
\hline
\end{tabular}

Russell 1995

\begin{tabular}{ll}
\hline Methods & Multi-centre, double-blind, placebo-controlled, parallel-group study 14 March 1992 to 14 March \\
& 1993 \\
\hline Participants & 78 centres in the United Kingdom
\end{tabular}

Cochrane Database Syst Rev. Author manuscript; available in PMC 2014 May 09. 
Male and female asthmatic children aged 4 to 16 years, inclusive, who had been taking at least 200 $\mu \mathrm{g}$ daily of BDP or equivalent for at least 6 months and who were symptomatic and demonstrated a mean PEF of $90 \%$ or less of predicted normal on at least 4 days of baseline. Subjects excluded were those receiving oral 2-adrenoceptor agonist therapy or maintenance oral corticosteroid therapy or those who had a short course of oral corticosteroids in the two weeks prior to the start of the baseline period. Also excluded were those who had received newly prescribed asthma therapy, or had changed asthma therapy in the two weeks prior to the start of the baseline period

Interventions The study was designed to assess the efficacy and safety of inhaled salmeterol xinafoate (SAL) $50 \mu \mathrm{g}$ bd when added to the existing therapy for moderate to severe asthmatic children Subjects remained on their usual prophylactic therapy of at least the maximum licensed dose of BDP or equivalent during the 2-week baseline period, and their usual beta 2 -adrenoceptor agonist therapy was replaced by commercially available salbutamol $200 \mu \mathrm{g}$ Diskhaler (Ventodisks) to be used as required for symptomatic relief. Subjects were then randomised to receive either salmeterol $50 \mu \mathrm{g}$ bd via the Diskhaler $(\mathrm{N}=99)$ or matching placebo bd $(\mathrm{N}=107)$ in addition to their usual prophylactic therapy for 12 weeks Co-interventions: $100 \%$ ICS as this was an inclusion criterion

\begin{tabular}{lll}
\hline Outcomes & $\begin{array}{l}\text { Paper publication reported serious adverse events in 10 patients on salmeterol and 13 on placebo } \\
\text { Website: SALMP/AH91/D89. No fatal SAEs. Salmeterol 10 subjects had non-fatal SAE, placebo 13 } \\
\text { subjects }\end{array}$ \\
\hline Notes & Funding: sponsored by GSK & \\
\hline Risk of bias & & Support for judgement \\
\hline Bias & Authors' judgement & No information \\
\hline $\begin{array}{l}\text { Allocation } \\
\text { concealment } \\
\text { (selection bias) }\end{array}$ & Unclear risk & Double blind \\
\hline $\begin{array}{l}\text { Blinding } \\
\text { (performance } \\
\text { bias and } \\
\text { detection bias) } \\
\text { All outcomes }\end{array}$ & Low risk & \\
\hline $\begin{array}{l}\text { Selective } \\
\text { reporting } \\
\text { (reporting } \\
\text { bias) }\end{array}$ & Low risk & Full SAE data on GSK trials register \\
\hline
\end{tabular}

Shapiro 2000

\begin{tabular}{|c|c|}
\hline Methods & Setting: multicentre study, USA \\
\hline Participants & $\begin{array}{l}349 \text { adults and adolescents randomised (four treatment arm study; placebo: } 93 \text {; salmeterol: } 88 \text {. Data } \\
\text { from } 13 \text { participants excluded from the analysis due to poor procedure at one site) } \\
\text { Inclusion criteria: }>/=12 \text { years; ATS defined asthma of }>/=6 \text { mo duration requiring } \\
\text { pharmacotherapy for at least } 6 \text { months; FEV } V_{1} \text { between } 40 \% \text { and } 85 \% \text { predicted; }>/=15 \% \text { increase in } \\
\text { FEV } 130 \text { minutes after } 2 \text { puffs of albuterol; use of ICS } 12 \text { weeks prior to the study } \\
\text { Exclusion criteria: females with negative pregnancy tests; life-threatening asthma; hypersensitivity to } \\
\text { sympathomimetic drugs/steroids; smoking within previous year; smoking history of }>10 \text { pack years; } \\
\text { use of oral/injectable steroid therapy within } 1 \text { month of study; use of daily oral steroids within } 6 \\
\text { months prior to the study; use of any prescription or over the counter medication that could have } \\
\text { affected asthma or course of treatment; abnormal CXR; clinically significant abnormal } 12 \text {-lead ECGs } \\
\text { history of concurrent disease }\end{array}$ \\
\hline Interventions & $\begin{array}{l}\text { Salmeterol } 50 \mu \mathrm{g} \text { bd via Diskus inhaler versus placebo. Duration: } 12 \text { weeks Co-interventions: } \\
\text { baseline } 100 \% \text { ICS (as this was an inclusion criterion). However the ICS appears to have been } \\
\text { withdrawn from the salmeterol and placebo arms that we considered }\end{array}$ \\
\hline Outcomes & $\begin{array}{l}49 \% \text { completed study in salmeterol arm and } 28 \% \text { in placebo arm } \\
\text { Paper reports "no serious drug-related adverse events. Two patients treated with salmeterol withdrew } \\
\text { from the study because of adverse events; however, these adverse events were considered by the } \\
\text { investigator to be unrelated to study drug (bilateral subcapsular cataracts and postsurgical infection)." } \\
\text { Website: SFCA3003: no fatal adverse events. No serious adverse events in placebo arm; three in } \\
\text { salmeterol arm (asthma exacerbation, post surgical infection and chest pain) }\end{array}$ \\
\hline Notes & Funding: sponsored by GSK \\
\hline Risk of bias & \\
\hline
\end{tabular}




\begin{tabular}{lll} 
Bias & Authors' judgement & Support for judgement \\
\hline $\begin{array}{l}\text { Allocation } \\
\text { concealment } \\
\text { (selection bias) }\end{array}$ & Unclear risk & No information \\
\hline $\begin{array}{l}\text { Blinding } \\
\text { (performance } \\
\text { bias and } \\
\text { detection bias) }\end{array}$ & All outcomes & Low risk Double blind \\
\hline $\begin{array}{l}\text { Selective } \\
\text { reporting } \\
\text { (reporting }\end{array}$ & Low risk & \\
bias) & & Full SAE data on GSK trials register \\
\hline
\end{tabular}

\section{Simons 1997}

\begin{tabular}{|c|c|}
\hline Methods & Parallel group multicentre study from Canada, 2 week run in/52 weeks treatment/2 week run out \\
\hline Participants & $\begin{array}{l}241 \text { children. Mean age } 9.3 \text { mths with mild-moderate persistent asthma } \\
\text { Inclusion criteria: clinical stable asthma, atopic, well controlled, baseline } \mathrm{FEV}_{1}>70 \% \text { predicted, }> \\
10 \% \mathrm{FEV}_{1} \text { reversibility to SABA. PC } 20 \text { methacholine }<8 \mathrm{mg} / \mathrm{ml} \text { Exclusion: use of ICS/OS for }>4 \\
\text { weeks previously, OS/ICS within } 3 \text { mths exacerbation }<12 \text { weeks }\end{array}$ \\
\hline Interventions & $\begin{array}{l}\text { Salmeterol } 50 \mu \mathrm{g} \text { twice daily versus placebo (Diskus). ICS was not permitted as it was used as a } \\
\text { third comparison arm in this study }\end{array}$ \\
\hline Outcomes & $\begin{array}{l}\text { Effect on growth reported as adverse event, but no information in paper on fatal or nonfatal serious } \\
\text { adverse events } \\
\text { Website: SMS } 40065(521 / 120 \text { [SLPT10]). No fatal SAE. Salmeterol } 7 \text { subjects with non-fatal SAE, } \\
\text { placebo } 3 \text { subjects with non-fatal SAE }\end{array}$ \\
\hline Notes & Funding: supported by a grant from GSK \\
\hline \multicolumn{2}{|l|}{ Risk of bias } \\
\hline Bias & Support for judgement \\
\hline $\begin{array}{l}\text { Allocation } \\
\text { concealment } \\
\text { (selection bias) }\end{array}$ & No information \\
\hline $\begin{array}{l}\text { Blinding } \\
\text { (performance } \\
\text { bias and } \\
\text { detection bias) } \\
\text { All outcomes }\end{array}$ & Double blind \\
\hline $\begin{array}{l}\text { Selective } \\
\text { reporting } \\
\text { (reporting bias) }\end{array}$ & Full SAE data on GSK trials register \\
\hline
\end{tabular}

\section{SLGA 3014}

\begin{tabular}{|c|c|}
\hline Methods & Parallel group, multicentre (36) USA, 12-week treatment \\
\hline Participants & $\begin{array}{l}449 \text { children aged } 4 \text { to } 11 \text { years (mean age } 8 \text { years) } \\
\text { Inclusion: diagnosis asthma by ATS criteria, Baseline } \mathrm{FEV}_{1} / \mathrm{PEF} 45 \%-80 \% \text { predicted } \\
\text { Exclusion: not mentioned but reported as similar to Weinstein } 1998 \text { (URTI/LRTI/hospitalisation }<4 \\
\text { weeks, OS, anticholinergics, theophyllines, antihistamines, tobacco exposure, serious uncontrolled } \\
\text { systemic disease) }\end{array}$ \\
\hline Interventions & $\begin{array}{l}4 \text { arm study. Salmeterol } 50 \mu \mathrm{g} \text { twice daily, salmeterol } 25 \mu \mathrm{g} \text { twice daily, albuterol } 200 \mu \mathrm{g} \text { four times } \\
\text { daily versus placebo (all using dry powder devices - diskus/rotahaler) Co-interventions: ICS around } \\
50 \% \text {, cromones } 25 \% \text {, immunotherapy continued at same dose }\end{array}$ \\
\hline
\end{tabular}

Cochrane Database Syst Rev. Author manuscript; available in PMC 2014 May 09. 
Outcomes No website information or paper publication

Information from FDA submission: no deaths reported during this trial. Serious adverse events were reported in eight patients, one albuterol patient, three $25 \mu \mathrm{g}$ Diskus patients and four $50 \mu \mathrm{g}$ Diskus patients. The albuterol patient and three Diskus patients experienced asthma exacerbations

\begin{tabular}{lll}
\hline Notes & Funding: sponsored by GSK. & \\
\hline Risk of bias & & Support for judgement \\
\hline Bias & Authors' judgement & No information \\
\hline $\begin{array}{l}\text { Allocation } \\
\text { concealment } \\
\text { (selection bias) }\end{array}$ & Unclear risk & \\
\hline $\begin{array}{l}\text { Blinding } \\
\text { (performance } \\
\text { bias and } \\
\text { detection bias) }\end{array}$ & Low risk & Double-blind, double dummy \\
\hline $\begin{array}{l}\text { All outcomes } \\
\begin{array}{l}\text { Selective } \\
\text { reporting } \\
\text { (reporting bias) }\end{array}\end{array}$ & Low risk & $\begin{array}{l}\text { Mortality and SAE reported in FDA submission, } \\
\text { results not found on GSK trial register }\end{array}$ \\
\hline
\end{tabular}

\section{SLMF4002}

\begin{tabular}{|c|c|}
\hline Methods & $\begin{array}{l}\text { Multicentre, randomised, double-blind, placebo-controlled study } \\
\text { November } 1998 \text { to January } 2000\end{array}$ \\
\hline Participants & $\begin{array}{l}56 \text { centres in France } \\
\text { Main inclusion criteria for entry in run-in period: } \\
\text { males and non-pregnant, non-breastfeeding females, aged } 18 \text { years or over with asthma currently } \\
\text { controlled with ICS (beclometasone } 800 \text { to } 1200 \mu \mathrm{g} / \text { day or equivalent, or fluticasone } 500 \mu \mathrm{g} / \mathrm{day} \text { ) } \\
\text { without change with the last three months; bronchodilator treatment in the previous month as a short- } \\
\text { acting beta- }{ }_{2} \text { agonist (or beta- }-2 \text { agonist in combination with anticholinergics) } \\
\text { Main inclusion criteria for randomisation were a controlled asthma confirmed during the run-in } \\
\text { period, with, during the last week: morning PEF } 80 \% \text { of predicted value, and bronchodilator } \\
\text { requirement less than } 3 \text { times within one day or within several days, and no more than one day with } \\
\text { PEF variation > } 20 \% \\
\text { Main exclusion criteria were: a hospitalisation for asthma exacerbation in the previous year or a } \\
\text { lower respiratory tract infection in the previous month or, in the previous month, a treatment with } \\
\text { long acting beta- } 2 \text { agonist, fixed-dose of short-acting beta- } 2 \text { agonist or an anticholinergic, an anti- } \\
\text { leukotriene, or theophylline, or, in the last three months, a treatment with corticosteroids by general } \\
\text { route, or an extended release corticosteroid }\end{array}$ \\
\hline Interventions & $\begin{array}{l}\text { Salmeterol } 50 \mu \mathrm{g} / \text { inhalation }(\mathrm{N}=93) \text {, or matching placebo }(\mathrm{N}=95) \text { twice daily during } 6 \text { months } \\
\text { The primary objective was to evaluate the efficacy of sal } 100 \mu \mathrm{g} \text { per day in helping maintenance of } \\
\text { asthma control when ICS dosage is halved in subjects who were receiving } 1000 \mu \mathrm{g} \text { /day of } \\
\text { beclometasone or equivalent } \\
\text { Co-interventions: ICS } 100 \% \text { as this was an inclusion criterion but the dose was halved as part of the } \\
\text { protocol }\end{array}$ \\
\hline Outcomes & $\begin{array}{l}\text { Unpublished } \\
\text { Website: SLMF } 4002 \text {. No subjects with fatal SAE. Non-fatal SAE in } 1 \text { subject in the salmeterol group } \\
\text { and } 4 \text { subjects in the placebo group }\end{array}$ \\
\hline Notes & Funding: sponsored by GSK \\
\hline \multicolumn{2}{|l|}{ Risk of bias } \\
\hline Bias & Support for judgement \\
\hline $\begin{array}{l}\text { Allocation } \\
\text { concealment } \\
\text { (selection bias) }\end{array}$ & No information \\
\hline
\end{tabular}




\begin{tabular}{lll}
$\begin{array}{l}\text { Blinding } \\
\text { (performance } \\
\text { bias and } \\
\text { detection bias) }\end{array}$ & Low risk & Double blind \\
All outcomes & & \\
\hline $\begin{array}{l}\text { Selective } \\
\text { reporting } \\
\text { (reporting bias) }\end{array}$ & Low risk & Full SAE data on GSK trials register \\
\hline
\end{tabular}

SMART 2006

\begin{tabular}{|c|c|c|}
\hline Methods & \multicolumn{2}{|c|}{28 week parallel group study in 6163 centres in the USA } \\
\hline Participants & \multicolumn{2}{|c|}{$\begin{array}{l}\text { Randomised: } 26,355 \text {. Completed 19,128 (73\%). Mean age: } 39 \text { years } \\
\text { Patient characteristics at inclusion: mean PEFR: } 355 \text { L/min. Baseline ICS use } 47 \% \text { Inclusion } \\
\text { criteria: at least } 12 \text { years of age; diagnosis of asthma (clinical investigator); receiving current } \\
\text { prescription of asthma medication } \\
\text { Exclusion criteria: prior use of LABA; pregnancy or lactation; concurrent disease that may pose a } \\
\text { risk to the participant; sensitivity to long-acting beta-agonists; current ß-blocker use }\end{array}$} \\
\hline Interventions & \multicolumn{2}{|c|}{$\begin{array}{l}\text { Salmeterol } 50 \mu \mathrm{g} \text { twice daily verus placebo (MDI) } \\
\text { Co-interventions: ICS }(47 \%) \text {, methylxanthines, leukotriene agents }\end{array}$} \\
\hline Outcomes & \multicolumn{2}{|c|}{$\begin{array}{l}\text { Respiratory deaths and life-threatening events } \\
\text { Mortality (total, asthma-related, respiratory-related) } \\
\text { All cause hospitalisation } \\
\text { Combined all-cause death and life-threatening events, asthma-related death and life-threatening } \\
\text { events } \\
\text { Subgroups identified (post hoc): Caucasian/African American participants; ICS at baseline/no ICS at } \\
\text { baseline; predicted PEF above or below } 60 \% \\
\text { Paper report: "Overall, } 1093 \text { subjects ( } 4 \% \text { in each treatment group) had serious adverse events } \\
\text { during the study" } \\
\text { Website SGLA5011: } 580 \text { fatal and non-fatal SAEs with salmeterol and } 513 \text { with placebo }\end{array}$} \\
\hline Notes & \multicolumn{2}{|c|}{ Funding: sponsored by GSK } \\
\hline \multicolumn{3}{|l|}{ Risk of bias } \\
\hline Bias & Authors' judgement & Support for judgement \\
\hline $\begin{array}{l}\text { Allocation } \\
\text { concealment } \\
\text { (selection bias) }\end{array}$ & Unclear risk & No information \\
\hline $\begin{array}{l}\text { Blinding } \\
\text { (performance } \\
\text { bias and } \\
\text { detection bias) } \\
\text { All outcomes }\end{array}$ & Low risk & $\begin{array}{l}\text { Blinding of participants, personnel and outcomes: } \\
\text { placebo MDI used. Morbidity and Mortality } \\
\text { Review Committee were blinded to study treatment }\end{array}$ \\
\hline $\begin{array}{l}\text { Selective } \\
\text { reporting } \\
\text { (reporting bias) }\end{array}$ & Low risk & Full SAE data on GSK trials register \\
\hline
\end{tabular}

SNS 1993

\begin{tabular}{|c|c|}
\hline Methods & Parallel group, double-blind 16 week study in UK Primary Care (3516 GPs) \\
\hline Participants & $\begin{array}{l}25,180 \text { adults and adolescents (age range } 12 \text { to }>60 \text { years) } \\
\text { Inclusion: clinical diagnosis of asthma requiring regular bronchodilator } \\
\text { Exclusion: beta blocker use, serious uncontrolled diseases, pregnancy }\end{array}$ \\
\hline Interventions & $\begin{array}{l}\text { Salmeterol } 50 \mu \mathrm{g} \text { twice with placebo at noon and early evening versus salbutamol } 200 \mu \mathrm{g} \text { four times } \\
\text { daily (given by MDI) } \\
\text { Co-interventions: ICS } 69 \% \text {, OS } 4.7 \% \text {, theophyllines, chromones, ipratropium - stable doses }\end{array}$ \\
\hline
\end{tabular}

Cochrane Database Syst Rev. Author manuscript; available in PMC 2014 May 09. 


\begin{tabular}{lll} 
Outcomes & $\begin{array}{l}\text { Deaths, admission to hospital/life-threatening events. Other serious adverse events and withdrawals. } \\
\text { Each outcome was subdivided into respiratory related to asthma and respiratory unrelated to asthma } \\
\text { and non-respiratory causes. If patients suffered more than one adverse event they were counted } \\
\text { under the most severe category } \\
\text { Results expressed as absolute incidence and relative risks } \\
\text { Website: SNS-D920619. Data on all mortality and serious adverse events }\end{array}$ \\
\hline Notes & $\begin{array}{l}\text { Funding: sponsored by GSK. No published inhaled corticosteroid usage in relation to outcomes, but } \\
\text { data obtained from GSK for baseline steroid use in those with asthmarelated deaths }\end{array}$ \\
\hline Risk of bias & & \\
\hline Bias & Authors' judgement & Support for judgement \\
\hline $\begin{array}{l}\text { Allocation } \\
\text { concealment } \\
\text { (selection bias) }\end{array}$ & Low risk & $\begin{array}{l}\text { Computer generated random code, blocks of } 6 . \\
\text { Prenumbered treatment packs given to the patients as } \\
\text { they were allocated the next consecutive study } \\
\text { number }\end{array}$ \\
\hline $\begin{array}{l}\text { Blinding } \\
\text { (performance } \\
\text { bias and } \\
\text { detection bias) } \\
\text { All outcomes }\end{array}$ & Low risk & $\begin{array}{l}\text { Double blind, double dummy, matching devices. } \\
\text { Independent consultants who reviewed deaths } \\
\text { possibly related to asthma were blinded to treatment } \\
\text { group }\end{array}$ \\
\hline $\begin{array}{l}\text { Selective } \\
\text { reporting } \\
\text { (reporting bias) }\end{array}$ & Low risk & Full SAE data on GSK trials register \\
\hline & & \\
\hline
\end{tabular}

Von Berg 1998

\begin{tabular}{|c|c|}
\hline Methods & $\begin{array}{l}\text { Parallel group, multicentre (57) in } 11 \text { countries, } 2 \text { week run in and } 52 \text { week treatment with } 2 \text { week } \\
\text { period off treatment at } 6 \mathrm{mths} \text { to assess BHR }\end{array}$ \\
\hline Participants & $\begin{array}{l}426 \text { children, mean age } 10 \text { years (range } 5-15 \text { ) } \\
\text { Inclusion: clinically diagnosed asthma, }>15 \% \mathrm{FEV}_{1} \text { reversibility to SABA or diurnal variation } \\
\mathrm{PEF}>15 \% \text {, am PEF }<55 \% \text { best in run in } \\
\text { Exclusion:URTI/LRTI/hospitalisation/changed asthma medication }<4 \text { weeks, requiring OS/ } \\
\text { anticholinergics/methylxanthines at entry }\end{array}$ \\
\hline Interventions & $\begin{array}{l}\text { Salmeterol } 50 \mu \mathrm{g} \text { twice daily versus placebo (diskhaler) } \\
\text { Co-interventions: ICS } 50 \% \text {, cromones } 22 \%\end{array}$ \\
\hline Outcomes & $\begin{array}{l}\text { Paper publication mentions frequent and drug-related adverse events but no report of serious } \\
\text { adverse events } \\
\text { Website SLGB3019 (SLPT09): no fatal adverse events over the entire study period. Data averaged } \\
\text { from the two periods so } 18 \text { in salmeterol group and } 13 \text { in placebo group on average over each six } \\
\text { months }\end{array}$ \\
\hline Notes & Funding: sponsored by GSK \\
\hline \multicolumn{2}{|l|}{ Risk of bias } \\
\hline Bias & Support for judgement \\
\hline $\begin{array}{l}\text { Allocation } \\
\text { concealment } \\
\text { (selection bias) }\end{array}$ & No information \\
\hline $\begin{array}{l}\text { Blinding } \\
\text { (performance } \\
\text { bias and } \\
\text { detection bias) } \\
\text { All outcomes }\end{array}$ & Double blind \\
\hline $\begin{array}{l}\text { Selective } \\
\text { reporting } \\
\text { (reporting bias) }\end{array}$ & Full SAE data on GSK trials register \\
\hline
\end{tabular}

\section{Weinstein 1998}

Cochrane Database Syst Rev. Author manuscript; available in PMC 2014 May 09. 


\begin{tabular}{|c|c|c|}
\hline Methods & \multicolumn{2}{|c|}{ Parallel group, multicentre (11) USA, 1-2 week run in on single blind placebo/12 week treatment } \\
\hline Participants & \multicolumn{2}{|c|}{$\begin{array}{l}207 \text { children, mean age } 8.4 \text { years (range } 4-11 \text { ) } \\
\text { Inclusion: diagnosis asthma by ATS criteria, Baseline } \mathrm{FEV}_{1} 50 \%-80 \% \text { predicted, }>15 \% \mathrm{FEV}_{1} \\
\text { reversibility to SABA } \\
\text { Exclusion: URTI/LRTI/hospitalisation < } 4 \text { weeks, OS, anticholinergics, theophyllines, } \\
\text { antihistamines, tobacco exposure, serious uncontrolled systemic disease }\end{array}$} \\
\hline Interventions & \multicolumn{2}{|c|}{$\begin{array}{l}\text { Salmeterol } 50 \mu \mathrm{g} \text { twice daily versus placebo } \\
\text { Co-interventions: ICS } 57 \% \text {, cromones } 32 \% \text {, immunotherapy continued at same dose }\end{array}$} \\
\hline Outcomes & \multicolumn{2}{|c|}{$\begin{array}{l}\text { Paper reports "Four salmeterol- and five placebo-treated patients experienced serious adverse } \\
\text { events requiring hospitalisation. These included asthma exacerbation (four salmeterol, two } \\
\text { placebo), appendicitis (two placebo), and kidney obstruction and dehydration (one placebo)" } \\
\text { No website data for this trial on GSK site (SLD390). Listed on FDA site submission from GSK }\end{array}$} \\
\hline Notes & \multicolumn{2}{|c|}{ Funding: sponsored by GSK } \\
\hline \multicolumn{3}{|l|}{ Risk of bias } \\
\hline Bias & Authors' judgement & Support for judgement \\
\hline $\begin{array}{l}\text { Allocation } \\
\text { concealment } \\
\text { (selection bias) }\end{array}$ & Unclear risk & No information \\
\hline $\begin{array}{l}\text { Blinding } \\
\text { (performance } \\
\text { bias and } \\
\text { detection bias) } \\
\text { All outcomes }\end{array}$ & Low risk & Double blind, double dummy \\
\hline $\begin{array}{l}\text { Selective } \\
\text { reporting } \\
\text { (reporting bias) }\end{array}$ & Low risk & $\begin{array}{l}\text { Not found in GSK trial register, but SAE data } \\
\text { given in paper }\end{array}$ \\
\hline
\end{tabular}

Wenzel 1998

\begin{tabular}{|c|c|c|}
\hline Methods & \multicolumn{2}{|c|}{ Parallel group, multicentre, 40 , USA. 12 weeks } \\
\hline Participants & \multicolumn{2}{|c|}{$\begin{array}{l}539 \text { adults/adolescents with mean age: } 35.4 \text { (range } 12 \text { to } 83 \text { ) } \\
\text { Inclusion: diagnosis asthma by ATS criteria, requiring daily bronchodilator treatment }>6 \text { weeks. } \\
\text { Baseline FEV } 40 \% \text { to } 80 \% \text { predicted, }>15 \% \mathrm{FEV}_{1} \text { reversibility to SABA } \\
\text { Exclusion: CF, COPD, current smokers, hospitalised by exacerbation asthma < } 6 \text { weeks. } \\
\text { Use of theophyllines or cromones }\end{array}$} \\
\hline Interventions & \multicolumn{2}{|c|}{$\begin{array}{l}\text { Salmeterol } 50 \mu \mathrm{g} \text { twice daily with placebo twice daily versus salbutamol } 200 \mu \mathrm{g} \text { four times daily } \\
\text { Co-interventions: ICS } 46 \% \text { (no other details regarding co-interventions) }\end{array}$} \\
\hline Outcomes & \multicolumn{2}{|c|}{$\begin{array}{l}\text { No website data found for this study. Further information requested from GSK } \\
\text { Paper report: "Three patients experienced serious adverse events...Two patients treated with } \\
\text { salmeterol experienced status asthmaticus; one event was considered to be potentially related to the } \\
\text { study drug while the other was not. The other serious adverse event that occurred in one patient } \\
\text { treated with albuterol was antisocial behavior and was not considered to be related to study drug" }\end{array}$} \\
\hline Notes & \multicolumn{2}{|c|}{ Funding: supported by a grant from GSK } \\
\hline \multicolumn{3}{|l|}{ Risk of bias } \\
\hline Bias & Authors' judgement & Support for judgement \\
\hline $\begin{array}{l}\text { Allocation } \\
\text { concealment } \\
\text { (selection bias) }\end{array}$ & Unclear risk & No information \\
\hline $\begin{array}{l}\text { Blinding } \\
\text { (performance } \\
\text { bias and } \\
\text { detection bias) } \\
\text { All outcomes }\end{array}$ & Low risk & Double blind, double dummy, matching devices \\
\hline
\end{tabular}

Cochrane Database Syst Rev. Author manuscript; available in PMC 2014 May 09. 


\begin{tabular}{lll}
$\begin{array}{l}\text { Selective } \\
\text { reporting } \\
\text { (reporting bias) }\end{array}$ & Low risk & $\begin{array}{l}\text { Not found in GSK trial register, but all-cause SAE } \\
\text { data published in paper }\end{array}$ \\
\hline
\end{tabular}

Wolfe 2000

\begin{tabular}{|c|c|}
\hline Methods & $\begin{array}{l}\text { Parallel group. Combined data from two studies with } 3 \text { arms comparing } 2 \text { forms of salmeterol } \\
\text { (powder and aerosol) and placebo, multicentre (27) USA. } 2 \text { week run in period/12-week treatment } \\
\text { period }\end{array}$ \\
\hline Participants & $\begin{array}{l}498 \text { adults/ adolescents, with mean age } 33 \text { years (range } 12-79) \\
\text { Inclusion : diagnosis asthma by ATS criteria } 6 \text { mths requiring pharmacotherapy. Baseline } \mathrm{FEV}_{1}> \\
85 \% \text { predicted, }>15 \% \mathrm{FEV} \mathrm{F}_{1} \text { reversibility to SABA } \\
\text { Exclusion : URTI/LRTI/exacerbation }<6 \text { weeks, smoker or }>10 \text { year pack history }\end{array}$ \\
\hline Interventions & $\begin{array}{l}\text { Salmeterol } 50 \mu \mathrm{g} \text { twice daily via accuhaler or MDI versus placebo in same device } \\
\text { Co-interventions: ICS }>30 \% \text { all groups, chromones continued at same dose }\end{array}$ \\
\hline Outcomes & $\begin{array}{l}\text { Paper publication "Overall there were no statistically significant between-group differences in the } \\
\text { incidence of drug-related adverse events" } \\
\text { Website SLGA3010: no subjects with fatal SAE, non-fatal SAE in } 5 \text { subjects on salmeterol ( } 3 \\
\text { asthma and } 2 \text { bronchitis) and one on placebo (pneumonia) } \\
\text { Website SLGA3011: no subjects with fatal SAE, non-fatal SAE in } 1 \text { subject on salmeterol } \\
\text { (cholecystitis) and none on placebo }\end{array}$ \\
\hline Notes & Funding: sponsored by GSK \\
\hline \multicolumn{2}{|l|}{ Risk of bias } \\
\hline Bias & Support for judgement \\
\hline $\begin{array}{l}\text { Allocation } \\
\text { concealment } \\
\text { (selection bias) }\end{array}$ & No information \\
\hline $\begin{array}{l}\text { Blinding } \\
\text { (performance } \\
\text { bias and } \\
\text { detection bias) } \\
\text { All outcomes }\end{array}$ & Double blind, double dummy \\
\hline $\begin{array}{l}\text { Selective } \\
\text { reporting } \\
\text { (reporting bias) }\end{array}$ & Full SAE data on GSK trials register \\
\hline
\end{tabular}

ATS: American Thoracic Society

bd: Bis die (Latin for 'twice a day')

BDP: beclomethasone dipropionate

BHR: bronchial hyperresponsiveness

$\mathrm{CF}$ : cystic fibrosis

CFC: chlorofluorocarbon

COPD: chronic obstructive pulmonary disease

CXR: chest X-ray

D \& V: diarrhoea and vomiting

DPI: dry powder inhaler

ECG: electrocardiogram

FDA: Food \& Drug Administration

FP: fluticasone propionate

GI: gastrointestinal

HFA: hydrofluoroalkane 
ICS: inhaled corticosteroids

IM: intramuscular

LABA: long-acting beta2-agonist

LRTI: lower respiratory tract infection

MDI: metered dose inhaler

OCS: oral corticosteroid

PEF: peak expiratory flow

PEFR: peak expiratory flow rate

PFT: pulmonary function test

prn: pro re nata (Latin for 'taken as needed')

QID: Quarter in die (Latin for 'four times each day')

RAO: reversible airways obstruction

RTI: respiratory tract infection

SAE: serious adverse event

SAL: salmeterol

TAA: triamcinolone acetonide

TIA: transient ischemic attack

URTI: upper respiratory tract infection

\section{Characteristics of excluded studies}

\begin{tabular}{ll}
\hline Study & Reason for exclusion \\
\hline Bagnato 1996 & Single dose study \\
\hline Beach 1992 & Single dose study \\
\hline Beach 1993 & 6 week duration \\
\hline Blake 1999 & Single dose study \\
\hline Bons 1992 & Overview of other studies \\
\hline Booth 1993 & 8 week duration \\
\hline Boulet 1997b & 8 week duration \\
\hline Bousquet 1996 & 8 week duration \\
\hline Brambilla 1994 & 2 week duration \\
\hline Bronsky 1994 & 1 week duration \\
\hline Bronsky 1999 & Single dose study \\
\hline Busse 1999 & 4 week duration \\
\hline Cartier 1993 & Single dose study \\
\hline Castle 1992 & Not RCT \\
\hline Cazzola 2002 & Patients with COPD \\
\hline Ceugniet 1997 & Single dose \\
\hline Cheung 1992 & 8 week duration \\
\hline Chopra 2005 & Comparison of propellants \\
\hline Cloosterman 2001 & Formoterol not salmeterol used \\
\hline Crompton 1999 & 6 week duration \\
\hline D'Alonzo 1995 & Review of D'Alonzo 1994 and Pearlman 1992 \\
\hline
\end{tabular}

Cochrane Database Syst Rev. Author manuscript; available in PMC 2014 May 09. 


\begin{tabular}{|c|c|}
\hline D’Urzo 1998 & Not RCT \\
\hline Dahl 1991 & 4 week duration \\
\hline De Oliveira 1998 & 4 week duration \\
\hline Dekhuijzen 2006 & Not RCT \\
\hline Demirkan 2000 & Comparison of delivery devices \\
\hline Deykin 2007 & Combination therapy with salmeterol and LRTA \\
\hline Edelman 2000 & 8 week duration \\
\hline Eryonucu 2005 & Single dose study \\
\hline Faurschou 1994 & 3 week duration \\
\hline Faurschou 1996 & 6 week duration \\
\hline Fjellbirkeland 1994 & 2 week duration \\
\hline Fuller 1995 & Not RCT (overview of safety data) \\
\hline GlaxoSmithKline 2005 & Single dose study \\
\hline Gongora 1991 & Single dose study \\
\hline Gotz 1995 & Crossover study \\
\hline Gustafsson 1994 & Comparison of delivery devices \\
\hline Harper 2001 & 5 week study duration \\
\hline Hermansson 1995 & 4 week duration \\
\hline Inoue 2007 & Single dose crossover study \\
\hline Jartti 1998 & 4 week duration \\
\hline Jenkins 1991 & Not RCT (time series data of exacerbations) \\
\hline Johnson 1994 & 6 week duration \\
\hline Kemp 1993 & Single dose study \\
\hline Kirby 1995 & Not on asthma (healthy volunteers) \\
\hline Kraemer 1997 & Comparison of delivery devices \\
\hline Kurihara 1993 & Comparison of delivery devices \\
\hline Langley 1998 & 4 week duration \\
\hline Langton Hewer 1995 & 8 week treatment period \\
\hline Lemaigre 2006 & Single dose study \\
\hline Lemanske 2001 & Patients randomised to salmeterol and TAA and then TAA was withdrawn in one arm \\
\hline Lotvall 1998 & Not asthma \\
\hline Lurie 2005 & Not RCT \\
\hline Martin 1999 & 3 week duration \\
\hline Meier 1997 & Not RCT (cohort study) \\
\hline Mikawa 1993 & 2 week duration \\
\hline Miyamoto 1993a & 4 week duration \\
\hline Miyamoto $1993 b$ & 4 week duration \\
\hline Miyamoto $1993 \mathrm{c}$ & Single dose \\
\hline Miyamoto $1993 \mathrm{~d}$ & Single dose \\
\hline Miyamoto $1993 \mathrm{e}$ & 4 week duration \\
\hline
\end{tabular}

Cochrane Database Syst Rev. Author manuscript; available in PMC 2014 May 09. 


\begin{tabular}{|c|c|}
\hline Morgan 1994 & Single dose study \\
\hline Muir 1992 & 4 week duration \\
\hline Nathan 1995 & Review of D'Alonzo 1994 and Pearlman 1992 \\
\hline Nelson 1999 & 4 week duration \\
\hline Nelson 2001 & Comparison with LRTA \\
\hline Nishima 1993 & Single dose \\
\hline Nishiyama 2006 & Comparison with tulobuterol \\
\hline Norhaya 1999 & 4 week duration, cross-over \\
\hline Nutini 1998 & Comparison with theophylline \\
\hline Orgel 1985 & Single dose study \\
\hline Ortiz 2002 & Single dose study \\
\hline Paggiaro 1996 & Comparison with theophylline \\
\hline Palmer 1992 & Dose response study \\
\hline Pascoe 2006 & Crossover study \\
\hline Pastorello 1998 & Comparison with theophylline \\
\hline Pearlman 1999 & 4 week duration \\
\hline Peslis 1994 & Comparison with fenoterol \\
\hline Peters 2000 & Short-term study in hospitalised patients \\
\hline Pohunek 2004 & Single dose study \\
\hline Pollard 1997 & Comparison to theophylline \\
\hline Prieto 2002 & 6 week duration \\
\hline Revill 1998 & Exercise-induced bronchospasm \\
\hline Rhee 1997 & 6 week duration \\
\hline Ringbaek 1996 & 4 week duration \\
\hline Ringdal 1995 & 4 week duration, propellant comparison \\
\hline Ritz 1997 & Short term study on patients in ITU \\
\hline Roberts 1999 & 6 week duration \\
\hline Sano 1993 & Single dose study \\
\hline Schaanning 1996 & Exercise-induced bronchospasm \\
\hline Shaheen 1994 & Single dose, propellant comparison \\
\hline Shepherd 1991 & Overview of other studies \\
\hline SLGL24 & 8 week randomised treatment period \\
\hline Stahl 1999 & 6 week duration \\
\hline Starke 1996 & 4 week duration, cross-over \\
\hline Storms 2004 & 4 week duration \\
\hline Szczeklik 1998 & Single dose study \\
\hline Taguchi 1993 & 8 week duration \\
\hline Taylor 1992 & Single dose study \\
\hline Taylor 1998 & Cross-over study \\
\hline Taylor 2000a & Not RCT \\
\hline
\end{tabular}

Cochrane Database Syst Rev. Author manuscript; available in PMC 2014 May 09. 


\begin{tabular}{ll}
\hline Taylor 2000b & 2 week cross-over study \\
\hline Thompson 1994 & No control arm for open label extension reported in this abstract \\
\hline Tomac 1996 & 4 week duration \\
\hline Ukena 1997 & Cross-over study, comparison with theophylline \\
\hline Ullman 1990 & 2 week duration \\
\hline Venables 1992 & 4 week duration, cross-over \\
\hline Verberne 1993 & Single dose study \\
\hline Verberne 1998 & All patients randomised to ICS and salmeterol \\
\hline Verini 1998 & 1 week duration \\
\hline Villaran 1999 & Exercise-induced bronchospasm \\
\hline Weersink 1997 & 6 week duration \\
\hline Weiner 2003 & Single dose study \\
\hline Weinstein 1997 & 1 week duration \\
\hline Wiegand 1999 & Comparison with theophylline \\
\hline Wilding 1997 & Cross-over study \\
\hline Williams 1998 & Not RCT (case control study) \\
\hline Zarkovic 1998 & Cross-over study \\
\hline Zimmermann 2003 & Exercise-induced bronchospasm \\
\hline
\end{tabular}

ICS: inhaled corticosteroids; ITU: intensive therapy unit/intensive treatment unit; LRTA: leukotriene receptor antagonist; TAA:

\section{DATA AND ANALYSES}

Comparison 1

Regular salmeterol versus placebo or regular salbutamol

\begin{tabular}{|c|c|c|c|c|}
\hline Outcome or subgroup title & No. of studies & No. of participants & Statistical method & Effect size \\
\hline 1 All-cause mortality & 20 & & $\begin{array}{l}\text { Peto Odds Ratio } \\
\text { (Peto, Fixed, 95\% CI) }\end{array}$ & Subtotals only \\
\hline $\begin{array}{l}\text { 1.1 Salmeterol versus } \\
\text { placebo }\end{array}$ & 14 & 30254 & $\begin{array}{l}\text { Peto Odds Ratio } \\
\text { (Peto, Fixed, 95\% CI) }\end{array}$ & $1.33[0.85,2.08]$ \\
\hline $\begin{array}{l}\text { 1.2 Salmeterol versus } \\
\text { salbutamol }\end{array}$ & 7 & 27615 & $\begin{array}{l}\text { Peto Odds Ratio } \\
\text { (Peto, Fixed, 95\% CI) }\end{array}$ & $1.22[0.76,1.96]$ \\
\hline $\begin{array}{l}2 \text { Non-fatal serious adverse } \\
\text { events (adults and children) }\end{array}$ & 25 & & $\begin{array}{l}\text { Peto Odds Ratio } \\
\text { (Peto, Fixed, 95\% CI) }\end{array}$ & Subtotals only \\
\hline $\begin{array}{l}\text { 2.1 Salmeterol versus } \\
\text { placebo }\end{array}$ & 18 & 31529 & $\begin{array}{l}\text { Peto Odds Ratio } \\
\text { (Peto, Fixed, 95\% CI) }\end{array}$ & $1.15[1.02,1.29]$ \\
\hline $\begin{array}{l}\text { 2.2 Salmeterol versus } \\
\text { salbutamol }\end{array}$ & 8 & 28183 & $\begin{array}{l}\text { Peto Odds Ratio } \\
\text { (Peto, Fixed, 95\% CI) }\end{array}$ & $0.96[0.81,1.14]$ \\
\hline $\begin{array}{l}3 \text { Non-fatal serious adverse } \\
\text { events in adults }\end{array}$ & 18 & & $\begin{array}{l}\text { Peto Odds Ratio } \\
\text { (Peto, Fixed, 95\% CI) }\end{array}$ & Subtotals only \\
\hline $\begin{array}{l}\text { 3.1 Salmeterol versus } \\
\text { placebo in adults }\end{array}$ & 13 & 30196 & $\begin{array}{l}\text { Peto Odds Ratio } \\
\text { (Peto, Fixed, 95\% CI) }\end{array}$ & $1.14[1.01,1.28]$ \\
\hline $\begin{array}{l}\text { 3.2 Salmeterol versus } \\
\text { salbutamol in adults }\end{array}$ & 5 & 27002 & $\begin{array}{l}\text { Peto Odds Ratio } \\
\text { (Peto, Fixed, 95\% CI) }\end{array}$ & $0.94[0.79,1.11]$ \\
\hline
\end{tabular}




\begin{tabular}{|c|c|c|c|c|}
\hline Outcome or subgroup title & No. of studies & No. of participants & Statistical method & Effect size \\
\hline $\begin{array}{l}4 \text { Non-fatal serious adverse } \\
\text { events in children }\end{array}$ & 7 & & $\begin{array}{l}\text { Peto Odds Ratio } \\
\text { (Peto, Fixed, 95\% CI) }\end{array}$ & Subtotals only \\
\hline $\begin{array}{l}\text { 4.1 Salmeterol versus } \\
\text { placebo in children }\end{array}$ & 5 & 1333 & $\begin{array}{l}\text { Peto Odds Ratio } \\
\text { (Peto, Fixed, 95\% CI) }\end{array}$ & $1.30[0.82,2.05]$ \\
\hline $\begin{array}{l}\text { 4.2 Salmeterol versus } \\
\text { salbutamol (from first } 3 \\
\text { months of Lenney) }\end{array}$ & 3 & 1181 & $\begin{array}{l}\text { Peto Odds Ratio } \\
\text { (Peto, Fixed, 95\% CI) }\end{array}$ & $1.37[0.71,2.64]$ \\
\hline $\begin{array}{l}\text { 4.3 Salmeterol versus } \\
\text { salbutamol (data from Lenney } \\
9 \text { month trial extensions) }\end{array}$ & 3 & 1130 & $\begin{array}{l}\text { Peto Odds Ratio } \\
\text { (Peto, Fixed, 95\% CI) }\end{array}$ & $1.17[0.71,1.94]$ \\
\hline $\begin{array}{l}5 \text { Non-fatal adverse events in } \\
\text { adults and children (SNS } \\
\text { sensitivity analysis) }\end{array}$ & 25 & & $\begin{array}{l}\text { Peto Odds Ratio } \\
\text { (Peto, Fixed, 95\% CI) }\end{array}$ & Subtotals only \\
\hline $\begin{array}{l}5.1 \text { Salmeterol versus } \\
\text { placebo }\end{array}$ & 18 & 31529 & $\begin{array}{l}\text { Peto Odds Ratio } \\
\text { (Peto, Fixed, 95\% CI) }\end{array}$ & $1.15[1.02,1.29]$ \\
\hline $\begin{array}{l}5.2 \text { Salmeterol versus } \\
\text { salbutamol }\end{array}$ & 8 & 28188 & $\begin{array}{l}\text { Peto Odds Ratio } \\
\text { (Peto, Fixed, 95\% CI) }\end{array}$ & $0.98[0.86,1.11]$ \\
\hline $\begin{array}{l}6 \text { Fatal and non-fatal serious } \\
\text { adverse events in adults and } \\
\text { children }\end{array}$ & 25 & & $\begin{array}{l}\text { Peto Odds Ratio } \\
\text { (Peto, Fixed, 95\% CI) }\end{array}$ & Subtotals only \\
\hline $\begin{array}{l}6.1 \text { Salmeterol versus } \\
\text { placebo }\end{array}$ & 18 & 31529 & $\begin{array}{l}\text { Peto Odds Ratio } \\
\text { (Peto, Fixed, 95\% CI) }\end{array}$ & $1.16[1.03,1.30]$ \\
\hline $\begin{array}{l}\text { 6.2 Salmeterol versus } \\
\text { salbutamol }\end{array}$ & 8 & 28183 & $\begin{array}{l}\text { Peto Odds Ratio } \\
\text { (Peto, Fixed, 95\% CI) }\end{array}$ & $0.99[0.84,1.16]$ \\
\hline 7 Asthma mortality & 2 & 51535 & $\begin{array}{l}\text { Peto Odds Ratio } \\
\text { (Peto, Fixed, 95\% CI) }\end{array}$ & $2.94[1.41,6.14]$ \\
\hline $\begin{array}{l}7.1 \text { Salmeterol versus } \\
\text { placebo }\end{array}$ & 1 & 26355 & $\begin{array}{l}\text { Peto Odds Ratio } \\
\text { (Peto, Fixed, 95\% CI) }\end{array}$ & $3.49[1.31,9.31]$ \\
\hline $\begin{array}{l}\text { 7.2 Salmeterol versus } \\
\text { salbutamol }\end{array}$ & 1 & 25180 & $\begin{array}{l}\text { Peto Odds Ratio } \\
\text { (Peto, Fixed, 95\% CI) }\end{array}$ & $2.36[0.78,7.16]$ \\
\hline $\begin{array}{l}8 \text { Asthma mortality (within } \\
\text { study subgroups by ICS use at } \\
\text { baseline) }\end{array}$ & 2 & 46650 & $\begin{array}{l}\text { Peto Odds Ratio } \\
\text { (Peto, Fixed, 95\% CI) }\end{array}$ & $2.90[1.38,6.13]$ \\
\hline 8.1 ICS used at baseline & 2 & 26969 & $\begin{array}{l}\text { Peto Odds Ratio } \\
\text { (Peto, Fixed, 95\% CI) }\end{array}$ & $1.49[0.54,4.11]$ \\
\hline 8.2 Not on ICS at baseline & 2 & 19681 & $\begin{array}{l}\text { Peto Odds Ratio } \\
\text { (Peto, Fixed, 95\% CI) }\end{array}$ & $6.43[2.13,19.42]$ \\
\hline $\begin{array}{l}9 \text { Asthma mortality (within } \\
\text { study subgroups by any steroid } \\
\text { at baseline) }\end{array}$ & 2 & 47550 & $\begin{array}{l}\text { Peto Odds Ratio } \\
\text { (Peto, Fixed, 95\% CI) }\end{array}$ & $2.95[1.41,6.14]$ \\
\hline $\begin{array}{l}9.1 \text { ICS or oral steroids used } \\
\text { at baseline }\end{array}$ & 2 & 27869 & $\begin{array}{l}\text { Peto Odds Ratio } \\
\text { (Peto, Fixed, 95\% CI) }\end{array}$ & $2.03[0.82,5.00]$ \\
\hline $\begin{array}{l}9.2 \text { Not any steroids at } \\
\text { baseline }\end{array}$ & 2 & 19681 & $\begin{array}{l}\text { Peto Odds Ratio } \\
\text { (Peto, Fixed, 95\% CI) }\end{array}$ & $6.15[1.73,21.84]$ \\
\hline 10 Cardiovascular mortality & 19 & & $\begin{array}{l}\text { Peto Odds Ratio } \\
\text { (Peto, Fixed, 95\% CI) }\end{array}$ & Subtotals only \\
\hline $\begin{array}{l}\text { 10.1 Salmeterol versus } \\
\text { placebo }\end{array}$ & 13 & 29920 & $\begin{array}{l}\text { Peto Odds Ratio } \\
\text { (Peto, Fixed, 95\% CI) }\end{array}$ & $0.75[0.32,1.77]$ \\
\hline $\begin{array}{l}\text { 10.2 Salmeterol versus } \\
\text { salbutamol }\end{array}$ & 6 & 27281 & $\begin{array}{l}\text { Peto Odds Ratio } \\
\text { (Peto, Fixed, 95\% CI) }\end{array}$ & $1.22[0.64,2.34]$ \\
\hline $\begin{array}{l}11 \text { Adults and Children non- } \\
\text { fatal asthma-related serious } \\
\text { adverse events }\end{array}$ & 22 & & $\begin{array}{l}\text { Peto Odds Ratio } \\
\text { (Peto, Fixed, 95\% CI) }\end{array}$ & Subtotals only \\
\hline $\begin{array}{l}\text { 11.1 Salmeterol versus } \\
\text { placebo }\end{array}$ & 17 & 5174 & $\begin{array}{l}\text { Peto Odds Ratio } \\
\text { (Peto, Fixed, 95\% CI) }\end{array}$ & $1.59[1.05,2.41]$ \\
\hline
\end{tabular}




\begin{tabular}{|c|c|c|c|c|}
\hline Outcome or subgroup title & No. of studies & No. of participants & Statistical method & Effect size \\
\hline $\begin{array}{l}\text { 11.2 Salmeterol versus } \\
\text { salbutamol }\end{array}$ & 6 & 2341 & $\begin{array}{l}\text { Peto Odds Ratio } \\
\text { (Peto, Fixed, 95\% CI) }\end{array}$ & $0.99[0.54,1.81]$ \\
\hline $\begin{array}{l}12 \text { Adults and children non- } \\
\text { fatal cardiovascular serious } \\
\text { adverse events }\end{array}$ & 21 & & $\begin{array}{l}\text { Peto Odds Ratio } \\
\text { (Peto, Fixed, 95\% CI) }\end{array}$ & Subtotals only \\
\hline $\begin{array}{l}\text { 12.1 Salmeterol versus } \\
\text { placebo }\end{array}$ & 15 & 30579 & $\begin{array}{l}\text { Peto Odds Ratio } \\
\text { (Peto, Fixed, 95\% CI) }\end{array}$ & $0.98[0.73,1.31]$ \\
\hline $\begin{array}{l}\text { 12.2 Salmeterol versus } \\
\text { placebo (US trial results) }\end{array}$ & 1 & 9275 & $\begin{array}{l}\text { Peto Odds Ratio } \\
\text { (Peto, Fixed, 95\% CI) }\end{array}$ & $0.90[0.27,2.97]$ \\
\hline $\begin{array}{l}\text { 12.3 Salmeterol versus } \\
\text { salbutamol }\end{array}$ & 5 & 26794 & $\begin{array}{l}\text { Peto Odds Ratio } \\
\text { (Peto, Fixed, 95\% CI) }\end{array}$ & $1.06[0.67,1.68]$ \\
\hline $\begin{array}{l}13 \text { Proportion of participants } \\
\text { with serious asthma } \\
\text { exacerbations in relation to } \\
\text { dose of ICS }\end{array}$ & 1 & 911 & $\begin{array}{l}\text { Peto Odds Ratio } \\
\text { (Peto, Fixed, 95\% CI) }\end{array}$ & $0.99[0.71,1.39]$ \\
\hline $\begin{array}{l}13.1 \text { ICS under } 500 \text { mcg per } \\
\text { day }\end{array}$ & 1 & 165 & $\begin{array}{l}\text { Peto Odds Ratio } \\
\text { (Peto, Fixed, 95\% CI) }\end{array}$ & $1.04[0.42,2.61]$ \\
\hline $\begin{array}{l}13.2 \text { ICS } 500 \text { to } 1000 \mathrm{mcg} \\
\text { per day }\end{array}$ & 1 & 537 & $\begin{array}{l}\text { Peto Odds Ratio } \\
\text { (Peto, Fixed, 95\% CI) }\end{array}$ & $0.68[0.42,1.08]$ \\
\hline $\begin{array}{l}\text { 13.3 ICS over } 1000 \text { mcg per } \\
\text { day or oral corticosteroids }\end{array}$ & 1 & 209 & $\begin{array}{l}\text { Peto Odds Ratio } \\
\text { (Peto, Fixed, 95\% CI) }\end{array}$ & $1.75[0.99,3.11]$ \\
\hline $\begin{array}{l}14 \text { Proportion of participants } \\
\text { with serious asthma } \\
\text { exacerbations in relation to } \\
\text { baseline PEF (\% predicted) }\end{array}$ & 1 & 911 & $\begin{array}{l}\text { Peto Odds Ratio } \\
\text { (Peto, Fixed, 95\% CI) }\end{array}$ & $0.97[0.70,1.35]$ \\
\hline 14.1 $\mathrm{PEF}>80 \%$ predicted & 1 & 531 & $\begin{array}{l}\text { Peto Odds Ratio } \\
\text { (Peto, Fixed, 95\% CI) }\end{array}$ & $1.00[0.62,1.61]$ \\
\hline $\begin{array}{l}14.2 \mathrm{PEF} 60 \% \text { to } 80 \% \\
\text { predicted }\end{array}$ & 1 & 210 & $\begin{array}{l}\text { Peto Odds Ratio } \\
\text { (Peto, Fixed, 95\% CI) }\end{array}$ & $0.59[0.32,1.10]$ \\
\hline $14.3 \mathrm{PEF}<60 \%$ predicted & 1 & 170 & $\begin{array}{l}\text { Peto Odds Ratio } \\
\text { (Peto, Fixed, 95\% CI) }\end{array}$ & $1.70[0.85,3.38]$ \\
\hline $\begin{array}{l}15 \text { Respiratory related deaths } \\
\text { or life-threatening events } \\
\text { (SMART) }\end{array}$ & 1 & & $\begin{array}{l}\text { Odds Ratio (M-H, } \\
\text { Fixed, } 95 \% \text { CI) }\end{array}$ & Totals not selected \\
\hline 15.1 Salmeterol vs placebo & 1 & & $\begin{array}{l}\text { Odds Ratio }(\mathrm{M}-\mathrm{H}, \\
\text { Fixed, } 95 \% \mathrm{CI})\end{array}$ & $0.0[0.0,0.0]$ \\
\hline $\begin{array}{l}16 \text { Respiratory related deaths } \\
\text { or life-threatening events } \\
\text { (SMART subgrouped by race) }\end{array}$ & 1 & 23327 & $\begin{array}{l}\text { Odds Ratio (IV, } \\
\text { Fixed, } 95 \% \text { CI) }\end{array}$ & $1.40[0.88,2.21]$ \\
\hline 16.1 Caucasians & 1 & 18642 & $\begin{array}{l}\text { Odds Ratio (IV, } \\
\text { Fixed, } 95 \% \text { CI) }\end{array}$ & $1.04[0.62,1.76]$ \\
\hline 16.2 African Americans & 1 & 4685 & $\begin{array}{l}\text { Odds Ratio (IV, } \\
\text { Fixed, } 95 \% \text { CI) }\end{array}$ & $3.95[1.48,10.53]$ \\
\hline $\begin{array}{l}17 \text { Respiratory related deaths } \\
\text { or life-threatening events } \\
\text { (SMART subgrouped by } \\
\text { baseline ICS use) }\end{array}$ & 1 & 26355 & $\begin{array}{l}\text { Peto Odds Ratio } \\
\text { (Peto, Fixed, 95\% CI) }\end{array}$ & $1.39[0.91,2.12]$ \\
\hline 17.1 ICS at baseline & 1 & 12265 & $\begin{array}{l}\text { Peto Odds Ratio } \\
\text { (Peto, Fixed, 95\% CI) }\end{array}$ & $1.21[0.66,2.22]$ \\
\hline 17.2 No ICS at baseline & 1 & 14090 & $\begin{array}{l}\text { Peto Odds Ratio } \\
\text { (Peto, Fixed, 95\% CI) }\end{array}$ & $1.58[0.87,2.85]$ \\
\hline $\begin{array}{l}18 \text { Respiratory related deaths } \\
\text { or life-threatening events } \\
\text { (SMART subgrouped by race } \\
\text { and ICS (Caucasians)) }\end{array}$ & 1 & 18642 & $\begin{array}{l}\text { Odds Ratio (IV, } \\
\text { Fixed, 95\% CI) }\end{array}$ & $1.04[0.62,1.76]$ \\
\hline 18.1 ICS at baseline & 1 & 9223 & $\begin{array}{l}\text { Odds Ratio (IV, } \\
\text { Fixed, } 95 \% \text { CI) }\end{array}$ & $0.88[0.42,1.84]$ \\
\hline
\end{tabular}




\begin{tabular}{|c|c|c|c|c|}
\hline Outcome or subgroup title & No. of studies & No. of participants & Statistical method & Effect size \\
\hline 18.2 No ICS at baseline & 1 & 9419 & $\begin{array}{l}\text { Odds Ratio (IV, } \\
\text { Fixed, } 95 \% \text { CI) }\end{array}$ & $1.24[0.60,2.58]$ \\
\hline $\begin{array}{l}19 \text { Respiratory related deaths } \\
\text { or life-threatening events } \\
\text { (SMART subgrouped by race } \\
\text { and ICS (African Americans)) }\end{array}$ & 1 & 4685 & $\begin{array}{l}\text { Odds Ratio (IV, } \\
\text { Fixed, 95\% CI) }\end{array}$ & $3.82[1.42,10.28]$ \\
\hline 19.1 ICS at baseline & 1 & 1781 & $\begin{array}{l}\text { Odds Ratio (IV, } \\
\text { Fixed, 95\% CI) }\end{array}$ & $2.92[0.79,10.81]$ \\
\hline 19.2 No ICS at baseline & 1 & 2904 & $\begin{array}{l}\text { Odds Ratio (IV, } \\
\text { Fixed, 95\% CI) }\end{array}$ & $5.47[1.21,24.74]$ \\
\hline $\begin{array}{l}20 \text { Respiratory related deaths } \\
\text { or life-threatening events } \\
\text { (SMART subgrouped by } \\
\text { baseline predicted PEF) }\end{array}$ & 1 & 25714 & $\begin{array}{l}\text { Odds Ratio (IV, } \\
\text { Fixed, 95\% CI) }\end{array}$ & $1.42[0.92,2.20]$ \\
\hline $20.1 \mathrm{PEF}>60 \%$ & 1 & 21026 & $\begin{array}{l}\text { Odds Ratio (IV, } \\
\text { Fixed, } 95 \% \text { CI) }\end{array}$ & $1.04[0.57,1.93]$ \\
\hline $20.2 \mathrm{PEF}=<60 \%$ & 1 & 4688 & $\begin{array}{l}\text { Odds Ratio (IV, } \\
\text { Fixed, } 95 \% \text { CI) }\end{array}$ & $1.96[1.05,3.66]$ \\
\hline $\begin{array}{l}21 \text { Respiratory related deaths } \\
\text { or life-threatening events } \\
\text { (SMART subgrouped by study } \\
\text { phase }\end{array}$ & 1 & 26355 & $\begin{array}{l}\text { Odds Ratio (IV, } \\
\text { Fixed, 95\% CI) }\end{array}$ & $1.39[0.90,2.14]$ \\
\hline 21.1 Phase 1 & 1 & 15342 & $\begin{array}{l}\text { Odds Ratio (IV, } \\
\text { Fixed, } 95 \% \text { CI) }\end{array}$ & $1.46[0.87,2.46]$ \\
\hline 21.2 Phase 2 & 1 & 11013 & $\begin{array}{l}\text { Odds Ratio (IV, } \\
\text { Fixed, } 95 \% \text { CI) }\end{array}$ & $1.25[0.59,2.67]$ \\
\hline $\begin{array}{l}22 \text { All-cause hospitalisation } \\
\text { compared to SAE }\end{array}$ & 1 & & $\begin{array}{l}\text { Peto Odds Ratio } \\
\text { (Peto, Fixed, 95\% CI) }\end{array}$ & Totals not selected \\
\hline $\begin{array}{l}\text { 22.1 Patients with } \\
\text { hospitalisation in SMART }\end{array}$ & 1 & & $\begin{array}{l}\text { Peto Odds Ratio } \\
\text { (Peto, Fixed, 95\% CI) }\end{array}$ & $0.0[0.0,0.0]$ \\
\hline $\begin{array}{l}\text { 22.2 Patients with SAE in } \\
\text { SMART }\end{array}$ & 1 & & $\begin{array}{l}\text { Peto Odds Ratio } \\
\text { (Peto, Fixed, 95\% CI) }\end{array}$ & $0.0[0.0,0.0]$ \\
\hline $\begin{array}{l}23 \text { Adults and children } \\
\text { published non-fatal serious } \\
\text { adverse events }\end{array}$ & 11 & & $\begin{array}{l}\text { Peto Odds Ratio } \\
\text { (Peto, Fixed, 95\% CI) }\end{array}$ & Subtotals only \\
\hline $\begin{array}{l}\text { 23.1 Salmeterol versus } \\
\text { placebo }\end{array}$ & 8 & 2117 & $\begin{array}{l}\text { Peto Odds Ratio } \\
\text { (Peto, Fixed, 95\% CI) }\end{array}$ & $1.13[0.65,1.95]$ \\
\hline $\begin{array}{l}23.2 \text { Salmeterol versus } \\
\text { salbutamol }\end{array}$ & 3 & 26107 & $\begin{array}{l}\text { Peto Odds Ratio } \\
\text { (Peto, Fixed, 95\% CI) }\end{array}$ & $0.99[0.83,1.19]$ \\
\hline $\begin{array}{l}24 \text { Hospitalisations for asthma } \\
\text { (FDA data) }\end{array}$ & 1 & 6043 & $\begin{array}{l}\text { Peto Odds Ratio } \\
\text { (Peto, Fixed, 95\% CI) }\end{array}$ & $2.14[1.16,3.93]$ \\
\hline $\begin{array}{l}\text { 24.1 Salmeterol versus } \\
\text { placebo (FDA US trials asthma } \\
\text { hospitalisations) }\end{array}$ & 1 & 6043 & $\begin{array}{l}\text { Peto Odds Ratio } \\
\text { (Peto, Fixed, 95\% CI) }\end{array}$ & $2.14[1.16,3.93]$ \\
\hline $\begin{array}{l}25 \text { Adults and children all } \\
\text { adverse events }\end{array}$ & 17 & & $\begin{array}{l}\text { Peto Odds Ratio } \\
\text { (Peto, Fixed, 95\% CI) }\end{array}$ & Subtotals only \\
\hline $\begin{array}{l}25.1 \text { Salmeterol versus } \\
\text { placebo }\end{array}$ & 12 & 4017 & $\begin{array}{l}\text { Peto Odds Ratio } \\
\text { (Peto, Fixed, 95\% CI) }\end{array}$ & $1.15[1.00,1.33]$ \\
\hline $\begin{array}{l}25.2 \text { Salmeterol versus } \\
\text { salbutamol }\end{array}$ & 5 & 2130 & $\begin{array}{l}\text { Peto Odds Ratio } \\
\text { (Peto, Fixed, 95\% CI) }\end{array}$ & $0.93[0.77,1.13]$ \\
\hline $\begin{array}{l}26 \text { Adults and children } \\
\text { published adverse events }\end{array}$ & 6 & & $\begin{array}{l}\text { Peto Odds Ratio } \\
\text { (Peto, Fixed, 95\% CI) }\end{array}$ & Subtotals only \\
\hline $\begin{array}{l}26.1 \text { Salmeterol versus } \\
\text { placebo }\end{array}$ & 5 & 1726 & $\begin{array}{l}\text { Peto Odds Ratio } \\
\text { (Peto, Fixed, 95\% CI) }\end{array}$ & $1.07[0.87,1.32]$ \\
\hline $\begin{array}{l}26.2 \text { Salmeterol versus } \\
\text { salbutamol }\end{array}$ & 1 & 667 & $\begin{array}{l}\text { Peto Odds Ratio } \\
\text { (Peto, Fixed, 95\% CI) }\end{array}$ & $0.87[0.63,1.20]$ \\
\hline
\end{tabular}




\begin{tabular}{|c|c|c|c|c|}
\hline Outcome or subgroup title & No. of studies & No. of participants & Statistical method & Effect size \\
\hline $\begin{array}{l}27 \text { Adults and children all } \\
\text { published drug-related adverse } \\
\text { events }\end{array}$ & 11 & & $\begin{array}{l}\text { Peto Odds Ratio } \\
\text { (Peto, Fixed, 95\% CI) }\end{array}$ & Subtotals only \\
\hline $\begin{array}{l}\text { 27.1 Salmeterol versus } \\
\text { placebo }\end{array}$ & 10 & 2755 & $\begin{array}{l}\text { Peto Odds Ratio } \\
\text { (Peto, Fixed, 95\% CI) }\end{array}$ & $1.38[1.04,1.85]$ \\
\hline $\begin{array}{l}\text { 27.2 Salmeterol versus } \\
\text { salbutamol }\end{array}$ & 4 & 766 & $\begin{array}{l}\text { Peto Odds Ratio } \\
\text { (Peto, Fixed, 95\% CI) }\end{array}$ & $1.04[0.73,1.48]$ \\
\hline $\begin{array}{l}28 \text { Adults and children serious } \\
\text { drug-related adverse events }\end{array}$ & 14 & & $\begin{array}{l}\text { Peto Odds Ratio } \\
\text { (Peto, Fixed, 95\% CI) }\end{array}$ & Subtotals only \\
\hline $\begin{array}{l}\text { 28.1 Salmeterol versus } \\
\text { placebo }\end{array}$ & 10 & 3103 & $\begin{array}{l}\text { Peto Odds Ratio } \\
\text { (Peto, Fixed, 95\% CI) }\end{array}$ & $0.92[0.32,2.65]$ \\
\hline $\begin{array}{l}\text { 28.2 Salmeterol versus } \\
\text { salbutamol }\end{array}$ & 4 & 1463 & $\begin{array}{l}\text { Peto Odds Ratio } \\
\text { (Peto, Fixed, 95\% CI) }\end{array}$ & $0.63[0.13,3.07]$ \\
\hline $\begin{array}{l}29 \text { Adults and children non- } \\
\text { fatal asthma-related serious } \\
\text { adverse events (FDA data } \\
\text { shown) }\end{array}$ & 23 & & $\begin{array}{l}\text { Peto Odds Ratio } \\
\text { (Peto, Fixed, 95\% CI) }\end{array}$ & Subtotals only \\
\hline $\begin{array}{l}\text { 29.1 Salmeterol versus } \\
\text { placebo }\end{array}$ & 17 & 5174 & $\begin{array}{l}\text { Peto Odds Ratio } \\
\text { (Peto, Fixed, 95\% CI) }\end{array}$ & $1.59[1.05,2.41]$ \\
\hline $\begin{array}{l}\text { 29.2 Salmeterol versus } \\
\text { placebo (US trial results) }\end{array}$ & 1 & 9275 & $\begin{array}{l}\text { Peto Odds Ratio } \\
\text { (Peto, Fixed, 95\% CI) }\end{array}$ & $2.07[1.36,3.13]$ \\
\hline $\begin{array}{l}\text { 29.3 Salmeterol versus } \\
\text { salbutamol }\end{array}$ & 6 & 2341 & $\begin{array}{l}\text { Peto Odds Ratio } \\
\text { (Peto, Fixed, } 95 \% \text { CI) }\end{array}$ & $0.99[0.54,1.81]$ \\
\hline
\end{tabular}

\section{Analysis 1.1. Comparison 1 Regular salmeterol versus placebo or regular salbutamol, Outcome 1 All-cause mortality}

Review: Regular treatment with salmeterol for chronic asthma: serious adverse events

Comparison: 1 Regular salmeterol versus placebo or regular salbutamol

Outcome: 1 All-cause mortality 


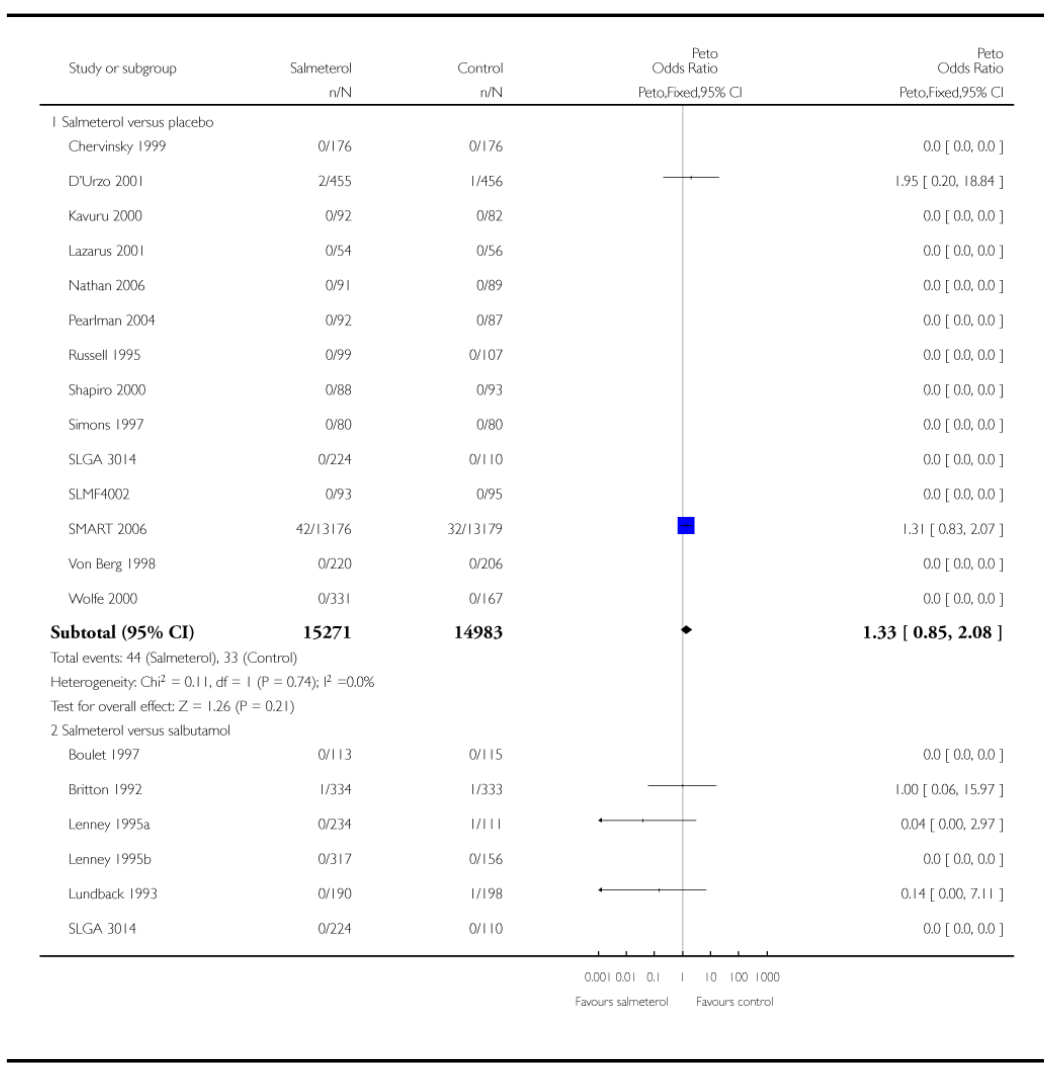

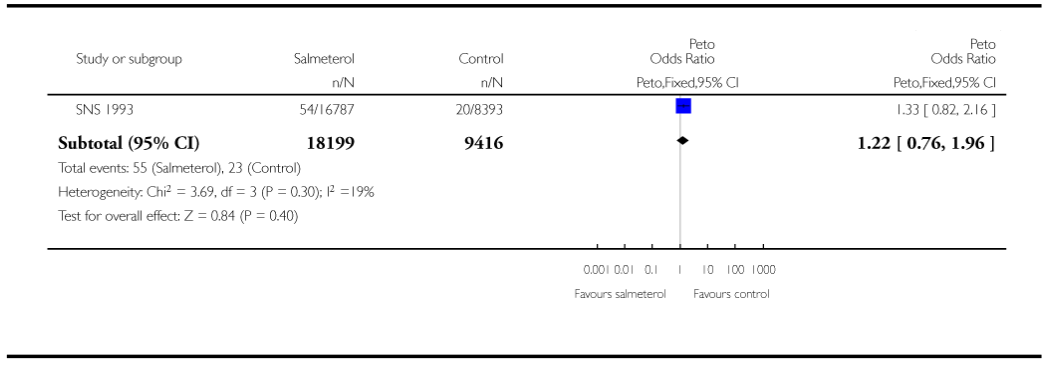

\section{Analysis 1.2. Comparison 1 Regular salmeterol versus placebo or regular salbutamol, Outcome 2 Non-fatal serious adverse events (adults and children)}

Review: Regular treatment with salmeterol for chronic asthma: serious adverse events

Comparison: 1 Regular salmeterol versus placebo or regular salbutamol

Outcome: 2 Non-fatal serious adverse events (adults and children) 


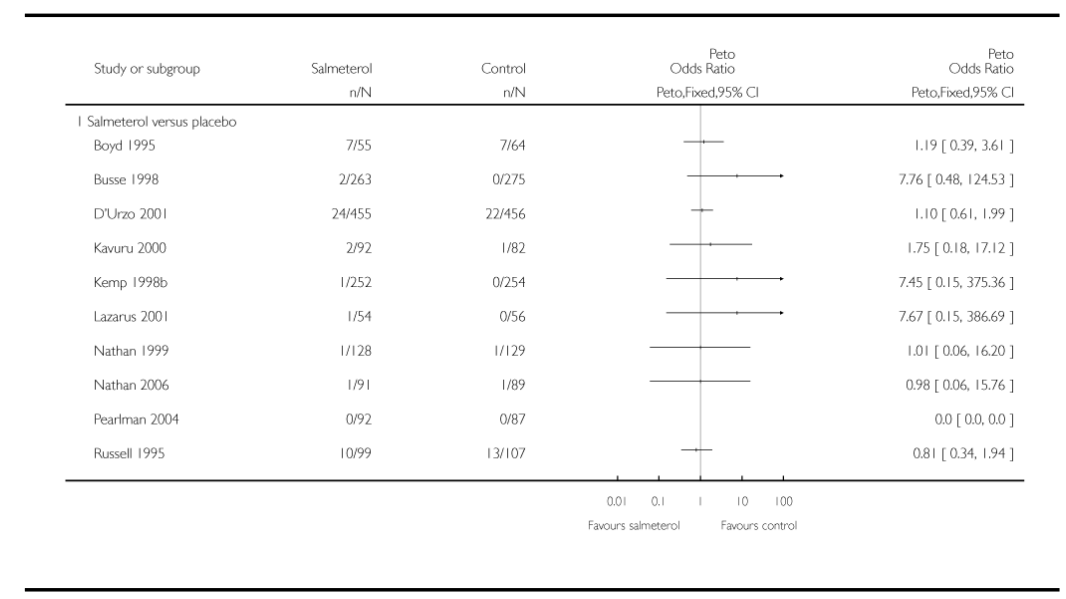

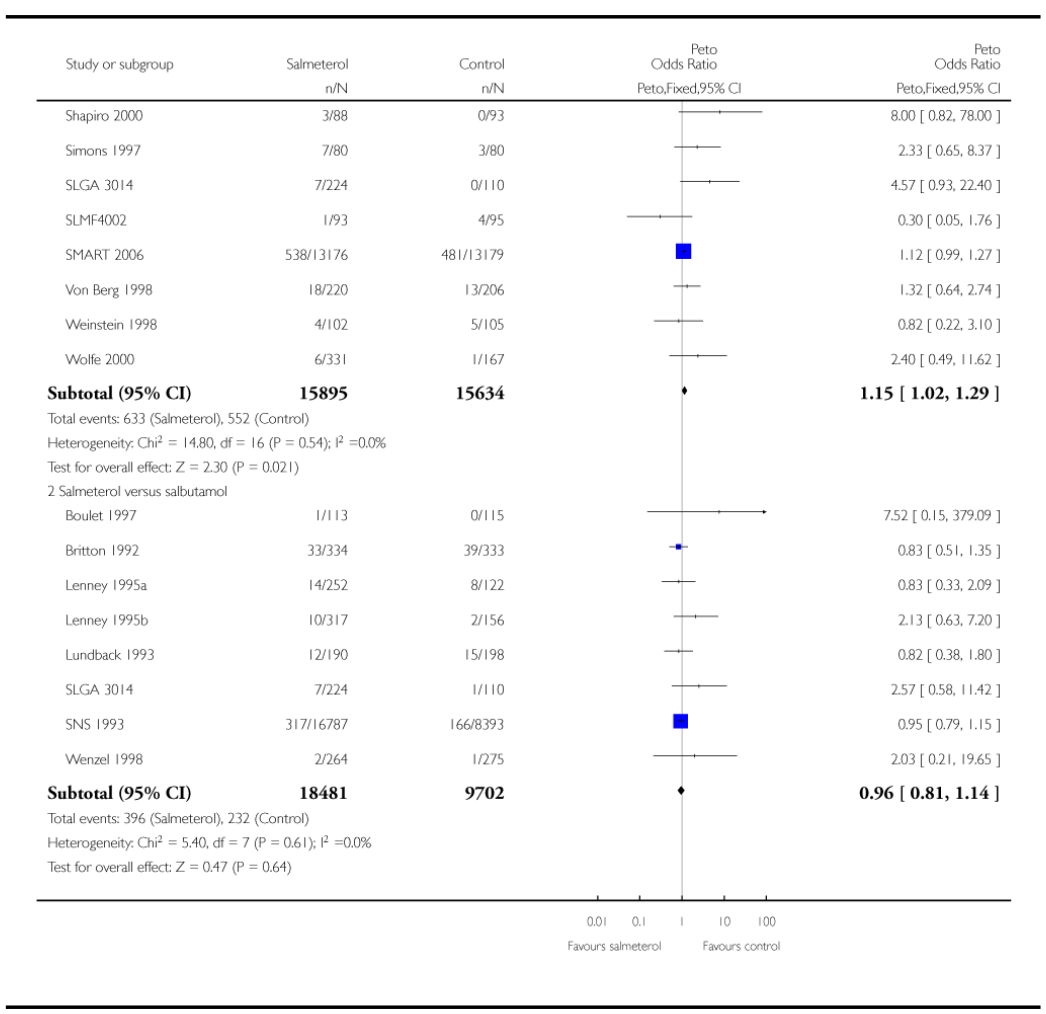

\section{Analysis 1.3. Comparison 1 Regular salmeterol versus placebo or regular salbutamol, Outcome 3 Non-fatal serious adverse events in adults}

Review: Regular treatment with salmeterol for chronic asthma: serious adverse events

Comparison: 1 Regular salmeterol versus placebo or regular salbutamol 
Outcome: 3 Non-fatal serious adverse events in adults

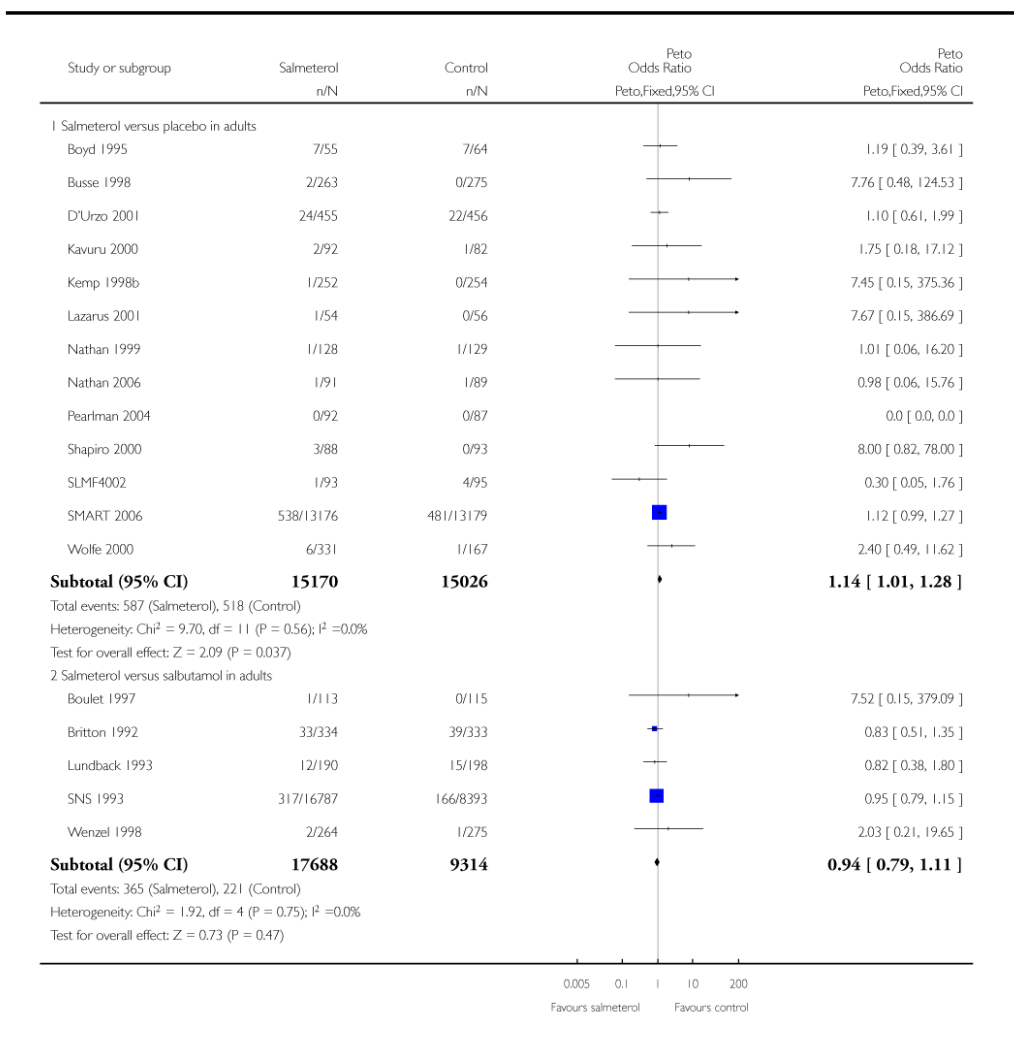

\section{Analysis 1.4. Comparison 1 Regular salmeterol versus placebo or regular salbutamol, Outcome 4 Non-fatal serious adverse events in children}

Review: Regular treatment with salmeterol for chronic asthma: serious adverse events

Comparison: 1 Regular salmeterol versus placebo or regular salbutamol

Outcome: 4 Non-fatal serious adverse events in children 


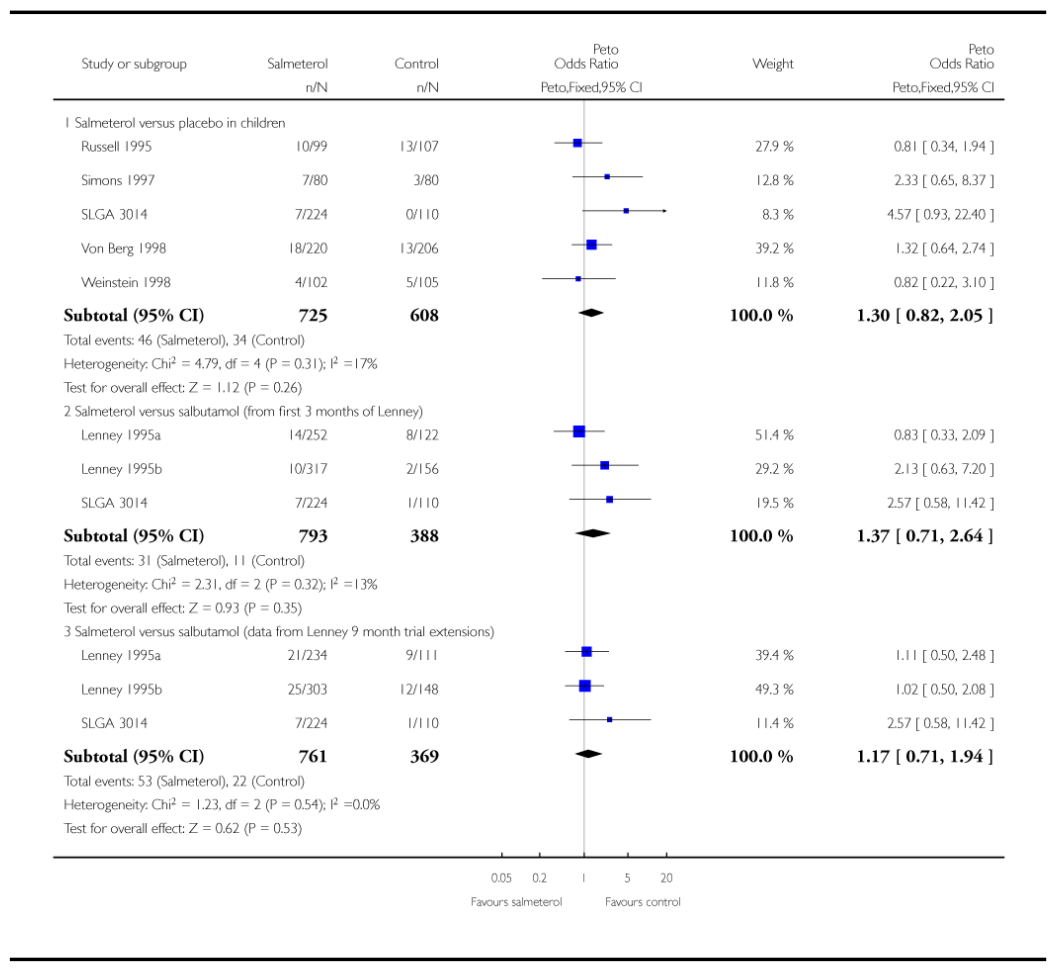

\section{Analysis 1.5. Comparison 1 Regular salmeterol versus placebo or regular salbutamol, Outcome 5 Non-fatal adverse events in adults and children (SNS sensitivity analysis)}

Review: Regular treatment with salmeterol for chronic asthma: serious adverse events

Comparison: 1 Regular salmeterol versus placebo or regular salbutamol

Outcome: 5 Non-fatal adverse events in adults and children (SNS sensitivity analysis) 


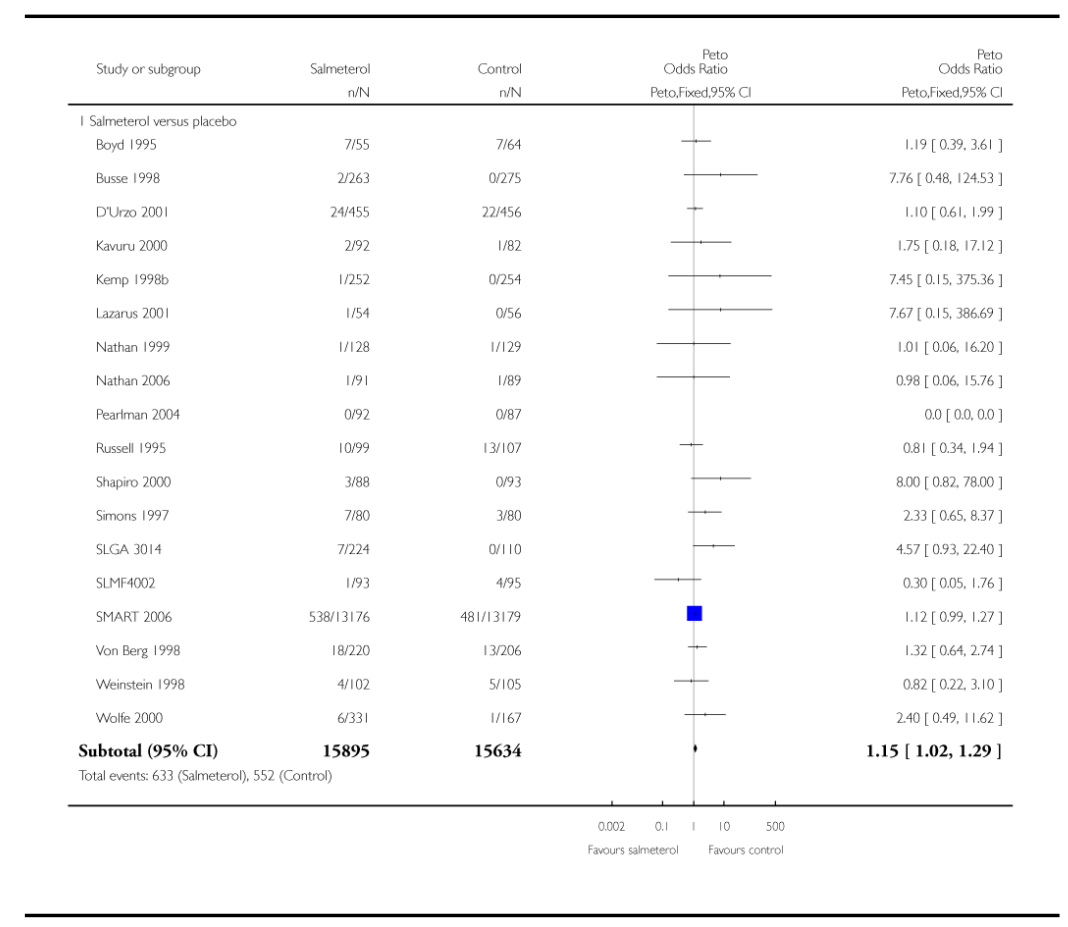

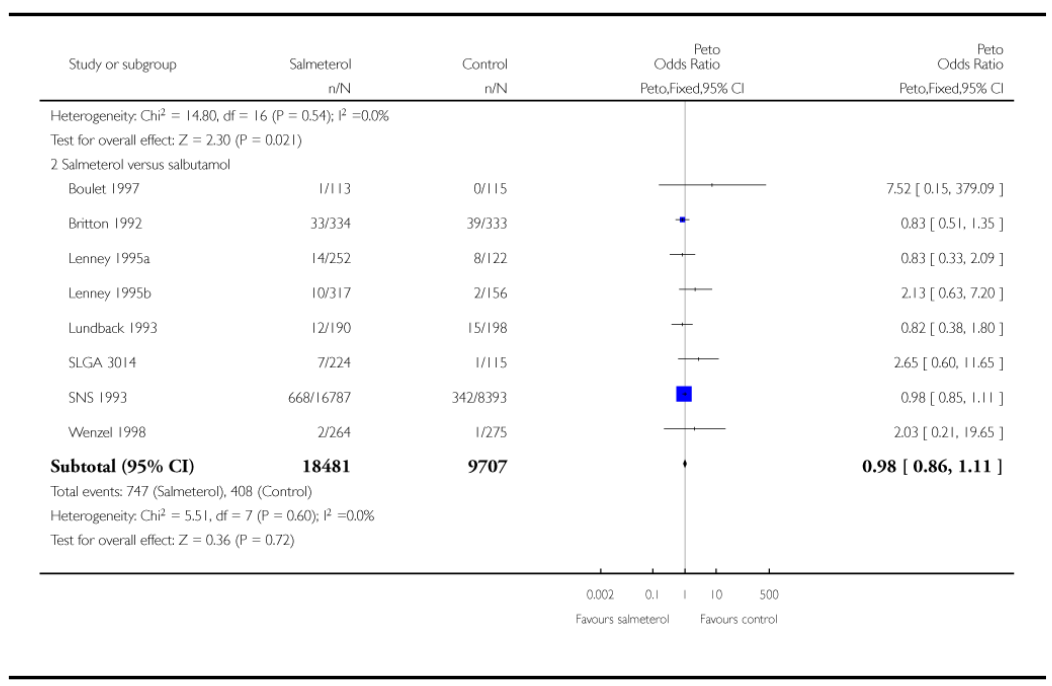

\section{Analysis 1.6. Comparison 1 Regular salmeterol versus placebo or regular salbutamol, Outcome 6 Fatal and non-fatal serious adverse events in adults and children}

Review: Regular treatment with salmeterol for chronic asthma: serious adverse events

Comparison: 1 Regular salmeterol versus placebo or regular salbutamol

Cochrane Database Syst Rev. Author manuscript; available in PMC 2014 May 09. 
Outcome: 6 Fatal and non-fatal serious adverse events in adults and children

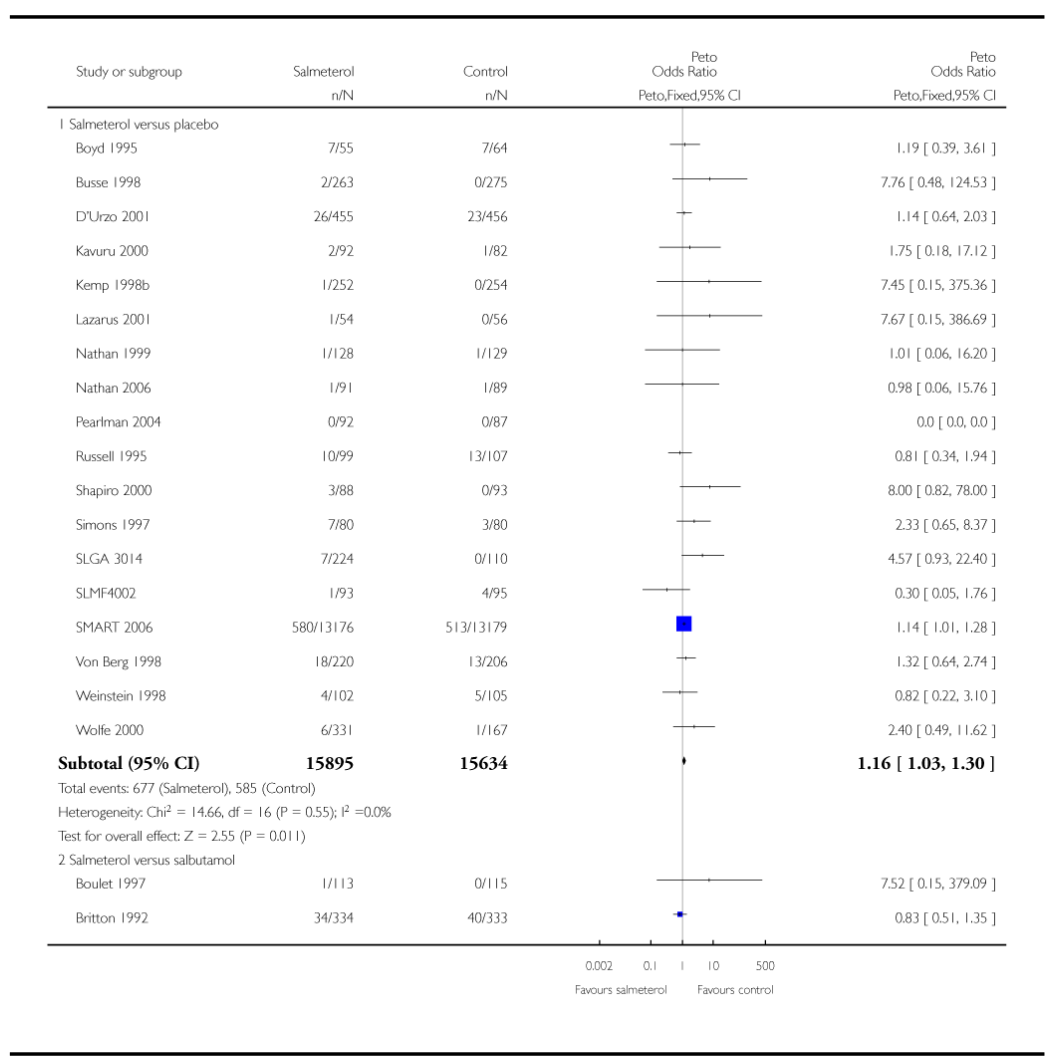

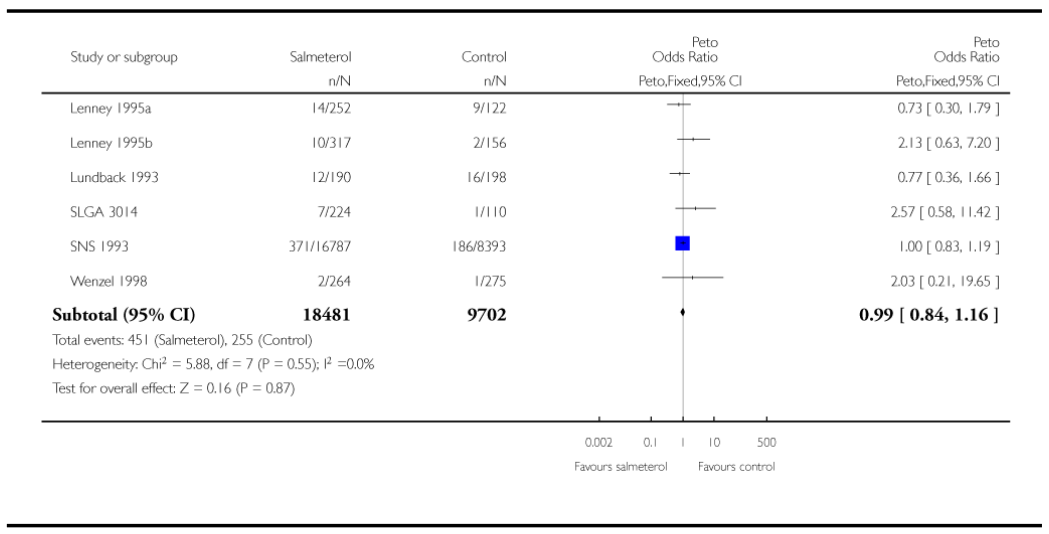

\section{Analysis 1.7. Comparison 1 Regular salmeterol versus placebo or regular salbutamol, Outcome 7 Asthma mortality}

Review: Regular treatment with salmeterol for chronic asthma: serious adverse events

Comparison: 1 Regular salmeterol versus placebo or regular salbutamol 
Outcome: 7 Asthma mortality

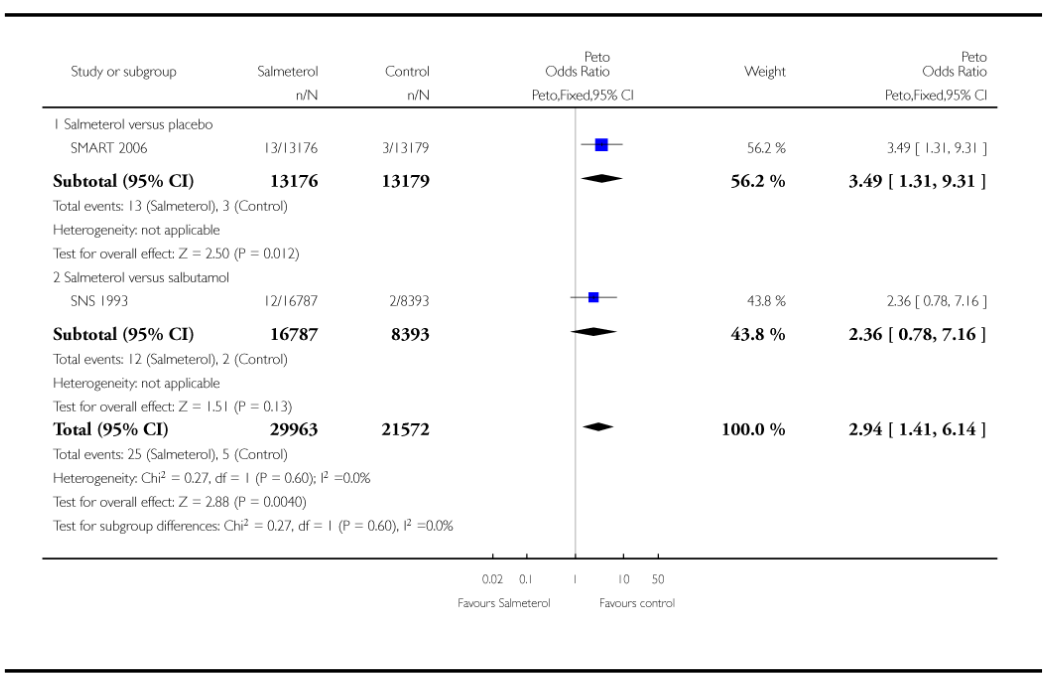

\section{Analysis 1.8. Comparison 1 Regular salmeterol versus placebo or regular salbutamol, Outcome 8 Asthma mortality (within study subgroups by ICS use at baseline)}

Review: Regular treatment with salmeterol for chronic asthma: serious adverse events

Comparison: 1 Regular salmeterol versus placebo or regular salbutamol

Outcome: 8 Asthma mortality (within study subgroups by ICS use at baseline)

\begin{tabular}{|c|c|c|c|c|c|c|}
\hline Study or subgroup & $\begin{array}{r}\text { Salmeterol } \\
n / N\end{array}$ & $\begin{array}{r}\text { Control } \\
\mathrm{n} / \mathrm{N} \\
\end{array}$ & & $\begin{array}{l}\text { Peto } \\
\text { ds antio } \\
\text { ixed,95\% C }\end{array}$ & Weight & $\begin{array}{r}\text { Peto } \\
\text { Odds Ratio } \\
\text { Peto,fixed,95\% Cl } \\
\end{array}$ \\
\hline \multicolumn{7}{|l|}{ I ICS used at baseline } \\
\hline SMART 2006 & $4 / 6127$ & $3 / 6138$ & & $=$ & $25.4 \%$ & $1.33[0.30,5.87]$ \\
\hline SNS 1993 & $7 / 9809$ & 2/4895 & & $=$ & $29.0 \%$ & $1.65[0.41,6.59]$ \\
\hline Subtotal $(95 \% \mathrm{CI})$ & 15936 & 11033 & & - & $54.4 \%$ & $1.49[0.54,4.11]$ \\
\hline \multicolumn{7}{|c|}{ Total events: II (Salmeterol), 5 (Control) } \\
\hline \multicolumn{7}{|c|}{ Heterogeneity: $\mathrm{Ch}^{2}=0.04, \mathrm{df}=\mathrm{I}(\mathrm{P}=0.84) ; \mathrm{I}^{2}=0.0 \%$} \\
\hline \multicolumn{7}{|c|}{ Test for overall effect: $Z=0.77(P=0.44)$} \\
\hline \multicolumn{7}{|l|}{2 Not on ICS at baseline } \\
\hline SMART 2006 & $9 / 7049$ & $0 / 7041$ & & $=$ & $326 \%$ & $7.39[200,27.30]$ \\
\hline SNS 1993 & $4 / 3704$ & 0/1887 & & - & $13.0 \%$ & $4.53[0.57,35.99]$ \\
\hline Subtotal $(95 \% \mathrm{CI})$ & 10753 & 8928 & & $\sim$ & $45.6 \%$ & $6.43[2.13,19.42]$ \\
\hline \multicolumn{7}{|c|}{ Total events: 13 (Salmeterol), 0 (Control) } \\
\hline \multicolumn{7}{|c|}{ Heterogeneity: $\mathrm{Ch}^{2}=0.15, \mathrm{df}=I(P=0.70) ; P^{2}=0.0 \%$} \\
\hline \multicolumn{7}{|c|}{ Test for overall effect: $Z=3.30(P=0.00097)$} \\
\hline Total $(95 \% \mathrm{CI})$ & 26689 & 19961 & & - & $100.0 \%$ & $2.90[1.38,6.13]$ \\
\hline \multicolumn{7}{|c|}{ Total events: 24 (Salmeterol), 5 (Control) } \\
\hline \multicolumn{7}{|c|}{ Heterogeneity: $\mathrm{Chi}^{2}=3.84, \mathrm{df}=3(\mathrm{P}=0.28) ; 1^{2}=22 \%$} \\
\hline \multicolumn{7}{|c|}{ Test for overall effect: $Z=2.80(P=0.0051)$} \\
\hline \multicolumn{7}{|c|}{ Test for subgroup differences: $\mathrm{Chi}^{2}=3.65, \mathrm{df}=1(\mathrm{P}=0.06), \mathrm{I}^{2}=73 \%$} \\
\hline & & & 0.002 & 500 & & \\
\hline & & & Favours sameterol & Farours control & & \\
\hline
\end{tabular}




\section{Analysis 1.9. Comparison 1 Regular salmeterol versus placebo or regular salbutamol, Outcome 9 Asthma mortality (within study subgroups by any steroid at baseline)}

Review: Regular treatment with salmeterol for chronic asthma: serious adverse events

Comparison: 1 Regular salmeterol versus placebo or regular salbutamol

Outcome: 9 Asthma mortality (within study subgroups by any steroid at baseline)

\begin{tabular}{|c|c|c|c|c|c|c|}
\hline Study or subgroup & $\begin{array}{r}\text { Salmeterol } \\
n / N\end{array}$ & $\begin{array}{r}\text { Control } \\
n / N\end{array}$ & $\begin{array}{l}\text { Odds } \\
\text { Peto,fix }\end{array}$ & 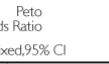 & Weight & $\begin{array}{r}\text { Peto } \\
\text { Odds Ratio } \\
\text { Peto,fixed,95\% Cl }\end{array}$ \\
\hline \multicolumn{7}{|c|}{ I ICS or oral steroids used at baseline } \\
\hline SMART 2006 & $7 / 6127$ & $3 / 6138$ & & $=$ & $35.1 \%$ & $2.23[0.65,7.71]$ \\
\hline SNS 1993 & 8/10409 & 2/5195 & & - & $31.2 \%$ & $1.82[0.49,6.78]$ \\
\hline Subtotal $(95 \% \mathrm{CI})$ & 16536 & 11333 & & $\longrightarrow$ & $66.3 \%$ & $2.03[0.82,5.00]$ \\
\hline \multicolumn{7}{|c|}{ Total events: 15 (Salmeterol), 5 (Control) } \\
\hline \multicolumn{7}{|c|}{ Heterogeneity. $C h i^{2}=0.05, \mathrm{df}=1(\mathrm{P}=0.83) ; 1^{2}=0.0 \%$} \\
\hline \multicolumn{7}{|c|}{ Test for overall effect: $Z=1.53(P=0.12)$} \\
\hline \multicolumn{7}{|c|}{2 Not any steroids at baseline } \\
\hline SMART 2006 & 6/7049 & $0 / 7041$ & & - & $21.1 \%$ & $7.39[1.49,36.60]$ \\
\hline SNS 1993 & 4/3704 & 0/1887 & & $\cdot-$ & $12.6 \%$ & $4.53[0.57,35.99]$ \\
\hline Subtotal $(95 \% \mathrm{CI})$ & 10753 & 8928 & & - & $33.7 \%$ & $6.15[1.73,21.84]$ \\
\hline \multicolumn{7}{|c|}{ Total events: 10 (Salmeterol). 0 (Control) } \\
\hline \multicolumn{7}{|c|}{ Heterogeneity. $\mathrm{Chi}^{2}=0.13, \mathrm{df}=1(\mathrm{P}=0.71): \mathrm{I}^{2}=0.0 \%$} \\
\hline \multicolumn{7}{|c|}{ Test for overall effect. $Z=2.81(P=0.0049)$} \\
\hline Total $(95 \% \mathrm{CI})$ & 27289 & 20261 & & - & $100.0 \%$ & $2.95[1.41,6.14]$ \\
\hline \multicolumn{7}{|c|}{ Total events: 25 (Salmeterol), 5 (Control) } \\
\hline \multicolumn{7}{|c|}{ Heterogeneity: $\mathrm{Chi}^{2}=2.14, \mathrm{df}=3(\mathrm{P}=0.54): 1^{2}=0.0 \%$} \\
\hline \multicolumn{7}{|c|}{ Test for overall effect: $Z=2.88(P=0.0040)$} \\
\hline \multicolumn{7}{|c|}{ Test for subgroup differences: $C h^{2}=1.96, \mathrm{df}=1(P=0.16), F^{2}=49 \%$} \\
\hline & & & 0.002 & $1 \quad 10 \quad 500$ & & \\
\hline & & & Favours salmeterol & Favours control & & \\
\hline
\end{tabular}

\section{Analysis 1.10. Comparison 1 Regular salmeterol versus placebo or regular salbutamol, Outcome 10 Cardiovascular mortality}

Review: Regular treatment with salmeterol for chronic asthma: serious adverse events

Comparison: 1 Regular salmeterol versus placebo or regular salbutamol

Outcome: 10 Cardiovascular mortality 


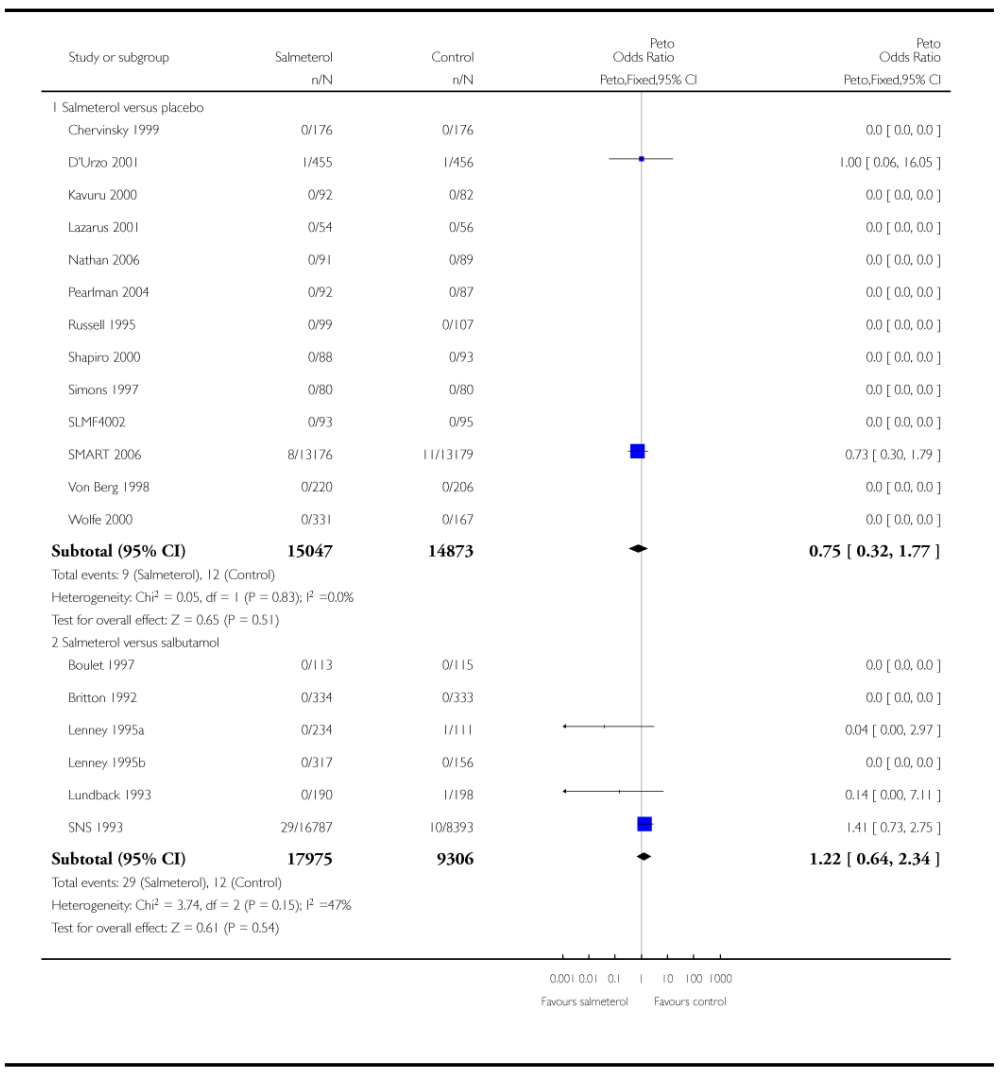

\section{Analysis 1.11. Comparison 1 Regular salmeterol versus placebo or regular salbutamol, Outcome 11 Adults and Children non-fatal asthma-related serious adverse events}

Review: Regular treatment with salmeterol for chronic asthma: serious adverse events

Comparison: 1 Regular salmeterol versus placebo or regular salbutamol

Outcome: 11 Adults and Children non-fatal asthma-related serious adverse events 


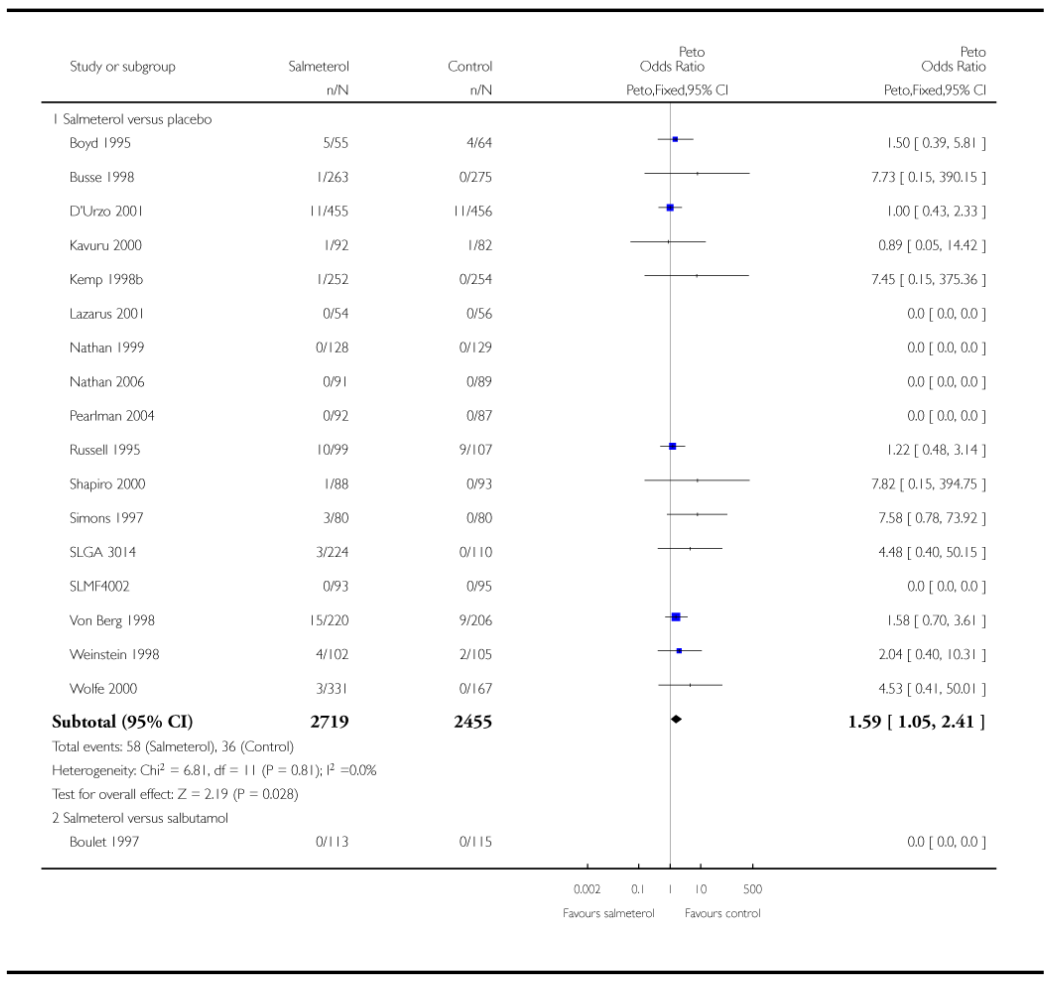

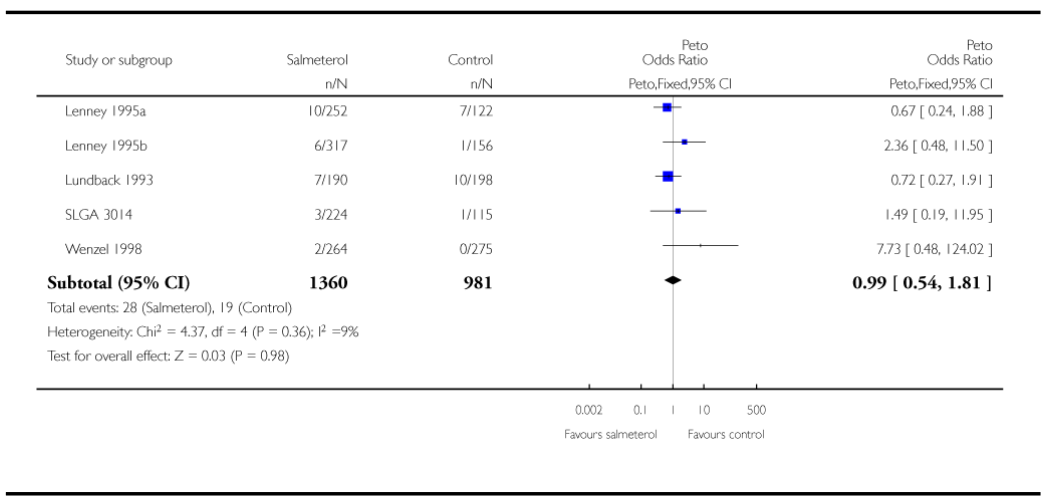

\section{Analysis 1.12. Comparison 1 Regular salmeterol versus placebo or regular salbutamol, Outcome 12 Adults and children non-fatal cardiovascular serious adverse events}

Review: Regular treatment with salmeterol for chronic asthma: serious adverse events

Comparison: 1 Regular salmeterol versus placebo or regular salbutamol

Outcome: 12 Adults and children non-fatal cardiovascular serious adverse events 


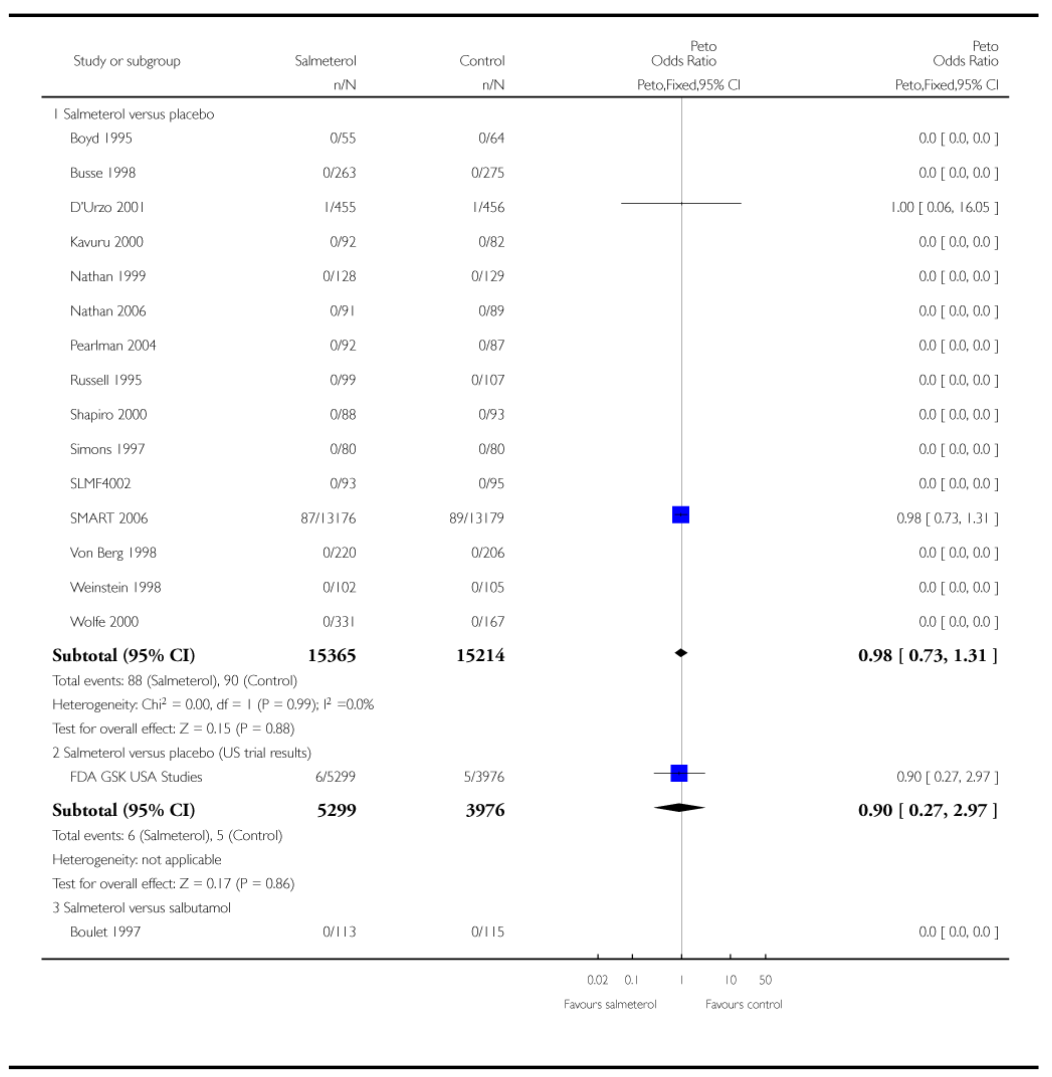

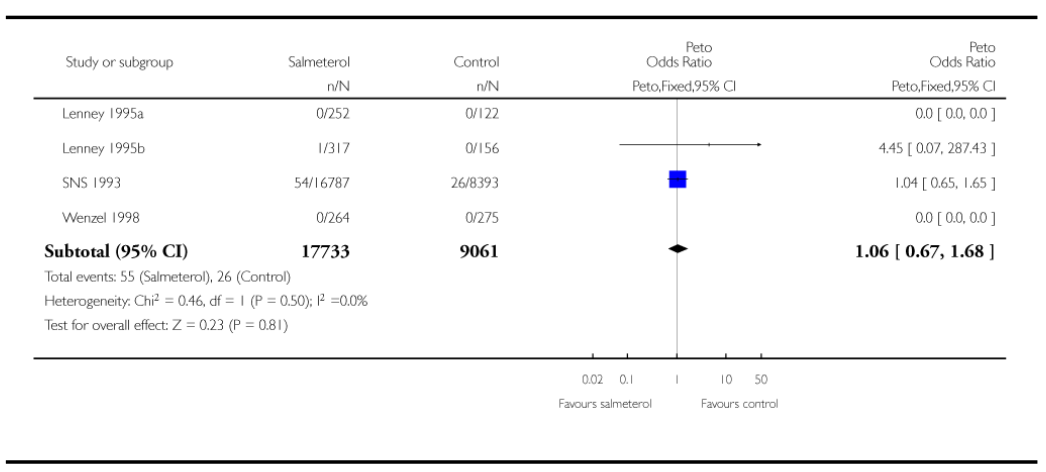

\section{Analysis 1.13. Comparison 1 Regular salmeterol versus placebo or regular salbutamol, Outcome 13 Proportion of participants with serious asthma exacerbations in relation to dose of ICS}

Review: Regular treatment with salmeterol for chronic asthma: serious adverse events

Comparison: 1 Regular salmeterol versus placebo or regular salbutamol 
Outcome: 13 Proportion of participants with serious asthma exacerbations in relation to dose of ICS

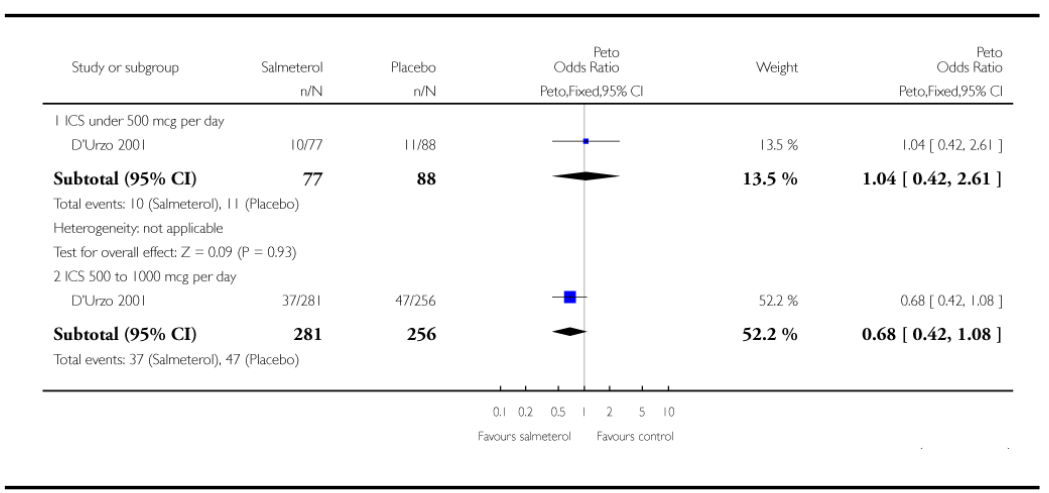

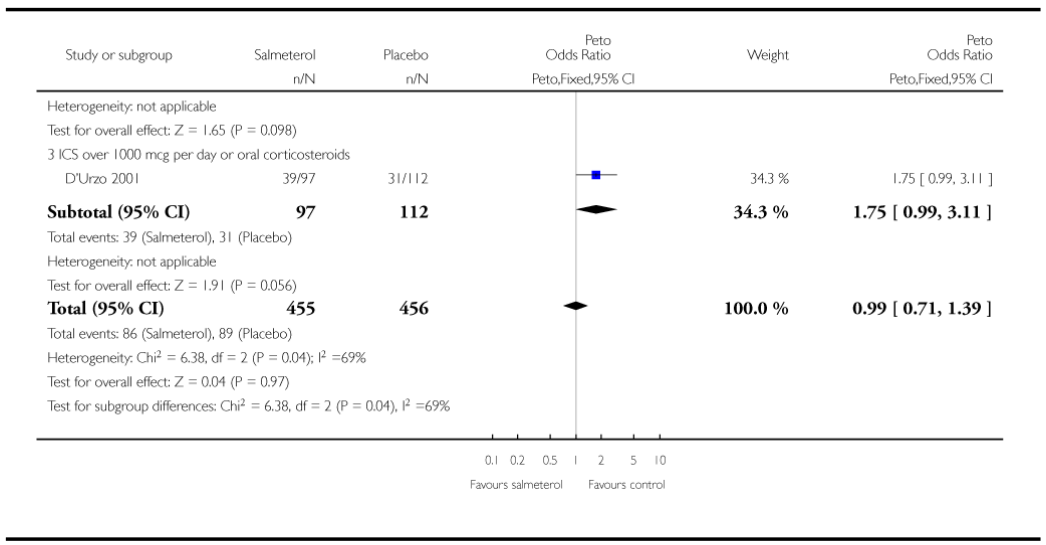

\section{Analysis 1.14. Comparison 1 Regular salmeterol versus placebo or regular salbutamol, Outcome 14 Proportion of participants with serious asthma exacerbations in relation to baseline PEF (\% predicted)}

Review: Regular treatment with salmeterol for chronic asthma: serious adverse events

Comparison: 1 Regular salmeterol versus placebo or regular salbutamol

Outcome: 14 Proportion of participants with serious asthma exacerbations in relation to baseline PEF (\% predicted) 


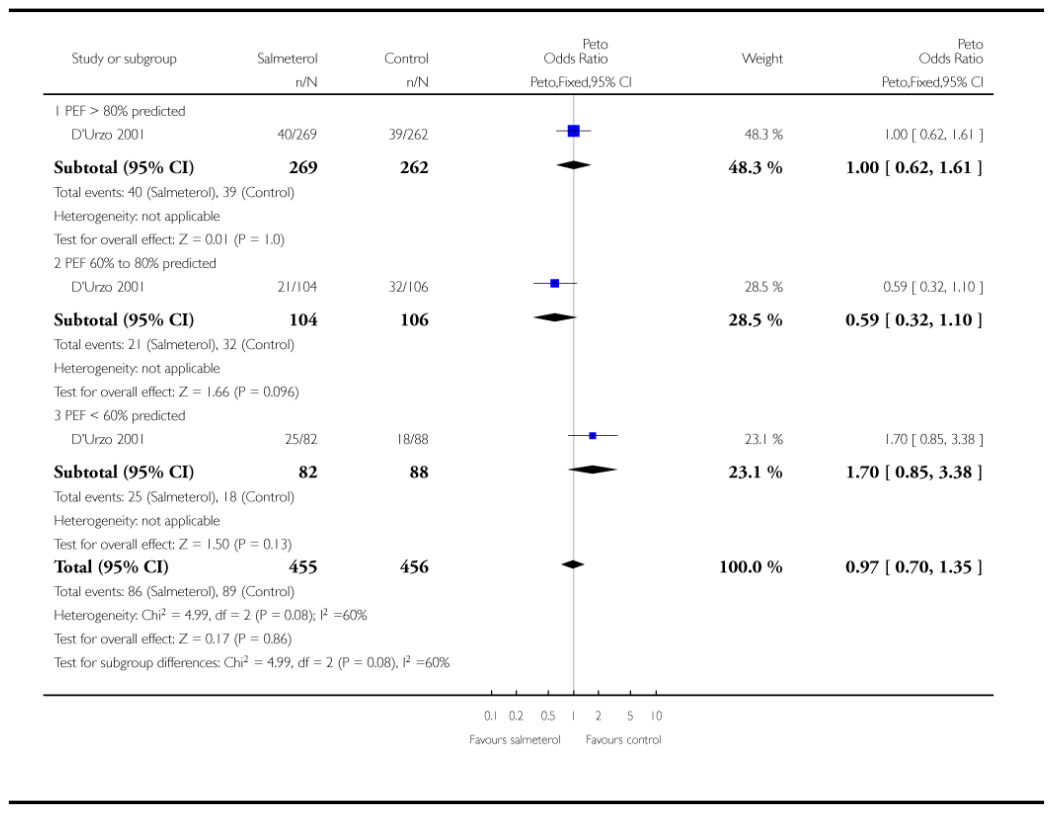

\section{Analysis 1.15. Comparison 1 Regular salmeterol versus placebo or regular salbutamol, Outcome 15 Respiratory related deaths or life-threatening events (SMART)}

Review: Regular treatment with salmeterol for chronic asthma: serious adverse events

Comparison: 1 Regular salmeterol versus placebo or regular salbutamol

Outcome: 15 Respiratory related deaths or life-threatening events (SMART)

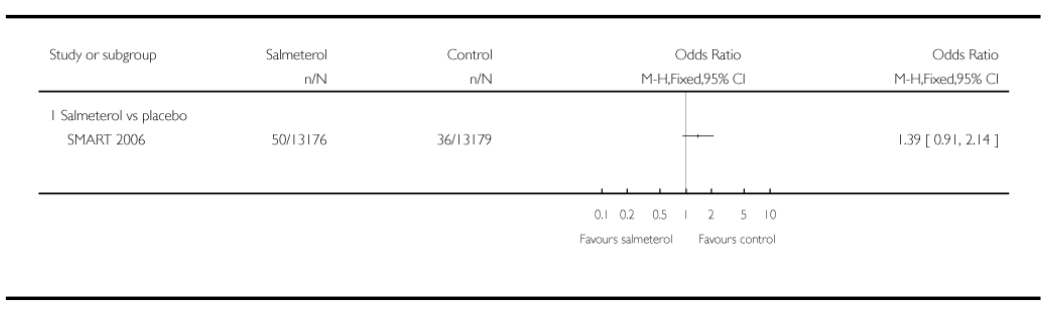

\section{Analysis 1.16. Comparison 1 Regular salmeterol versus placebo or regular salbutamol, Outcome 16 Respiratory related deaths or life-threatening events (SMART subgrouped by race)}

Review: Regular treatment with salmeterol for chronic asthma: serious adverse events

Comparison: 1 Regular salmeterol versus placebo or regular salbutamol0 
Outcome: 16 Respiratory related deaths or life-threatening events (SMART subgrouped by race)

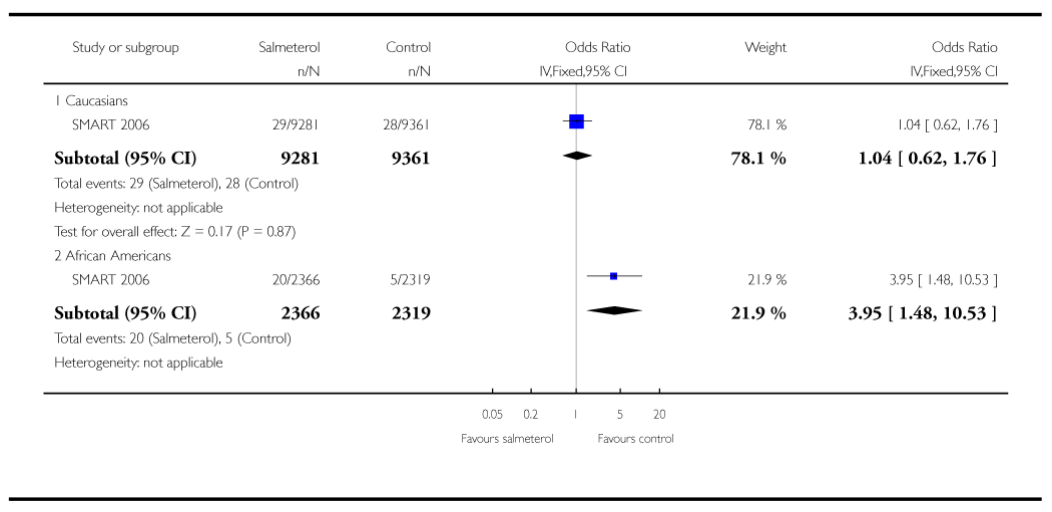

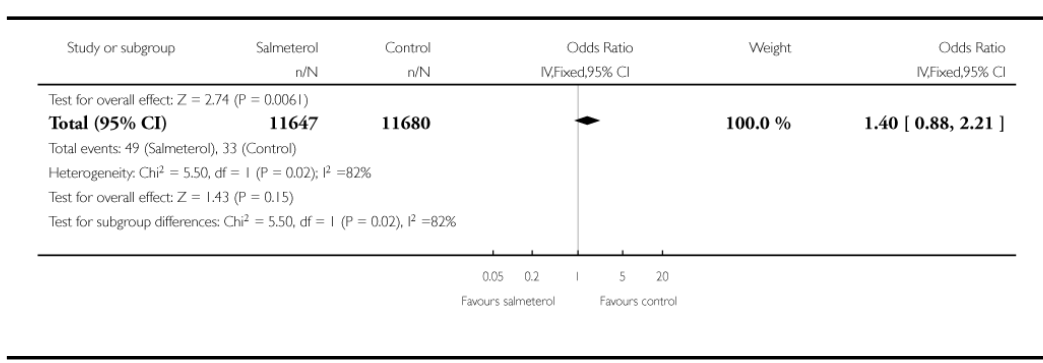

\section{Analysis 1.17. Comparison 1 Regular salmeterol versus placebo or regular salbutamol, Outcome 17 Respiratory related deaths or life-threatening events (SMART subgrouped by baseline ICS use)}

Review: Regular treatment with salmeterol for chronic asthma: serious adverse events

Comparison: 1 Regular salmeterol versus placebo or regular salbutamol

Outcome: 17 Respiratory related deaths or life-threatening events (SMART subgrouped by baseline ICS use) 


\begin{tabular}{|c|c|c|c|c|c|}
\hline Study or subgroup & $\begin{array}{r}\text { Salmeterol } \\
\mathrm{n} / \mathrm{N}\end{array}$ & $\begin{array}{r}\text { Control } \\
\mathrm{n} N \mathrm{~N}\end{array}$ & $\begin{array}{c}\text { Peto } \\
\text { Odds Ratio } \\
\text { Peto,fixed,95\% CI }\end{array}$ & Weight & $\begin{array}{r}\text { Peto } \\
\text { Ods Ratio } \\
\text { Peto,fixed,95\% CI }\end{array}$ \\
\hline $\begin{array}{l}\text { 1 ICS at baseline } \\
\text { SMART } 2006\end{array}$ & $23 / 6127$ & $19 / 6138$ & $=$ & $48.8 \%$ & $1.21[0.66,2.22]$ \\
\hline \multicolumn{6}{|c|}{$\begin{array}{l}\text { Total events: } 23 \text { (SSalmeteror). } 19 \text { (Control) } \\
\text { Heterogeneity. not appicable } \\
\text { Test for overal leffect: } Z=0.62 \text { (P = }=0.53) \\
2 \text { No ICS at baseline }\end{array}$} \\
\hline 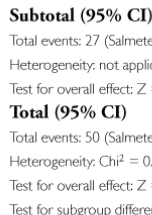 & 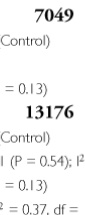 & $\begin{array}{l}13179 \\
0.54), p^{2}=0.0 \%\end{array}$ & - & $100.0 \%$ & $1.58[0.87,2.85]$ \\
\hline & & & \begin{tabular}{c|c}
0.5 & 2 \\
neterol & Favours
\end{tabular} & & \\
\hline
\end{tabular}

\section{Analysis 1.18. Comparison 1 Regular salmeterol versus placebo or regular salbutamol, Outcome 18 Respiratory related deaths or life-threatening events (SMART subgrouped by race and ICS (Caucasians))}

Review: Regular treatment with salmeterol for chronic asthma: serious adverse events

Comparison: 1 Regular salmeterol versus placebo or regular salbutamol

Outcome: 18 Respiratory related deaths or life-threatening events (SMART subgrouped by race and ICS (Caucasians))

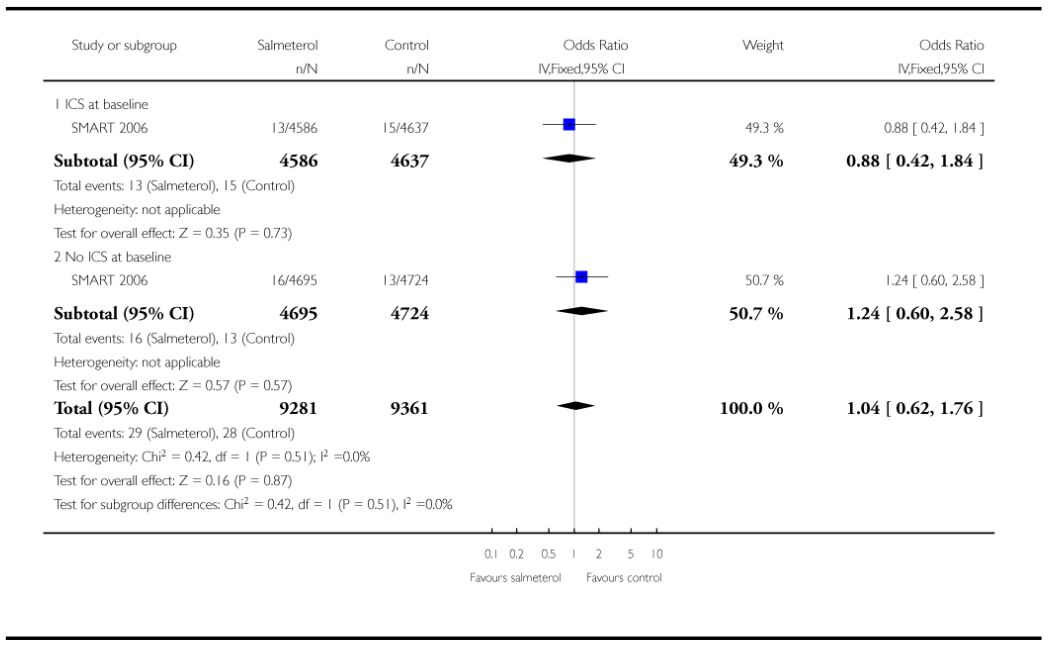




\section{Analysis 1.19. Comparison 1 Regular salmeterol versus placebo or regular salbutamol, Outcome 19 Respiratory related deaths or life-threatening events (SMART subgrouped by race and ICS (African Americans))}

Review: Regular treatment with salmeterol for chronic asthma: serious adverse events

Comparison: 1 Regular salmeterol versus placebo or regular salbutamol

Outcome: 19 Respiratory related deaths or life-threatening events (SMART subgrouped by race and ICS (African Americans))

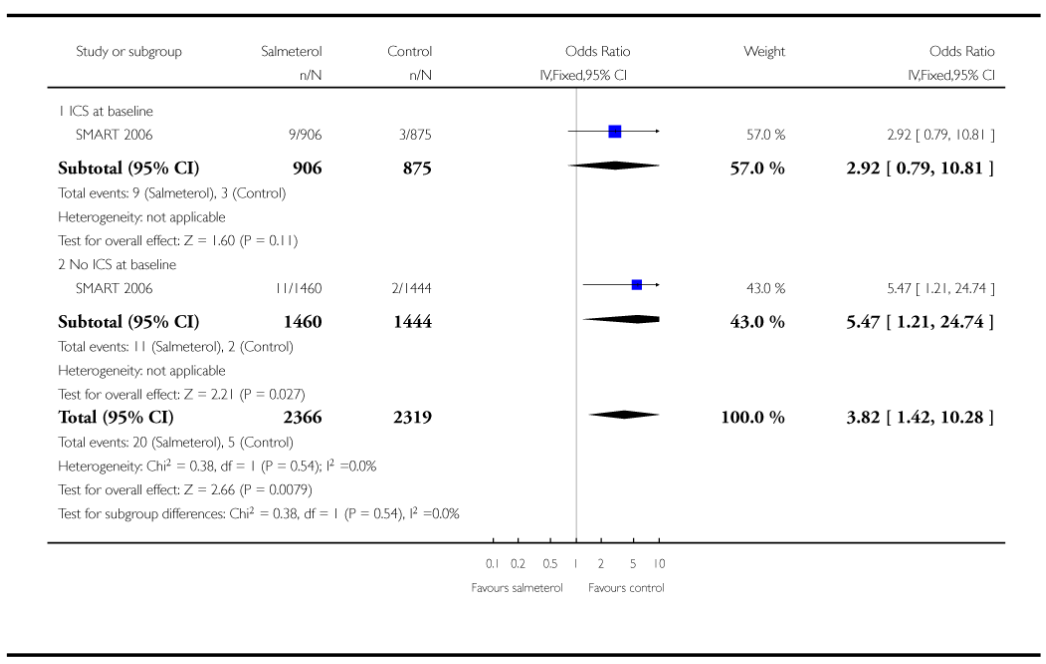

\section{Analysis 1.20. Comparison 1 Regular salmeterol versus placebo or regular salbutamol, Outcome 20 Respiratory related deaths or life-threatening events (SMART subgrouped by baseline predicted PEF)}

Review: Regular treatment with salmeterol for chronic asthma: serious adverse events

Comparison: 1 Regular salmeterol versus placebo or regular salbutamol

Outcome: 20 Respiratory related deaths or life-threatening events (SMART subgrouped by baseline predicted PEF) 


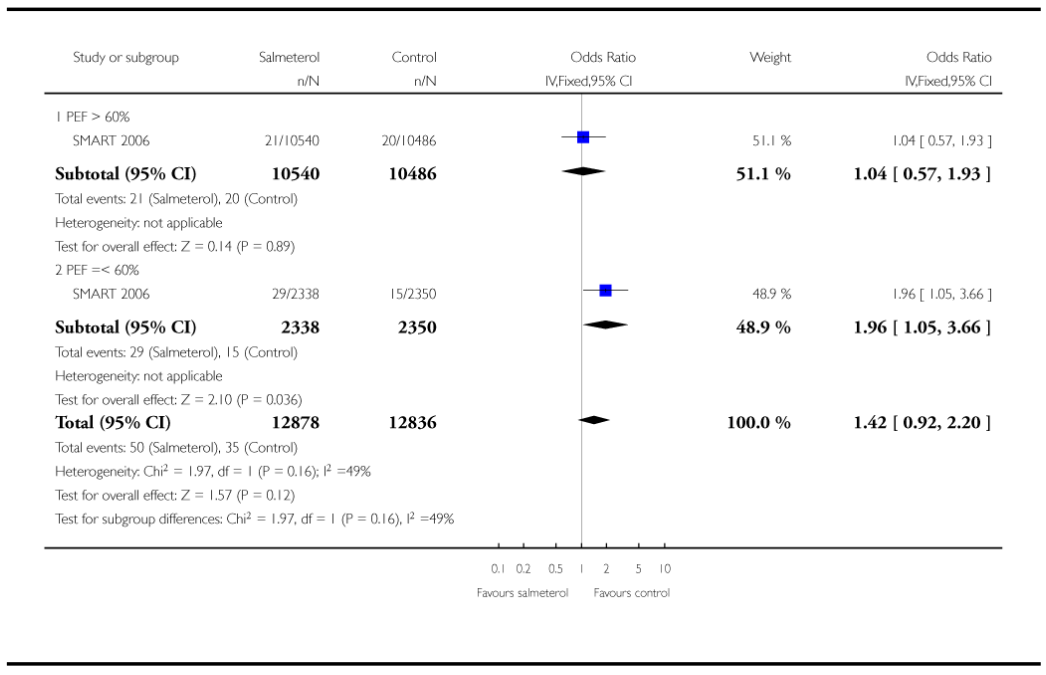

\section{Analysis 1.21. Comparison 1 Regular salmeterol versus placebo or regular salbutamol, Outcome 21 Respiratory related deaths or life-threatening events (SMART subgrouped by study phase}

Review: Regular treatment with salmeterol for chronic asthma: serious adverse events

Comparison: 1 Regular salmeterol versus placebo or regular salbutamol

Outcome: 21 Respiratory related deaths or life-threatening events (SMART subgrouped by study phase

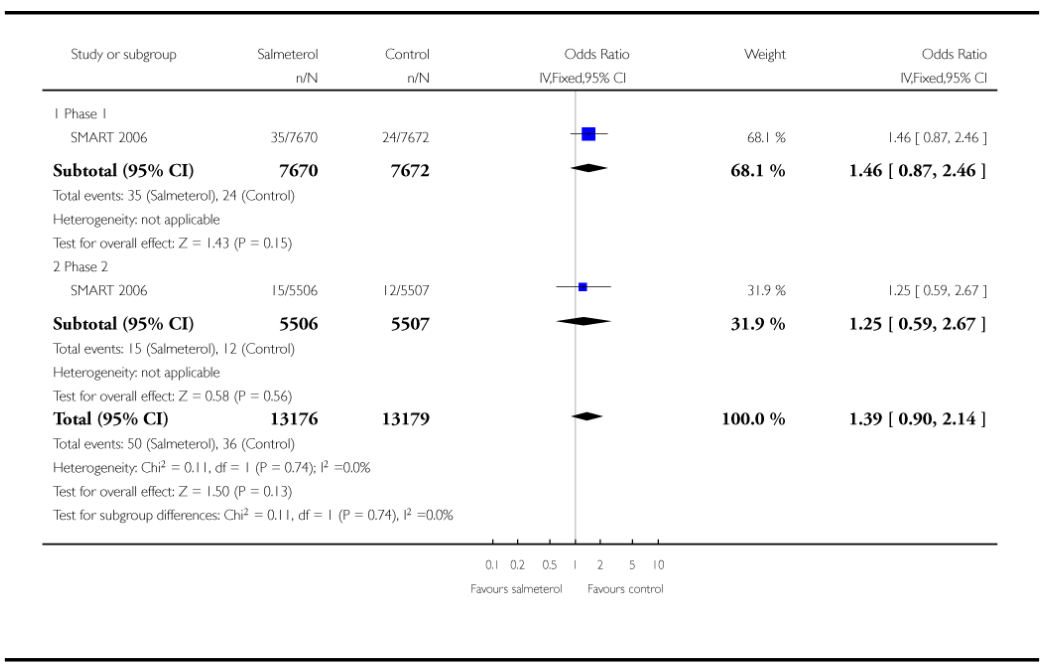




\section{Analysis 1.22. Comparison 1 Regular salmeterol versus placebo or regular salbutamol, Outcome 22 All-cause hospitalisation compared to SAE}

Review: Regular treatment with salmeterol for chronic asthma: serious adverse events

Comparison: 1 Regular salmeterol versus placebo or regular salbutamol

Outcome: 22 All-cause hospitalisation compared to SAE

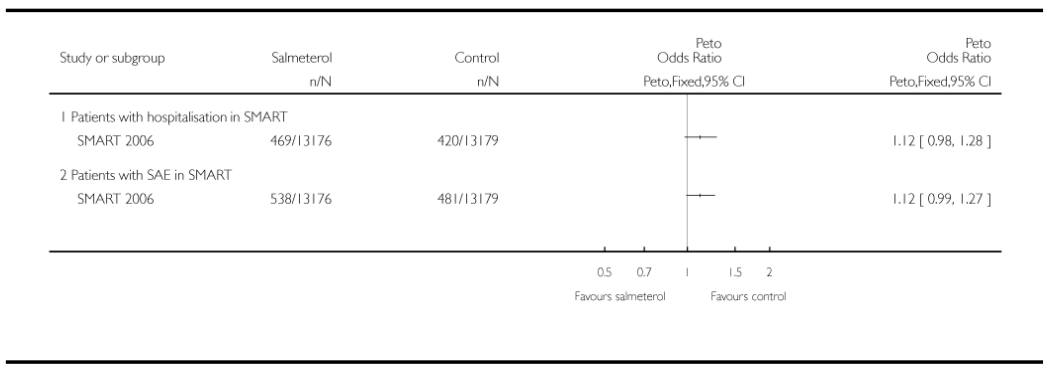

\section{Analysis 1.23. Comparison 1 Regular salmeterol versus placebo or regular salbutamol, Outcome 23 Adults and children published non-fatal serious adverse events}

Review: Regular treatment with salmeterol for chronic asthma: serious adverse events

Comparison: 1 Regular salmeterol versus placebo or regular salbutamol

Outcome: 23 Adults and children published non-fatal serious adverse events 


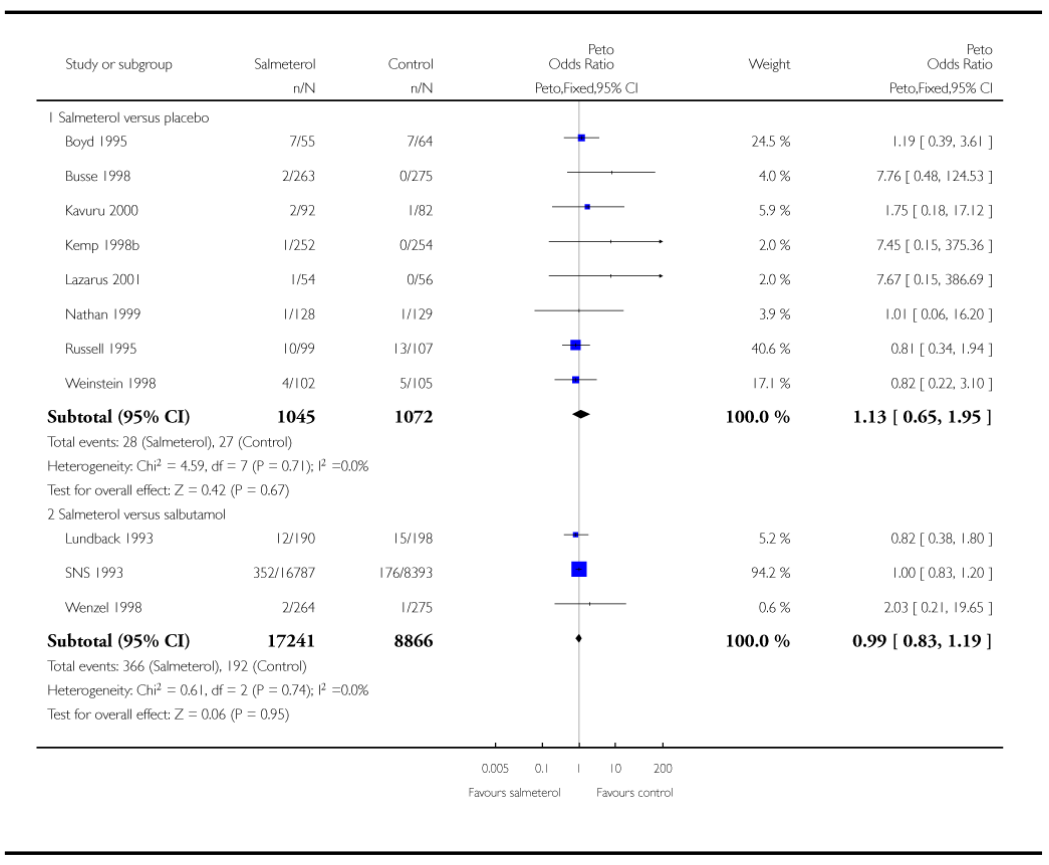

Analysis 1.24. Comparison 1 Regular salmeterol versus placebo or regular salbutamol, Outcome 24 Hospitalisations for asthma (FDA data)

Review: Regular treatment with salmeterol for chronic asthma: serious adverse events

Comparison: 1 Regular salmeterol versus placebo or regular salbutamol

Outcome: 24 Hospitalisations for asthma (FDA data)

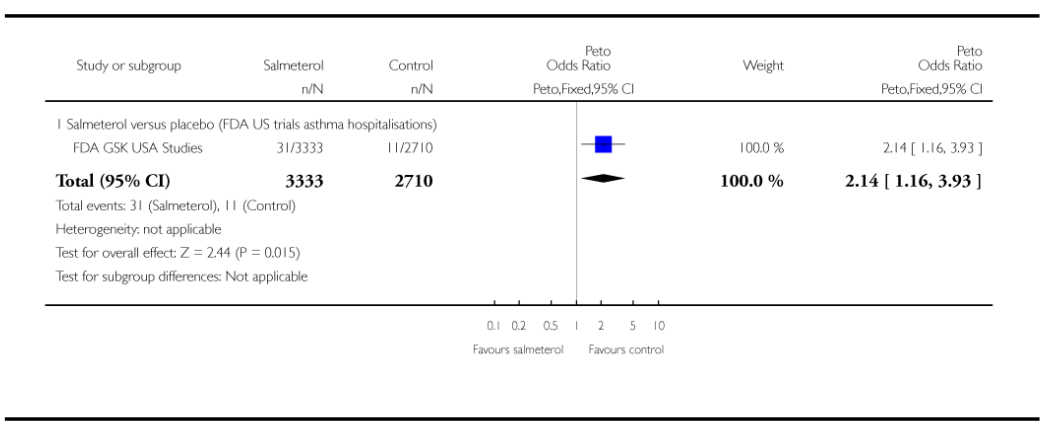

Analysis 1.25. Comparison 1 Regular salmeterol versus placebo or regular salbutamol, Outcome 25 Adults and children all adverse events

Review: Regular treatment with salmeterol for chronic asthma: serious adverse events 
Comparison: 1 Regular salmeterol versus placebo or regular salbutamol

Outcome: 25 Adults and children all adverse events

\begin{tabular}{|c|c|c|c|c|c|}
\hline Study or subgroup & $\begin{array}{r}\text { Salmeterol } \\
\mathrm{n} / \mathrm{N}\end{array}$ & $\begin{array}{r}\text { Control } \\
\mathrm{n} / \mathrm{N}\end{array}$ & $\begin{aligned} & \text { Peto } \\
& \text { Oddd Ratio } \\
& \text { Peto, Fixed,95\% Cl }\end{aligned}$ & Weight & $\begin{array}{r}\text { Peto } \\
\text { Odds RRatio } \\
\text { Peto,Fixed,95\% Cl }\end{array}$ \\
\hline \multicolumn{6}{|l|}{ I Salmeterol versus placebo } \\
\hline D'Urzo 2001 & 39/455 & $36 / 456$ & $=$ & $9.5 \%$ & $1.09[0.68,1.75]$ \\
\hline Kavuru 2000 & $57 / 92$ & $38 / 82$ & - & $6.0 \%$ & $1.87[1.03,3.40]$ \\
\hline Kemp 1998b & 134/252 & $130 / 254$ & - & $17.5 \%$ & $1.08[0.76,1.53]$ \\
\hline Nathan 2006 & 60191 & $53 / 89$ & $=$ & $5.9 \%$ & $1.31[0.72,2.40]$ \\
\hline Pearlman 2004 & $55 / 92$ & $49 / 87$ & 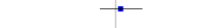 & $6.1 \%$ & $1.15[0.64,2.08]$ \\
\hline Rosenthal 1999 & 149/202 & 151/206 & - & $11.0 \%$ & $1.02[0.66,1.59]$ \\
\hline Russell 1995 & 74/99 & $81 / 107$ & $\rightarrow$ & $5.3 \%$ & $0.95[0.51,1.79]$ \\
\hline Shapiro 2000 & $51 / 88$ & 48/93 & - & $6.2 \%$ & $1.29[0.72,2.31]$ \\
\hline Simons 1997 & 78/80 & $74 / 80$ & 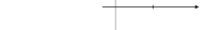 & $1.1 \%$ & $2.85[0.69,11.75]$ \\
\hline SLMF4002 & 34/93 & 41/95 & $\rightarrow$ & $6.3 \%$ & $0.76[0.42,1.36]$ \\
\hline Von Berg 1998 & 170/220 & $157 / 206$ & - & $10.5 \%$ & $1.06[0.68,1.66]$ \\
\hline Wolfe 2000 & $213 / 331$ & $96 / 167$ & - & $14.5 \%$ & $1.34[0.91,1.96]$ \\
\hline Subtotal $(95 \% \mathrm{CI})$ & 2095 & 1922 & - & $100.0 \%$ & $1.15[1.00,1.33]$ \\
\hline \multirow{2}{*}{\multicolumn{6}{|c|}{$\begin{array}{l}\text { Total events: } 1114 \text { (Salmeterol), } 954 \text { (Control) } \\
\text { Heterogeneity: Chi' }=7.90, \text { df }=11(P=0.72) ; 1^{2}=0.0 \%\end{array}$}} \\
\hline & & & & & \\
\hline \multicolumn{6}{|c|}{ Test for overall effect: $Z=1.92(P=0.055)$} \\
\hline \multicolumn{6}{|c|}{2 Salmeterol versus salbutamol } \\
\hline Boulet 1997 & $89 / 113$ & $82 / 115$ & $=$ & $10.2 \%$ & $1.49[0.82,2.70]$ \\
\hline Britton 1992 & $107 / 334$ & $117 / 333$ & $=$ & $35.4 \%$ & $0.87[0.63,1.20]$ \\
\hline Lenney 1995a & $165 / 252$ & $81 / 122$ & - & $17.6 \%$ & $0.96[0.61,1.51]$ \\
\hline Lenney 1995b & $202 / 317$ & $99 / 156$ & $\rightarrow$ & $23.0 \%$ & $1.01[0.68,1.51]$ \\
\hline Lundback 1993 & $29 / 190$ & $42 / 198$ & $\rightarrow$ & $13.8 \%$ & $0.67[0.40,1.12]$ \\
\hline Subtotal $(95 \% \mathrm{CI})$ & 1206 & 924 & - & $100.0 \%$ & $0.93[0.77,1.13]$ \\
\hline \multirow{3}{*}{\multicolumn{6}{|c|}{$\begin{array}{l}\text { Total events: } 592 \text { (Salmeterol), } 421 \text { (Control) } \\
\text { Heterogeneity: Chi' }=4.23, d f=4(P=0.38) ;\left.\right|^{2}=6 \% \\
\text { Test for overall effect: } Z=0.70(P=0.48)\end{array}$}} \\
\hline & & & & & \\
\hline & & & & & \\
\hline & & & 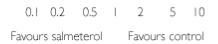 & & \\
\hline
\end{tabular}

\section{Analysis 1.26. Comparison 1 Regular salmeterol versus placebo or regular salbutamol, Outcome 26 Adults and children published adverse events}

Review: Regular treatment with salmeterol for chronic asthma: serious adverse events

Comparison: 1 Regular salmeterol versus placebo or regular salbutamol

Outcome: 26 Adults and children published adverse events 


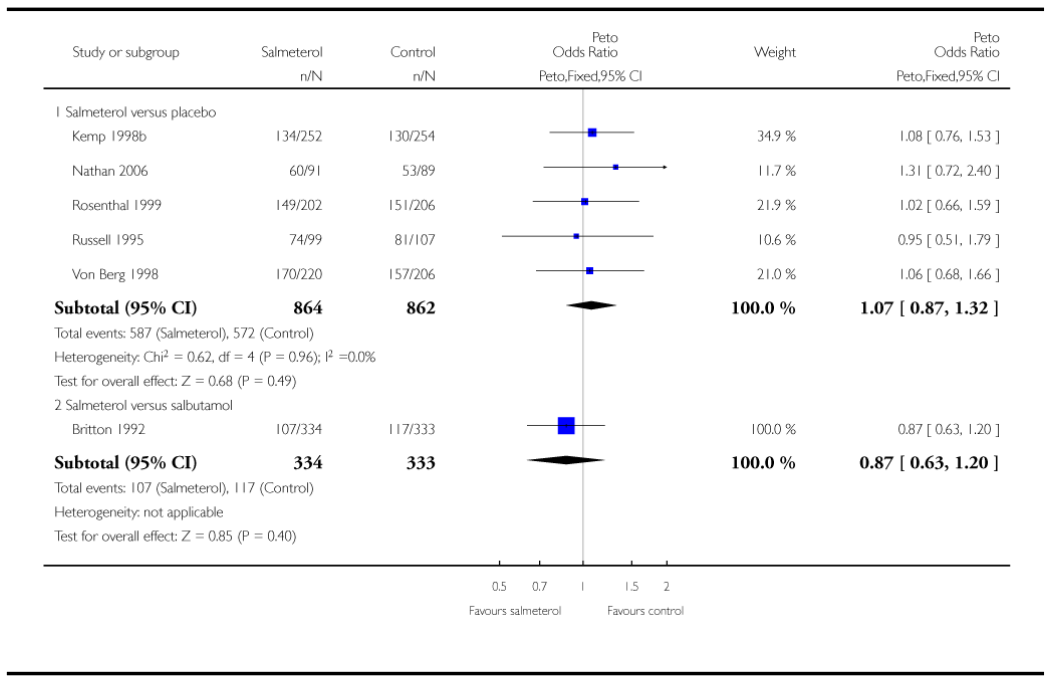

\section{Analysis 1.27. Comparison 1 Regular salmeterol versus placebo or regular salbutamol, Outcome 27 Adults and children all published drug-related adverse events}

Review: Regular treatment with salmeterol for chronic asthma: serious adverse events

Comparison: 1 Regular salmeterol versus placebo or regular salbutamol

Outcome: 27 Adults and children all published drug-related adverse events 


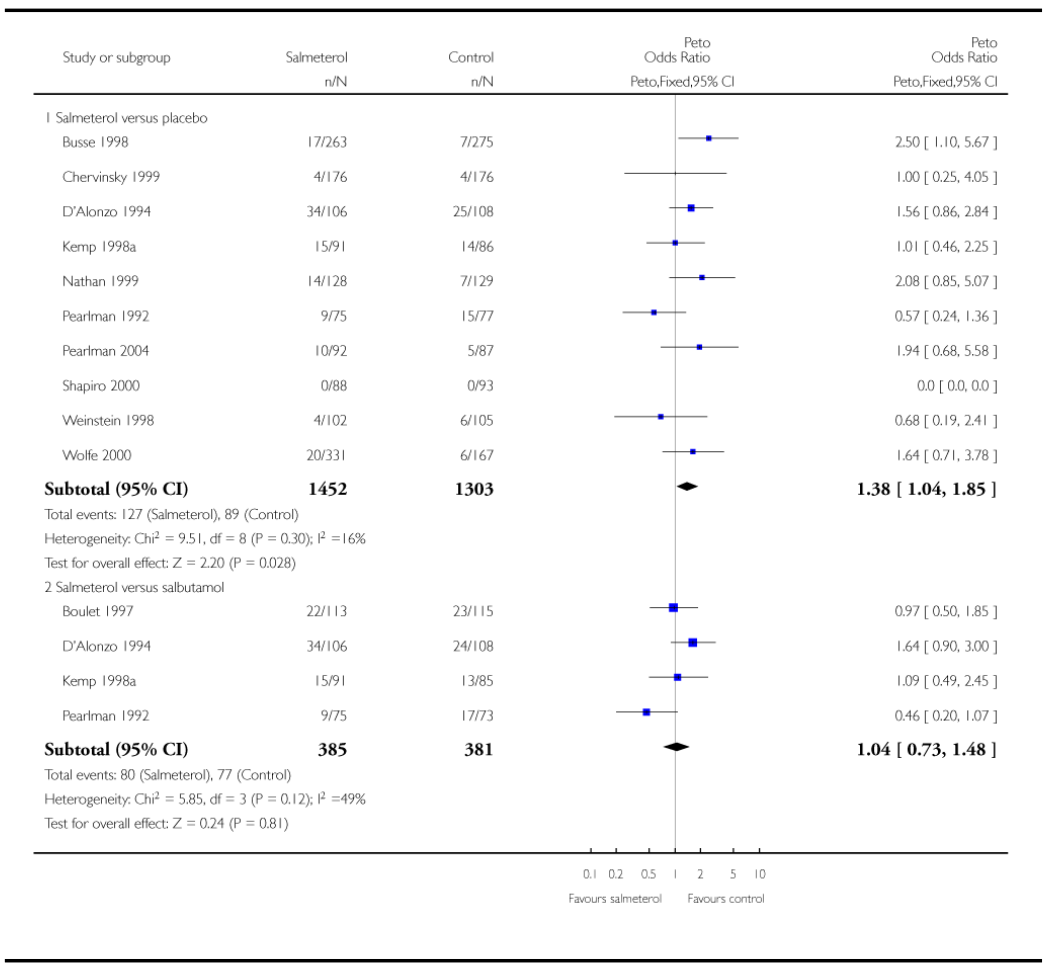

\section{Analysis 1.28. Comparison 1 Regular salmeterol versus placebo or regular salbutamol, Outcome 28 Adults and children serious drug-related adverse events}

Review: Regular treatment with salmeterol for chronic asthma: serious adverse events

Comparison: 1 Regular salmeterol versus placebo or regular salbutamol

Outcome: 28 Adults and children serious drug-related adverse events 


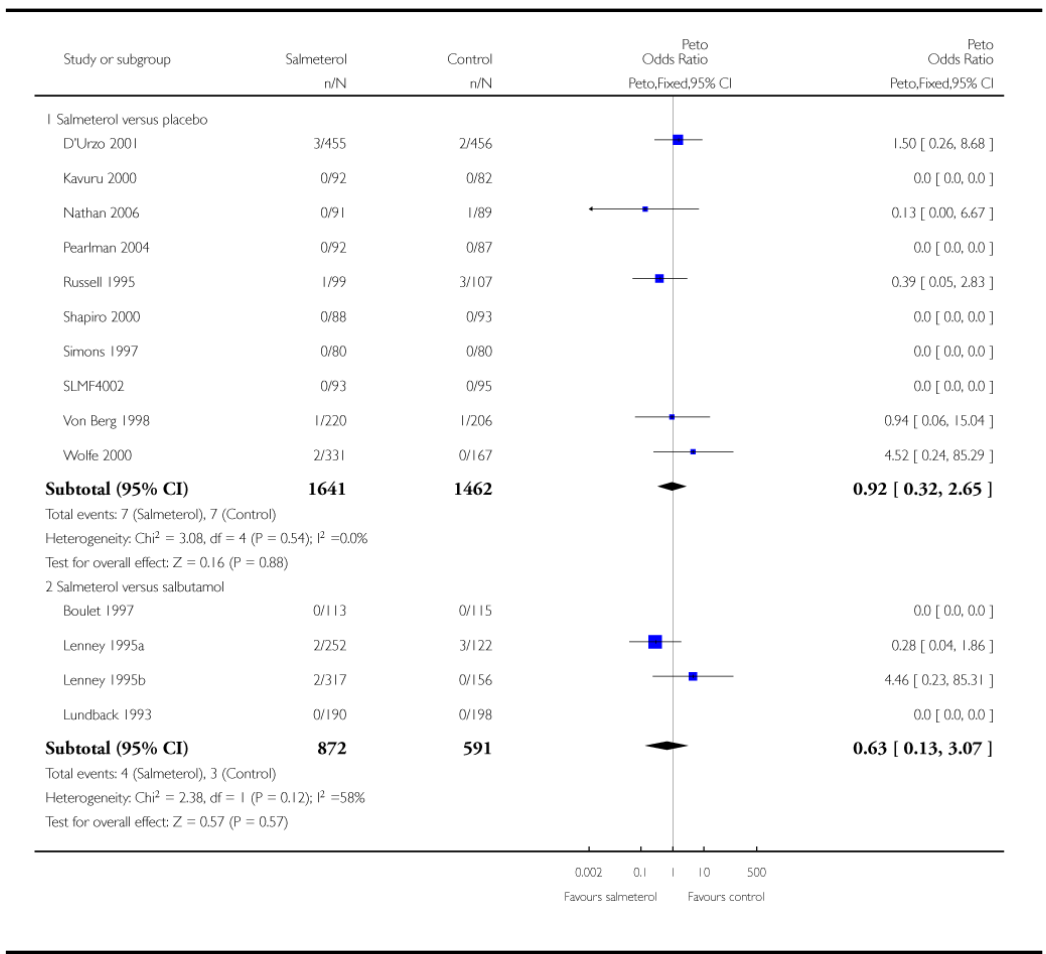

\section{Analysis 1.29. Comparison 1 Regular salmeterol versus placebo or regular salbutamol, Outcome 29 Adults and children non-fatal asthma-related serious adverse events (FDA data shown)}

Review: Regular treatment with salmeterol for chronic asthma: serious adverse events

Comparison: 1 Regular salmeterol versus placebo or regular salbutamol

Outcome: 29 Adults and children non-fatal asthma-related serious adverse events (FDA data shown) 


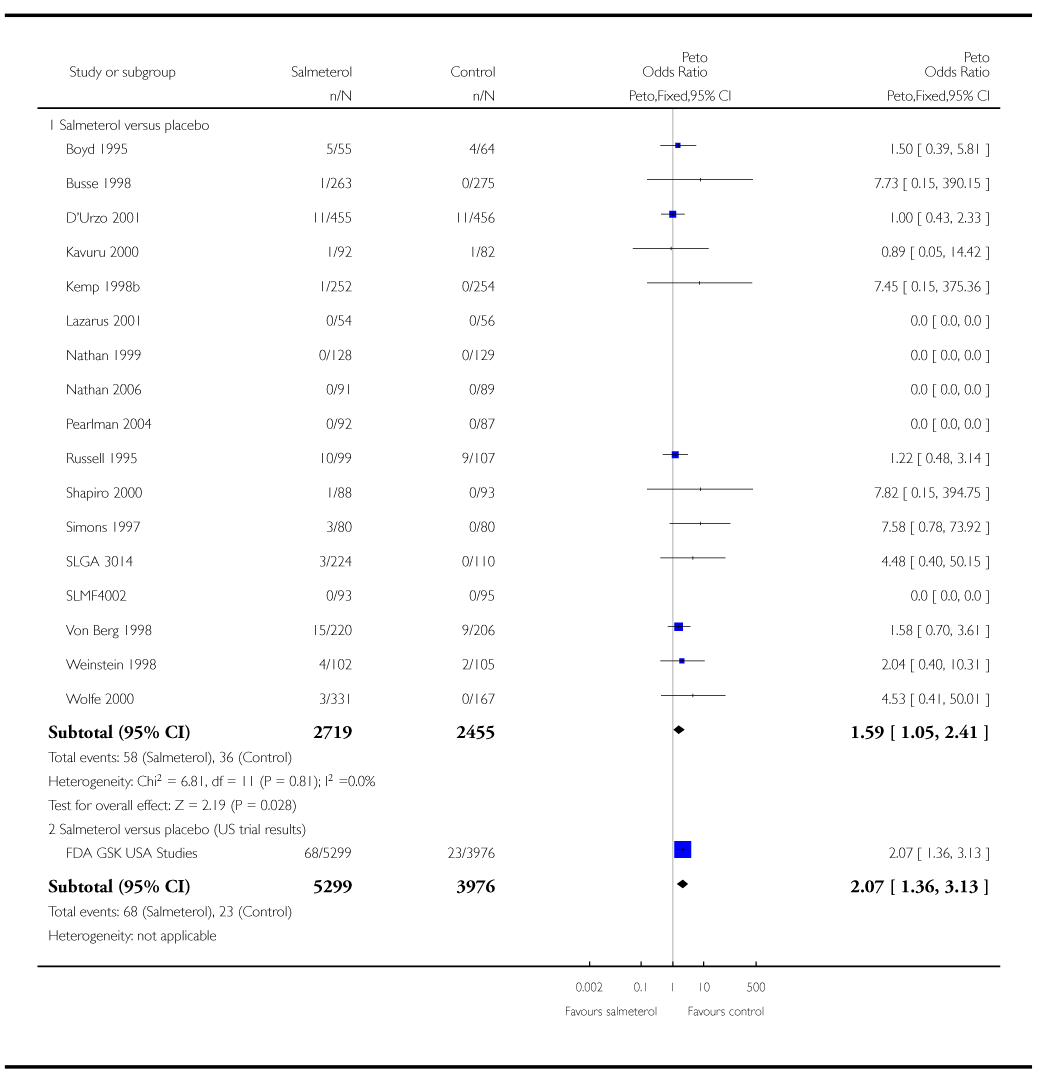

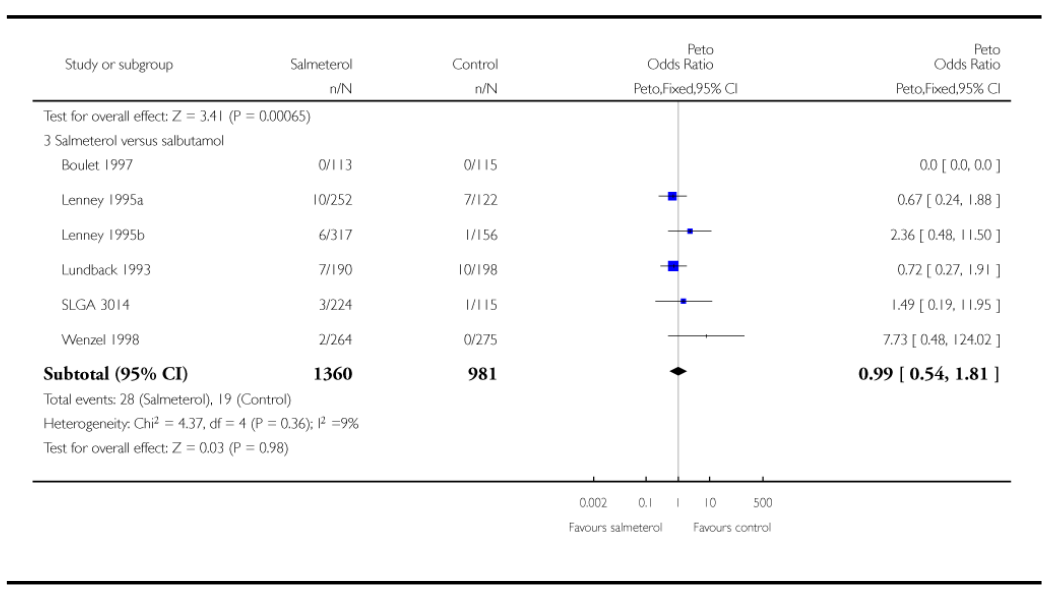

\section{Appendix 1: Pharmacology of beta2-agonists}

Beta $_{2}$-agonists are thought to cause bronchodilation primarily through binding beta $2^{-}$ adrenoceptors on airways smooth muscle (ASM), with subsequent activation of both membrane-bound potassium channels and a signalling cascade involving enzyme activation and changes in intracellular calcium levels following a rise in cyclic adenosine 
monophosphate (cAMP) (Barnes 1993). However, beta 2 -adrenoceptors are also expressed on a wide range of cell types where beta2-agonists may have a clinically significant effect including airway epithelium (Morrison 1993), mast cells, post capillary venules, sensory and cholinergic nerves and dendritic cells (Anderson 2006). Beta 2 -agonists will also cross-react to some extent with other beta-adrenoceptors including beta ${ }_{1}$-adrenoceptors on the heart. The in vivo effect of any beta 2 -agonist will depend on a number of factors relating to both the drug and the patient. The degree to which a drug binds to one receptor over another is known as selectivity, which can be defined as absolute binding ratios to different receptors in vitro, whilst functional selectivity is measured from downstream effects of drugs in different tissue types in vitro or in vivo. All of the beta 2 -agonists described thus far are more beta 2 selective than their predecessor isoprenaline in vitro. However, because attempts to differentiate selectivity between the newer agents are confounded by so many factors, it is difficult to draw conclusions about in vitro selectivity studies and probably best to concentrate on specific adverse side-effects in human subjects at doses which cause the same degree of bronchoconstriction. The potency of a drug refers to the concentration that achieves half the maximal receptor activation of which that drug is capable but it is not very important clinically as for each drug, manufacturers will alter the dose to try to achieve a therapeutic ratio of desired to undesired effects. In contrast efficacy refers to the ability of a drug to activate its receptor independent of drug concentration. Drugs that fully activate a receptor are known as full agonists and those that partially activate a receptor are known as partial agonists. Efficacy also is very much dependent on the system in which it is being tested and is affected by factors including the number of receptors available and the presence of other agonists and antagonists. Therefore, whilst salmeterol acts as a partial agonist in vitro, it causes a similar degree of bronchodilation to the strong agonist formoterol in stable asthmatic patients (Van Noord 1996), presumably because there are an abundance of well-coupled beta 2 -adrenoceptors available with few downstream antagonising signals. In contrast, with repetitive dosing formoterol is significantly better than salmeterol at preventing methacholine-induced bronchoconstriction (Palmqvist 1999). These differences have led to attempts to define the 'intrinsic efficacy' of a drug independent of tissue conditions (Hanania 2002), as shown in Table 2. The clinical significance of intrinsic efficacy remains unclear.

\section{Appendix 2: Possible mechanisms of increased asthma mortality with beta2-agonists}

\section{Direct toxicity}

This hypothesis states that direct adverse effects of beta 2 -agonists are responsible for an associated increase in mortality and most research in the area has concentrated on effects detrimental to the heart. Whilst it is often assumed that cardiac side-effects of beta 2 -agonists are due to cross-reactivity with beta ${ }_{1}$-adrenoceptors (i.e. poor selectivity), it is worth noting that human myocardium also contains an abundance of beta 2 -adrenoceptors capable of triggering positive chronotropic and inotropic responses (Lipworth 1992). Indeed, there is good evidence that cardiovascular side effects of isoprenaline and other beta 2 -agonists including salbutamol are mediated predominantly via cardiac beta 2 -adrenoceptors thus 
making the concept of in vitro selectivity less relevant (Arnold 1985; Hall 1989).

Generalised beta 2 -adrenoceptor activation can also cause hypokalaemia and it has been proposed that, through these and other actions beta 2 -agonists may predispose to lifethreatening dysrhythmias or cause other adverse cardiac effects (Brown 1983).

During the 1960s epidemic, most deaths occurred in patients with severe asthma and it was originally assumed that asthma and its sequelae, including hypoxia, were the primary cause of death. However, mucus plugging and hypoxia does not preclude a cardiac event as the final cause of death, and one might expect those with severe asthma to take more doses of a prescribed inhaler. As noted by Speizer and Doll, most deaths in the 1960s were in the 10-19 age group and "at these ages children have begun to act independently and may be particularly prone to misuse a self-administered form of treatment" (Speizer 1968). If toxicity were related to increasing doses of beta 2 -agonists one might expect most deaths to occur in hospital where high doses are typically used and this was not the case. One possible explanation for this anomaly was provided by animal experiments in which large doses of isoprenaline caused little ill effect in anaesthetised dogs with normal arterial oxygenation whereas much smaller doses caused fatal cardiac depression and asystole (although no obvious dysrhythmia) when hypoxic (Collins 1969; McDevitt 1974). It has been hypothesised therefore that such events would be less likely in hospital where supplemental oxygen is routinely given. The clinical relevance of these studies remains unclear although there is some evidence of a synergistic effect between hypoxia and salbutamol use in asthmatic patients in reducing total peripheral vascular resistance (Burggraaf 2001) - another beta ${ }_{2}$ mediated effect which could be detrimental to the heart during an acute asthma attack through a reduction in diastolic blood pressure. Other potential mechanisms of isoprenaline toxicity include a potential increase in mucous plugging and worsening of ventilation perfusion mismatch despite bronchodilation (Pearce 1990).

Further concerns about a possible toxic effect of beta 2 -agonists were raised during the New Zealand epidemic in the 1970s. In 1981 Wilson et al who first reported the epidemic reviewed 22 fatal cases of asthma and noted "In 16 patients death was seen to be sudden and unexpected. Although all were experiencing respiratory distress, most were not cyanosed and the precipitate nature of their death suggested a cardiac event, such as an arrest, inappropriate to the severity of their respiratory problem" (Wilson 1981). In humans, fenoterol causes significantly greater chronotropic, inotropic and electrocardiographic side effects than salbutamol in asthmatic patients (Wong 1990). Interestingly, across the same parameters fenoterol also causes more side-effects than isoprenaline (Burgess 1991).

In patients with mild asthma and without a bronchoconstrictor challenge, salmeterol and salbutamol cause a similar degree of near maximal bronchodilation at low doses (Bennett 1994). However, whilst as a one off dose salbutamol is typically used at 2 to 4 times the concentration of salmeterol, the dose equivalences for salmeterol versus salbutamol in increasing heart rate and decreasing potassium concentration and diastolic blood pressure were 17.7, 7.8 and 7.6 respectively (i.e. salmeterol had a greater effect across all parameters). Given the lower intrinsic efficacy of salmeterol (Table 2), these results highlight the importance of in vivo factors; one possible explanation for the difference is the 
increased lipophilicity of salmeterol compared to salbutamol contributing to higher systemic absorption (Bennett 1994).

When comparing increasing actuations of standard doses of formoterol and salmeterol inhalers in stable asthmatic patients, relatively similar cardiovascular effects are seen at lower doses (Guhan 2000). However, at the highest doses (above those recommended by the manufacturers) there were trends towards an increase in systolic blood pressure with formoterol; in comparison there was a trend towards a decrease in diastolic blood pressure and an increase in QTc interval with salmeterol although no statistical analysis of the difference was performed. In contrast in asthmatic patients with methacholine-induced bronchoconstriction there was no significant difference between salmeterol and formoterol in causing increased heart rate and QTc interval although formoterol caused significantly greater bronchodilation and hypokalaemia (Palmqvist 1999). Whilst there is good evidence of cardiovascular and metabolic side-effects with increasing doses of beta ${ }_{2}$-agonists, it is a little difficult to envisage serious adverse effects of this nature when using LABAs at manufacturer-recommended preventative doses. However, it is possible that some patients choose to use repeated doses of LABAs during exacerbations.

\section{Tolerance}

In this setting, the term tolerance refers to an impaired response to beta ${ }_{2}$-agonists in patients who have been using regular beta 2 -agonist treatment previously (Haney 2006). Tolerance is likely to result from a combination of reduced receptor numbers secondary to receptor internalisation and reduced production and also uncoupling of receptors to downstream signalling pathways following repeated activation (Barnes 1995). This phenomenon is likely to explain the beneficial reduction in systemic side effects seen with regular use of beta $2_{2}$ agonists including salbutamol after 1 to 2 weeks (Lipworth 1989). However, the same effect on beta ${ }_{2}$-adrenoceptors in the lung might be expected to produce a diminished response to the bronchodilating activity of beta 2 -agonists following regular use. In patients with stable asthma, whilst there is some evidence of tolerance to both salbutamol (Nelson 1977) and terbutaline (Weber 1982) other studies have been less conclusive (Harvey 1982; Lipworth 1989). However, evidence of tolerance to short and long-acting beta 2 -agonists in both protecting against and reducing bronchoconstriction is much stronger in the setting of an acute bronchoconstrictor challenge with chemical, allergen and 'natural' stimuli (Haney 2006; Lipworth 1997).

Studies comparing salmeterol and formoterol have shown that both cause tolerance compared to placebo but there was no significant difference between the drugs (Van der Woude 2001). There also appears to be little difference in the tolerance induced by regular formoterol and regular salbutamol treatment (Hancox 1999; Jones 2001). To the authors' knowledge no studies have looked specifically at the degree of tolerance caused by isoprenaline and fenoterol in the setting of acute bronchoconstriction. Tolerance to bronchodilation has been shown to clearly occur with addition of inhaled corticosteroids to salmeterol and formoterol (Lee 2003) and terbutaline (Yates 1996). There is conflicting evidence as to whether high dose steroids can reverse tolerance in the acute setting (Jones 2001; Lipworth 2000). 
At first glance the toxicity and tolerance hypotheses might appear incompatible as systemic and cardiovascular tolerance ought to protect against toxicity in the acute setting and there is good evidence that such tolerance occurs in stable asthmatic patients (Lipworth 1989).

However, whilst this study showed that changes in heart rate and potassium levels were blunted by previous beta 2 -agonist use, they were not abolished; furthermore, at the doses studied these side effects appear to follow an exponential pattern (Lipworth 1989). In contrast, in the presence of bronchoconstrictor stimuli the bronchodilator response to beta 2 agonists follows a flatter curve (Hancox 1999; Wong 1990) and as previously discussed this curve is shifted downwards by previous beta-agonist exposure (Hancox 1999). Thus, it is theoretically possible that in the setting of an acute asthmatic attack and strong bronchoconstricting stimuli, bronchodilator tolerance could lead to repetitive beta 2 -agonist use and ultimately more systemic side effects than would otherwise have occurred. Of course, other sequelae of inadequate bronchodilation including airway obstruction will be detrimental in this setting.

Whilst the tolerance hypothesis is often cited as contributing towards the asthma mortality epidemics it is difficult to argue that reduced efficacy of a drug can cause increased mortality relative to a time when that drug was not used at all. However, tolerance to the bronchodilating effect of endogenous circulating adrenaline is theoretically possible and there is also evidence of rebound bronchoconstriction when stopping fenoterol (Sears 1990), which may be detrimental. Furthermore, it appears that regular salbutamol treatment can actually increase airway responsiveness to allergen (Cockcroft 1993), a potentially important effect that could form a variant of the toxicity hypothesis. Differences between beta 2 agonists in this regard are unclear, but the combination of rebound hyperresponsiveness and tolerance of the bronchodilator effect with regular beta 2 -agonist exposure has been recently advocated as a possible mechanism to explain the association between beta 2 -agonists and asthma mortality (Hancox 2006a).

\section{Other explanations}

\section{Confounding by severity}

Historically, this hypothesis has been used extensively to try to explain the association between mortality and the use of fenoterol during the 1970s New Zealand epidemic and is still quoted today (Pearce 2007). The hypothesis essentially relies on the supposition that patients with more severe asthma are more likely to take either higher doses of betaagonists or a particular beta 2 -agonist (such as fenoterol) thereby explaining the association. This hypothesis was carefully ruled out in the three case-control studies by comparing the association between fenoterol and mortality in patients with varying severity of disease (Crane 1989; Grainger 1991; Pearce 1990). Furthermore, the hypothesis cannot explain the overall increase in mortality in the 1960s and 1970s, nor can it explain any significant increase in mortality (whether taking inhaled steroids or not) from randomised controlled trial data. 


\section{The delay hypothesis}

This hypothesis accepts that beta 2 -agonists or a particular beta 2 -agonist cause an increased risk of mortality but indirectly by causing patients to delay before getting medical help and further treatments including high dose steroids and oxygen. There is evidence that both salmeterol and formoterol can reduce awareness of worsening underlying inflammation (Bijl-Hofland 2001; McIvor 1998). It is difficult to rule out the delay hypothesis in either explaining or contributing towards both the asthma mortality epidemics and an association with regular use of LABAs. There is evidence that beta 2 -agonists with higher intrinsic efficacy are more effective at relieving bronchoconstriction in the acute setting and could paradoxically cause patients to delay seeking medical help for longer (Hanania 2007). For the delay hypothesis to explain the increase in mortality during the 1960s and 1970s, one has to imply that hospital treatment of asthma when mortality rates were low during the earlier years of the $20^{\text {th }}$ century was effective. It is difficult to say exactly how effective such treatment is likely to have been.

\section{Reduced corticosteroid treatment}

A slight but significant variation of the delay hypothesis suggests that patients who have separate beta2-agonists and corticosteroid inhalers may choose to take less corticosteroid because of better symptom control from the inhaled beta 2 -agonists and it is reduced corticosteroid treatment that contributes to a rise in mortality. It is rather difficult to see how this hypothesis explains the epidemics of asthma deaths in the 1960s and 1970s relative to the 1920s and 30s (Figure 1), given that corticosteroids were not used for the treatment of asthma in the earlier decades. If this hypothesis were to explain increased mortality from more recent randomised controlled trial data, one would not expect to see an increase in mortality in those taking LABAs alone.

\section{Appendix 3: Sources and search methods for the Cochrane Airways Group Specialised Register (CAGR)}

\section{Electronic searches: core databases}

\begin{tabular}{ll}
\hline Database & Frequency of search \\
MEDLINE (Ovid) & Weekly \\
EMBASE (Ovid) & Weekly \\
CENTRAL (the Cochrane Library) & Quarterly \\
PSYCHINFO (Ovid) & Monthly \\
CINAHL (EBSCO) & Monthly \\
AMED (EBSCO) & Monthly \\
\hline
\end{tabular}




\section{Handsearches: core respiratory conference abstracts}

\begin{tabular}{ll}
\hline Conference & Years searched \\
American Academy of Allergy, Asthma and Immunology (AAAAI) & 2001 onwards \\
American Thoracic Society (ATS) & 2001 onwards \\
Asia Pacific Society of Respirology (APSR) & 2004 onwards \\
British Thoracic Society Winter Meeting (BTS) & 2000 onwards \\
Chest Meeting & 2003 onwards \\
European Respiratory Society (ERS) & $1992,1994,2000$ onwards \\
International Primary Care Respiratory Group Congress (IPCRG) & 2002 onwards \\
Thoracic Society of Australia and New Zealand (TSANZ) & 1999 onwards \\
\hline
\end{tabular}

\section{MEDLINE search strategy used to identify trials for the CAGR}

\section{Asthma search}

1. exp Asthma/

2. asthma\$.mp.

3. (antiasthma $\$$ or anti-asthma\$).mp.

4. Respiratory Sounds/

5. wheez\$.mp.

6. Bronchial Spasm/

7. bronchospas\$.mp.

8. (bronch\$ adj3 spasm\$).mp.

9. bronchoconstrict\$.mp.

10. exp Bronchoconstriction/

11. (bronch\$ adj3 constrict\$).mp.

12. Bronchial Hyperreactivity/

13. Respiratory Hypersensitivity/

14. ((bronchial\$ or respiratory or airway $\$$ or lung $\$$ ) adj3 (hypersensitiv\$ or hyperreactiv\$ or allerg\$ or insufficiency)).mp.

15. ((dust or mite\$) adj3 (allerg\$ or hypersensitiv\$)).mp. 
16. or/1-15

\section{Filter to identify RCTs}

1. exp "clinical trial [publication type]"/

2. (randomised or randomised).ab,ti.

3. placebo.ab,ti.

4. dt.fs.

5. randomly.ab,ti.

6. trial.ab,ti.

7. groups.ab,ti.

8. or/1-7

9. Animals/

10. Humans/

11. 9 not (9 and 10)

12. 8 not 11

The MEDLINE strategy and RCT filter are adapted to identify trials in other electronic databases

\section{Appendix 4: Definition of serious adverse events (SAEs)}

A serious adverse event (SAE) is any adverse event occurring at any dose that results in any of the following outcomes:

1. Death

2. A life threatening adverse event

3. Inpatient hospitalisation or prolongation of existing hospitalisation

4. A disability/incapacity

5. A congenital anomaly in the offspring of a subject who received medication

6. Important medical events that may not result in death, be life-threatening, or require hospitalisation may be considered a serious adverse event when, based upon appropriate medical judgment, they may jeopardise the patient or subject and may require medical or surgical intervention to prevent one of the outcomes listed 
in this definition. Examples of such medical events include allergic bronchospasm requiring intensive treatment in an emergency room or at home, blood dyscrasias or convulsions that do not result in inpatient hospitalisation, or the development of medication dependency or medication abuse.

\section{Clarifications}

'Occurring at any dose' does not imply that the subject is receiving study medication.

Life-threatening means that the subject was, in the view of the investigator, at immediate risk of death from the event as it occurred.

This definition does not include an event that, had it occurred in a more severe form, might have caused death.

Hospitalisation for elective treatment of a pre-existing condition that did not worsen during the study is not considered an AE.

Complications that occur during hospitalisation are AEs. If a complication prolongs hospitalisation, the event is a SAE.

'Inpatient' hospitalisation means the subject has been formally admitted to a hospital for medical reasons. This may or may not be overnight. It does not include presentation at a casualty or emergency room.

With regard to criterion number 6 above, medical and scientific judgment should be used in deciding whether prompt reporting is appropriate in this situation.

\section{Events or outcomes not qualifying as SAEs}

The events or outcomes identified as asthma exacerbations will be recorded in the asthma exacerbations page of the case report form (CRF) page if they occur. However, these individual events or outcomes, as well as any sign, symptom, diagnosis, illness, and/or clinical laboratory abnormality that can be linked to any of these events or outcomes, are not reported to GW as SAEs even though such event or outcome may meet the definition of SAE, unless the following conditions apply: the investigator determines that the event or outcome qualifies as a SAE under criterion number 6 of the SAE definition (see Section 7.2, Definition of a SAE), or the event or outcome is in the investigator's opinion of greater intensity, frequency or duration than expected for the individual subject, or death occurring for any reason during a study, including death due to a disease-related event.

\section{WHAT'S NEW}

Last assessed as up-to-date: 17 August 2011. 


\begin{tabular}{ccc}
\hline Date & Event & Description \\
11 April 2013 & Amended & NIHR acknowledgement inserted \\
\hline
\end{tabular}

\section{HISTORY}

Protocol first published: Issue 1, 2007

Review first published: Issue 3, 2008

\begin{tabular}{|c|c|c|}
\hline Date & Event & Description \\
\hline 17 August 2011 & $\begin{array}{l}\text { New search } \\
\text { has been } \\
\text { performed }\end{array}$ & $\begin{array}{l}\text { New search in August } 2011 \text { but no new included studies. Minor edits made and } \\
\text { plain language summary rewritten }\end{array}$ \\
\hline 24 August 2009 & $\begin{array}{l}\text { New search } \\
\text { has been } \\
\text { performed }\end{array}$ & $\begin{array}{l}\text { New search in August } 2009 \text { but no new studies found. New Summary of } \\
\text { Findings tables added to show separate results for adults and children. Russell } \\
1995 \text { was previously wrongly classified as an adult study and this has now been } \\
\text { corrected. The total number of participants in the review has been altered to } \\
\text { reflect those who were randomised to one of the treatment arms included in this } \\
\text { review. There is no change in the conclusions of the review }\end{array}$ \\
\hline 20 December 2008 & Amended & $\begin{array}{l}\text { Primary analysis changed to Peto Odds Ratio. } \\
\text { Typographic errors in the number of trials and participants contributing to the } \\
\text { mortality and non-fatal serious adverse events have been corrected to match the } \\
\text { results in Figures } 5 \text { and } 7 \text {. The results are unchanged } \\
\text { NIHR Programme Grant Support has been acknowledged as external funding }\end{array}$ \\
\hline 2 July 2008 & $\begin{array}{l}\text { New search } \\
\text { has been } \\
\text { performed }\end{array}$ & $\begin{array}{l}\text { A new search was carried out on July } 1 \text { st } 2008 \text { but no new studies have been } \\
\text { included } \\
\text { Additional figures have been added comparing the results of published adverse } \\
\text { events and the complete data set available to us at present. This is included in an } \\
\text { updated discussion section. The risk of bias tables have also been amended to } \\
\text { reflect all the data available for serious adverse events in each study (previously } \\
\text { the risk of selective reporting was classified according to the paper } \\
\text { publications). The ongoing GSK study has now been excluded as it is a single } \\
\text { dose study. An error in the data entry for SNS in Figure } 7 \text { has been corrected } \\
\text { No changes have been made to the conclusions of the review. }\end{array}$ \\
\hline
\end{tabular}

\section{References to studies included in this review}

Adinoff 1998 [published data only]. Adinoff A, Schwartz H, Rickard K, Yancey S, Swearingen B. Salmeterol compared with current therapies in chronic asthma. Journal of Family Practice. 1998; 47(4):278-84. [PubMed: 9789513]

Boulet 1997 [published data only]. Boulet LP, Laviolette M, Boucher S, Knight A, Hébert J, Chapman KR. A twelve week comparison of salmeterol and salbutamol in the treatment of mildto-moderate asthma: a Canadian multicentre study. Journal of Allergy and Clinical Immunology. 1997; 99(1 Pt 1):13-21. [PubMed: 9003206]

Boyd 1995 [published data only]. Boyd G. Salmeterol xinafoate in asthmatic patients under consideration for maintenance oral corticosteroid therapy. European Respiratory Journal. 1995; 8:1494-98. [PubMed: 8575574]

Britton 1992 [published data only]. Britton MG, Earnshaw JS, Palmer JBD. A twelve month comparison of salmeterol with salbutamol in asthmatic patients. European Respiratory Journal. 1992; 5(9):1062-7. [PubMed: 1426215] * SLGT02. Inhaled GR33343G In Reversible Airways Obstruction - Efficacy Over 3 Months and Safety Over 12 Months. http://ctr.gsk.co.uk/Summary/ salmeterol/III.SLGT02.pdf 
Busse 1998 [published data only]. Busse WW, Casale TB, Murray JJ, Petrocella V, Cox F, Rickard K. Efficacy, safety, and impact on quality of life of salmeterol in patients with moderate persistent asthma. American Journal of Managed Care. 1998; 4(11):1579-87. [PubMed: 10338904]

Chervinsky 1999 [published data only]. Chervinsky P, Goldberg P, Galant S, Wang Y, Arledge T, Welch MB, et al. Long-term cardiovascular safety of salmeterol powder pharmacotherapy in adolescent and adult patients with chronic persistent asthma: a randomized clinical trial. Chest. 1999; 115(3):642-8. [PubMed: 10084469]

D’Alonzo 1994 [published data only]. D'Alonzo GE. Efficacy of inhaled salmeterol in the treatment of asthma. European Respiratory Review. 1995; 5:128-32.* D'Alonzo GE, Nathan RA, Henchowicz MD. Salmeterol xinafoate as maintenance therapy compared with albuterol in patients with asthma. JAMA. 1994; 271(18):1412-6. [PubMed: 7909853]

D’Urzo 2001 [published data only]. D’Urzo AD, Chapman KR, Cartier A, Hargreave FE, Fitzgerald M, Tesarowski D. Effectiveness and safety of salmeterol in non-specialist practice settings. Chest. 2001; 119(3):714-9. [PubMed: 11243947] * SLGQ94 (521/180). A multicenter, randomized, double-blind, parallel-group trial to evaluate the long-term efficacy and safety of inhaled salmeterol 50ìg BID compared to short-acting â2-agonists as-needed in adult patients with asthma. http://ctr.gsk.co.uk/Summary/salmeterol/studylist.asp

FDA GSK USA Studies [published data only]. GlaxoSmithKline. Safety of Long-Acting BetaAgonist Bronchodilators. http://www.fda.gov/ohrms/dockets/ac/05/briefing/2005-4148B1.01.01GSK-Serevent.pdf 2005

Kavuru 2000 [published and unpublished data]. Edwards T, Gross G, Mitchell D, Chervinsky P, Woodring A, Baitinger L, et al. The salmeterol xinafoate/fluticasone propionate dry powder combination product via diskus(r) inhaler improves asthma control compared to salmeterol xinafoate or fluticasone propionate dry powder alone. American Journal of Respiratory and Critical Care Medicine. 1998; 157(3 Suppl):A414.* Kavuru M, Melamed J, Gross G, Laforce C, House K, Prillaman B, et al. Salmeterol and fluticasone propionate combined in a new powder inhalation device for the treatment of asthma: a randomized, double-blind, placebo-controlled trial. Journal of Allergy and Clinical Immunology. 2000; 106(6 (pt 1)):1108-16. [PubMed: 11112894] Nathan R, Woodring A, Baitinger L, Prillaman B, Faris M, House K, et al. The salmeterol/fluticasone propionate Diskus combination decreases the incidence of exacerbations compared to treatment with salmeterol or fluticasone propionate alone. European Respiratory Journal. 1999; 14:123S.SFCA3002. A randomized, double-blind, parallel-group trial evaluating safety and efficacy of salmeterol 50mcg BID and fluticasone propionate $100 \mathrm{mcg}$ BID individually and in combination and placebo in subjects with asthma. http://ctr.gsk.co.uk 2005

Kemp 1998a [published data only]. * Kemp J, Wolfe J, Grady J, LaForce C, Stahl E, Arlidge T, et al. Salmeterol powder compared with albuterol aerosol as maintenance therapy for asthma in adolescent and adult patients. Clinical Therapeutics. 1998; 20(2):270-82. [PubMed: 9589818] Wolfe J, LaForce C, Chervinsky P, Galant S, Lumry W, Noonan M, et al. Inhaled salmeterol powder compared with albuterol aerosol given regularly or as needed for asthma. Annals of Allergy, Asthma \& Immunology. 1995; 74:52.

Kemp 1998b [published data only]. Kemp JP, Cook DA, Incaudo GA, Corren J, Kalberg C, Emmett A, et al. Salmeterol improves quality of life in patients with asthma requiring inhaled corticosteroids. Journal of Allergy and Clinical Immunology. 1998; 101:188-95. [PubMed: 9500751]

Lazarus 2001 [published data only]. Lazarus SC, Boushey HA, Fahy JV, Chinchilli VM, Lemanske RF Jr, Sorkness CA, et al. Long-acting \{beta\}2-agonist monotherapy vs continued therapy with inhaled corticosteroids in patients with persistent asthma: a randomized controlled trial. JAMA. 2001; 285(20):2583-93. [PubMed: 11368732]

Lenney 1995a [published data only]. Lenney W, Pedersen S, Boner AL, Ebbutt A, Jenkins MM. Efficacy and safety of salmeterol in childhood asthma. European Journal of Pediatrics. 1995; 154:983-90. [PubMed: 8801107] * SLPT01. A Multicentre, Randomized, Double-Blind, 3Limbed, Parallel-Group Study Comparing the Efficacy and Tolerability of Salmeterol Xinafoate 25ìg and 50ìg BID With Salbutamol 200ìg BID Given via Pressurized Inhalers in Children With Reversible Airways Obstruction. (3-Month Efficacy and Safety Study.). http://ctr.gsk.co.uk/ Summary/salmeterol/III.SLPT01.pdfSMS40093 (SLPT01). A multi-centre, randomised, double 
blind, three limbed, parallel-group study comparing the efficacy and tolerability of salmeterol xinafoate 25ìg and 50ìg bd with salbutamol 200ìg bd given via pressurised inhalers in children with reversible airways obstruction. (Results of the final nine months safety phase.). http:// ctr.gsk.co.uk/Summary/salmeterol/studylist.asp

Lenney 1995b [published data only]. Lenney W, Pedersen S, Boner AL, Ebbutt A, Jenkins MM. Efficacy and safety of salmeterol in childhood asthma. European Journal of Pediatrics. 1995; 154:983-90. [PubMed: 8801107] * SLPT02. A Multi-centre, Randomised, Double-Blind, 3Limbed, Parallel-Group Study Comparing the Efficacy and Tolerability of Dry Powder Formulations of Salmeterol Xinafoate 25ìg and 50ìg Twice Daily With Salbutamol 200ìg Twice Daily Given in Children With Reversible Airways Obstruction. http://ctr.gsk.co.uk/Summary/ salmeterol/III.SLPT02.pdf

Lundback 1993 [published data only]. Lundback B, Rawlinson DW, Palmer JB. Twelve month comparison of salmeterol and salbutamol as dry powder formulations in asthmatic patients. European Study Group. Thorax. 1993; 48(2):148-53. [PubMed: 8493629] * SLGT06. Inhaled GR33343G in Reversible Airways Obstruction - Efficacy and Safety Over Three Months: A Double-Blind, Parallel-Group Study Comparing Dry Powder Formulations of Inhaled GR33343G (50mcg) Administered Twice a Day and Inhaled Salbutamol (400mcg) Administered Four Times a Day. http://ctr.gsk.co.uk/Summary/salmeterol/studylist.asp

Nathan 1999 [published data only]. Nathan R, Pinnas J, Schwartz H, Grossman J, Yancey S, Emmett A, et al. A six-month, placebo-controlled comparison of the safety and efficacy of salmeterol or beclomethasone for persistent asthma. Annals of Allergy. 1999; 82(6):521-9.

Nathan 2006 [published data only]. Edin HM, Payne E, Herrle MR, Schoaf L, Mather DB, Scott CA, et al. Salmeterol/fluticasone propionate combination via HFA MDI improves quality of life. Journal of Allergy and Clinical Immunology. 2001; 107(2):S246.Nathan RA, Mitchell D, Condemi J, Heller A, Schoaf L, Herrle M, et al. Cardiovascular and hypothalmic-pituitaryadrenal axis safety of fluticasone propionate/salmeterol HFA MDI in adolescent and adult patients with asthma. American Journal for Respiratory and Critical Care Medicine. 2001; 163(5):A863.* Nathan RA, Rooklin A, Schoaf L, Scott C, Ellsworth A, House K, et al. Efficacy and tolerability of fluticasone propionate/salmeterol administered twice daily via hydrofluoroalkane 134a metered-dose inhaler in adolescent and adult patients with persistent asthma: a randomized, double-blind, placebo-controlled, 12-week study. Clinical Therapeutics. 2006; 28(1):73-85. [PubMed: 16490581] Pearlman DS, Kent E, Lanz MJ, Peden D, Baitinger L, Herrle M, et al. Fluticasone propionate/salmeterol HFA MDI has a rapid onset of effect in asthmatics treated with short- or long-acting beta-agonists (BA) or inhaled corticosteroids (ICS). Amercian Journal of Respiratory and Critical Care Medicine. 2001; 163(5):A865.Rooklin A, Elkayam D, Weiler J, Windom H, Schoaf L, Scott C, et al. The fluticasone propionate/salmeterol HFA MDI is significantly more efficacious in treating asthma than placebo HFA MDI, fluticasone propionate CFC MDI or salmeterol CFC MDI. Journal of Allergy and Clinicial Immunology. 2001; 107(2):100s.SAS30004. A randomized, double-blind, placebo-controlled, parallel-group 12-week trial evaluating the safety and efficacy of the salmeterol/fluticasone propionate combination in GR106642X MDI, 50/250 mcg BID, and salmeterol in propellant 11/12 MDI, 50mcg BID, fluticasone propionate in propellant 11/12 MDI, $250 \mathrm{mcg}$ BID, and placebo propellant GR106642X MDI in adult and adolescent subjects with asthma. 2005. http:// ctr.gsk.co.uk

Pearlman 1992 [published data only]. Arledge T, Liddle D, Stahl E, Rossing T. Salmeterol does not cause tolerance during long term asthma therapy. Journal of Allergy and Clinical Immunology. 1996; 98(6 Pt 1):1116-9. [PubMed: 8977514] Nathan R, Seltzer J, Kemp J, Chervinsky P, Alexander W, Liddle R, et al. Safety of salmeterol in the maintenance treatment of asthma. Annals of Allergy, Asthma \& Immunology. 1995; 75(3):243-8.Pearlman DS. Long-acting beta2agonist salmeterol compared with albuterol in maintenance asthma therapy. Annals of Allergy, Asthma \& Immunology. 1995; 75(2):180-4.* Pearlman DS, Chervinsky P, LaForce C, Seltzer JM, Southern DL, Kemp JP, et al. A comparison of salmeterol with albuterol in the treatment of mild-to-moderate asthma. New England Journal of Medicine. 1992; 327(20):1420-5. [PubMed: 1357554] Pearlman DS, Liddle R. Controlling asthma symptoms: salmeterol compared with salbutamol in large-scale multicentre studies. European Respiratory Review. 1994; 4(21):301-5. 
Pearlman 2004 [published data only]. * Pearlman DS, Peden D, Condemi JJ, Weinstein S, White M, Baitinger L, et al. Efficacy and safety of fluticasone propionate/salmeterol HFA 134A MDI in patients with mild-to-moderate persistent asthma. Journal of Asthma. 2004; 41(8):797-806. [PubMed: 15641629] SAS30003. A stratified, randomised, double-blind, placebo-controlled, parallel-group, 12-week trial evaluating the safety and efficacy of the salmeterol/fluticasone propionate combination in HFA 134a MDI, 42/88 mcg BID, and salmeterol in propellant 11/12 MDI, $42 \mathrm{mcg}$ BID, fluticasone propionate in propellant 11/12 MDI, $88 \mathrm{mcg}$ BID, and placebo propellant HFA 134a MDI in adult and adolescent subjects with asthma. 2005. http:// ctr.gsk.co.ukWeinstein SF, Pearlman DS, Condemi JJ, Herrle MR, Scott CA, Payne JE, et al. Superior efficacy of the fluticasone propionate/salmeterol 88/42 mcg HFA-MDI combination product versus the individual components in asthmatics previously treated with either short- or long-acting beta2-agonists or inhaled corticosteroids. Journal of Allergy \& Clinial Immunology. 2001; 107(2):S102.

Rosenthal 1999 [published data only]. Rosenthal R, Busse W, Kemp J, Baker J, Kalberg C, Emmett A, et al. Effect of long-term salmeterol therapy compared with as-needed albuterol use on airway hyperresponsiveness. Chest. 1999; 116(3):595-602. [PubMed: 10492259]

Russell 1995 [published data only]. Russell G, Williams DA, Weller P, Price JF. Salmeterol xinafoate in children on high dose inhaled steroids. Annals of Allergy, Asthma \& Immunology. 1995; 75(5):423-8.* SALMP/AH91/D89. A phase III, multi-centre, double-blind, placebo controlled, parallel group study assessing the efficacy and safety of inhaled salmeterol xinafoate (Serevent ${ }^{\mathrm{TM}}$ ) 50 micrograms BD via the Diskhaler ${ }^{\mathrm{TM}}$ when added to the existing treatment of moderate to severe asthmatic children. http://ctr.gsk.co.uk/Summary/salmeterol/studylist.asp

Shapiro 2000 [published data only]. * SFCA3003. A randomized, double-blind, parallel-group trial evaluating safety and efficacy of salmeterol $50 \mathrm{mcg}$ BID and fluticasone propionate $250 \mathrm{mcg}$ BID individually and in combination and placebo in subjects with asthma. 2005. http:// ctr.gsk.co.uk/Summary/fluticasone.salmeterol/III.SFCA3003.pdfShapiro G, Lumry W, Wolfe J, Given J, White M, Woodring A, et al. Combined salmeterol $50 \mathrm{mcg}$ and fluticasone propionate $250 \mathrm{mcg}$ in the Diskus device for the treatment of asthma. American Journal of Respiratory and Critical Care Medicine. 2000; 161:527-34. [PubMed: 10673196]

Simons 1997 [published data only]. Malozowski S, Stadel BV, Pian LP. Comparison of beclomethasone, salmeterol, and placebo in children with asthma. New England Journal of Medicine. 1998; 339(10):704-5. [PubMed: 9729145] Simons FE. A comparison of beclomethasone, salmeterol, and placebo in children with asthma. Canadian Beclomethasone Dipropionate-Salmeterol Xinafoate Study Group. New England Journal of Medicine. 1997; 337(23):1659-65. [PubMed: 9385125] Simons FER, Dolovich J, Moote DW, Mazza JA, Lyttle $\mathrm{B}$, Becker AB, et al. A one year placebo controlled study of the efficacy and safety of beclomethasone DP versus salmeterol in glucocorticoid naive children with asthma. Journal of Allergy and Clinical Immunology. 1997; 99:S402.* SMS40065 (521/120 [SLPT10]). Efficacy and safety of long-term inhaled salmeterol and beclomethasone dipropionate in corticosteroidnaïve children with mild to moderate, chronic, stable asthma. http://ctr.gsk.co.uk/Summary/ salmeterol/studylist.asp

SLGA 3014 [published data only]. GlaxoSmithKline. Serevent submission to the FDA. 2001. http:// www.fda.gov/cder/foi/nda/98/20692S1,2.Serevent.medr.P2.pdf

SLMF4002 [published data only]. * SLMF 4002. Efficacy and safety of salmeterol in patients with asthma controlled with inhaled corticosteroids. http://ctr.gsk.co.uk/Summary/salmeterol/ IV.SLMF4002.pdf

SMART 2006 [published data only]. GlaxoSmithKline. Safety of Long-Acting Beta-Agonist Bronchodilators. 2005. http://www.fda.gov/ohrms/dockets/ac/05/briefing/2005-4148B1.01.01GSK-Serevent.pdfKnobil K, Yancey S, Kral K, Rickard K. Salmeterol Multicentre Asthma Research Trial (SMART): results from an interim analysis. Chest. 2003; 124:335s.Nelson HS, Weiss ST, Bleecker ER, Yancey SW, Dorinsky PM, the SMART Study Group. The Salmeterol Multicenter Asthma Research Trial: a comparison of usual pharmacotherapy for asthma or usual pharmacotherapy plus salmeterol. Chest. 2006; 129(1):15-26. [PubMed: 16424409] Rickard, KA. safety alert - serevent (salmeterol xinafoate). 2003. www.fda.gov* SLGA5011 SMART. SMART: a double-blind, randomized, placebo-controlled surveillance study of asthma event outcomes in subjects receiving either usual pharmacotherapy of asthma or usual 
pharmacotherapy plus salmeterol 42mcg twice daily. 2006. http://www.ctr.gsk.co.ukWooltorton E. Salmeterol (Serevent) asthma trial halted early. Canadian Medical Association Journal. 2003; 168(6):738. [PubMed: 12642431]

SNS 1993 [published data only]. Castle W, Fuller R, Hall J. Serevent nationwide surveillance study:Comparison of salmeterol with salbutamol in asthmatic patients who require regular bronchodilator treatment. BMJ. 1993; 306:1034-7. [PubMed: 8098238] * SNS-D920619. Serevent Nationwide Surveillance (SNS) Trial. http://ctr.gsk.co.uk/Summary/salmeterol/ IV.SNS.D920619.pdf

Von Berg 1998 [published data only]. * SLGB3019 (SLPT09). A multicentre, randomised, doubleblind, parallel group study comparing the efficacy and safety of inhaled salmeterol xinafoate 50 mcg bd with that of salbutamol $200 \mathrm{mcg}$ to use "as required" from Diskhalers for twelve months in children with asthma. http://ctr.gsk.co.uk/Summary/salmeterol/III.SLGB3019.pdfVon Berg A, De Blic J, La Rosa M. Regular salbutamol xinafoate compared with salbutamol as required in children with moderate asthma. American Journal of Respiratory and Critical Care Medicine. 1996; 4:A76.Von Berg A, De Blic J, La Rosa M, Kaad PH, Moorat A. A comparison of regular salmeterol vs 'as required' salbutamol therapy in asthmatic children. Respiratory Medicine. 1998; 92(2):292-9. [PubMed: 9616529]

Weinstein 1998 [published data only]. GlaxoSmithKline. Safety of Long-Acting Beta-Agonist Bronchodilators. 2005. www.fda.gov/ohrms/dockets/ac/05/briefing/2005-4148B1.01.01-GSKSerevent.pdfPearlman DS, Bronsky E, Chervinsky P. Inhaled salmeterol powder compared with placebo administered over 12 weeks to children with mild to moderate asthma. American Journal of Respiratory and Critical Care Medicine. 1996; 153(4):A76.* Weinstein S, Pearlman D, Bronsky E, Byrne A, Arledge T, Liddle R, et al. Efficacy of salmeterol xinafoate powder in children with chronic persistent asthma. Annals of Allergy, Asthma \& Immunology. 1998; 81(1): $51-8$.

Wenzel 1998 [published data only]. Cox F, Goodwin B, Wenzel S, Rickard K, Kalberg C, Emmett A. Asthma specific quality of life in patients treated with salmeterol or albuterol. Journal of Allergy and Clinical Immunology. 1996; 97:256.Goodwin R, Cox F, Lumry W, Rickard K, Kalberg C, Emmett A. The impact of salmeterol versus albuterol on disease specific quality of life in mild to moderate asthmatics. Journal of Allergy and Clinical Immunology. 1996; 97(Pt 3): 256.* Wenzel SE, Lumry W, Manning M, Kalberg C, Cox F, Emmett A, et al. Efficacy, safety, and effects on quality of life of salmeterol versus albuterol in patients with mild to moderate persistent asthma. Annals of Allergy. 1998; 80(6):463-70.

Wolfe 2000 [published and unpublished data]. SLGA3010. A randomized, double-blind, doubledummy, comparative clinical trial of salmeterol $50 \mathrm{mcg}$ via the Diskus and salmeterol $50 \mathrm{mcg}$ via the metered-dose inhaler versus placebo for 12 weeks in adolescent and adult subjects with mildto-moderate asthma. http://ctr.gsk.co.uk/Summary/salmeterol/III.SLGA3010.pdfSLGA3011. A randomized, double-blind, double-dummy, comparative clinical trial of salmeterol $50 \mathrm{mcg}$ via the Diskus and salmeterol $50 \mathrm{mcg}$ via the metered-dose inhaler versus placebo for twelve weeks in adolescent and adult subjects with mild-to-moderate asthma. http://ctr.gsk.co.uk/Summary/ salmeterol/III.SLGA3011.pdf* Wolfe J, Kreitzer S, Chervinsky P, Lawrence M, Wang Y, Reilly $\mathrm{D}$, et al. Comparison of powder and aerosol formulations of salmeterol in the treatment of asthma. Annals of Allergy. 2000; 84(3):334-40.

\section{References to studies excluded from this review}

Bagnato 1996 [published data only]. Bagnato GF, Mileto A, Gulli S, Oriti S, Di Cesare E, Cinquegrani M, et al. Acute cardiovascular effects of salmeterol in subjects with stable bronchial asthma. Monaldi Archives for Chest Disease. 1996; 51(4):275-8. [PubMed: 8909010]

Beach 1992 [published data only]. Beach JR, Young CL, Stenton SC, Avery AJ, Walters EH, Hendrick DJ. A comparison of the speeds of action of salmeterol and salbutamol in reversing methacholine-induced bronchoconstriction. Pulmonary Pharmacology. 1992; 5(2):133-5. [PubMed: 1611231]

Beach 1993 [published data only]. Beach JR, Young CL, Harkawat R, Gardiner PV, Avery AJ, Coward GA, et al. Effect on airway responsiveness of six weeks treatment with salmeterol. Pulmonary Pharmacology. 1993; 6(2):155-7. [PubMed: 8102918] 
Blake 1999 [published data only]. Blake K, Pearlman DS, Scott C, Wang Y, Stahl E, Arledge T. Prevention of exercise-induced bronchospasm in pediatric asthma patients: a comparison of salmeterol powder with albuterol. Annals of Allergy, Asthma \& Immunology. 1999; 82(2):20511.

Bons 1992 [published data only]. Bons J, Pappo M. Salmeterol and prolonged treatment of asthma: international clinical data. Revue des Maladies Respiratoires. 1992; 9(Suppl 1):R15-8. [PubMed: 1350364]

Booth 1993 [published data only]. Booth H, Fishwick K, Harkawat R, Devereux G, Hendrick DJ, Walters EH. Changes in methacholine induced bronchoconstriction with the long acting beta 2 agonist salmeterol in mild to moderate asthmatic patients. Thorax. 1993; 48(11):1121-4. [PubMed: 8296255]

Boulet 1997b [published data only]. Boulet LP, Turcotte H, Boutet M, Dube J, Gagnon M, Laviolette M. Influence of salmeterol on chronic and allergen-induced airway inflammation in mild allergic asthma: a pilot study. Current Therapeutic Research, Clinical \& Experimental. 1997; 58(4):24059.

Bousquet 1996 [published data only]. Bousquet J, Aubert B, Bons J. Comparison of salmeterol with disodium cromoglycate in the treatment of adult asthma. Annals of Allergy, Asthma \& Immunology. 1996; 76(2)

Brambilla 1994 [published data only]. Brambilla C, Chastang C, Georges D, Bertin L. Salmeterol compared with slow-release terbutaline in nocturnal asthma. A multicenter, randomized, doubleblind, double-dummy, sequential clinical trial. French Multicenter Study Group. Allergy. 1994; 49(6):421-6. [PubMed: 7915501]

Bronsky 1994 [published data only]. Bronsky EA, Kemp JP, Orgel HA, Bierman CW, Tinkelman DG, Van As A, et al. A 1-week dose-ranging study of inhaled salmeterol in patients with asthma. Chest. 1994; 105(4):1032-7. [PubMed: 7909285]

Bronsky 1999 [published data only]. Bronsky EA, Pearlman DS, Pobiner BF, Scott C, Wang Y, Stahl E. Prevention of exercise-induced bronchospasm in pediatric asthma patients: a comparison of two salmeterol powder delivery devices. Pediatrics. 1999; 104(3 Pt 1):501-6. [PubMed: 10469776]

Busse 1999 [published data only]. Busse W, Nelson H, Wolfe J, Kalberg C, Yancey SW, Rickard KA. Comparison of inhaled salmeterol and oral zafirlukast in patients with asthma. Journal of Allergy and Clinical Immunology. 1999; 103(6):1075-80. [PubMed: 10359889]

Cartier 1993 [published data only]. Cartier A, Ghezzo H, L’Archeveque J, Trudeau C, Malo JL. Duration and magnitude of action of 50 and 100 micrograms of inhaled salmeterol in protecting against bronchoconstriction induced by hyperventilation of dry cold air in subjects with asthma. Journal of Allergy and Clinical Immunology. 1993; 92(3):488-92. [PubMed: 8360399]

Castle 1992 [published data only]. Castle WM. Review of salmeterol international safety data base. European Respiratory Journal. 1992; 5(Suppl 15):318S.

Cazzola 2002 [published data only]. Cazzola M, Califano C, Di Perna F, D’Amato M, Terzano C, Matera MG, et al. Acute effects of higher than customary doses of salmeterol and salbutamol in patients with acute exacerbation of COPD. Respiratory Medicine. 2002; 96(10):790-5. [PubMed: 12412978]

Ceugniet 1997 [published data only]. Ceugniet F, Cauchefer F, Fragneaud C, Evano-Celli I. Prophylactic treatment of exercise-induced asthma in children: salmeterol or sodium cromoglycate single dose before exercise? Annales De Pediatrie. 1997; 44(9):625-34.

Cheung 1992 [published data only]. Cheung D, Timmers MC, Zwinderman AH, Bel EH, Dijkman JH, Sterk PJ. Long-term effects of a long-acting beta 2-adrenoceptor agonist, salmeterol, on airway hyperresponsiveness in patients with mild asthma. New England Journal of Medicine. 1992; 327(17):1198-203. [PubMed: 1357550]

Chopra 2005 [published data only]. Chopra N, Williams M, Rimmer M, Kahl L, Jenkins M, Teams SMOaSIS. Salmeterol HFA is as effective as salmeterol CFC in children and adults with persistent asthma. Respiratory Medicine. 2005; 99(Suppl 1):S1-10. [PubMed: 15777603]

Cloosterman 2001 [published data only]. Cloosterman SG, Bijl-Hofland ID, Van Herwaarden CL, Akkermans RP, Van Den Elshout FJ, Folgering HT, et al. A placebo-controlled clinical trial of 
regular monotherapy with short-acting and long-acting beta(2)-agonists in allergic asthmatic patients. Chest. 2001; 119(5):1306-15. [PubMed: 11348933]

Crompton 1999 [published data only]. Crompton GK, Ayres JG, Basran G, Schiraldi G, Brusasco V, Eivindson A, et al. Comparison of oral bambuterol and inhaled salmeterol in patients with symptomatic asthma and using inhaled corticosteroids. American Journal of Respiratory and Critical Care Medicine. 1999; 159(3):824-8. [PubMed: 10051257]

D'Alonzo 1995 [published data only]. D'Alonzo GE. Efficacy of inhaled salmeterol in the treatment of asthma. European Respiratory Review. 1995; 5(27):128-32.

D’Urzo 1998 [published data only]. D’Urzo A, Chapman KR, Cartier A, Fitzgerald M, Hargreave FE, Tesarowski D. Safety and efficacy of salmeterol in a post-marketing surveillance trial in asthmatics. American Journal of Respiratory and Critical Care Medicine. 1998; 157(3 Suppl):A402.

Dahl 1991 [published data only]. Dahl R, Earnshaw JS, Palmer JB. Salmeterol: a four week study of a long-acting beta-adrenoceptor agonist for the treatment of reversible airways disease. European Respiratory Journal. 1991; 4(10):1178-84. [PubMed: 1687131]

Dekhuijzen 2006 [published data only]. Dekhuijzen PNR. Caution recommended in prescribing long-acting beta2-adrenergic agonists to patients with asthma. Nederlands Tijdschrift voor Geneeskunde. 2006; 150(16):889-91. [PubMed: 16686087]

Demirkan 2000 [published data only]. Demirkan K, Tolley E, Mastin T, Soberman J, Burbeck J, Self T. Salmeterol administration by metered-dose inhaler alone vs metered-dose inhaler plus valved holding chamber. Chest. 2000; 117(5):1314-8. [PubMed: 10807816]

De Oliveira 1998 [published data only]. De Oliveira MA, Jardim JR, Faresin SM, Lucas SR, Nery LE. Efficacy of and tolerance to salmeterol compared to salbutamol in patients with bronchial asthma. Revista Da Associacao Medica Brasileira. 1998; 44(3):169-75. [PubMed: 9755543]

Deykin 2007 [published data only]. Deykin A, Wechsler ME, Boushey HA, Chinchilli VM, Kunselman SJ, Craig TJ, et al. Combination therapy with a long-acting beta-agonist and a leukotriene antagonist in moderate asthma. American Journal of Respiratory \& Critical Care Medicine. 2007; 175(3):228-34. [PubMed: 16973987]

Edelman 2000 [published data only]. Edelman JM, Turpin JA, Bronsky EA, Grossman J, Kemp JP, Ghannam AF, et al. Oral montelukast compared with inhaled salmeterol to prevent exerciseinduced bronchoconstriction. A randomized, double-blind trial. Annals of Internal Medicine. 2000; 132(2):97-104. [PubMed: 10644288]

Eryonucu 2005 [published data only]. Eryonucu B, Uzun K, Guler N, Tuncer M, Sezgi C. Comparison of the short-term effects of salmeterol and formoterol on heart rate variability in adult asthmatic patients. Chest. 2005; 128(3):1136-9. [PubMed: 16162698]

Faurschou 1994 [published data only]. Faurschou P, Engel AM, Haanaes OC. Salmeterol in two different doses in the treatment of nocturnal bronchial asthma poorly controlled by other therapies. Allergy. 1994; 49(10):827-32. [PubMed: 7709991]

Faurschou 1996 [published data only]. Faurschou P, Steffensen I, Jacques L. Effect of addition of inhaled salmeterol to the treatment of moderate-to-severe asthmatics uncontrolled on high-dose inhaled steroids. European Respiratory Study Group. European Respiratory Journal. 1996; 9(9): 1885-90. [PubMed: 8880107]

Fjellbirkeland 1994 [published data only]. Fjellbirkeland L, Gulsvik A, Palmer JB. The efficacy and tolerability of inhaled salmeterol and individually dose-titrated, sustained-release theophylline in patients with reversible airways disease. Respiratory Medicine. 1994; 88(8):599-607. [PubMed: 7991885]

Fuller 1995 [published data only]. Fuller R. Safety of salmeterol in the treatment of asthma. European Respiratory Review. 1995; 5(27):133-7.

GlaxoSmithKline 2005 [published data only]. GlaxoSmithKline. A double-blind evaluation of efficacy, safety, and tolerability of several single doses of salmeterol hydroxynaphthoate (GR33343G) compared with albuterol and placebo in asthmatic patients. GlaxoSmithKline Clinical Trial Register. 2005

Gongora 1991 [published data only]. Gongora HC, Wisniewski AF, Tattersfield AE. A single-dose comparison of inhaled albuterol and two formulations of salmeterol on airway reactivity in 
asthmatic subjects. American Review of Respiratory Disease. 1991; 144(3 Pt 1):626-9. [PubMed: 1679981]

Gotz 1995 [published data only]. Gotz MH. The efficacy and safety of inhaled salmeterol xinafoate (50 mcg bd) compared with salbutamol (200 mcg prn) in children with asthma. European Respiratory Journal. 1995; 8(Suppl 19):517S.

Gustafsson 1994 [published data only]. Gustafsson PM, Von BA, Jenkins MM. Salmeterol 50 mcg twice daily in the treatment of mild-to-moderate asthma in childhood - a comparison of two inhalation devices. European Journal of Clinical Research. 1994; 5:63-73.

Harper 2001 [published data only]. Harper TB, Stevens A, Clements D, Vandermeer A, Reisner C. Cardiac safety of salmeterol in 2 to 4 year old subjects with asthma. Journal of Allergy and Clinical Immunology. 2001; 107(2):S108.

Hermansson 1995 [published data only]. Hermansson BA, Jenkins RJ. A 4-week comparison of salmeterol and terbutaline in adult asthma. Allergy. 1995; 50(7):551-8. [PubMed: 8588686]

Inoue 2007 [published data only]. Inoue H, Komori M, Matsumoto T, Fukuyama S, Matsumura M, Nakano T, et al. Effects of salmeterol in patients with persistent asthma receiving inhaled corticosteroid plus theophylline. Respiration. 2007; Vol. 74(issue 6):611-6. [PubMed: 16960440]

Jartti 1998 [published data only]. Jartti TT, Kaila TJ, Tahvanainen KU, Kuusela TA, Vanto TT, Valimaki IA. Altered cardiovascular autonomic regulation after salmeterol treatment in asthmatic children. Clinical Physiology. 1998; 18(4):345-53. [PubMed: 9715761]

Jenkins 1991 [published data only]. Jenkins MM, Hilton CJ, De Kock JC, Palmer JB. Exacerbations of asthma in patients on salmeterol. Lancet. 1991; 337(8746):913-4. [PubMed: 1672988]

Johnson 1994 [published data only]. Johnson ME. A multicentre study to compare the efficacy and safety of salmeterol xinafoate and nedocromil sodium via metered-dose inhalers in adults with mild-to-moderate asthma. European Journal of Clinical Research. 1994; 5:75-85.

Kemp 1993 [published data only]. Kemp JP, Bierman CW, Cocchetto DM. Dose-response study of inhaled salmeterol in asthmatic patients with 24-hour spirometry and Holter monitoring. Annals of Allergy. 1993; 70(4):316-22. [PubMed: 8466097]

Kirby 1995 [published data only]. Kirby SM, Smith J, Ventresca GP. Salmeterol inhaler using a nonchlorinated propellant, HFA134a: systemic pharmacodynamic activity in healthy volunteers. Thorax. 1995; 50(6):679-81. [PubMed: 7638815]

Kraemer 1997 [published data only]. Kraemer R, Dhank B. Long term efficacy and tolerability of salmeterol $(50 \mu \mathrm{G} \mathrm{BD})$ administered by pressurised inhaler propelled by propellants 11 and 12 or by an alternative propellant, GR106642X for a period of 12 months in children with reversible airways obstruction. European Respiratory Journal. 1997; 10(Suppl 25):222S.

Kurihara 1993 [published data only]. Kurihara K, Mikawa H, Baba M, Ichikawa K, Masuda K, Yamada T, et al. Clinical study of salmeterol xinafoate (SN408) dry powder: comparative study between salmeterol xinafoate aerosol in children with bronchial asthma. Rinsho Iyaku (Journal of Clinical Therapeutics and Medicines). 1993; 9(10):2473-87.

Langley 1998 [published data only]. Langley SJ, Masterson CM, Batty EP, Woodcock A. Bronchodilator response to salbutamol after chronic dosing with salmeterol or placebo. European Respiratory Journal. 1998; 11(5):1081-5. [PubMed: 9648959]

Langton Hewer 1995 [published data only]. Langton Hewer S, Hobbs J, French D, Lenney W. Pilgrims progress: the effect of salmeterol in older children with chronic severe asthma. Respiratory Medicine. 1995; 89:435-40. [PubMed: 7644775]

Lemaigre 2006 [published data only]. Lemaigre V, Van den Bergh O, Smets A, De Peuter S, Verleden GM. Effects of long-acting bronchodilators and placebo on histamine-induced asthma symptoms and mild bronchus obstruction. Respiratory Medicine. 2006; 100(2):348-53. [PubMed: 15994073]

Lemanske 2001 [published data only]. Lemanske RF Jr, Sorkness CA, Mauger EA, Lazarus SC, Boushey HA, Fahy JV, et al. Inhaled corticosteroid reduction and elimination in patients with persistent asthma receiving salmeterol: a randomized controlled trial. JAMA. 2001; 285(20): 2594-603. [PubMed: 11368733] 
Lotvall 1998 [published data only]. Lotvall J, Lunde H, Svedmyr N. Onset of bronchodilation and finger tremor induced by salmeterol and salbutamol in asthmatic patients. Japanese Journal of Urology. 1998; 89(1):43-9. [PubMed: 9493421]

Lurie 2005 [published data only]. Lurie P, Wolfe SM. Misleading data analyses in salmeterol (SMART) study. Lancet. 2005; 366(9493):1261-2. [PubMed: 16214589]

Martin 1999 [published data only]. Martin RJ, Kraft M, Beaucher WN, Kiechel F, Sublett JL, LaVallee N, et al. Comparative study of extended release albuterol sulfate and long-acting inhaled salmeterol xinafoate in the treatment of nocturnal asthma. Annals of Allergy, Asthma, \& Immunology. 1999; 83(2):121-6.

Meier 1997 [published data only]. Meier CR, Jick H. Drug use and pulmonary death rates in increasingly symptomatic asthma patients in the UK. Thorax. 1997; 52(7):612-7. [PubMed: 9246132]

Mikawa 1993 [published data only]. Mikawa H, Mayumi M, Kimata H, Baba M, Ichikawa K, Matsumoto S, et al. Clinical study of salmeterol xinafoate (SN408) aerosol: comparative study by multiple administration in children with bronchial asthma. Rinsho Iyaku (Journal of Clinical Therapeutics and Medicines). 1993; 9(Suppl 4):179-99.

Miyamoto 1993a [published data only]. Miyamoto T, Takishima T, Makino S, Kabe J, Takahashi T, Yamakido M, et al. Clinical study of salmeterol xinafoate (SN408) aerosol: double-blind parallel study between procaterol tablet in patients with bronchial asthma. Rinsho Iyaku (Journal of Clinical Therapeutics and Medicines). 1993; 9(11):2671-99.

Miyamoto 1993b [published data only]. Miyamoto T, Takishima T, Makino S, Kabe J, Takahashi T, Yamakido M, et al. Clinical study of salmeterol xinafoate (SN408) aerosol: examination on optimal dose by multiple administration in patients with bronchial asthma. Rinsho Iyaku (Journal of Clinical Therapeutics and Medicines). 1993; 9(Suppl 4):81-111.

Miyamoto 1993c [published data only]. Miyamoto T, Takahashi T, Kabe J, Makino S. Clinical study of salmeterol xinafoate (SN408) aerosol: examination on bronchodilation by single administration in patients with bronchial asthma: examination on bronchodilation by single administration in patients with bronchial asthma. Rinsho Iyaku (Journal of Clinical Therapeutics and Medicines). 1993; 9(Suppl 4):23-48.

Miyamoto 1993d [published data only]. Miyamoto T, Takahashi T, Kabe J, Makino S. Clinical study of salmeterol xinafoate (SN408) dry powder: examination on bronchodilation by single administration in patients with bronchial asthma. Rinsho Iyaku (Journal of Clinical Therapeutics and Medicines). 1993; 9(Suppl 4):219-41.

Miyamoto 1993e [published data only]. Miyamoto T, Makino S, Kabe J, Takahashi T, Nakashima M. Clinical study of salmeterol xinafoate (SN408) aerosol: comparative study by multiple administration in patients with bronchial asthma. Rinsho Iyaku (Journal of Clinical Therapeutics and Medicines). 1993; 9:49-79.

Morgan 1994 [published data only]. Morgan, M. A comparison of the efficiency of salmeterol xinofoate with oral salbutamol controlled release against exercise induced asthma in adult asthmatics. National Research Register. 1994. https://portal.nihr.ac.uk/Pages/ NRRArchiveSearch.aspx[: N0123137694]

Muir 1992 [published data only]. Muir JF, Georges D. Usefulness of salmeterol in nocturnal asthma: a comparative study with theophylline-ketotifen association. A French multicenter group. Revue des Maladies Respiratoires. 1992; 9(Suppl 1):R23-6. [PubMed: 1350366]

Nathan 1995 [published data only]. Nathan RA, Seltzer JM, Kemp JP, Chervinsky P, Alexander WJ, Liddle R, et al. Safety of salmeterol in the maintenance treatment of asthma. Annals of Allergy, Asthma \& Immunology. 1995; 75(3):243-8.

Nelson 1999 [published data only]. Nelson HS, Berkowitz RB, Tinkelman DA, Emmett AH, Rickard KA, Yancey SW. Lack of subsensitivity to albuterol after treatment with salmeterol in patients with asthma. American Journal of Respiratory and Critical Care Medicine. 1999; 159(5 Pt 1): 1556-61. [PubMed: 10228126]

Nelson 2001 [published data only]. Nelson HS, Nathan RA, Kalberg C, Yancey SW, Rickard KA. Comparison of inhaled salmeterol and oral zafirlukast in asthmatic patients using concomitant inhaled corticosteroids. Medscape General Medicine. 2001; 3(4):3. [PubMed: 11549982] 
Nishima 1993 [published data only]. Nishima S, Odajima H, Origasa H. Clinical study of salmeterol xinafoate (SN408) dry powder: bioequivalency study between salmeterol xinafoate aerosol in children with bronchial asthma by single dose crossover method. Rinsho Iyaku (Journal of Clinical Therapeutics and Medicines). 1993; 9(9):2133-46.

Nishiyama 2006 [published data only]. Nishiyama O, Taniguchi H, Kondoh Y, Kimura T, Kato K, Kume H, et al. Comparison of the effects of tulobuterol patch and salmeterol in moderate to severe asthma. Clinical and Experimental Pharmacology and Physiology. 2006; 33(11):1016-21. [PubMed: 17042908]

Norhaya 1999 [published data only]. Norhaya MR, Yap TM, Zainudin BMZ. Addition of inhaled salmeterol to inhaled corticosteroids in patients with poorly controlled nocturnal asthma. Respirology. 1999; 4(1):77-81. [PubMed: 10339734]

Nutini 1998 [published data only]. Nutini S, Martini T, Righi R. Long-term treatment of asthmatic patients with salmeterol vs slow-release theophylline. Respiratory Medicine. 1998; 92(4):683-90. [PubMed: 9659537]

Orgel 1985 [published data only]. Orgel HA, Kemp JP, Tinkelman DG, Webb DR Jr. Bitolterol and albuterol metered-dose aerosols: comparison of two long-acting beta 2-adrenergic bronchodilators for treatment of asthma. Journal of Allergy and Clinical Immunology. 1985; 75(1 Pt 1):55-62. [PubMed: 3968329]

Ortiz 2002 [published data only]. Ortiz G, Menendez R. The effects of inhaled albuterol and salmeterol in 2- to 5-year-old asthmatic children as measured by impulse oscillometry. Journal of Asthma. 2002; 39(6):531-6. [PubMed: 12375713]

Paggiaro 1996 [published data only]. Paggiaro PL, Giannini D, Di FA, Testi R. Comparison of inhaled salmeterol and individually dose-titrated slow-release theophylline in patients with reversible airway obstruction. European Respiratory Journal. 1996; 9(8):1689-95. [PubMed: 8866596]

Palmer 1992 [published data only]. Palmer JB, Stuart AM, Shepherd GL, Viskum K. Inhaled salmeterol in the treatment of patients with moderate to severe reversible obstructive airways disease - a 3-month comparison of the efficacy and safety of twice-daily salmeterol 100 micrograms with salmeterol 50 micrograms. Respiratory Medicine. 1992; 86(5):409-17. [PubMed: 1361068]

Pascoe 2006 [published data only]. Pascoe S, Knowles J, Glasbrenner M, Duvauchelle T, Fuhr R, Brookman J. Efficacy and safety of therapeutic and supratherapeutic doses of indacaterol compared to salmeterol and salbutamol in mild to moderate asthma [Abstract]. European Respiratory Journal. 2006; Vol. 28(issue Suppl 50):207s. [P1238].

Pastorello 1998 [published data only]. Pastorello EA, Mauro M, Incorvaia C. Comparison of efficacy and safety of inhaled salmeterol and slow-release oral theophylline in patients with moderate/ severe asthma. Internista. 1998; 6(2):101-7.

Pearlman 1999 [published data only]. Pearlman DS, Stricker W, Weinstein S, Gross G, Chervinsky P, Woodring A, et al. Inhaled salmeterol and fluticasone: a study comparing monotherapy and combination therapy in asthma. Annals of Allergy, Asthma, \& Immunology. 1999; 82(3):257-65.

Peslis 1994 [published data only]. Peslis N, Weber HH, Kettner J. Salmeterol and fenoterol comparison of efficacy and safety in patients with reversible obstructive airways disease. European Respiratory Journal. 1994; 7(Suppl 18):112s.

Peters 2000 [published data only]. Peters JI, Shelledy DC, Jones AP Jr, Lawson RW, Davis CP, LeGrand TS. A randomized, placebo-controlled study to evaluate the role of salmeterol in the inhospital management of asthma. Chest. 2000; 118(2):313-20. [PubMed: 10936118]

Pohunek 2004 [published data only]. Pohunek P, Matulka M, Rybnicek O, Kopriva F, Honomichlova H, Svobodova T. Dose-related efficacy and safety of formoterol (Oxis) Turbuhaler compared with salmeterol Diskhaler in children with asthma. Pediatric Allergy and Immunology. 2004; 15(1):32-9. [PubMed: 14998380]

Pollard 1997 [published data only]. Pollard SJ, Spector SL, Yancey SW, Cox FM, Emmett A. Salmeterol versus theophylline in the treatment of asthma. Annals of Allergy, Asthma, \& Immunology. 1997; 78(5):457-64. 
Prieto 2002 [published data only]. Prieto L, Gutierrez V, Torres V, Uixera S, Marin J. Effect of salmeterol on seasonal changes in airway responsiveness and exhaled nitric oxide in pollensensitive asthmatic subjects. Chest. 2002; 122(3):798-805. [PubMed: 12226016]

Revill 1998 [published data only]. Revill SM, Morgan MD. The cardiorespiratory response to submaximal exercise in subjects with asthma following pretreatment with controlled release oral salbutamol and high-dose inhaled salmeterol. Respiratory Medicine. 1998; 92(8):1053-8. [PubMed: 9893775]

Rhee 1997 [published data only]. Rhee YK. A comparison of salmeterol with salbutamol inhalation in treatment of mild to moderate asthma. Tuberculosis and Respiratory Diseases. 1997; 44(4): 815-21.

Ringbaek 1996 [published data only]. Ringbaek TJ, Soes-Petersen U, Christensen M, Iversen ET, Rasmussen FV. Salmeterol improves the control of disease in patients with moderate asthma. A comparative study of inhaled salmeterol $50 \mathrm{mg}$ and salbutamol depot tablets $8 \mathrm{mg}$, both administered twice daily. Ugeskrift for Laeger. 1996; 158(27):3940-3. [PubMed: 8701511]

Ringdal 1995 [published data only]. Ringdal N, Bateman E, Laitinen L, Sykes AP. Efficacy and safety of salmeterol $50 \mathrm{mcg}$ via a pressurised inhaler formulated with GR106642X. American Journal of Respiratory and Critical Care Medicine. 1995; 151(4 Pt 2):A57.

Ritz 1997 [published data only]. Ritz M, Thorens JB, Arnold-Ketterer M, Chevrolet JC. Effects of inhaled salmeterol and salbutamol (albuterol) on morning dips compared in intensive care patients recovering from an acute severe asthma attack. Intensive Care Medicine. 1997; 23(12): 1225-30. [PubMed: 9470077]

Roberts 1999 [published data only]. Roberts JA, Bradding P, Britten KM, Walls AF, Wilson S, Gratziou C, et al. The long-acting beta2-agonist salmeterol xinafoate: effects on airway inflammation in asthma. European Respiratory Journal. 1999; 14(2):275-82. [PubMed: 10515401]

Sano 1993 [published data only]. Sano Y, Miyamoto Y, Arai Y, Tanaka Y, Akiyama N, Origasa H. Clinical study of salmeterol xinafoate (SN408) dry powder: bioequivalency study between salmeterol xinafoate aerosol in patients with bronchial asthma by single dose crossover method. Rinsho Iyaku (Journal of Clinical Therapeutics and Medicines). 1993; 9(9):2105-18.

Schaanning 1996 [published data only]. Schaanning J, Vilsvik J, Henriksen AH, Bratten G. Efficacy and duration of salmeterol powder inhalation in protecting against exercise-induced bronchoconstriction. Annals of Allergy, Asthma, \& Immunology. 1996; 76(1):57-60.

Shaheen 1994 [published data only]. Shaheen MZ, Ayres JG, Benincasa C. Incidence of acute decreases in peak expiratory flow following the use of metered-dose inhalers in asthmatic patients. European Respiratory Journal. 1994; 7(12):2160-4. [PubMed: 7713197]

Shepherd 1991 [published data only]. Shepherd GL, Jenkins WJ, Alexander J. Asthma exacerbations in patients taking regular salmeterol, or salbutamol for symptoms. Lancet. 1991; 337(8754):1424. [PubMed: 1674807]

SLGL24 [published data only]. * SLGL24. A double-blind, randomised, parallel group study to investigate the efficacy of inhaled salmeterol $50 \mathrm{mcg}$ administered twice daily compared with salbutamol $200 \mathrm{mcg}$ administered four times daily in patients with poorly controlled nocturnal asthma. http://ctr.gsk.co.uk/Summary/salmeterol/studylist.asp

Stahl 1999 [published data only]. Stahl E, Svensson K, Crompton GK. Bambuterol is a more costeffective treatment strategy than salmeterol in asthmatic patients with nocturnal symptoms. Journal of Medical Economics. 1999; 2:117-22.

Starke 1996 [published data only]. Starke ID, Luce P. The efficacy and safety of inhaled salmeterol 50 microg bd in older patients with reversible airflow obstruction. Age and Ageing. 1996; 25(1): 67-71. [PubMed: 8670532]

Storms 2004 [published data only]. Storms W, Chervinsky P, Ghannam AF, Bird S, Hustad CM, Edelman JM, et al. A comparison of the effects of oral montelukast and inhaled salmeterol on response to rescue bronchodilation after challenge. Respiratory Medicine. 2004; 98(11):1051-62. [PubMed: 15526805]

Szczeklik 1998 [published data only]. Szczeklik A, Dworski R, Mastalerz L, Prokop A, Sheller JR, Nizankowska E, et al. Salmeterol prevents aspirin-induced attacks of asthma and interferes with 
eicosanoid metabolism. American Journal of Respiratory and Critical Care Medicine. 1998; 158(4):1168-72. [PubMed: 9769277]

Taguchi 1993 [published data only]. Taguchi O, Ibata H, Suzuki S, Miyamoto T. Clinical study of salmeterol xinafoate (SN408) in a regular inhalation therapy in patients with bronchial asthma: comparative evaluation with azelastine regarding its effect on bronchial hyperresponsiveness. Rinsho Iyaku (Journal of Clinical Therapeutics and Medicines). 1993; 9(11):2711-23.

Taylor 1992 [published data only]. Taylor IK, O’Shaughnessy KM, Choudry NB, Adachi M, Palmer JB, Fuller RW. A comparative study in atopic subjects with asthma of the effects of salmeterol and salbutamol on allergen-induced bronchoconstriction, increase in airway reactivity, and increase in urinary leukotriene E4 excretion. Journal of Allergy \& Clinical Immunology. 1992; 89(2):575-83. [PubMed: 1346794]

Taylor 1998 [published data only]. Taylor DR, Town GI, Herbison GP, Boothman-Burrell D, Flannery EM, Hancox B, et al. Asthma control during long-term treatment with regular inhaled salbutamol and salmeterol published [erratum appears in Thorax 1999 Feb; 542:188]. Thorax. 1998; 53(9):744-52. [PubMed: 10319056]

Taylor 2000a [published data only]. Taylor DR, Drazen JM, Herbison GP, Yandava CN, Hancox RJ, Town GI. Asthma exacerbations during long term beta agonist use: influence of beta2 adrenoceptor polymorphism. Thorax. 2000; 55(9):762-7. [PubMed: 10950895]

Taylor 2000b [published data only]. Taylor DR, Hancox RJ, McRae W, Cowan JO, Flannery EM, McLachlan CR, et al. The influence of polymorphism at position 16 of the beta2-adrenoceptor on the development of tolerance to beta-agonist. Journal of Asthma. 2000; 37(8):691-700. [PubMed: 11192234]

Thompson 1994 [published data only]. Thompson P, Bellesis M. Long-term safety and efficacy of salmeterol in nocturnal asthmatics. Australian \& New Zealand Journal of Medicine. 1994; 24:459.

Tomac 1996 [published data only]. Tomac N, Tuncer A, Saraclar Y, Adalioglu G. Efficacy of salmeterol in the treatment of childhood asthma. Acta Paediatrica Japonica. 1996; 38(5):489-94. [PubMed: 8942009]

Ukena 1997 [published data only]. Ukena D, Koper I, Braun H, Leutz M, Schlimmer P, Sybrecht GW. Therapy of nocturnal asthma: salmeterol versus nocturnal administration of retard theophylline--comparison of effectiveness and tolerance. Pneumologie. 1997; 51(3):317-23. [PubMed: 9173422]

Ullman 1990 [published data only]. Ullman A, Hedner J, Svedmyr N. Inhaled salmeterol and salbutamol in asthmatic patients. An evaluation of asthma symptoms and the possible development of tachyphylaxis. American Review of Respiratory Disease. 1990; 142(3):571-5. [PubMed: 1975162]

Venables 1992 [published data only]. Venables T. A multicentre study in general practice to compare the efficacy and tolerability of inhaled salmeterol xinafoate and terbutaline in the treatment of asthma. British Journal of Clinical Research. 1992; 3:125-36.

Verberne 1993 [published data only]. Verberne AA, Hop WC, Bos AB, Kerrebijn KF. Effect of a single dose of inhaled salmeterol on baseline airway caliber and methacholine-induced airway obstruction in asthmatic children. Journal of Allergy and Clinical Immunology. 1993; 91(1 Pt 1): 127-34. [PubMed: 8093705]

Verberne 1998 [published data only]. * SLGB4014 (SLPT15). Placebo controlled study during one year comparing the addition of salmeterol with an increase of the dose of the inhaled corticosteroid in asthmatic children already on treatment with inhaled corticosteroids. http:// ctr.gsk.co.uk/Summary/salmeterol/IV.SLGB4014.pdfVerberne AA, Frost C, Roorda RJ, Van der Laag H, Kerrebijn KF. One year treatment with salmeterol compared with beclomethasone in children with asthma. The Dutch Paediatric Asthma Study Group. American Journal of Respiratory \& Critical Care Medicine. 1997; 156(3 Pt 1):688-95. [PubMed: 9309980]

Verini 1998 [published data only]. Verini M, Verrotti A, Greco R, Chiarelli F. Comparison of the bronchodilator effect of inhaled short- and long-acting beta2-agonists in children with bronchial asthma. A randomised trial. Clinical Drug Investigation. 1998; 16(1):19-24. [PubMed: 18370514] 
Villaran 1999 [published data only]. Villaran C, O’Neill SJ, Helbling A, Van Noord JA, Lee TH, Chuchalin AG, et al. Montelukast versus salmeterol in patients with asthma and exercise-induced bronchoconstriction. Montelukast/Salmeterol Exercise Study Group. Journal of Allergy \& Clinical Immunology. 1999; 104(3 Pt 1):547-53. [PubMed: 10482826]

Weersink 1997 [published data only]. Weersink EJ, Van Zomeren EH, Koeter GH, Postma DS. Treatment of nocturnal airway obstruction improves daytime cognitive performance in asthmatics. American Journal of Respiratory \& Critical Care Medicine. 1997; 156(4 Pt 1):114450. [PubMed: 9351614]

Weiner 2003 [published data only]. Weiner P, Magadle R, Beckerman M, Berar-Yanay N. The effect of time on the perception of dyspnea following inhaled long-acting bronchodilator. Harefuah. 2003; 142(5):342-4. 398. [PubMed: 12803056]

Weinstein 1997 [published data only]. Weinstein S, Chervinsky P, Pollard SJ, Bronsky EA, Nathan RA, Prenner B, et al. A one-week dose-ranging study of inhaled salmeterol in children with asthma. Journal of Asthma. 1997; 34(1):43-52. [PubMed: 9033439]

Wiegand 1999 [published data only]. Wiegand L, Mende CN, Zaidel G, Zwillich CW, Petrocella VJ, Yancey SW, et al. Salmeterol vs theophylline: sleep and efficacy outcomes in patients with nocturnal asthma. Chest. 1999; 115(6):1525-32. [PubMed: 10378544]

Wilding 1997 [published data only]. Wilding P, Clark M, Coon JT, Lewis S, Rushton L, Bennett J, et al. Effect of long term treatment with salmeterol on asthma control: A double blind, randomised crossover study. BMJ. 1997; 314(7092):1441-6. [PubMed: 9167559]

Williams 1998 [published data only]. Williams C, Crossland L, Finnerty J, Crane J, Holgate S, Pearce N, et al. Case-control study of salmeterol and near-fatal attacks of asthma. Thorax. 1998; 53(1):7-13. [PubMed: 9577515]

Zarkovic 1998 [published data only]. Zarkovic J, Gotz MH, Holgate ST, Taak NK. Effect of longterm regular salmeterol treatment in children with moderate asthma. Clinical Drug Investigation. 1998; 15(3):169-175.

Zimmermann 2003 [published data only]. Zimmermann T, Gulyas A, Bauer CP, Steinkamp G, Trautmann M. Salmeterol versus sodium cromoglycate for the protection of exercise induced asthma in children--a randomised cross-over study. European Journal of Medical Research. 2003; 8(9):428-34. [PubMed: 14555299]

\section{Additional references}

Altman 2003. Altman DG, Bland JM. Statistics notes: interaction revisited: the difference between two estimates. BMJ. 2003; 326(7382):219. [PubMed: 12543843]

Anderson 2006. Anderson GP. Current issues with beta(2)-adrenoceptor agonists - pharmacology and molecular and cellular mechanisms. Clinical Reviews in Allergy \& Immunology. 2006; Vol. 31(issue 2-3):119-30. [PubMed: 17085788]

Arnold 1985. Arnold JMO, Oconnor PC, Riddell JG, Harron DWG, Shanks RG, McDevitt DG. Effects of the beta-2-adrenoceptor antagonist ici-118,551 on exercise tachycardia and isoprenalineinduced beta-adrenoceptor responses in man. British Journal of Clinical Pharmacology. 1985; Vol. 19(issue 5):619-30. [PubMed: 2860915]

Barnes 1993. Barnes PJ. Beta-adrenoceptors on smooth-muscle, nerves and inflammatory cells. Life Sciences. 1993; Vol. 52(issue 26):2101-9. [PubMed: 8389953]

Barnes 1995. Barnes PJ. Beta-adrenergic receptors and their regulation. American Journal of Respiratory and Critical Care Medicine. 1995; Vol. 152(issue 3):838-60. [PubMed: 7663795]

Bennett 1994. Bennett JA, Smyth ET, Pavord ID, Wilding PJ, Tattersfield AE. Systemic effects of salbutamol and salmeterol in patients with asthma. Thorax. 1994; Vol. 49(issue 8):771-4. [PubMed: 8091321]

Benson 1948. Benson RL, Perlman F. Clinical effects of epinephrine by inhalation - a survey. Journal of Allergy. 1948; Vol. 19(issue 2):129-40. [PubMed: 18911788]

Bergman 1969. Bergman J, Persson H, Wetterli K. Two new groups of selective stimulants of adrenergic beta-receptors. Experientia. 1969; Vol. 25(issue 9):899-901. [PubMed: 4391906] 
Bijl-Hofland 2001. Bijl-Hofland ID, Cloosterman SG, Folgering HT, Van den Elshout FJ, Van Weel C, Van Schayck CP. Inhaled corticosteroids, combined with long-acting beta(2)-agonists, improve the perception of bronchoconstriction in asthma. American Journal of Respiratory and Critical Care Medicine. 2001; Vol. 164(issue 5):764-9. [PubMed: 11549530]

Boushey 1980. Boushey HA, Holtzman MJ, Sheller JR, Nadel JA. Bronchial hyperreactivity. American Review of Respiratory Disease. 1980; Vol. 121(issue 2):389-413. [0003-0805: (Print)]. [PubMed: 6987924]

Bousquet 1990. Bousquet J, Chanez P, Lacoste JY, Barneon G, Ghavanian N, Enander I, et al. Eosinophilic inflammation in asthma. New England Journal of Medicine. 1990; Vol. 323(issue 15):1033-9. [PubMed: 2215562]

Bousquet 2000. Bousquet J, Jeffery PK, Busse WW, Johnson M, Vignola AM. Asthma. From bronchoconstriction to airways inflammation and remodeling. American Journal Respiratory Critical Care Medicine. 2000; Vol. 161(issue 5):1720-45.

Brewster 1990. Brewster CEP, Howarth PH, Djukanovic R, Wilson J, Holgate ST, Roche WR. Myofibroblasts and subepithelial fibrosis in bronchial-asthma. American Journal of Respiratory Cell and Molecular Biology. 1990; Vol. 3(issue 5):507-11. [PubMed: 2223105]

Brown 1983. Brown MJ, Brown DC, Murphy MB. Hypokalemia from beta-2-receptor stimulation by circulating epinephrine. New England Journal of Medicine. 1983; Vol. 309(issue 23):1414-9. [PubMed: 6314140]

Brusasco 1998. Brusasco V, Crimi E, Pellegrino R. Airway hyperresponsiveness in asthma: not just a matter of airway inflammation. Thorax. 1998; Vol. 53(issue 11):992-8. [PubMed: 10193402]

Burgess 1991. Burgess CD, Windom HH, Pearce N, Marshall S, Beasley R, Siebers RWL, et al. Lack of evidence for beta-2 receptor selectivity - a study of metaproterenol, fenoterol, isoproterenol, and epinephrine in patients with asthma. American Review of Respiratory Disease. 1991; Vol. 143(issue 2):444-6. [PubMed: 1671326]

Burggraaf 2001. Burggraaf J, Westendorp RGJ, In't Veen J, Schoemaker RC, Sterk PJ, Cohen AF, et al. Cardiovascular side effects of inhaled salbutamol in hypoxic asthmatic patients. Thorax. 2001; Vol. 56(issue 7):567-9. [PubMed: 11413357]

Carroll 1993. Carroll N, Elliot J, Morton A, James A. The structure of large and small airways in nonfatal and fatal asthma. American Review of Respiratory Disease. 1993; Vol. 147(issue 2): 405-10. [PubMed: 8430966]

Cates 2009. Cates CJ, Lasserson TJ, Jaeschke R. Regular treatment with salmeterol and inhaled steroids for chronic asthma: serious adverse events. Cochrane Database of Systematic Reviews. 2009; (Issue 3) [DOI: 10.1002/14651858.CD006922.pub2].

Cockcroft 1993. Cockcroft DW, McParland CP, Britto SA, Swystun VA, Rutherford BC. Regular inhaled salbutamol and airway responsiveness to allergen. Lancet. 1993; Vol. 342(issue 8875): 833-7. [PubMed: 8104272]

Cockcroft 2006. Cockcroft D. Airway hyperresponsiveness as a determinant of the early asthmatic response to inhaled allergen. Journal of Asthma. 2006; Vol. 43(issue 3):175-8. [PubMed: 16754517]

Collins 1969. Collins JM, McDevitt DG, Shanks RG, Swanton JG. Cardio-toxicity of isoprenaline during hypoxia. British Journal of Pharmacology. 1969; 36(1):36-45.

Crane 1989. Crane J, Pearce N, Flatt A, Burgess C, Jackson R, Kwong T, et al. Prescribed fenoterol and death from asthma in New Zealand, 1981-83 - case-control study. Lancet. 1989; Vol. 1(issue 8644):917-22. [PubMed: 2565417]

Cullum 1969. Cullum VA, Farmer JB, Jack D, Levy GP. Salbutamol - a new selective betaadrenoceptive receptor stimulant. British Journal of Pharmacology. 1969; 35(1):141-51. [PubMed: 5762037]

Delea 2008. Delea TE, Hagiwara M, Stanford RH, Stempel DA. Effects of fluticasone propionate/ salmeterol combination on asthma-related health care resource utilization and costs and adherence in children and adults with asthma. Clinical Therapeutics. 2008; 30(3):560-71. [PubMed: 18405796]

Ducharme 2006. Ducharme FM, Lasserson TJ, Cates CJ. Long-acting beta-2 agonists versus antileukotrienes as add-on therapy to inhaled corticosteroids for chronic asthma (Cochrane Review). 
Cochrane Database of Systematic Reviews. 2006; (Issue 4) [DOI:

10.1002/14651858.CD003137.pub3].

Dunnill 1960. Dunnill MS. The pathology of asthma, with special reference to changes in the bronchial mucosa. Journal of Clinical Pathology. 1960; Vol. 13(issue 1):27-33. [PubMed: 13818688]

Ebina 1993. Ebina M, Takahashi T, Chiba T, Motomiya M. Cellular hypertrophy and hyperplasia of airway smooth muscles underlying bronchial-asthma - a 3-d morphometric studyY. American Review of Respiratory Disease. 1993; Vol. 148(issue 3):720-6. [PubMed: 8368645]

Gay 1949. Gay LN, Long JW. Clinical evaluation of isopropylepinephrine in management of bronchial asthma. Journal of the American Medical Association. 1949; Vol. 139(issue 7):452-7. [PubMed: 18107961]

Grainger 1991. Grainger J, Woodman K, Pearce N, Crane J, Burgess C, Keane A, et al. Prescribed fenoterol and death from asthma in New Zealand, 1981-7 - a further case-control study. Thorax. 1991; Vol. 46(issue 2):105-11. [PubMed: 2014490]

Greenstone 2005. Greenstone IR, Ni Chroinin M, Masse V, Danish A, Magdalinos H, Zhang X, et al. Combination of inhaled long-acting beta2-agonists and inhaled steroids versus higher dose of inhaled steroids in children and adults with persistent asthma (Cochrane Review). Cochrane Database of Systematic Reviews. 2005; (Issue 4) [DOI: 10.1002/14651858.CD005533].

Guhan 2000. Guhan AR, Cooper S, Oborne J, Lewis S, Bennett J, Tattersfield AE. Systemic effects of formoterol and salmeterol: a dose-response comparison in healthy subjects. Thorax. 2000; Vol. 55(issue 8):650-6. [PubMed: 10899240]

Haley 1998. Haley KJ, Drazen JM. Inflammation and airway function in asthma - what you see is not necessarily what you get. American Journal of Respiratory and Critical Care Medicine. 1998; Vol. 157(issue 1):1-3. [PubMed: 9445269]

Hall 1989. Hall JA, Petch MC, Brown MJ. Intracoronary injections of salbutamol demonstrate the presence of functional beta-2-adrenoceptors in the human-heart. Circulation Research. 1989; Vol. 65(issue 3):546-53. [PubMed: 2548759]

Hanania 2002. Hanania NA, Sharfkhaneh A, Barber R, Dickey BF. beta-agonist intrinsic efficacy measurement and clinical significance. American Journal of Respiratory and Critical Care Medicine. 2002; Vol. 165(issue 10):1353-8. [PubMed: 12016095]

Hanania 2007. Hanania NA, Moore RH, Zimmerman JL, Miller CT, Bag R, Sharafkhaneh A, et al. The role of intrinsic efficacy in determining response to beta(2)-agonist in acute severe asthma. Respiratory Medicine. 2007; Vol. 101(issue 5):1007-14. [PubMed: 17052901]

Hancox 1999. Hancox RJ, Aldridge RE, Cowan JO, Flannery EM, Herbison GP, McLachlan CR, et al. Tolerance to beta-agonists during acute bronchoconstriction. European Respiratory Journal. 1999; Vol. 14(issue 2):283-7. [PubMed: 10515402]

Hancox 2006a. Hancox RJ. Concluding remarks: can we explain the association of beta-agonists with asthma mortality? A hypothesis. Clinical Reviews in Allergy and Immunology. 2006; Vol. 31(issue 2-3):279-88. [PubMed: 17085800]

Hancox 2006b. Hancox RJ. Interactions between corticosteroids and beta2-agonists. Clinical Reviews in Allergy and Immunology. 2006; Vol. 31(issue 2-3):231-46. [PubMed: 17085796]

Haney 2006. Haney S, Hancox RJ. Recovery from bronchoconstriction and bronchodilator tolerance. Clinical Reviews in Allergy \& Immunology. 2006; Vol. 31(issue 2-3):181-96. [PubMed: 17085792]

Harvey 1982. Harvey JE, Tattersfield AE. Airway response to salbutamol - effect of regular salbutamol inhalations in normal, atopic, and asthmatic subjects. Thorax. 1982; Vol. 37(issue 4): 280-7. [PubMed: 7112457]

Higgins 2008. Higgins, JPT.; Green, S., editors. Cochrane Handbook for Systematic Reviews of Interventions. Version 5.0.0. The Cochrane Collaboration; 2008. Available from www.cochranehandbook.org [updated February 2008]

Jones 2001. Jones SL, Cowan JO, Flannery EM, Hancox RJ, Herbison GP, Taylor DR. Reversing acute bronchoconstriction in asthma: the effect of bronchodilator tolerance after treatment with formoterol. European Respiratory Journal. 2001; Vol. 17(issue 3):368-73. [PubMed: 11405513]

Konzett 1940. Konzett H. Neue broncholytisch hochwirksame Körper der Adrenalinreihe. NaunynSchmiedeberg's Archives of Pharmacology. 1940; Vol. 197:27-40. 
Kuyper 2003. Kuyper LM, Pare PD, Hogg JC, Lambert RK, Ionescu D, Woods R, et al. Characterization of airway plugging in fatal asthma. American Journal of Medicine. 2003; Vol. 115(issue 1):6-11. [PubMed: 12867228]

Lee 2003. Lee DKC, Jackson CM, Currie GP, Cockburn WJ, Lipworth BJ. Comparison of combination inhalers vs inhaled corticosteroids alone in moderate persistent asthma. British Journal of Clinical Pharmacology. 2003; Vol. 56(issue 5):494-500. [PubMed: 14651722]

Lipworth 1989. Lipworth BJ, Struthers AD, McDevitt DG. Tachyphylaxis to systemic but not to airway responses during prolonged therapy with high-dose inhaled salbutamol in asthmatics. American Review of Respiratory Disease. 1989; Vol. 140(issue 3):586-92. [PubMed: 2782734]

Lipworth 1992. Lipworth BJ, McDevitt DG. Inhaled beta-2-adrenoceptor agonists in asthma - help or hindrance. British Journal of Clinical Pharmacology. 1992; Vol. 33(issue 2):129-38. [PubMed: 1347999]

Lipworth 1997. Lipworth BJ. Airway subsensitivity with long-acting beta 2-agonists: is there cause for concern? Drug Safety. 1997; 16(5):295-308. [PubMed: 9187530]

Lipworth 2000. Lipworth BJ, Aziz I. Bronchodilator response to albuterol after regular formoterol and effects of acute corticosteroid administration. Chest. 2000; Vol. 117(issue 1):156-62. [PubMed: 10631214]

Martinez 2006. Martinez FD. Serious adverse events and death associated with treatment using longacting beta-agonists. Clinical Reviews in Allergy \& Immunology. 2006; Vol. 31(issue 2-3):26978. [PubMed: 17085799]

McDevitt 1974. McDevitt DG, Shanks RG, Swanton JG. Further observations on cardiotoxicity of isoprenaline during hypoxia. British Journal of Pharmacology. 1974; Vol. 50(issue 3):335-44. [PubMed: 4852115]

McIvor 1998. McIvor RA, Pizzichini E, Turner MO, Hussack P, Hargreave FE, Sears MR. Potential masking effects of salmeterol on airway inflammation in asthma. American Journal of Respiratory and Critical Care Medicine. 1998; Vol. 158(issue 3):924-30. [PubMed: 9731027]

Miranda 2004. Miranda C, Busacker A, Balzar S, Trudeau J, Wenzel SE. Distinguishing severe asthma phenotypes: role of age at onset and eosinophilic inflammation. Journal of Allergy and Clinical Immunology. 2004; Vol. 113(issue 1):101-8. [PubMed: 14713914]

Morrison 1993. Morrison KJ, Gao Y, Vanhoutte PM. Beta-adrenoceptors and the epithelial layer in airways. Life Sciences. 1993; Vol. 52(issue 26):2123-30. [PubMed: 8389954]

Nelson 1977. Nelson HS, Raine D, Doner HC, Posey WC. Subsensitivity to bronchodilator action of albuterol produced by chronic administration. American Review of Respiratory Disease. 1977; Vol. 116(issue 5):871-8. [PubMed: 335936]

Ni Chroinin 2004. Ni Chroinin M, Greenstone IR, Ducharme FM. Addition of inhaled long-acting beta2-agonists to inhaled steroids as first line therapy for persistent asthma in steroid-naive adults. Cochrane Database of Systematic Reviews. 2004; Issue 4 [DOI: 10.1002/14651858.CD005307].

Ni Chroinin 2005. Ni Chroinin M, Greenstone IR, Danish A, Magdolinos H, Masse V, Zhang X, et al. Long-acting beta2-agonists versus placebo in addition to inhaled corticosteroids in children and adults with chronic asthma. Cochrane Database of Systematic Reviews. 2005; (Issue 4) [DOI: 10.1002/14651858.CD005535].

Ordonez 2001. Ordonez CL, Khashayar R, Wong HH, Ferrando RON, Wu R, Hyde DM, et al. Mild and moderate asthma is associated with airway goblet cell hyperplasia and abnormalities in mucin gene expression. American Journal of Respiratory and Critical Care Medicine. 2001; Vol. 163(issue 2):517-23. [PubMed: 11179133]

Palmqvist 1999. Palmqvist M, Ibsen T, Mellen A, Lotvall J. Comparison of the relative efficacy of formoterol and salmeterol in asthmatic patients. American Journal of Respiratory and Critical Care Medicine. 1999; Vol. 160(issue 1):244-9. [PubMed: 10390407]

Pearce 1990. Pearce N, Grainger J, Atkinson M, Crane J, Burgess C, Culling C, et al. Case-control study of prescribed fenoterol and death from asthma in New Zealand, 1977-81. Thorax. 1990; Vol. 45(issue 3):170-5. [PubMed: 2330548]

Pearce 2001. Pearce, NBR.; Crane, J.; Burgess, C. Asthma and Rhinitis. Vol. 2001. Blackwell Scientific; Oxford: 2001. Epidemiology of asthma mortality; p. 56-69. 
Pearce 2007. Pearce, N. Adverse Reactions: The Fenoterol Story. 1st Edition. Auckland University Press; Auckland: 2007. p. 215

Phillips 1990. Phillips GD, Finnerty JP, Holgate ST. Comparative protective effect of the inhaled beta-2-agonist salbutamol (albuterol) on bronchoconstriction provoked by histamine, methacholine, and adenosine $5^{\prime}$-monophosphate in asthma. Journal of Allergy and Clinical Immunology. 1990; Vol. 85(issue 4):755-62. [PubMed: 2182697]

RevMan 2011. The Nordic Cochrane Centre. The Cochrane Collaboration. Review Manager (RevMan). 5.1. The Nordic Cochrane Centre, The Cochrane Collaboration; Copenhagen: 2011.

Ringdal 1998. Ringdal N, Derom E, Wahlin-Boll E, Pauwels R. Onset and duration of action of single doses of formoterol inhaled via Turbuhaler (R). Respiratory Medicine. 1998; Vol. 92(issue 8): 1017-21. [PubMed: 9893769]

Salpeter 2006. Salpeter SR, Buckley NS, Ormiston TM, Salpeter EE. Meta-analysis: effect of longacting beta-agonists on severe asthma exacerbations and asthma-related deaths. Annals of Internal Medicine. 2006; 144(12):904-12. [PubMed: 16754916]

Sears 1990. Sears MR, Taylor DR, Print CG, Lake DC, Li QQ, Flannery EM, et al. Regular inhaled beta-agonist treatment in bronchial-asthma. Lancet. 1990; Vol. 336(issue 8728):1391-6. [PubMed: 1978871]

Simonsson 1967. Simonsso BG, Jacobs FM, Nadel JA. Role of autonomic nervous system and the cough reflex in the increased responsiveness of airways in patients with obstructive airway disease. Journal of Clinical Investigation. 1967; Vol. 46(issue 11):1812-8.

Sovani 2008. Sovani MP, Whale CI, Oborne J, Cooper S, Mortimer K, Ekström T, et al. Poor adherence with inhaled corticosteroids for asthma: can using a single inhaler containing budesonide and formoterol help? British Journal of General Practice. 2008; Vol. 58:37-43. [PubMed: 18186995]

Speizer 1968. Speizer FE, Doll R, Heaf P. Observations on recent increase in mortality from asthma. British Medical Journal. 1968; Vol. 1(issue 5588):335-9. [PubMed: 5317525]

Stolley 1972. Stolley PD. Asthma mortality. Why the United States was spared an epidemic of deaths due to asthma. American Review of Respiratory Disease. 1972; Vol. 105(issue 6):883-90. [PubMed: 5032708]

Tattersfield 2006. Tattersfield AE. Current issues with beta2-adrenoceptor agonists: historical background. Clinical Reviews in Allergy \& Immunology. 2006; Vol. 31(issue 2-3):107-18. [PubMed: 17085787]

Tonnel 2001. Tonnel AB, Gosset P, Tillie-Leblond I. Characteristics of the inflammatory response in bronchial lavage fluids from patients with status asthmaticus. International Archives of Allergy and Immunology. 2001; Vol. 124(issue 1-3):267-71. [PubMed: 11306987]

Van der Woude 2001. Van der Woude HJ, Winter TH, Aalbers R. Decreased bronchodilating effect of salbutamol in relieving methacholine induced moderate to severe bronchoconstriction during high dose treatment with long acting $\beta_{2}$ agonists. Thorax. 2001; Vol. 56(issue 7):529-35. [PubMed: 11413351]

Van Noord 1996. Van Noord JA, Smeets JJ, Raaijmakers JAM, Bommer AM, Maesen FPV. Salmeterol versus formoterol in patients with moderately severe asthma: onset and duration of action. European Respiratory Journal. 1996; 9(8):1684-8. [PubMed: 8866595]

Vignola 1993. Vignola AM, Campbell AM, Chanez P, Bousquet J, Paullacoste P, Michel FB, et al. HLA-DR and ICAM-1 expression on bronchial epithelial-cells in asthma and chronic bronchitis. American Review of Respiratory Disease. 1993; Vol. 148(issue 3):689-94. [PubMed: 8103654]

Walters 2002. Walters EH, Walters JAE, Gibson PW. Regular treatment with long acting beta agonists versus daily regular treatment with short acting beta agonists in adults and children with stable asthma. Cochrane Database of Systematic Reviews. 2002; (Issue 3) [DOI: 10.1002/14651858.CD003901].

Walters 2007. Walters EH, Gibson PG, Lasserson TJ, Walters JAE. Long-acting beta2-agonists for stable chronic asthma. Cochrane Database of Systematic Reviews. 2007; (Issue 1) [DOI: 10.1002/14651858.CD001385.pub2]. 
Weber 1982. Weber RW, Smith JA, Nelson HS. Aerosolized terbutaline in asthmatics - development of subsensitivity with long-term administration. Journal of Allergy and Clinical Immunology. 1982; Vol. 70(issue 6):417-22. [PubMed: 6815252]

Wiggs 1990. Wiggs BR, Moreno R, Hogg JC, Hilliam C, Pare PD. A model of the mechanics of airway narrowing. Journal of Applied Physiology. 1990; Vol. 69(issue 3):849-60. [PubMed: 2246172]

Wilson 1981. Wilson JD, Sutherland DC, Thomas AC. Has the change to beta-agonists combined with oral theophylline increased cases of fatal asthma. Lancet. 1981; Vol. 1(issue 8232):1235-7. [PubMed: 6112568]

Wong 1990. Wong CS, Pavord ID, Williams J, Britton JR, Tattersfield AE. Bronchodilator, cardiovascular, and hypokalemic effects of fenoterol, salbutamol, and terbutaline in asthma. Lancet. 1990; Vol. 336(issue 8728):1396-9. [PubMed: 1978872]

Woodruff 2004. Woodruff PG, Dolganov GM, Ferrando RE, Donnelly S, Hays SR, Solberg OD, et al. Hyperplasia of smooth muscle in mild to moderate asthma without changes in cell size or gene expression. American Journal of Respiratory and Critical Care Medicine. 2004; Vol. 169(issue 9):1001-6. [PubMed: 14726423]

Yates 1996. Yates DH, Kharitonov SA, Barnes PJ. An inhaled glucocorticoid does not prevent tolerance to the bronchoprotective effect of a long-acting inhaled beta(2)-agonist. American Journal of Respiratory and Critical Care Medicine. 1996; Vol. 154(issue 6):1603-7. [PubMed: 8970342]

* Indicates the major publication for the study 


\section{PLAIN LANGUAGE SUMMARY}

\section{Does daily treatment with salmeterol result in more serious adverse events compared with placebo or a salbutamol?}

Asthma is a common condition that affects the airways - the small tubes that carry air in and out of the lungs. When a person with asthma comes into contact with an irritant (an asthma trigger), the muscles around the walls of the airways tighten, the airways become narrower, and the lining of the airways becomes inflamed and starts to swell. This leads to the symptoms of asthma - wheezing, coughing and difficulty in breathing. They can lead to an asthma attack or exacerbation. People can have underlying inflammation in their lungs and sticky mucus or phlegm may build up, which can further narrow the airways. There is no cure for asthma; however there are medications that allow most people to control their asthma so they can get on with daily life.

Long-acting beta ${ }_{2}$-agonists, such as salmeterol, work by reversing the narrowing of the airways that occurs during an asthma attack. These drugs - taken by inhaler - are known to improve lung function, symptoms, quality of life and reduce the number of asthma attacks. However, there are concerns about the safety of long-acting beta 2 -agonists, particularly in people who are not taking inhaled corticosteroids to control the underlying inflammation. We did this review to take a closer look at the safety of people taking salmeterol daily compared to people on placebo or the short acting beta 2 -agonist salbutamol.

There was no statistically significant difference in the number of people who died during treatment with salmeterol compared with placebo or salbutamol. Because so few people die of asthma, huge trials or observational studies are normally required to detect a difference in death rates from asthma. There were more non-fatal serious adverse events in people taking salmeterol compared to those on placebo; for every 188 people treated with salmeterol for 28 weeks, one extra non-fatal event occurred in comparison with placebo. There was no significant differences in serious adverse events in people on salmeterol compared to regular salbutamol.

In order to obtain individual patient data on asthma deaths, we looked separately at mortality in two large trials on over 51,000 patients who were not taking inhaled corticosteroids, and found that there was an increase in the number of asthma-related deaths among people on salmeterol.

We conclude that, for patients whose asthma is not well-controlled on moderate doses of inhaled corticosteroids, additional salmeterol can improve symptoms but this may be at the expense of an increased risk of serious adverse events and asthma related mortality. Salmeterol should not be used as a substitute for inhaled corticosteroids, and adherence with inhaled steroids should be kept under review if separate inhalers are used.

Salmeterol should not be taken by people who are not taking regular inhaled steroids due to the increased risk of asthma-related death. 


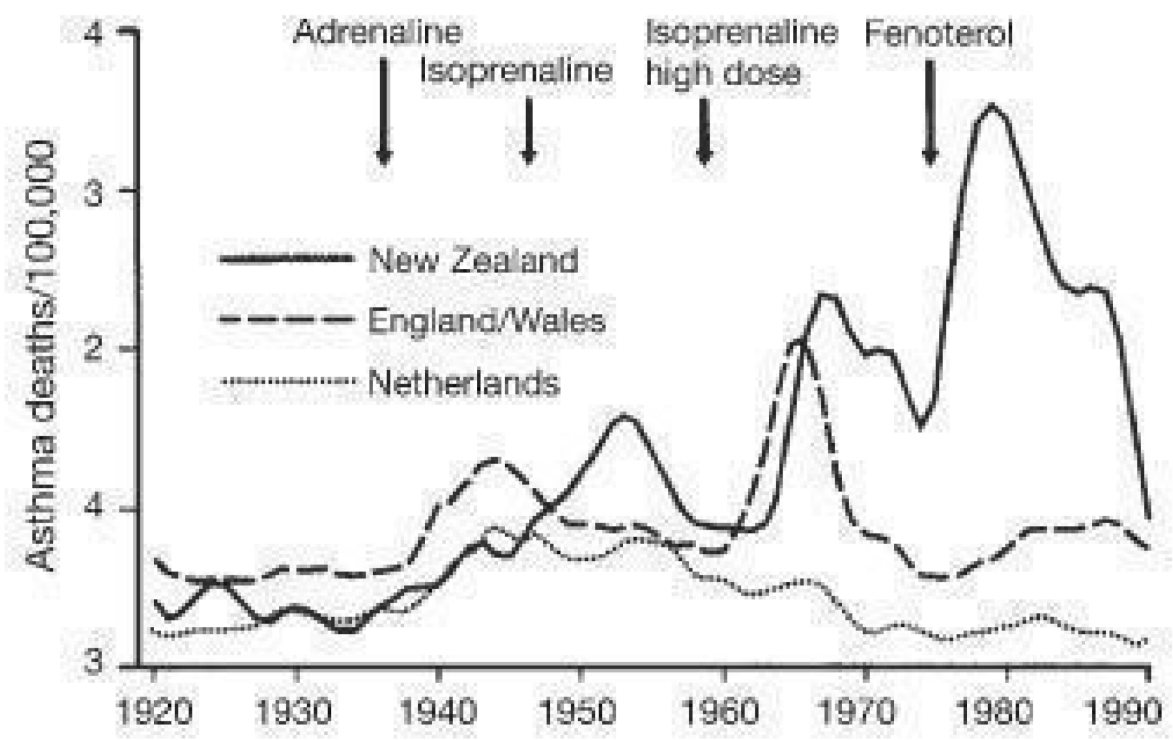

Figure 1. Changes in asthma mortality (5-34 age group) in three countries in relation to the introduction of isoprenaline forte in the UK and New Zealand and of fenoterol in New Zealand. (From Blauw 1995. With permission from the Lancet.) 


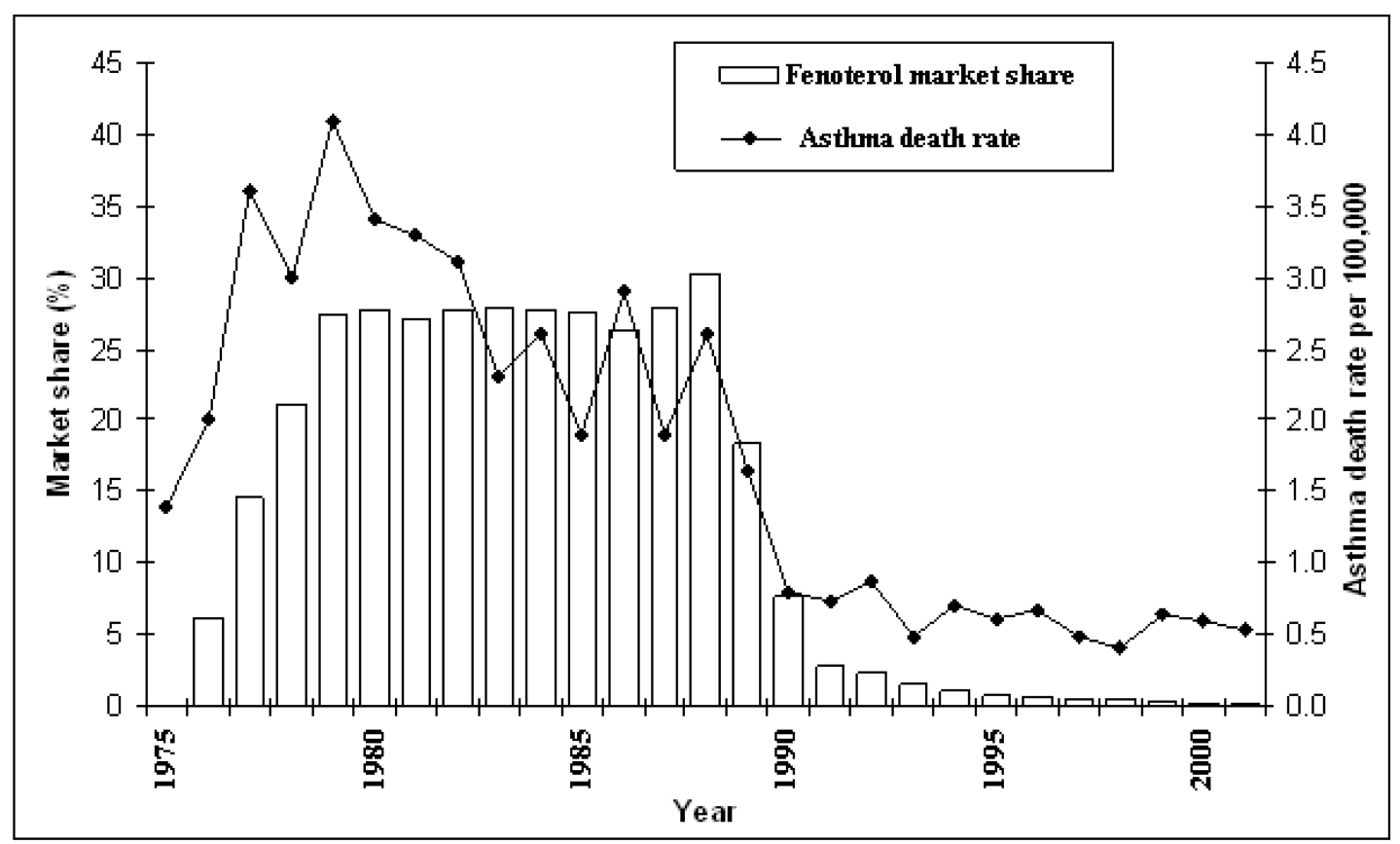

Figure 2. Inhaled fenoterol market share and annual asthma mortality in New Zealand in persons aged 5-34. 


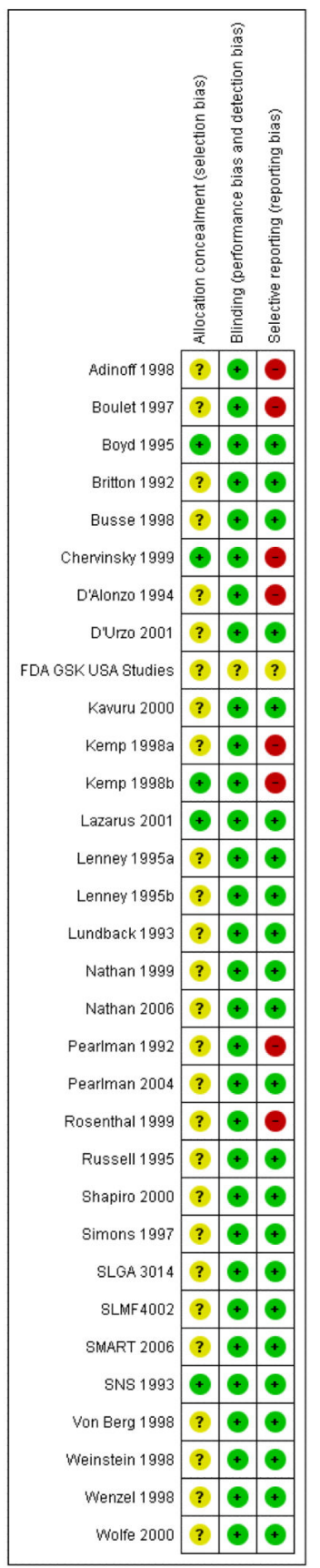

Figure 3. Risk of bias summary: review authors' judgments about each risk of bias item for each included study. 


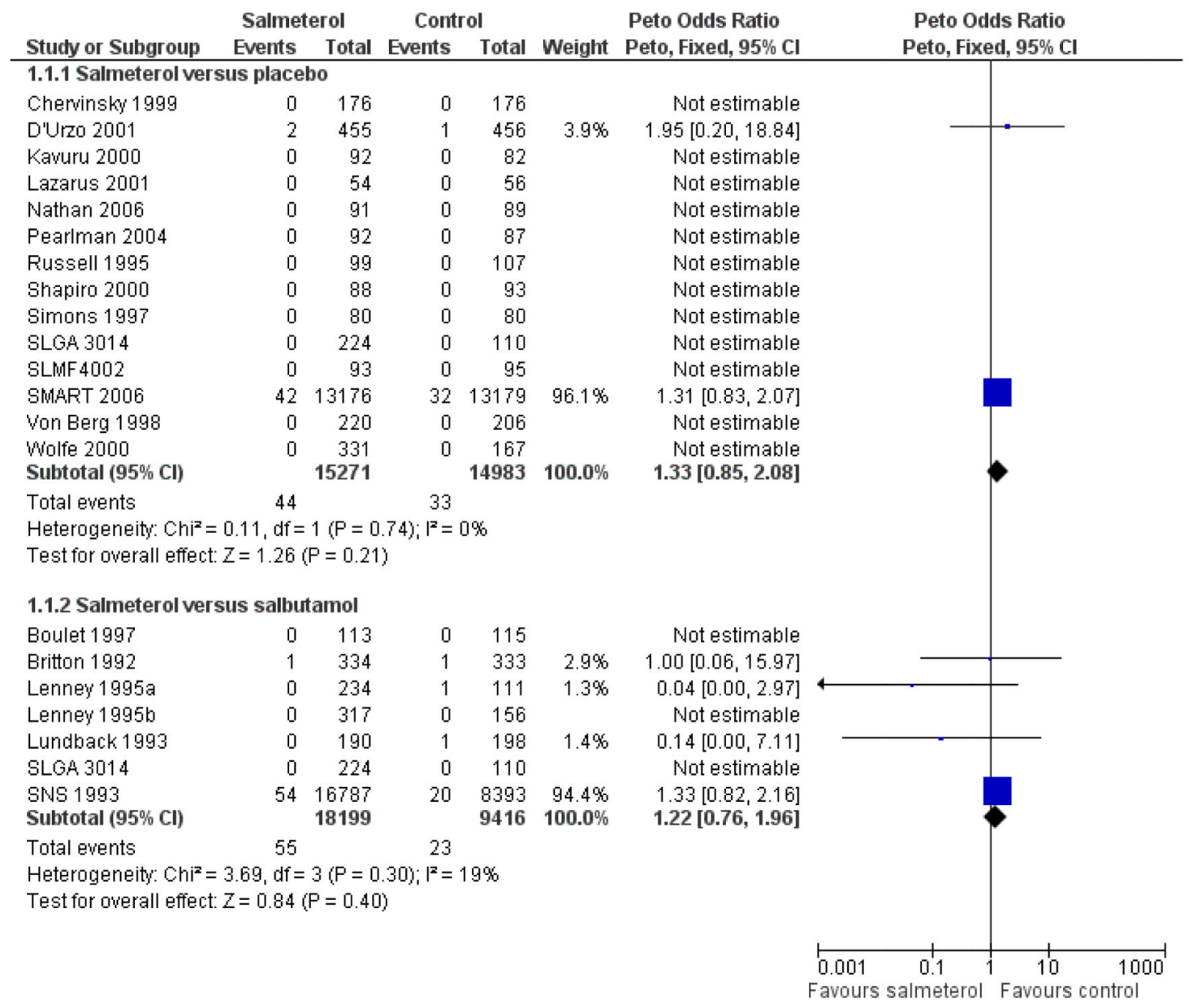

Figure 4. Forest plot of comparison: 1 All-cause Mortality, outcome: 1.1 Overall results. 


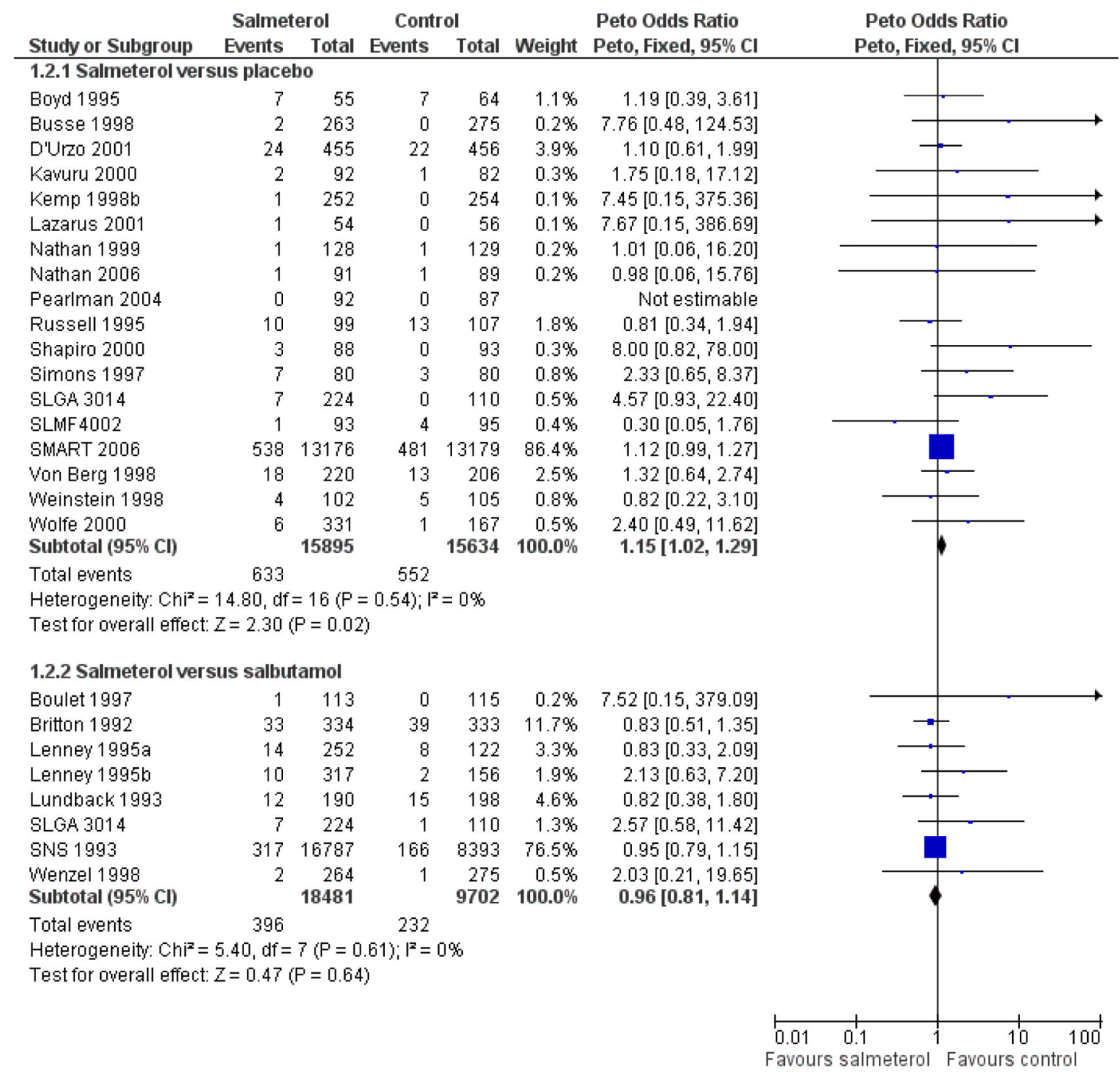

Figure 5. Forest plot of comparison: 1 Regular salmeterol versus placebo or regular salbutamol, outcome: 1.2 Non-fatal serious adverse events (adults and children). 


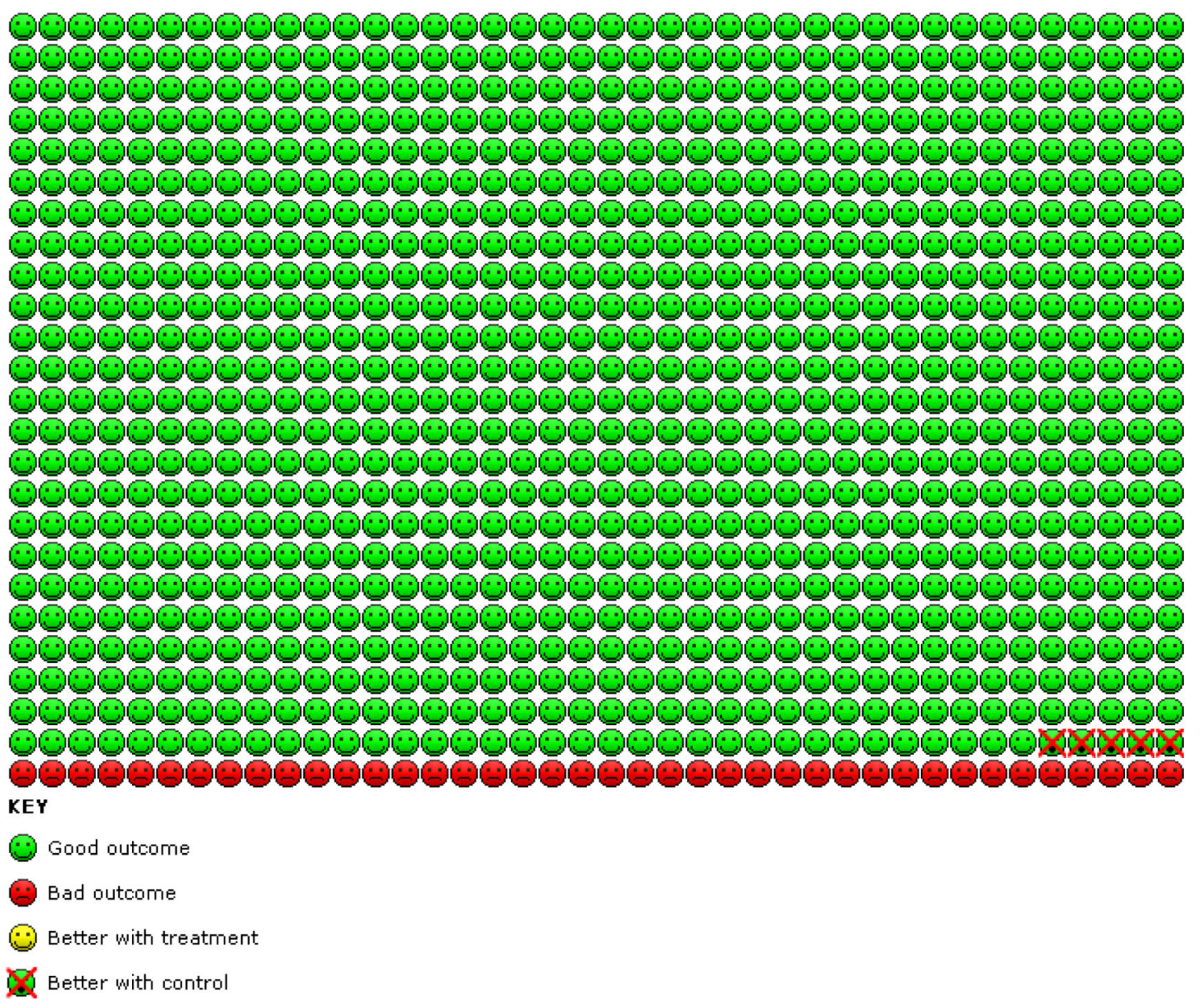

Figure 6. Serious adverse events with salmeterol in comparison to placebo.

For 1000 patients given regular salmeterol for 28 weeks there would be 45 patients who suffer a serious adverse event, in comparison with 40 if all 1000 were given placebo. 


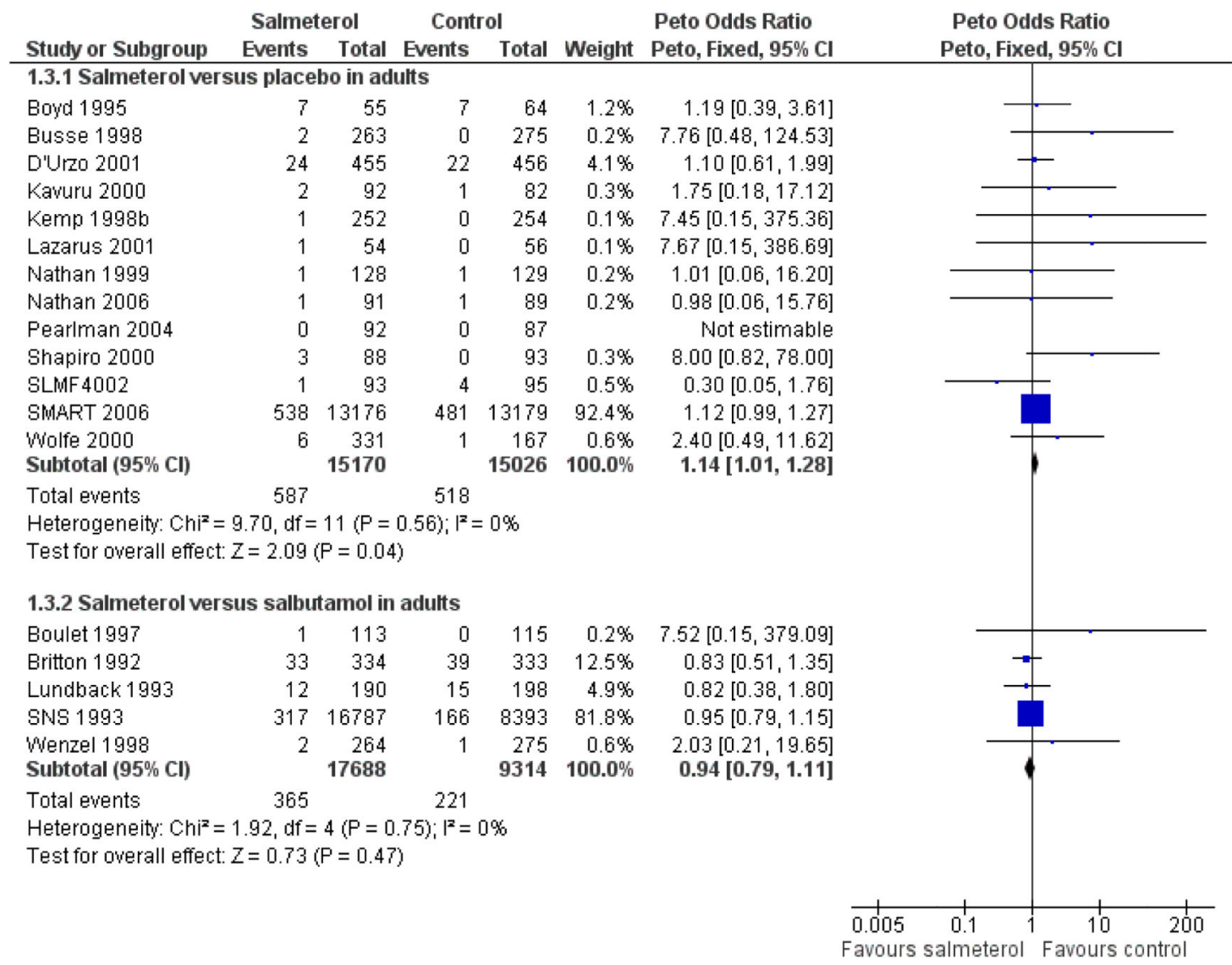

Figure 7. Forest plot of comparison: 1 Regular salmeterol versus placebo or regular salbutamol, outcome: 1.3 Non-fatal serious adverse events in adults. 


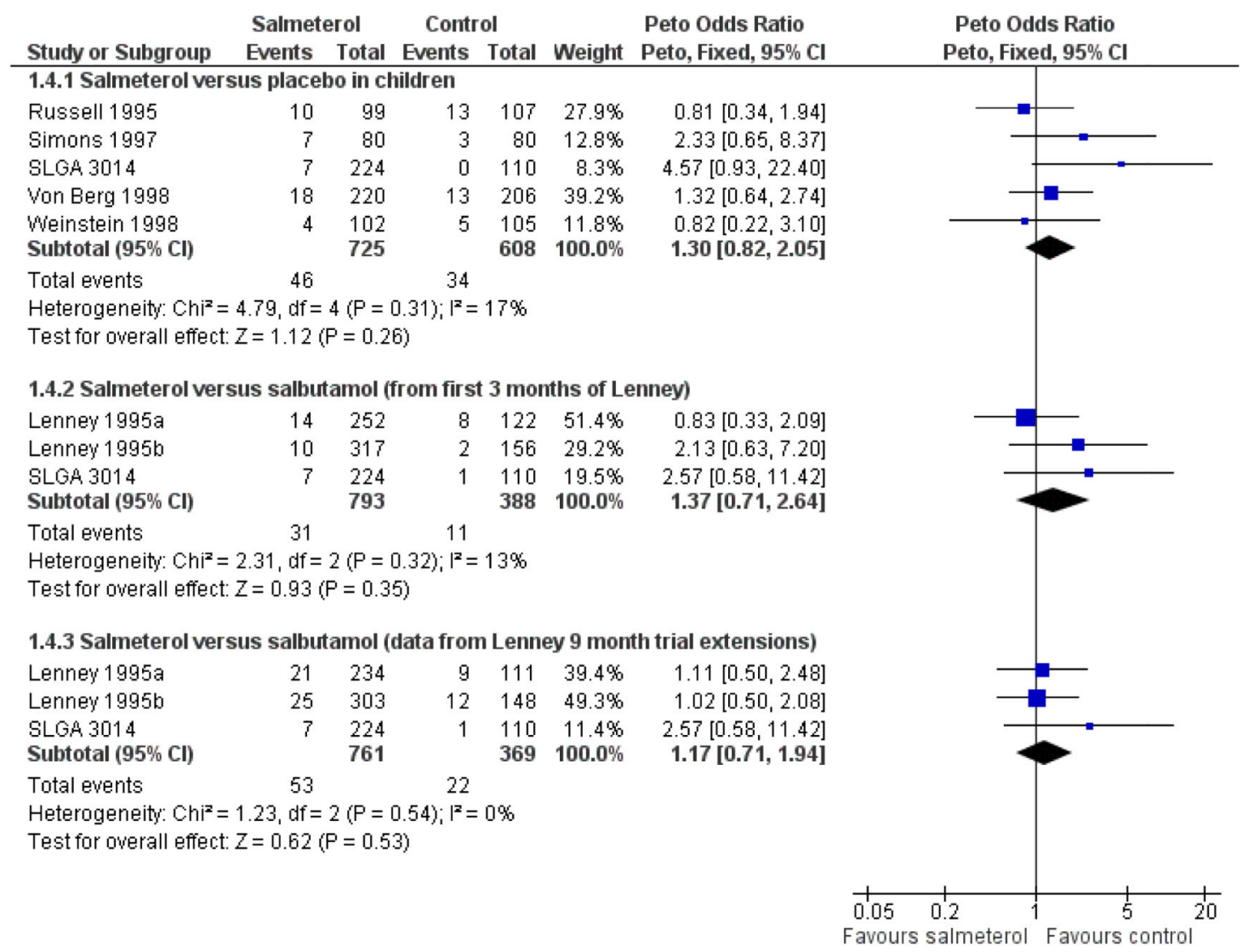

Figure 8. Forest plot of comparison: 1 Regular salmeterol versus placebo or regular salbutamol, outcome: 1.4 Non-fatal serious adverse events in children. 


\section{Table 1}

\section{Baseline use of inhaled corticosteroids}

\begin{tabular}{ll}
\hline Study ID & \% patients \\
Adinoff 1998 & 64 \\
Boulet 1997 & 74 \\
Boyd 1995 & 100 \\
Britton 1992 & 64 \\
Busse 1998 & 67 \\
Chervinsky 1999 & 51 \\
D'Alonso 1994 & 21 \\
D’Urso 2001 & 93 \\
Kavuru 2000 & 26 - Withdrawn \\
Kemp 1998a & 43 \\
Kemp 1998b & 100 \\
Lazarus 2001 & 53 - Withdrawn \\
Lenney 1995 & 57 \\
Lundback 1993 & 31 \\
Nathan 1999 & 57 - Withdrawn \\
Nathan 2006 & 100 - Withdrawn \\
Pearlman 1992 & 25 \\
Pearlman 2004 & 37 - Withdrawn \\
Rosenthal 1999 & 0 \\
Russell 1995 & 100 \\
Shapiro 2000 & 100 - Withdrawn \\
Simons 1997 & 0 \\
SLGA 3014 & 50 \\
SLMF4002 & 100 \\
SMART 2006 & 47 \\
SNS 1993 & 69 \\
Von Berg 1998 & 52 \\
Weinstein 1998 & 57 \\
Wenzel 1998 & 47 \\
Wolfe 2000 & 33 \\
\hline
\end{tabular}




\section{Table 2}

\section{Intrinsic efficacy of beta-agonists}

\begin{tabular}{ll}
\hline Drug & Intrinsic efficacy (\%) \\
Isoprenaline, adrenaline & 100 \\
Fenoterol & 42 \\
Formoterol & 20 \\
Salbutamol & 4.9 \\
Salmeterol & $<2$ \\
\hline
\end{tabular}

Adapted from Hanania 2002. The authors acknowledge that it is difficult to determine the intrinsic efficacy of salmeterol given its high lipophilicity 